\title{
March 2001 Working Group Meeting on Heavy Vehicle Aerodynamic Drag: Presentations and Summary of Comments and Conclusions
}

R. McCallen, R. Greenman, D. Flowers, T. Dunn, J. Owens, G. Laskowski, LLNL; F. Browand A. Knight, and M. Hammache, University of Southern California; $A$. Leoard and M. Rubel, California Institute of Technology; K. Salari and W. Rutledge, Sandia National Laboratories; J. Ross, D. Satran, J.T. Heineck, S. Walker, D. Driver, B. Storms, NASA Ames Research Center

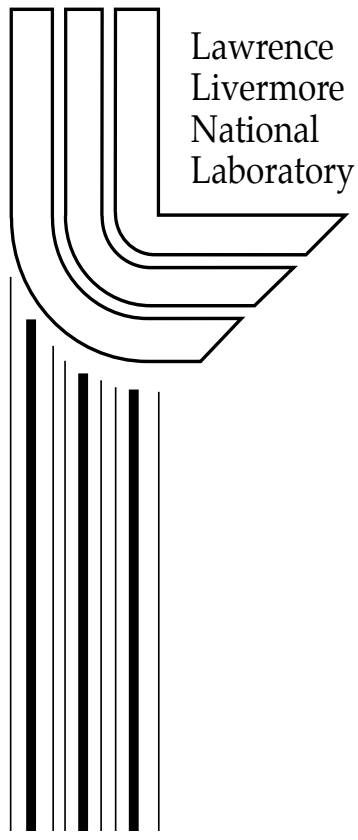

\section{May 14, 2001}




\section{DISCLAIMER}

This document was prepared as an account of work sponsored by an agency of the United States Government. Neither the United States Government nor the University of California nor any of their employees, makes any warranty, express or implied, or assumes any legal liability or responsibility for the accuracy, completeness, or usefulness of any information, apparatus, product, or process disclosed, or represents that its use would not infringe privately owned rights. Reference herein to any specific commercial product, process, or service by trade name, trademark, manufacturer, or otherwise, does not necessarily constitute or imply its endorsement, recommendation, or favoring by the United States Government or the University of California. The views and opinions of authors expressed herein do not necessarily state or reflect those of the United States Government or the University of California, and shall not be used for advertising or product endorsement purposes.

This is a preprint of a paper intended for publication in a journal or proceedings. Since changes may be made before publication, this preprint is made available with the understanding that it will not be cited or reproduced without the permission of the author.

This report has been reproduced directly from the best available copy.

Available electronically at http://www.doc.gov/bridge

Available for a processing fee to U.S. Department of Energy

And its contractors in paper from

U.S. Department of Energy

Office of Scientific and Technical Information

P.O. Box 62

Oak Ridge, TN 37831-0062

Telephone: (865) 576-8401

Facsimile: (865) 576-5728

E-mail: reports@adonis.osti.gov

Available for the sale to the public from

U.S. Department of Commerce

National Technical Information Service

5285 Port Royal Road

Springfield, VA 22161

Telephone: (800) 553-6847

Facsimile: (703) 605-6900

E-mail: orders@ntis.fedworld.gov

Online ordering: http:/ / www.ntis.gov/ordering.htm

\section{OR}

Lawrence Livermore National Laboratory

Technical Information Department's Digital Library

http: / / www.llnl.gov/tid/Library.html 


\title{
March 2001 \\ Working Group Meeting on \\ Heavy Vehicle Aerodynamic Drag: \\ Presentations and Summary of Comments and \\ Conclusions
}

\author{
Jointly written by \\ Lawrence Livermore National Laboratory \\ Sandia National Laboratories \\ University of Southern California \\ California Institute of Technology \\ NASA Ames Research Center
}

\section{Introduction}

A Working Group Meeting on Heavy Vehicle Aerodynamic Drag was held at Lawrence Livermore National Laboratory on March 28 and 29, 2001. The purpose of the meeting was to present and discuss technical details on the experimental and computational work in progress and future project plans. Due to the large participation from industry and other research organizations, a large portion of the meeting (all of the first day and part of the second day) was devoted to the presentation and discussion of industry's perspective and work being done by other organizations on the demonstration of commercial software and the demonstration of a drag reduction device. This report contains the technical presentations (viewgraphs) delivered at the Meeting, briefly summarizes the comments and conclusions, and outlines the future action items.

Industrial representatives from International Truck and Engine Corporation, PACCAR, and Freightliner participated in discussions and presented an industrial perspective. Representatives from Argonne National Laboratory presented an overview of computational results for heavy vehicles given to them by Adapco Corporation and a representative from Dynacs Corporation presented his plans for demonstration of a variety of commercial and NASA software packages. A representative of Georgia Technology Research Institute (GTRI) presented their experimental results and future plans for a drag reduction device developed by GTRI. The meeting introductory remarks included an overview of the Laboratory provided by a representative of LLNL's Energy Directorate.

Representatives from the Department of Energy (DOE) Office of Transportation Technology Office of Heavy Vehicle Technology (OHVT), Lawrence Livermore National 
Laboratory (LLNL), Sandia National Laboratories (SNL), NASA Ames, University of Southern California's (USC), and California Institute of Technology (Caltech) gave presentations on the DOE Truck Aerodynamics Project and participated in discussions. The second day of the meeting included a review of experimental results and plans by the USC and NASA Ames, the computational results from LLNL and SNL for the integrated tractor-trailer benchmark geometry called the Ground Transportation System (GTS) Model, and turbulence model development and benchmark simulation for rounded cube shapes representative of a tractor and trailer being investigated by the Caltech. NASA Ames also presented information on the new geometry called the Generic Conventional Model (GCM) currently being evaluated in the 7x10 wind tunnel at NASA. Much of the second day discussion involved wind tunnel testing plans, analysis of existing experimental data, simulation results, and needed modeling improvements. One focus topic was the issue of careful verification and validation processes for software evaluation.

\section{Summary of Major Issues}

There were 3 major issues raised at the meeting.

1. A major topic of discussion was the process proposed for evaluation of NASA and commercial software. The Dynacs representative has proposed the evaluation of three separate NASA codes and three separate commercial codes for application to the Generic Conventional Model (GCM) by comparison of one or limited grid cases to experimental data. The question was raised whether the software could be evaluated without standard practices of verification and validation (e.g., demonstration of grid and time convergence)?

2. Another active topic of discussion was the DOE full-scale demonstration experiments proposed for the GTRI blowing device. A planning meeting at GTRI was suggested where industry participation will be encouraged.

3. The DOE Team has NEAR-term deliverables for FY01 that will be of value to industry in providing guidance in the use of RANS models. The fact that plans are to complete this effort by October $1^{\text {st }}$ of this year was not made clear, and the Team was criticized for being 'too academic' and having deliverables that were 'too long term'. The Team did emphasize that they wish to present this information to industry and obtain feedback through an exchange of information during site visits and formal documentation. The majority was supportive of this communication approach. 


\section{Introduction, Overview of the Project, and Future Activities}

After Ray Smith of LLNL gave an introduction to the Laboratory, Rose McCallen of LLNL presented the project overview. Some comments from the industry participants included

- Suggestion that an 'intermediary' for collaboration would be helpful,

- Separate meeting to update the multi-year program plan was recommended,

- Request that industry input be considered for wind tunnel model geometries, and

- Comment that industry wants near-term results.

$\bullet$

Sid Diamond of DOE OHVT provided an update of the OHVT projects and budgets and some inspiring thoughts. He encouraged a consortium with industry with a 'modest' cost share from industry. His vision includes the involvement of not only the tractor manufacturers but also the added participation from trailer manufacturers, because of the well-recognized importance of integrating the tractor and trailer for reduction of aerodynamic drag.

The viewgraphs for the project introduction and LLNL overview are attached at the end of this report.

\section{Industrial Perspective}

Sunil Jain of International Truck and Engine Corporation emphasized that reducing drag is their objective and that the $21^{\text {st }}$ Century Truck Initiative goal to reduce the drag by $25 \%$ over 20 years should be feasible with a strong team involving a tractor and trailer group. The wake and gap flow are the critical areas that need improvements for drag reduction. Sunil also provided an overview of the past and current aerodynamic effort at his company. International uses various commercial codes dependent on whether they are modeling internal, external components, or whole body flow. The predicted and measured drag are not always in agreement, but the commercial codes do provide a good match with wind tunnel pressures and good qualitative results. It is important, and often sufficient, to predict the correct changes in drag or correct trends. Typical problems involve 1-2 million zones, but can be up to 6-7 million zones. For underhood flows, changes in temperature need to be predicted to within 5\%. Simple source and sink models for the fan are not adequate and more detailed models are required. The importance of understanding the flow phenomena, determining optimum vehicle shape to minimize drag, and to be able to correlate the computed and experimental drag coefficient was discussed. It was suggested that the DOE Truck Aero Team provide industry with the CFD tools they are using so that they can investigate their use. It was also suggested that the Team establish collaborative relationships with the commercial software industry providing advanced modeling guidance that will enhance those tools now being used by the tractor 
manufacturers. Participation at the November 2001 SAE Truck and Bus Meeting in Chicago was encouraged. Further details are provided on Sunil's viewgraphs.

Dan Schlesinger of Freightliner provided an overview of market situations, overall development activities to reduce fuel consumptions, important issues in the design process to consider, aerodynamic testing, and CFD analysis. It was recognized that improvements in aerodynamic drag have the biggest payoff but the customer is not ready for an integrated tractor-trailer, the cubic capacity of the trailer can't be sacrificed so that a generous leading-edge radii is not acceptable, and bottails have overall length restrictions. It would be advantageous to have aerodynamics influence the design process upfront, but this is difficult if modifications or additions add cost, assembly time, complexity, or negatively impacts styling. It is also difficult to impact the design if the aerodynamic analysis takes too long. Wind tunnel testing can provide adequate changes in drag, but often not the absolute drag. CFD analysis is accomplished using commercial tools for internal flows, small-scale component analysis, and full body flow.

Everett Chu from PACCAR Technical Center provided an overview of his company's vehicle-aerodynamics development program including a description of their HeavyVehicle Manufacturing Divisions, methods used for product performance assurance, the use of CFD tools, wind tunnel experiments, and vehicle/track confirmation tools. PACCAR's Technical Center can provide support to it's other subsidiaries including Kenworth (US), Peterbilt (US), DAF (Netherlands), Foden (UK), and Leyland Trucks (UK). The Technical Center located in Mount Vernon, Washington has a 1.6-mile test track. Their product performance assurance follows a 4-stage development process including benchmarking and target setting, advanced concept, prototype validation, and product confirmation. CFD tools, wind tunnel experiments, and vehicle/track testing are part of this process. Different commercial CFD codes are used for application to underhood or external flows. Wind tunnel scaling issues (e.g., variation in results for different scale models) and several concerns involving the GCM design were mentioned (e.g., wheels too hidden, areas closed and open, cantilever of front end). Everett also provided input on the DOE program including 'helpful tools and techniques' and 'helpful analyses for specific vehicle areas and components' which are attached in the viewgraph section of this report.

\section{Demonstration of Commercial Tools}

Ross Sheckler of Dynacs Corporation presented his plans for the evaluation of 3 commercial and 3 NASA software packages. The focus of the discussion was on the proposed method of evaluation, as mentioned above, which is a 'best effort' approach. It was suggested that Ross document the criteria and evaluation process for review by the DOE Aero (SWAT) Team, and that both work towards some agreement in the approach. The results would then be submitted to DOE and industry for their review. Another topic was the use of vortex generators on the GCM during the wind tunnel experiments. 
PACCAR representative, Everett $\mathrm{Chu}$, mentioned that the vortex generators have been tested and that it is likely we will not see the results we are looking for. There was also discussion that our testing of the devices may be advertised as an endorsement for their use. Further details are provided in the attached viewgraphs.

Tanju Sofu of ANL presented some calculations performed by the software company Adapco using their code STARCD. Typical calculations involve roughly 10 million zones for simulation of automobile aerodynamics and 20-30 million zones for tractor-trailer simulations. Tabulated results are provided in the attached viewgraphs.

\section{Aerodynamic Devices}

Bob Englar of GTRI presented the results of wind tunnel experiments using a modified GTS with wheels, a gap between the tractor and trailer, with and without squared or rounded trailer leading and trailing edges, and tractor-trailer height mismatch. It was found that including the wheels on the GTS increased the drag. Rounding the leading edge of the trailer reduced the tractor-trailer height-mismatch imposed drag. Rounding the trailer rear corners is also helpful. Bob also presented the results of using a blowing device. Details can be found in the attached draft SAE paper to be presented at the 2001 SAE Government/Industry Meeting. Discussions on these results focused on plans for an upcoming demonstration full-scale field test. Questions raised were about the power source, what kind of instrumentation would be included on the field tests, what would be the cost of the tests, what are the ramifications of blowing: for example, would blowing have substantial drive-by noise, would icing be a problem, and what were the system maintenance issues. A meeting is scheduled in May in Atlanta to finalize the plans for the field tests and to further address some of these questions.

\section{DOE Truck Aerodynamic ('SWAT') Team's Presentations}

\section{NASA's Current Tests on GCM in 7-ft $x$ 10-ft Wind Tunnel Experiments and Plans for 12-ft Wind Tunnel Experiments}

Jim Ross of NASA Ames presented the final report on the GTS 7-ft x10-ft experiments, some preliminary results on the 7-ft x10-ft experiments on the GCM which were started on March $19^{\text {th }}$, and the planning and preparation underway for the 12-ft pressure wind tunnel experiments with the GCM. Some of the parameters to be investigated with the GCM are Continuum Dynamics, Inc boattail plates, tractor trailing edge side extenders at two different lengths, vortex generators and under-slung cargo. Instrumentation will include 256 pressure taps, particle image velocimetry (PIV) in the tractor-trailer gap and at the trailer base, oil film interferometry (OIF) for measuring skin friction, unsteady pressure transducers at rear of side extenders, inside the gap surface and the back of the trailer, and independent measurement of tractor and trailer drag. The preliminary results with the GCM indicate a 'break' in the drag curve at $10^{\circ}$-yaw, which was not seen with 
the GTS model. One hypothesis is that this characteristic is present because of the tractor-trailer gap flow. Jim also gave a reminder that the fairings on the bottom posts are not connected to the balance, so that one should not integrate over the fairing in calculating drag coefficients. Details of the NASA test plans are provided in the attached viewgraphs.

\section{USC's Wind Tunnel Tests and a Look at an Aero Device}

Fred Browand, Mustapha Hammache, and Albion Knight of USC gave an overview of their preparation work on the United Engineering Foundation Conference, a detailed presentation of their gap flow analysis, and a report on their progress in the development of a base drag reduction device. Fred has put together an advisory committee of outstanding internationally known researchers and industrial representatives from around the world for the United Engineering Foundation Conference on Aerodynamics of Trucks, Busses and Railcars planned for Fall of 2002. Mustapha presented his detailed analysis of the force coefficients as a function of gap and yaw angle. His preliminary conclusions are that at large yaw angles, there is a 'critical' gap where the tractor drag coefficient more than doubles with minimal effect on the trailer drag coefficient, unless the leading edge vertical sides of 'the trailer are rounded, and then trailer drag is also influenced by a 'critical' gap. Mustapha has also developed a novel approach for characterizing the gap flow by dividing the gap into two equal regions and comparing their 'gap outflow' velocities on a 'state space' plot using the PIV data. This method allows for evaluation of the gap flow in the various 'states' by use of conditional sampling based upon the location in the state space. With this type of guidance, industry can make intelligent technical (and economic) decisions on the use of aerodynamic devices for tractor-trailer modifications in the gap region. It was also mentioned that this information would be valuable to the manufacturers and installers of trailer refrigeration systems, who are concerned about air circulation in the gap where the refrigeration system is mounted on the trailer. Details of this described analysis can be found in the attached viewgraph presentation. Albion Knight gave a progress report on his plans for the development and testing of an oscillation device to control the trailer wake flow. The device is intended to alter the turbulent structure of the wake, resulting in a drag reduction. Details of the data acquisition and controls setup for testing the device and a preliminary experimental plan are provided in the attached viewgraphs.

\section{RANS and DES Computations at SNL}

Kambiz Salari of SNL gave an overview of the DOE Accelerated Strategic Computing Initiative (ASCI) and how it provides computer resources that can be utilized for this truck aerodynamics project. Kambiz also presented related issues of code verification and validation $(\mathrm{V} \& \mathrm{~V})$. Progress and status was reported on the Reynolds-averaged Navier Stokes (RANS) computational effort with the GTS geometry with some general findings of the predictive capability of RANS for simulation of a tractor-trailer configuration. 
Information on the development and implementation of a detached-eddy simulation (DES) for application to the GTS model was also presented. (DES is a new turbulence modeling approach where RANS is used in wall regions and LES is used away from walls for reduced grid resolution requirements near walls.) Short-term deliverables for this fiscal year FY01 include careful evaluation of the application of RANS for modeling tractortrailer drag and the transfer of this information to industry as guidance in their simulation process. SNL is investigating 4 different RANS models. The RANS calculations compare well with experiment except for the calculated pressure at the edges of the trailer base. It was recognized that accurate computation of the pressure gradient at the trailing edges of the trailer are essential for correctly determining the vehicle drag. The possible need for edge effect corrections by averaging the pressures for the perpendicular element segments at the edge was mentioned. Unfortunately, SNL has uncovered a 'bug' in their 'block patching' of computational grids. Even though it is believed that the above conclusions will not change, SNL has eliminated this problem and is currently rerunning the simulations to be sure there are no changes in their results. All scheduled tasks should still be completed on time, at the end of FY01. Details of the computations and analysis are provided in the attached viewgraphs.

\section{Simulations using Vortex Methods: A Gridless Technique}

Tony Leonard and Mike Rubel of Caltech gave an update on their progress in the effort to simulate a tractor-trailer configuration using a 'grid-free' (except for 2D grid on body surface) Vortex Method with improved, fast, parallelized, adaptive techniques. Current activities at Caltech include: incorporating bodies with arbitrary complexity, obtaining higher Reynolds numbers computations, and developing and analyzing subgrid models for large-eddy simulation. Using some demonstration calculations, Tony showed the need for 'vortex resolution' to capture the flow in the near wall region at turning edges around the tractor. Mike Rubel of Caltech provided an overview of his development work on a numeric time integration by 'dead reckoning'. This tool is needed to accelerate the vortex method for application to heavy vehicles. Details and results of computations with the vortex method code and on the turbulence modeling approach are in the attached viewgraphs.

\section{LES/DES Incompressible Flow Computations/Analysis at LLNL}

Rose McCallen, Tim Dunn, Roxana Greenman, and Jerry Owens of LLNL reported on the progress and status of their simulation effort. Rose described the near-term deliverables planned for this fiscal year, which include some general conclusions about the effectiveness of LES. Specifically, plans are to generate LES results of the GTS at zero yaw for direct comparison to the experimental data and the RANS simulations performed by SNL. This complimentary work should be of interest to industry, because it will supply them with some near term guidance on the adequacy of various turbulence models for application to predicting the base drag on a tractor-trailer. The approach and 
development challenges were presented by Tim, recent validation results were presented by Roxana, and Jerry demonstrated a new graphics capability that provides movie generation with transparent contours. The code solution speed has in some cases increased by an order of magnitude with more effective use of a Finite Element Interface (FEI) and the introduction of stabilization and solver acceleration schemes. The V\&V results for a flat plate, channel flow, and a sphere show good comparison with experiment. A simulation of the 7x10 NASA wind tunnel indicates promising projections of scaling and timing for LES simulations with the vehicle and tunnel. It was also demonstrated how with LES, stretched grids and adjustable parameters can be manipulated to produce widely different results. Thus, it is possible to 'tweek' parameters to match data, which is not a recommended approach. The LLNL team is struggling with a recent manpower shortage, but every attempt is being made so that scheduled tasks will be completed on time. See attached viewgraphs for details on the models.

\section{Action Items}

The follow-on action items with the individuals responsible for the tasks are as follows:

Unified Engineering Foundation Conference

- $\quad$ Set date and location (R. McCallen)

- Include more international representatives on advisory committee and finalize (F. Browand)

- $\quad$ Send letters requesting support (R. McCallen)

Generate draft document of code evaluation criteria and distribute to SWAT Team for review and then present to DOE and industry for comment (R. Sheckler)

Review and comment on R. Sheckler's evaluation criteria (all)

Attend GTRI meeting in May (Rose, Mustapha)

Generate new SWAT Team budget estimate for FY02 before next meeting (R.

McCallen)

Generate and distribute meeting report with viewgraphs (R. McCallen)

Establish location and schedule next working group meeting (R. McCallen) 
Truck Aero Team Meeting

LLNL, Livermore, CA

March 28-29, 2001

\begin{tabular}{|c|c|c|}
\hline Attendee & Organization & e-mail address and phone \\
\hline Ron Bradley & ORNL & \\
\hline Fred Browand & USC & browand@spock.usc.edu, 213-740-5359 \\
\hline Everett Chu & PACCAR & echu@paccar.com, 360-757-5270 \\
\hline Sid Diamond & DOE & sid.diamond@ee.doe.gov, 202-586-8032 \\
\hline Tim Dunn & LLNL & dunn13@llnl.gov, 925-422-5258 \\
\hline Bob Englar & GTRI & bob.englar@grti.gatech.edu, 770-528-3222 \\
\hline Roxana Greenman & LLNL & greenman2@llnl.gov, 925-424-2501 \\
\hline Mustapha Hammache & USC & hammache@usc.edu, 213-740-5377 \\
\hline Sunil Jain & International & $\begin{array}{l}\text { Sunil.Jain@nav-international.com, 219- } \\
428-3783\end{array}$ \\
\hline Albion Knight & USC & aknight@usc.edu, 213-740-5320 \\
\hline Esther Ku & DOE & esther.ku@ee.doe.gov, 202-586-8179 \\
\hline Tony Leonard & Caltech & tony@galcit.caltech.edu, 626-395-4465 \\
\hline Rose McCallen & LLNL & mccallen1@llnl.gov, 925-423-0958 \\
\hline \multicolumn{2}{|c|}{ Mary McWherter-Payne SNL } & mapayne@sandia.gov, 505-844-8500 \\
\hline Jerry Owens & LLNL & jlowens@llnl.gov, 925-422-1646 \\
\hline Jim Ross & NASA & jcross@mail.arc.nasa.gov, 650-604-6722 \\
\hline Jules Routbort & ANL/DOE & routbort@anl.gov, 630-252-5065 \\
\hline Mike Rubel & Caltech & mrubel@caltech.edu, 626-395-8310 \\
\hline Walt Rutledge & SNL & whrutle@sandia.gov, 505-844-6548 \\
\hline Kambiz Salari & SNL & ksalari@sandia.gov, 505-844-9836 \\
\hline Dan Schlesinger & Freightliner & $\begin{array}{l}\text { Dan Schlesinger@freightliner.com, 503- } \\
745-6975\end{array}$ \\
\hline Ross Sheckler & Dynacs & dynacsny@dynacs.com, 315-626-6800 \\
\hline
\end{tabular}




$\begin{array}{lll}\text { Ray Smith } & \text { LLNL } & \text { smith40@llnl.gov, 925-422-7802 } \\ \text { Tanju Sofu } & \text { ANL } & \text { tsofu@anl.gov, 630-252-9673 } \\ \text { Frank Tokarz } & \text { LLNL } & \text { tokarz1.llnl.gov, 925-423-3459 } \\ \text { Dave Weber } & \text { ANL } & \text { dpweber@anl.gov, 630-252-8175 }\end{array}$




\section{AGENDA}

Heavy Vehicle Aerodynamic Drag: Working Group Meeting Lawrence Livermore National Laboratory

L i v e r m o re, C A

March 28 \& 29, 2001

Building 123, Conf. Room A

\section{Purpose of Meeting}

3. Presentation \& discussion of industry's perspective

4. Presentation $\&$ discussion of technical details of work in progress $\&$ future plans

Wednesday, March 28

\begin{tabular}{|c|c|c|}
\hline \multicolumn{3}{|l|}{ Introduction } \\
\hline $10: 00-10: 30$ & Welcome \& introduction to LLNL & Frank Tokarz \\
\hline $10: 30-11: 00$ & Meeting introduction \& project overview & Rose McCallen \\
\hline $11: 00-11: 45$ & DOE/OHVT update \& budget & Sid Diamond \\
\hline $11: 45-1: 00$ & Working Lunch & \\
\hline \multicolumn{3}{|c|}{ Industry Perspective } \\
\hline $1: 00-1: 30$ & International perspective & Sunil Jain \\
\hline $1: 30-2: 00$ & Freightliner perspective & Dan Schlesinger \\
\hline $2: 00-2: 30$ & Paccar perspective & Everett Chu \\
\hline $2: 30-2: 45$ & Break & \\
\hline $2: 45-3: 45$ & Discussion & \\
\hline \multicolumn{3}{|l|}{ Demonstrations } \\
\hline $3: 45-4: 15$ & Evaluation of commercial tools: plans \& progress & Ross Sheckler \\
\hline $4: 15-4: 45$ & Results with a commercial tool & $\begin{array}{l}\text { Dave Weber, } \\
\text { Tanju Sofu }\end{array}$ \\
\hline $6: 00$ & Dinner at Cattlemen's in Livermore & \\
\hline
\end{tabular}


Thursday, March 29

$$
\text { 7:45 - 8:00 Continental Breakfast }
$$

\section{Summary and Discussion}

$$
\text { 8:00 - 8:30 Introduction, summary, issues from previous day Rose McCallen }
$$

Aerodynamic Devices

$$
\text { 8:30 - 9:30 GTRI project: description \& status Bob Englar }
$$

Work Plans and Progress: Experimental Effort

$$
\begin{array}{lll}
9: 30-10: 30 & \text { NASA data reduction, analysis, documentation, \& test plans Jim Ross } \\
10: 30-10: 45 & \text { Break } & \multicolumn{1}{l}{\begin{array}{ll}
\text { Fred Browand, Mustapha Hammache, } \\
\text { Albion Knight }
\end{array}} \\
10: 45-11: 45 & \text { USC test results, plans, \& aero devices } & \\
11: 45-1: 00 & \text { Working lunch } &
\end{array}
$$

Work Plans and Progress: Computational Effort

$$
\begin{aligned}
& \text { 1:00 - 2:00 SNL RANS computations, analysis \& DES development } \\
& \text { Kambiz Salari, Walt Rutledge, Mary McWherter-Payne } \\
& \text { 2:00 - 3:00 Caltech vortex method development } \& \text { computations } \\
& \text { 3:00 - 4:00 LLNL LES/DES incompressible computations/analysis \& development } \\
& \text { Tim Dunn, Roxana Greenman, Jerry Owens, Rose McCallen } \\
& \text { 4:00 - 5:00 Discussion \& wrap up }
\end{aligned}
$$




\title{
'Working Group Meeting' \\ Consortium for Aerodynamic Drag of Heavy Vehicles
}

\author{
Department of Energy \\ Office of Heavy Vehicle Technology
}

March 28-29, 2001

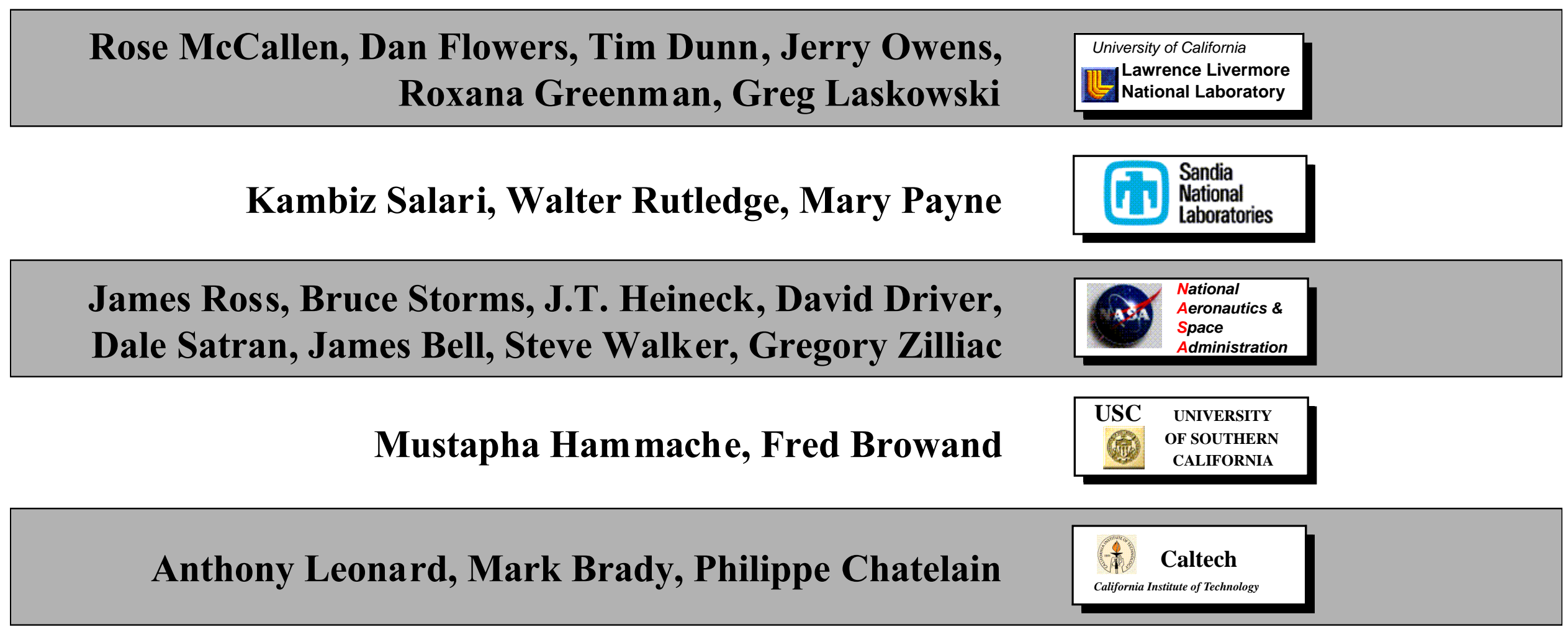

This work was performed under the auspices of the U.S. Department of Energy by University of California Lawrence Livermore National Laboratory under contract No. W-7405-Eng-48. 


\section{DOE wants to reduce fuel consumption by reducing aerodynamic drag.}

At $70 \mathrm{mph}, 65 \%$ of the engine's total energy output is in overcoming aerodynamic drag

Typical Class 8 tractor-trailer

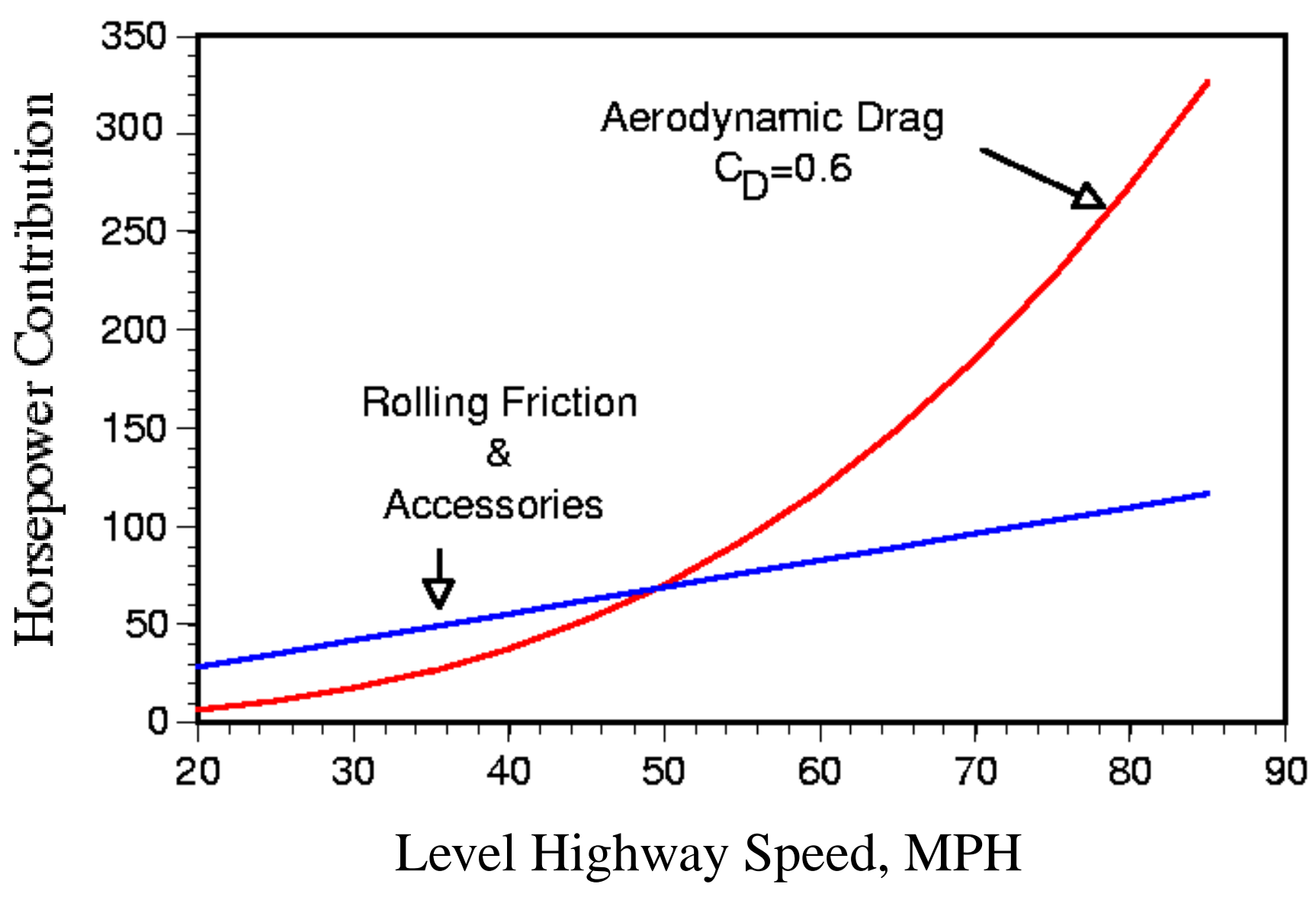




\section{The consortium was formed to provide advanced technology to industry.}

Needed for significant impact on drag

Integrated tractor-trailer

Drag reduction devices

Aerodynamic

Front-end shape trailer-base

components underbody

Improved thermal management (underhood flow)

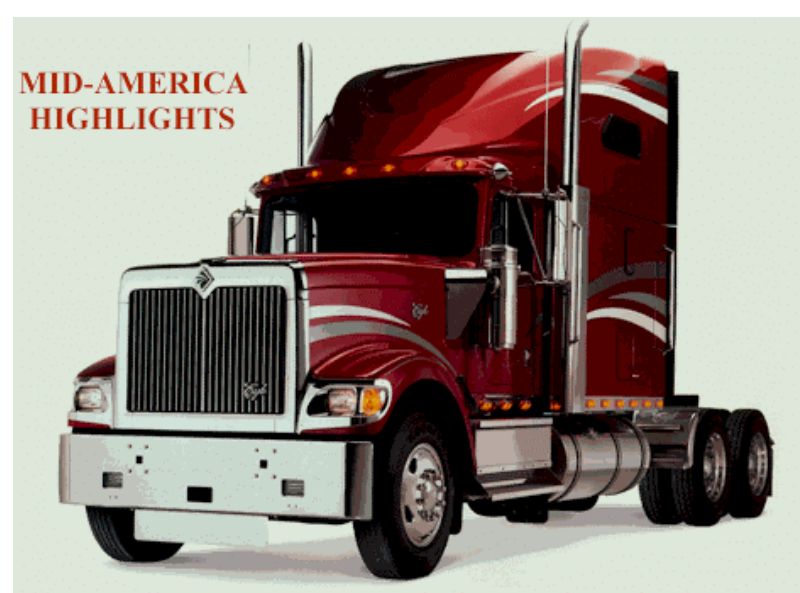

Technologies

Coupling experiments and computations for design guidance

Advanced computational methods and tools

Experimental validation

State-of-the-art experimental techniques

Design and testing 


\section{Approach: A marriage of computations and experiments.}

Perform high quality numerical computations utilizing a variety of modeling schemes

Perform high quality wind tunnel measurements utilizing the most up-todate data acquisition-including DPIV

Perform analyses of results and provide detailed comparisons between numerical results and experimental results (and between various numerical schemes)

Make information available to all interested parties 


\section{The current project focus is on a validated simulation capability and some design effort.}

Computational

Turbulence modeling

Reynolds-averaged Navier-Stokes (RANS) modeling

Large-eddy simulation (LES) modeling

Detached-eddy simulation (DES) modeling

Numerical methods

Finite difference, finite element, and vortex methods

Compressible and incompressible

Experimental validation and phenomena investigation

Ground transportation system (GTS) cab-over model

- full geometry, gap distance, and add-ons

Generic conventional model

Design and testing

Acoustic device for base pressure/wake control

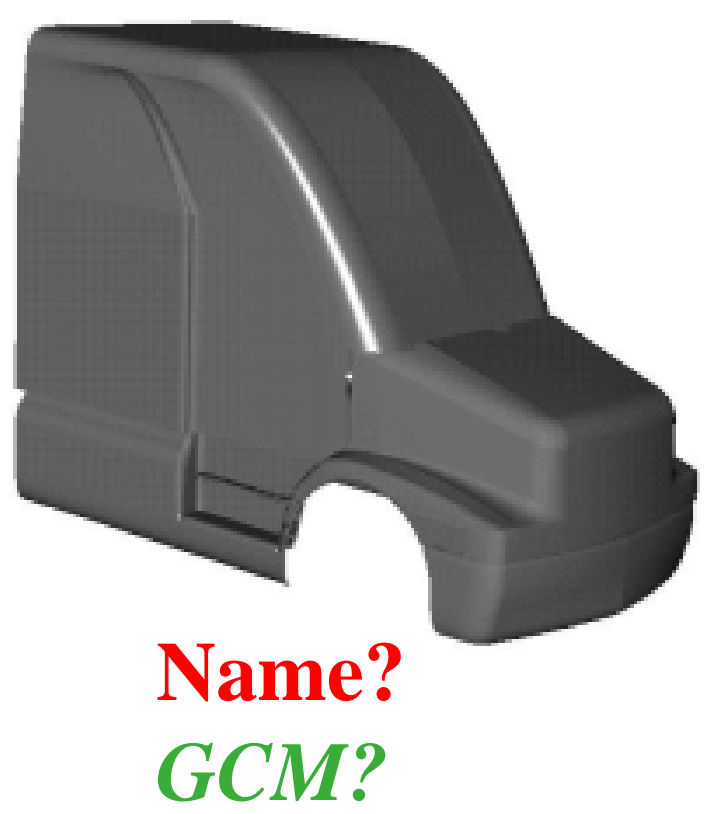




\section{"Vision"}

Applied Research

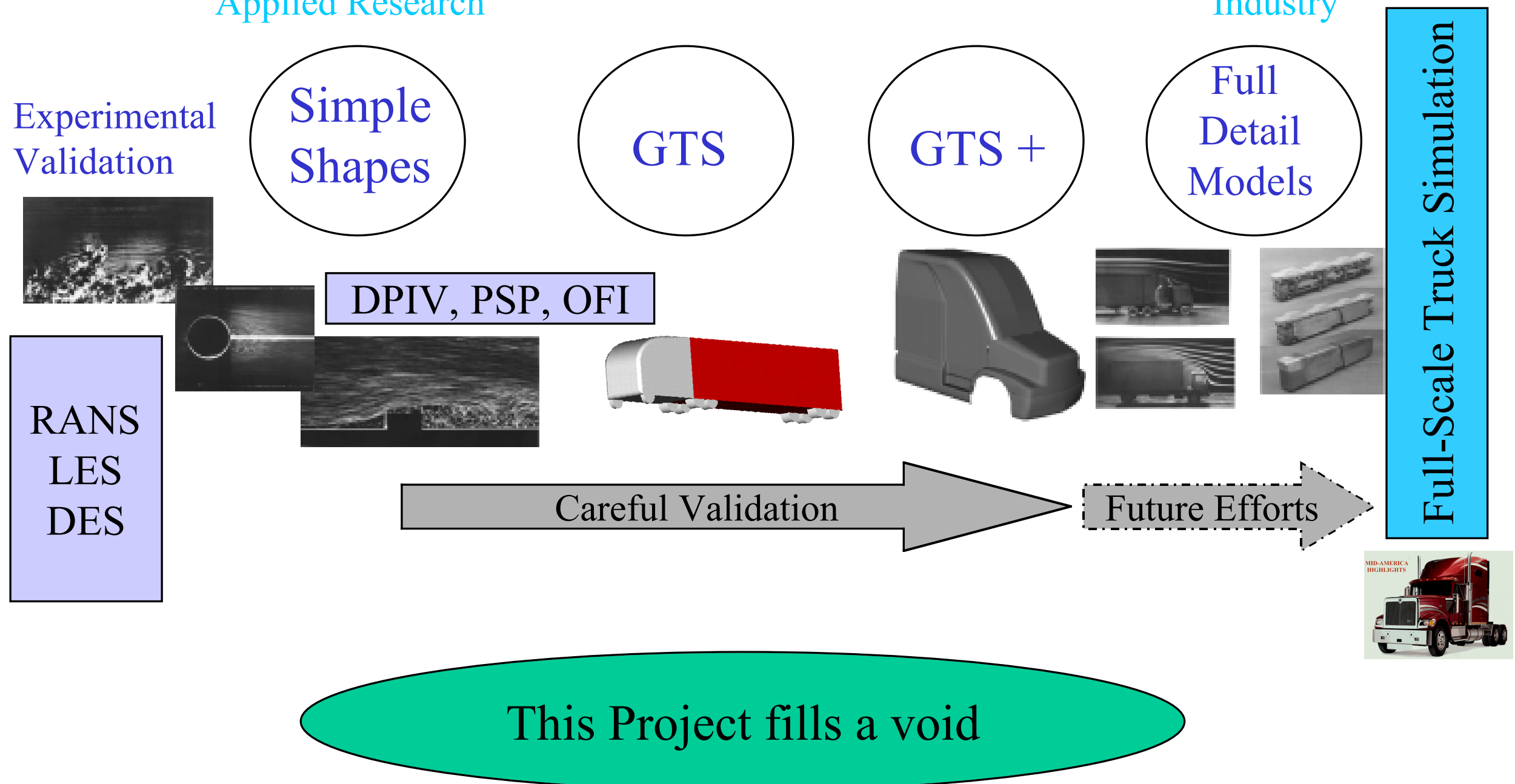




\section{The process will build confidence in CFD and identify areas in need of further development.}

Start simple (numerically and experimentally)

Gain confidence in numerical solutions through established verification and validation processes

Anticipate future revolutionary advances in computer power (push next generation technology)

Demonstrate utility of CFD methods and advanced experimental techniques

Team with Industry and share "lessons learned", methods, and tools.

We are looking for industry participation. 


\section{Accomplishments: The team of experts is established and significant progress has been made.}

- Established multi-lab, multi-university team

- Multi-year program plan in place

- Experiments completed for baseline case (first time 3D, unsteady velocity field measured in a production wind tunnel)

- Understanding of gap flow phenomena

- Preliminary RANS and LES calculations

- Advanced model development in progress

- Continued data base development

- Preliminary design effort

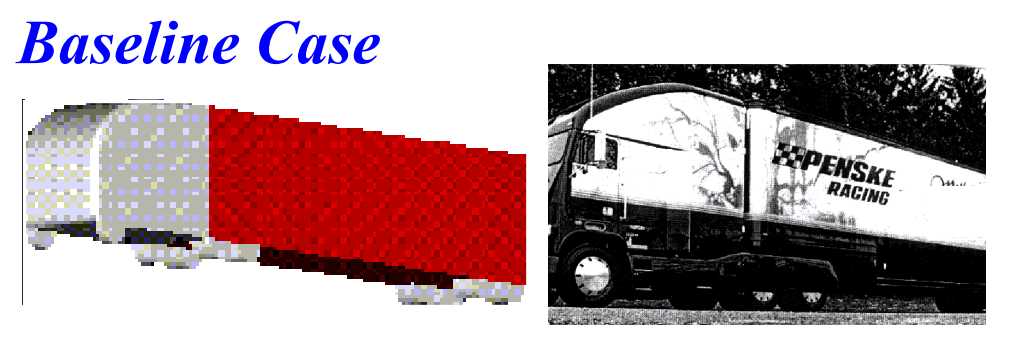

Experiments

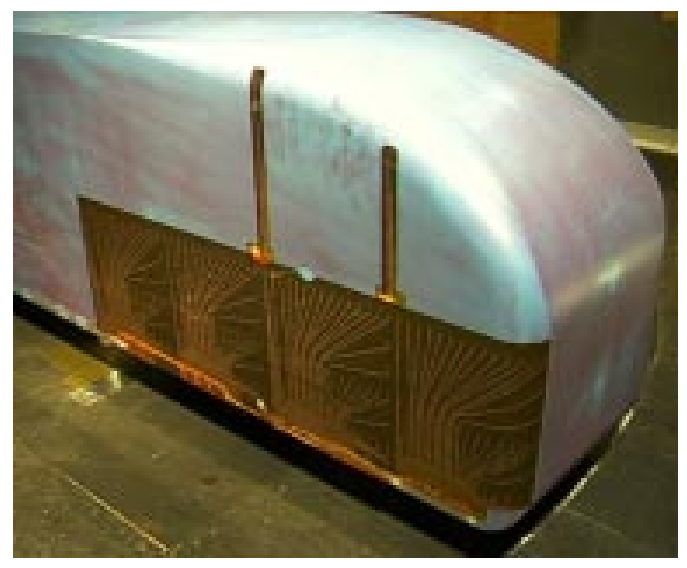

Computations

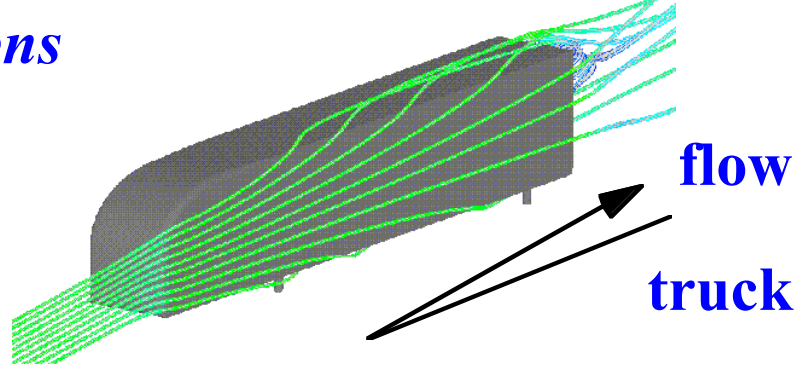




\section{FY01 project tasks in order of priority - submitted with budget}

- $\quad$ - Analysis of existing experimental data with comparison to RANS, LES, and DES computations,

- - Presentation of results to industry through site visits and conference papers,

- $\quad$ - Investigation of various shapes/configurations in the NASA 7-ft x 10-ft wind tunnel, with preparation for FY02 tests in the 12-ft PWT,

- - Development of an acoustic device for reduction of trailer base drag,

- $\quad$ - Continued development of advanced subgrid models for high Re flows,

- $\quad$ - Continued experimental investigation of various shapes and configurations at lower Reynolds number in the USC wind tunnel,

- $\quad$ - Organization of a special Heavy Vehicle Aerodynamics Session at the March 2002 SAE Meeting, and

- $\quad$ - Organization of an Engineering Foundation Conference. 


\section{Break-out by Task}

SNL@Albuquerque

Numerical modeling utilizing Reynolds-Averaged Navier-Stokes and Discrete Edddy $\underline{\text { Simulation }}$

LLNL@Livermore

- Numerical modeling utilizing Large É ddy Simulation

CALTECH@Pasadena

- Numerical modeling utilizing vortex particles as elements

NASA AMES@Moffett Field

High Reynolds number wind tunnel testing including 3D DPIV flow field measurement number, DPIV flow field measurement 


\section{NGV LAUNCH}

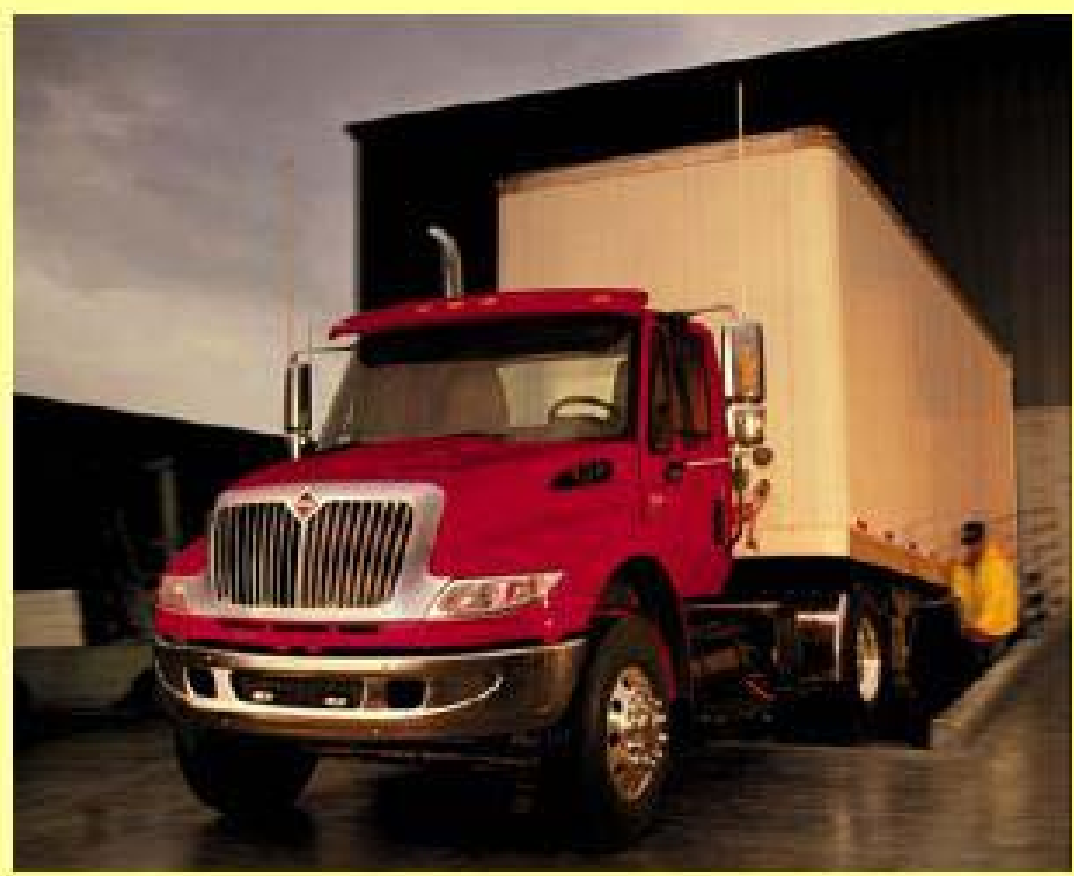

March 28, 2001 


\section{International Perspective}

- Objective:

- Continue Reducing Aerodynamic Drag of Class 8 TractorTrailer System

- Guidelines:

- Each Truck Industry Has Its Own

- 21st Century Truck Initiative

- Reduce Truck Fuel Consumption while Meeting Emission Requirements

- Reduce aerodynamic drag by $25 \%$ over 20 years 


\section{International Perspective}

- Tools used at International

- In-House

- Encouragement from Leadership Team

- Wind Tunnel Tests

- CFD

- Extensive as well as Expensive Knowledge Base of Last 20 Years

- External

- CFD Software Vendors

- International not in Code Development \& CFD Vendors not in Wind Tunnel Test

- Participation in Activities of Various External Agencies

- DOE

- SAE 


\section{Wind Tunnel Test}

- An Important Part of Aerodynamics Group for Last 15 Years

- Consistent Procedure

- Expensive and Time Consuming

- Usage

- Pro-active

- Re-active

- Fundamental Study

- Extensive Wind Tunnel Test Program AISDS - A generic truck

- Complimenting CFD process establishment for the last 2 years

- Extensive Wind Tunnel Test Program AISDS - A generic truck 


\section{AISDS Scope \& Goals}

- Goals

- Better Understand of Truck Shape Interactions

- Develop Correlation between Wind Tunnel Test \& CFD Results

- Approach

- Multi-Phase Parametric Investigations

- Surface Pressure Measurements

- Flow Field Definition 


\section{AISDS Model}

- Generic mathematically-based shapes

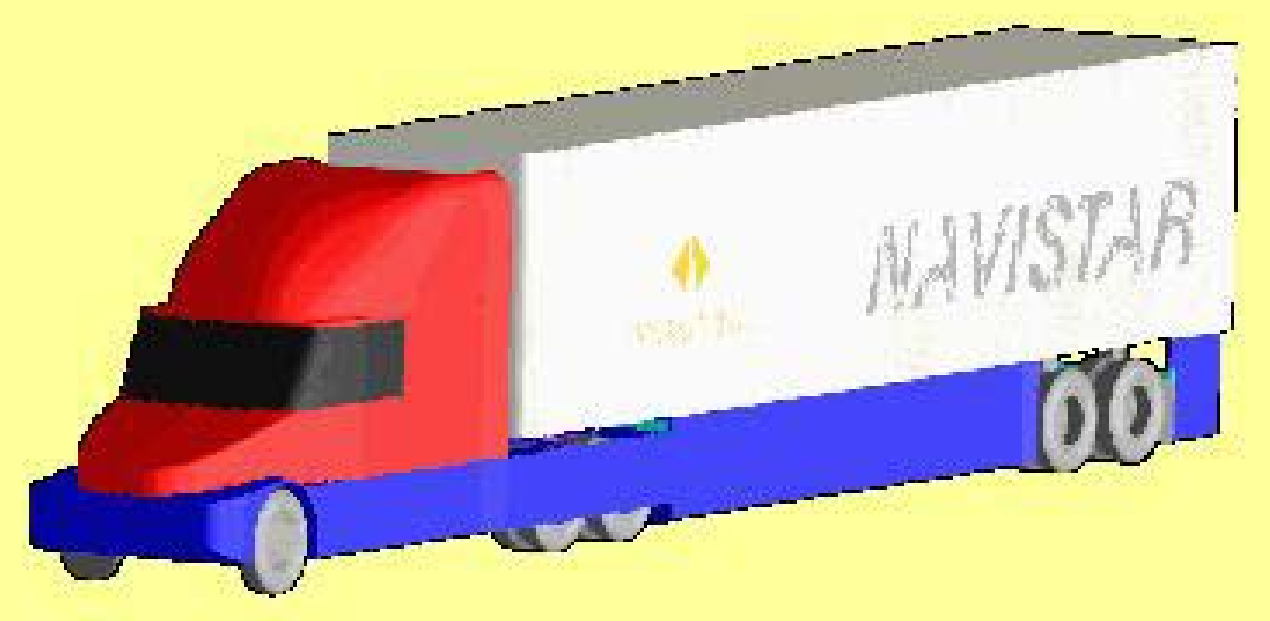




\section{AISDS}
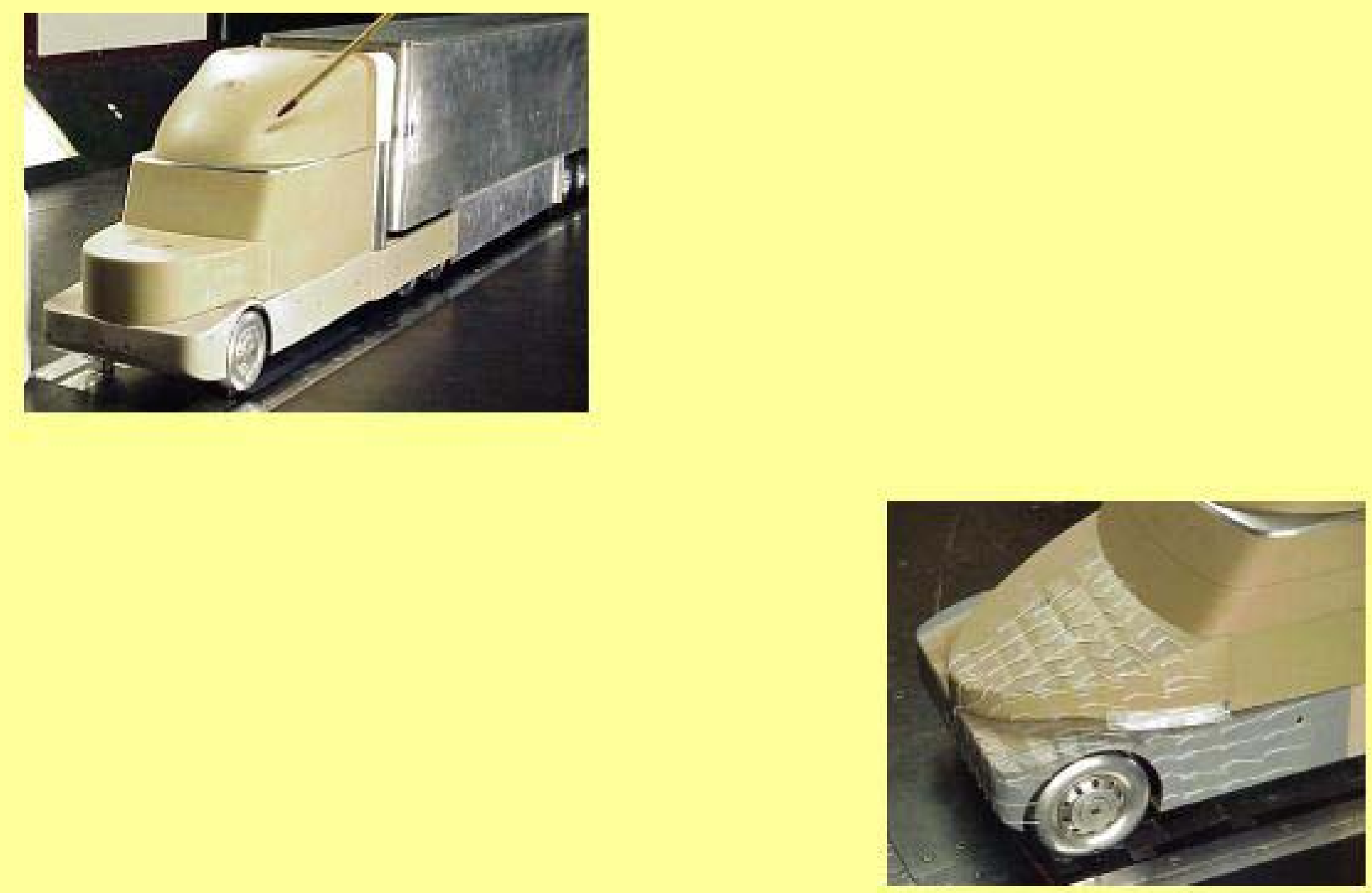

March 28, 2001 


\section{Computational Fluid Dynamics}

- In-House Group Established 2 Years Back

- Usage

- Pro-active

- Re-active

- External Flows

- PowerFlow

- Internal Flows

- Fluent

- Total Vehicle

- Fluent

- Correlation with Wind Tunnel Results Being Established 


\section{CFD Correlation}

- Numerous Simulations Completed

- Drag results don't match yet Consistently

- Good qualitative results

- Good match with pressures from wind tunnel

- Working with software vendor to resolve accuracy issues

- Comparison using GTS Model

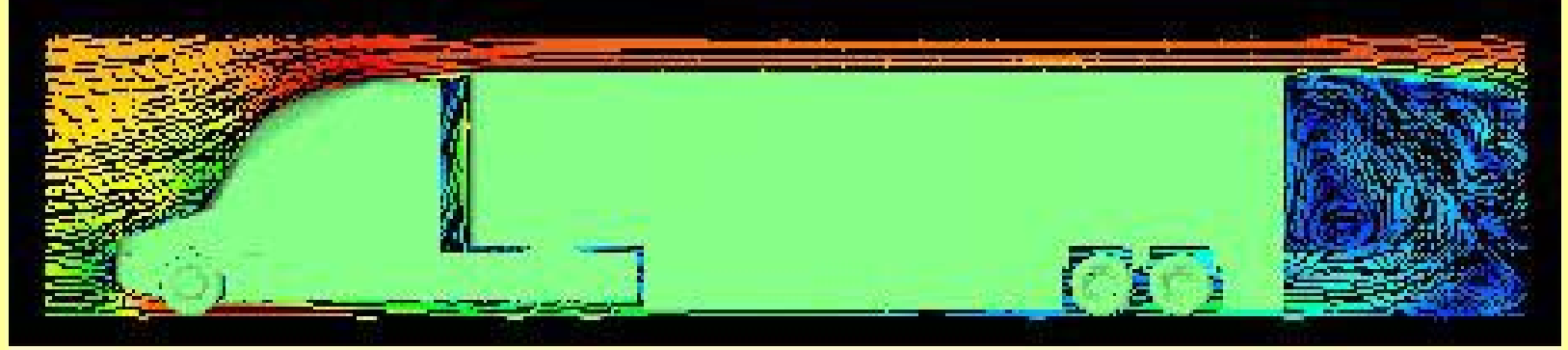




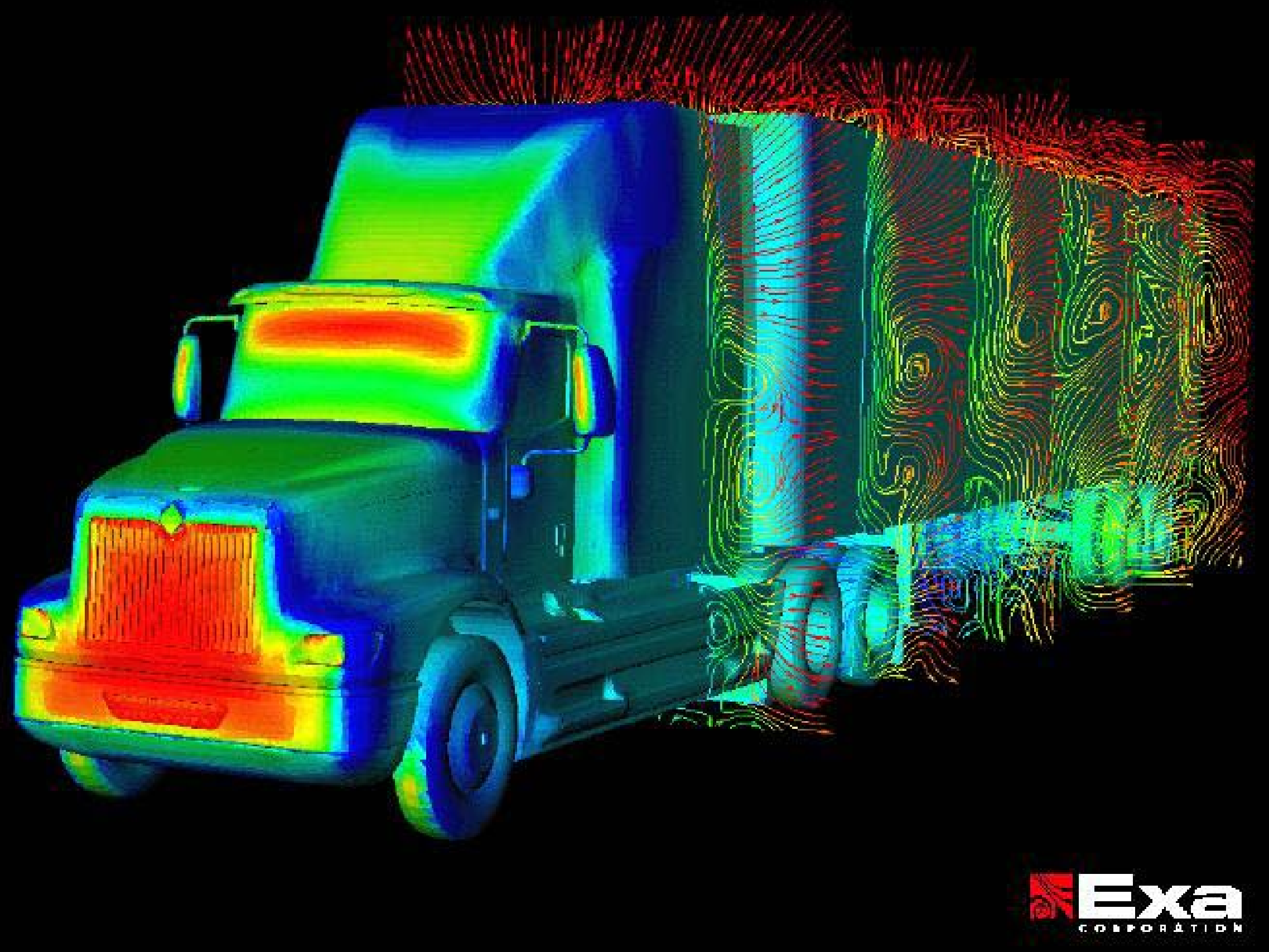




\section{CFD Pro-Active/Re-Active}

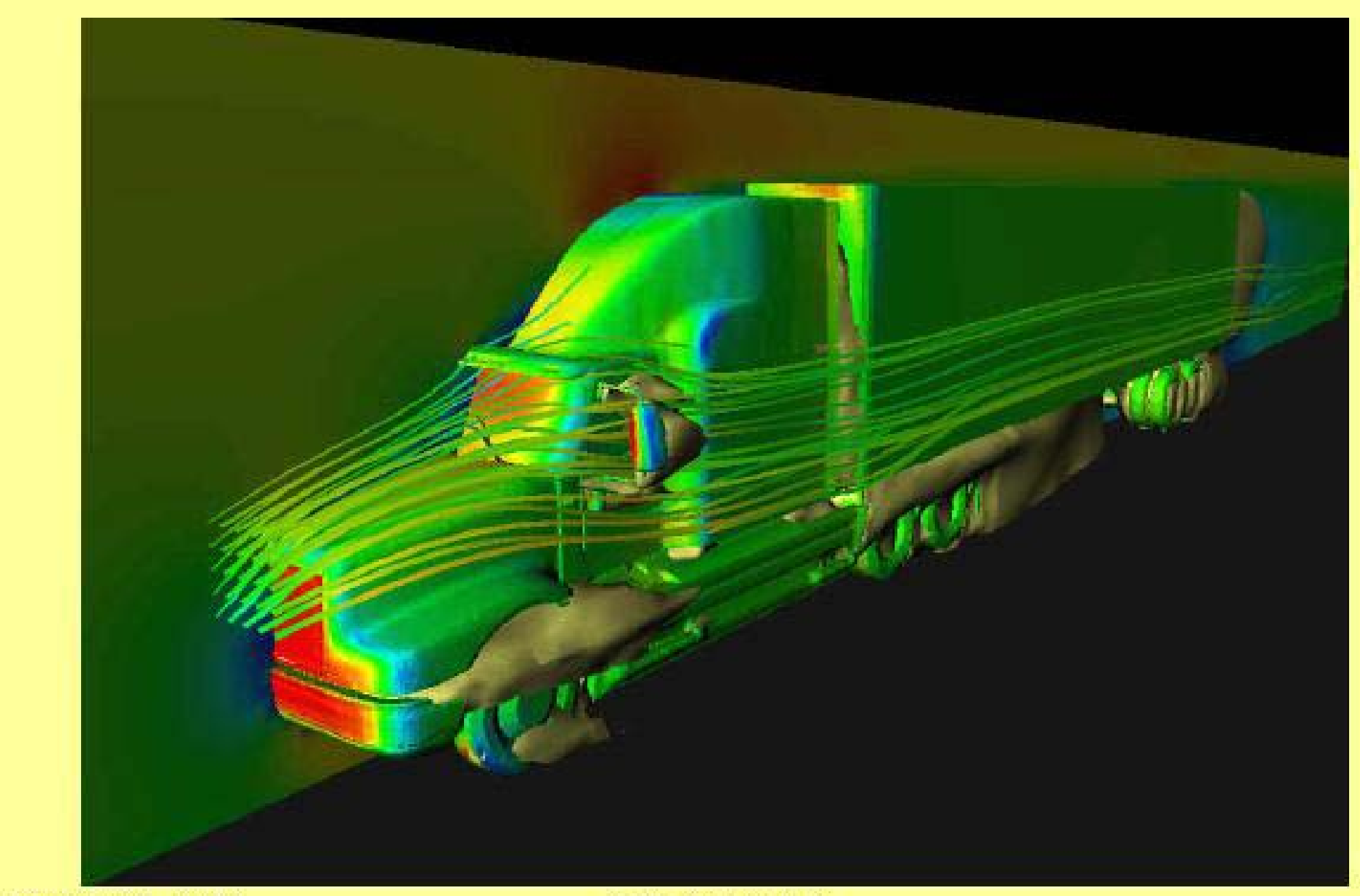




\section{External Agencies}

- DOE

- Looking for Near Term Benefits

- Access to the Code

- Results on GTS Incl. Cd Comparison

- Suggestions for Improving Truck Aerodynamics

- Involvement of Software Vendors

- SAE

- Organizing Upcoming Truck \& Bus Meeting

- Numerous Papers

- RFPs 


\section{Roadmap \& Conclusion}

- Near Term

- Establish Cost Effective as well as Sufficiently Accurate CFD Methodology for Truck Configuration Evaluation

- Continue CFD correlation studies using PowerFlow 3.2

- Integration of CFD in our Future Product Design Process

- Continue Exploring Technologies for Drag Reduction as well as to Improve our Methodology as well as to Drag reduction

- DOE Efforts

- Software Vendors

- Joint Efforts with Various Other Agencies

- We Believe These Efforts along with Continuous Encouragement from Leadership Team will not only Keep our Products BIC, but also will Help us Move towards the 21st Century Truck Initiative Projection on Aerodynamic Drag. 


\section{PACCAR'S VEHICLE-AERODYNAMICS DEVELOPMENT PROGRAM}

28 March 2001

PACCAR and Heavy-vehicle Manufacturing Divisions

- Business profile

- Truck divisions

\section{Product Performance Assurance}

- Development stages: benchmarking and target setting, advanced concept, prototype validation, production confirmation

- Evaluation/assurance tools: CFD, wind tunnel, vehicle/track

CFD Modeling Tools

- Star-CD

- EXA

- Others

Wind Tunnel Experiment Tools

- Sub-scale test

- Full-scale test

Vehicle/Track Confirmation Tools

- Pressure test

- Force test

- Energy (fuel) test 


\section{PACCAR'S INPUTS TO DOE'S HEAVY VEHICLE AERODYNAMIC DRAG PROGRAM}

\section{March 2001}

\section{Helpful Tools and Techniques}

1. Evaluation of selected CFD codes (NASA, DOE, and commercial) suitable for analyses of heavy vehicles. Advice in their capabilities and limitations (including accuracy and computing speeds vs. costs) for specific applications.

2. Similarly, advice in available technologies and techniques for wind-tunnel experiments and road evaluations that would facilitate the development of more-aerodynamic vehicle models.

3. Development of new reference-vehicle model, replacing the existing GTS model, that would be more similar in geometry to the vehicles on the road today.

4. Analysis and correlation of the above reference model for CFD, wind-tunnel, and full-scale vehicle experiments to demonstrate drag-reduction practices and suggest design guidelines.

\section{Helpful Analyses for Specific Vehicle Areas and Components}

5. Effect of trailer gap and gap-reduction devices/treatments, and the integration between tractor and trailer for drag reduction.

6. Underbody airflow as influenced by ground proximity.

7. Wheel-well effect and splash/spray (also fouling) performance.

8. Under-hood (engine compartment) airflow and interaction with external aerodynamics for optimized energy conservation.

9. Base-pressure distribution around the vehicle for not only drag evaluation but also in-cab flow optimization (HVAC and exhausters) and wind-noise reduction.

10. Wind/yaw-angle effect on aerodynamic performance of vehicles and components. 


\section{Task Overview}

\$ Evaluation of current Technology

\$ Support of Testing Activities

\$ Liason with industry

$\$$ Other near term aero activities

+ Removal of mirrors 


\section{Evalutaion of Current Technology}

\$ Selected Public Codes

+ USM3D - unstructured, multi-block, preconditioned

+ Overflow -overset multi-block, preconditioned

+ INS3D -overset multi-block, incompressible

\$ Selected Commercial Codes

+ Fluent

+ Star CD

+ EXA Powerflow 


\section{Evalutaion of Current Technology}

\$ Agreements have been reached with holders of each code

\$ EXA, Adapco and Fluent will donate computing power

$\$$ Overflow and INS3D will run on Athlon PC ( 500M RAM)

$\$$ Need PC cluster or supercomputer for USM3D

$\$$ Waiting for 'as built' geometry 


\section{Support of Testing Activities}

\$ Geometric modeling

\$ Assisted in pressure tap location

\$ Assisting in planning and execution of vortex generator tests 


\section{Liason with Industry}

\$ Conversations with Frieghtliner, Mack and PACCAR reveal two strong themes.

+ Efforts to provide validation data should be continued and expanded.

" High Reynolds number tests

" Calibrated road tests

" Underhood data

+ Convert analysis focus to design focus

" Accelerated convergence

" Solution perturbations

" Parametric geometry methods

" Optimization methods or design space navigation 


\section{Other near term aero activities}

\$ Removal of mirrors

" Fuel savings of 1\% to 3\%

" Significant increase in safety

+ Multi-agency meeting to discuss roles.

+ DOT has started visibilty studies 


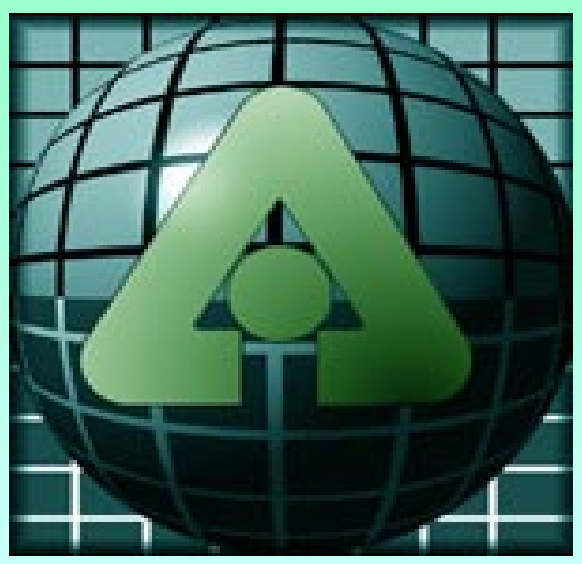

Heavy Vehicle Aerodynamic Drag Working Group Meeting

Results with a Commercial Tool

David P. Weber

Tanju Sofu

March 28, 2001

Argonne National Laboratory 


\section{Underhood Thermal Management}

* Cooperative Research and Development Agreement with adapco, GM, Ford, DaimlerChrysler and ORNL

- Fan, Radiator and Heat Transport System Models

- Improved Numerics in CHAD

- Radiation Heat Transfer Model

- Verification \& Validation

* Hybrid Vehicle Thermal Management with adapco

- Exploratory Use of STAR-CD for Hybrid Vehicles

- Comprehensive Model of Underhood Environment

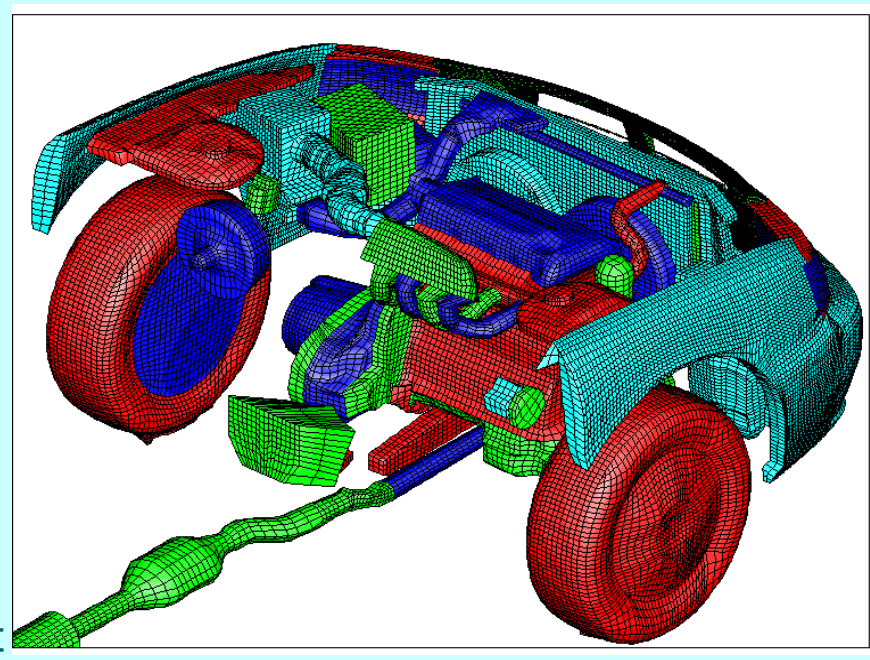

- Validation through utilization of ANL Hybrid Vehicle Experimental Test Facility

* Virtual Reality for Examination of Results

- Application of High Performance Application of High Performance Computing to Automotive Design and Manufacturing

Argonne National Laboratory 


\section{MODEL DESCRIPTION}

- The model consists of flow domain that approximates a wind-tunnel like environment for a stationary car (ground moving)

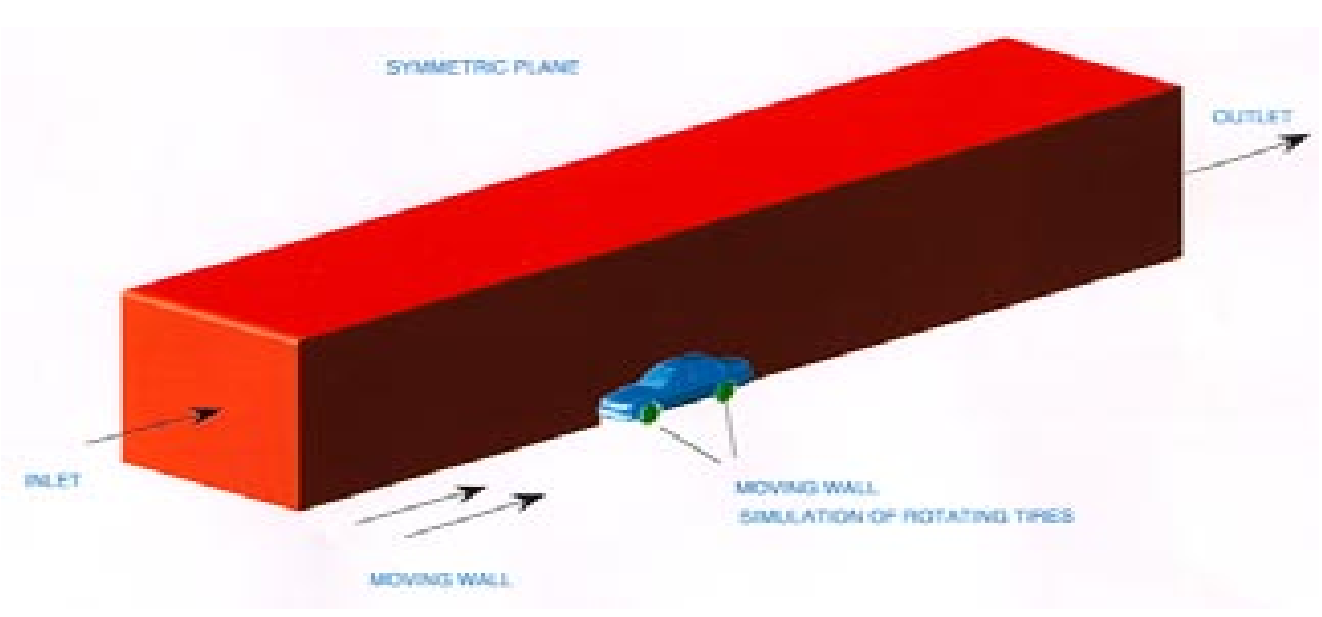

Boundary Conditions for Vehicle Speed of $60 \mathrm{~km} / \mathrm{h}$

\begin{tabular}{|l|c|}
\hline Air Inlet Velocity & $16.67 \mathrm{~m} / \mathrm{s}$ \\
\hline Air Inlet Temperature & $20^{\circ} \mathrm{C}$ \\
\hline Ambient Pressure & $1.0 \mathrm{~atm}$ \\
\hline Wheel Speed & $500 \mathrm{rpm}$ \\
\hline Coolant Flow Rate & $1.0 \mathrm{~kg} / \mathrm{s}$ \\
\hline Coolant Inlet Temp. & $85^{\circ} \mathrm{C}$ \\
\hline Fan Speed & $1350 \mathrm{rpm}$ \\
\hline
\end{tabular}




\section{MODEL DESCRIPTION (cont.)}

- Entire vehicle (including the passenger

compartment) is

represented to model various interrelated airflow phenomena and thermal activity

- Combining 3-D flow and heat transfer into one large model allows assessment of inherent interdependency of components

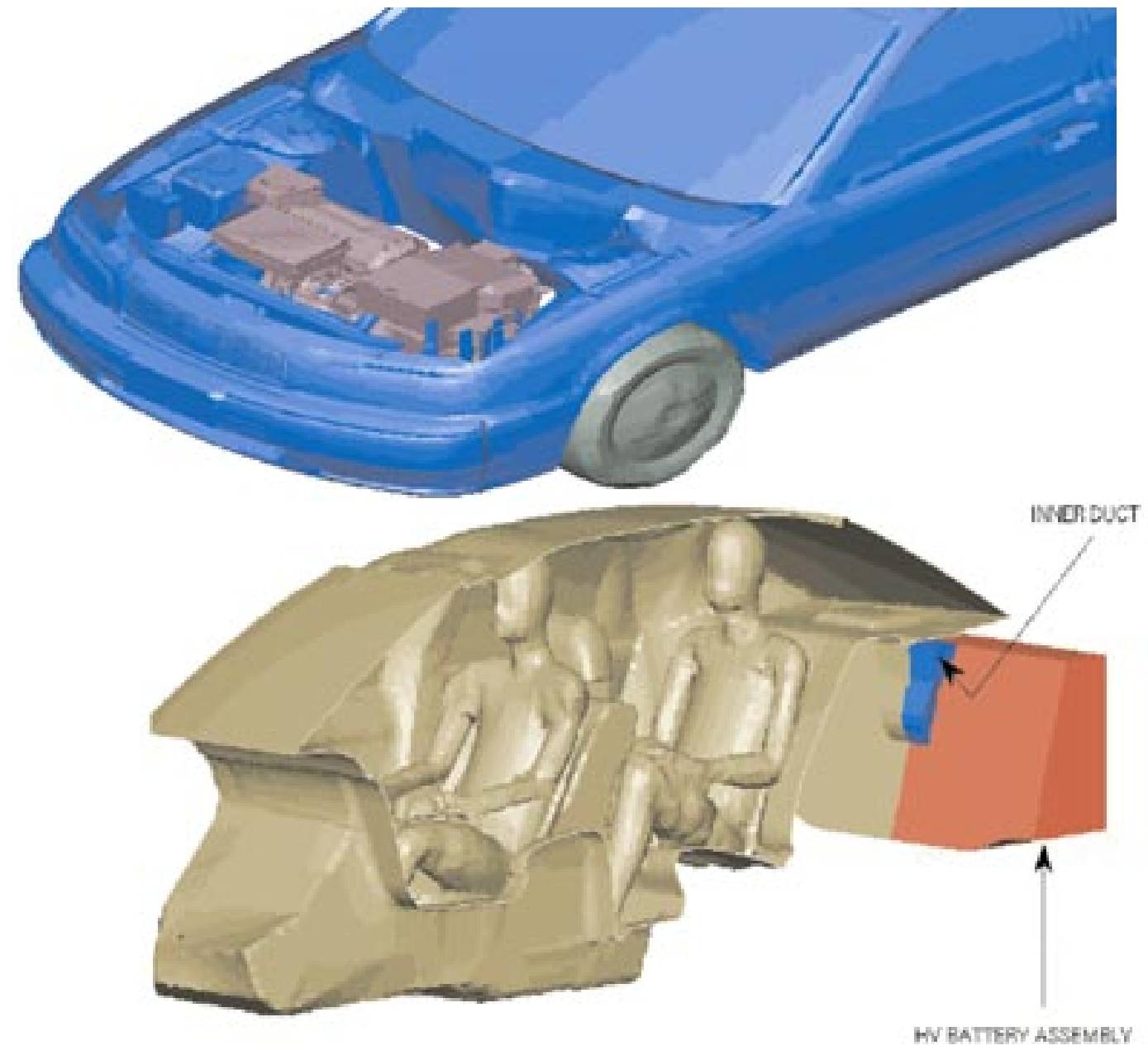


PROSTAR 3.05

VELOCITY

$\mathrm{m} / \mathrm{s}$

STATIC REFERENCE

"PRESENTATION GRID*

16.00

15.20

14.40

13.60

12.80

12.00

11.20

10.40

9.600

8.800

8.000

7.200

6.400

5.600

4.800

4.000

3.200

2.400

1.600

.8000 A

i.

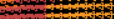
$20 x+201$

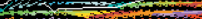

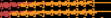

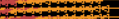

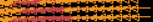

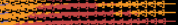

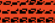

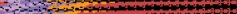

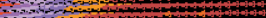

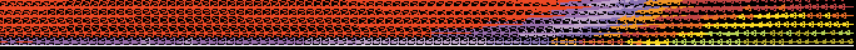
HYBRID VEHICLE

Underhood/Interior/Battery Compartment Model, 60kph Case

$Y=250 . \quad \mathrm{mm}$ 


\section{Drag Comparisons}

New Model Turn Around Time: 2-7

\begin{tabular}{|c|c|c|c|c|}
\hline Vehicle & Description & Case & Drag & Difference \\
\hline \multirow{6}{*}{ A-Class } & \multirow{6}{*}{ Medium Detail } & Baseline- 1999 v3050 & 0.300 & $1.4 \%$ \\
\hline & & Baseline-Edges & 0.307 & $1.0 \%$ \\
\hline & & Baseline-No Edges & 0.306 & $0.6 \%$ \\
\hline & & Experiment & 0.304 & \\
\hline & & Mod 2 & 0.278 & $3.0 \%$ \\
\hline & & Experiment & 0.270 & \\
\hline \multirow{2}{*}{ Large Sedan } & \multirow{2}{*}{ Low Detail } & EZAero & 0.281 & $4.1 \%$ \\
\hline & & Experiment & 0.270 & \\
\hline \multirow{2}{*}{ Benchmark 1} & \multirow{2}{*}{$\begin{array}{l}\text { Low Detail } \\
\text { Small Vehicle }\end{array}$} & EZAero & 0.212 & $0.5 \%$ \\
\hline & & Experiment & 0.213 & \\
\hline \multirow{2}{*}{ Benchmark 2} & \multirow{2}{*}{$\begin{array}{l}\text { Low Detail } \\
\text { Slanted Rear }\end{array}$} & EZAero & 0.280 & $1.4 \%$ \\
\hline & & Experiment & 0.276 & \\
\hline \multirow{2}{*}{ Truck } & \multirow{2}{*}{$\begin{array}{l}\text { Medium Detail } \\
6 \text { million cells }\end{array}$} & EZAero & 0.815 & $0.6 \%$ \\
\hline & & Experiment & 0.820 & \\
\hline
\end{tabular}




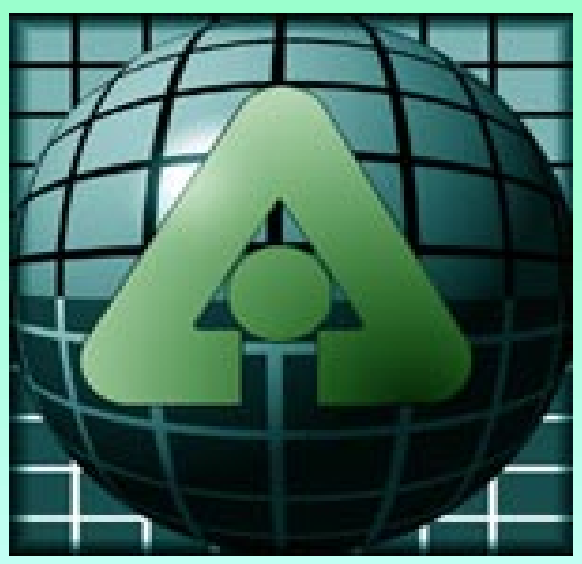

\title{
Heavy Vehicle Simulations with STAR-CD
}

Heavy Vehicle Aerodynamic Drag Working Group Meeting

Lawrence Livermore National Laboratory

\author{
Tanju Sofu
}

March 28-29, 2001

Argonne National Laboratory 


\section{Summary and Outline}

* Results with a commercial tool for two industrial applications

- Commercial Truck Analysis

$\downarrow$ Aerodynamic simulation of reference design

$\downarrow$ Drag coefficient comparisons for three proposed design changes

- Integrated Systems Simulation (aerodynamic and underhood cooling analysis)

* Both simulations are performed by adapco, Inc. using STAR-CD

- "On the road" simulation

$\uparrow$ symmetry boundaries

$\checkmark$ stationary vehicle

$\downarrow$ rolling wheels, ground moving

- Combined 3-D flow and heat transfer in a large model for assessment of inherent interdependency of components

- Blind Pre-Test Analyses 


\section{Commercial Truck - Reference Design}

* Detailed geometric modeling starting from CAD surfaces (full suspension and undercarriage included)

* Unstructured mesh with refinements (10 million cells)

- Near walls

- Under the truck

- In the cabin-trailer gap

- Behind the truck

* Analysis for reference design demonstrates a steady flow field (not oscillating). $\kappa-\varepsilon$ model is used. 


\section{Commercial Truck - Reference Design}

Aerodynamic simulation (frontal area $9.44 \mathrm{~m}^{2}$ )

* Drag coefficients:

\begin{tabular}{|c|c|c|}
\hline & STAR-CD & Wind Tunnel \\
\hline $0^{\circ}$ Yaw & 0.815 & 0.82 \\
\hline $10^{\circ}$ Yaw & 1.613 & 1.51 \\
\hline
\end{tabular}

- Reported values for $C_{d}$ are raw measurements prior to any correction factors due to the walls of wind tunnel

- Calculated values are for "on the road" simulation (closer to a corrected value)

- Blind Pre-Test Calculations 


\section{Commercial Truck - Case Comparisons}

\section{Calculated Drag Coefficients}

\begin{tabular}{|c|c|c|}
\hline & $0^{\circ}$ Yaw & $10^{\circ}$ Yaw \\
\hline Reference Case & 0.815 & 1.613 \\
\hline $\begin{array}{l}\text { Case 1: } \\
\text { New gap fairing }\end{array}$ & $\begin{array}{c}0.807 \\
\text { (1\% reduction) }\end{array}$ & $\begin{array}{c}1.576 \\
\text { (2\% reduction) }\end{array}$ \\
\hline $\begin{array}{l}\text { Case 2: } \\
\text { Full aerodynamic kit }\end{array}$ & $\begin{array}{c}0.751 \\
\text { (8\% reduction) }\end{array}$ & $\begin{array}{c}1.472 \\
\text { (9\% reduction) }\end{array}$ \\
\hline $\begin{array}{l}\text { Case 3: } \\
\text { Idealized truck design }\end{array}$ & $\begin{array}{c}0.583 \\
\text { (29\% reduction) }\end{array}$ & $\begin{array}{c}0.994 \\
\text { (38\% reduction) }\end{array}$ \\
\hline
\end{tabular}




\section{Integrated Systems Simulation}

* A two-axle compact truck

* Aerodynamic and underhood analysis

* Unstructured mesh with refinements near walls, under the truck, under the hood, and behind the truck (20 million cells)

* "On the road" simulation at $85 \mathrm{~km} / \mathrm{h}$

* Geometric details included (full suspension and undercarriage)

* Calculated drag coefficient $=0.583$

* Model for underhood flow and thermal analysis

- Radiator as a dual-stream, single phase heat exchanger

- Condenser as a dual-stream, two-phase heat exchanger

- Fan rotation modeled with implicit multiple reference frames

- Effect of bottom cover on underhood cooling estimated 


\section{Turbulence Modeling Options}

* $\kappa-\varepsilon$ models

- Standard model (high Re form in conjunction with 'law of the wall' representation of flow, heat and mass transfer within boundary layers)

- Damping function based low-Re model ( $\kappa$ and $\varepsilon$ equations solved everywhere, including the boundary layers)

- Renormalization group (RNG) variant and Chen's version (employs both the production and dissipation time scales in closing the $\varepsilon$ equation)

- Alternative non-linear (quadratic and cubic) $\kappa-\varepsilon$ models for High- and LowRe flow

* Two-layer models. Near wall flow is simulated via either

- $\kappa$ equation and an algebraic prescription for $\varepsilon$ (single equation model)

- Mixing length model (turbulent viscosity from Prandtl's mixing length hypothesis) 


\section{Turbulence Modeling Options (cont.)}

* Large Eddy Simulation

- large-scale turbulent motion is explicitly solved while small scale eddies are approximated by a model (sub-grid scale Reynolds stresses are also included)

* V2F (Stanford) model through a license from Cascade Tech. Inc.

- Full low Re model (valid throughout the flow domain including the viscous-affected near-wall region)

- Accurate prediction of flow-separation, skin friction, and heat transfer

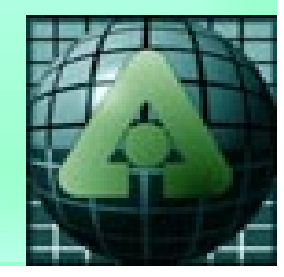




\section{Conclusions}

* Aerodynamic analyses with STAR-CD for two commercial trucks

- Rapid model development with semi-automated mesh generator

- Full geometric details for a first-principles based analysis

- Good agreement for drag coefficient estimations

- Case comparisons for design improvements

* Integrated aerodynamic and underhood cooling analysis for the entire vehicle

- A systems simulation capability for studying load conditions

- Assessment for interdependence of underhood components

- Tool for identifying critical components and their long-term reliability

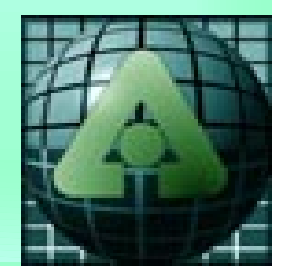




\title{
Draft of SAE Paper 2001-01-2072 \\ to be Presented at the SAE Government/Industry Meeting, DOE OHVT-Systems Session, Dr. Sid Diamond, Chairman \\ Washington, DC \\ May 16, 2001 \\ Draft date: April 2, 2001
}

\section{Advanced Aerodynamic Devices to Improve the Performance, Economics, Handling and Safety of Heavy Vehicles}

\author{
by \\ Robert J. Englar, Principal Research Engineer \\ Georgia Tech Research Institute \\ Aerospace, Transportation \& Advanced Systems Lab \\ Aerodynamics \& Acoustics Branch \\ Atlanta, GA 30332-0844 \\ (770) $528-3222$ \\ (770) 528-7077, Fax \\ bob.englar@gtri.gatech.edu
}

\begin{abstract}
Research is being conducted at the Georgia Tech Research Institute (GTRI) to develop advanced aerodynamic devices to improve the performance, economics, stability, handling and safety of operation of Heavy Vehicles. The objective of this program is the application to tractor/trailer configurations of pneumatic (blown) aircraft technology previously developed and flight-tested by GTRI personnel. Earlier GTRI experimental results using blowing on a streamlined car wind-tunnel model had shown drag coefficient reductions of up to $35 \%$, plus lift increases or reductions (downloads) of 100-150\%, depending on the blowing location and the force direction desired. Also demonstrated was the ability to control aerodynamic moments about all three axes without any moving control surfaces.

Recent wind-tunnel investigations of a generic Pneumatic Heavy Vehicle model with blowing slots on both the leading and trailing edges of the trailer have been conducted under contract to the DOE Office of Heavy Vehicle Technologies. These results have shown overall aerodynamic drag reductions on the Pneumatic Heavy Vehicle of over 50\% using only 1 psig blowing pressure in the plenums, and over $80 \%$ drag reductions if additional blowing air were available. Blowing-reduced drag coefficients of less than half that of a Corvette coupe were measured experimentally. Furthermore, an increase in drag force for braking was made possible by blowing different slots. Lift coefficient increase was generated by blowing only the top slot, while downforce was produced by blowing only the bottom. Also, side force and yawing moment were generated on either side of the vehicle and directional stability was restored by blowing the appropriate side slot.

These experimental results and the predicted full-scale payoffs are presented in this paper. Also provided is an introduction to this new aerodynamic/automotive technology, plus discussion of additional applications to conventional commercial autos, buses, and Sport Utility Vehicles, as well as to Formula 1 and other race cars.
\end{abstract}




\section{INTRODUCTION}

Users of heavy trucks such as tractor/trailer combination vehicles face a number of less than optimum operating conditions. Despite significant reductions in drag coefficients in the latest generation of tractors, these Heavy Vehicles (HVs) remain "draggy" compared to much more streamlined automobiles. This is due in part to practical limitations on: physically providing a long smooth aft surface such as a boat tail to prevent flow separation and turbulence at the rear of the trailer; completely sealing the gap between the tractor and the trailer; and smoothing the underbody of the vehicle. In addition, front radiators have not been optimized from a drag-reduction standpoint. Typical drag coefficient values for a variety of HVs can range from 0.65 to 0.9 (from Reference 1). Figure 1 shows the significant fuel savings that can result if the drag coefficient can be reduced. It has been estimated by engineering personnel of the American Trucking Associations (ATA) that, if applied to today's US Heavy Vehicle fleet operating on level roads, these drag reductions approaching 35\% could result in roughly 1.2 billion gallons of fuel saved per year. Extrapolated, a 50\% drag reduction could save over 1.7 billion gallons of diesel each year. A further result of aerodynamically "dirty" vehicles is the production of splash and spray, a nuisance to motorists and truck drivers alike, as well as turbulence in the vicinity of large vehicles, which is disturbing to passenger car drivers. These shortcomings are explained further in the ATA Statement of Need for Improved Heavy Truck Aerodynamics, Reference 2.
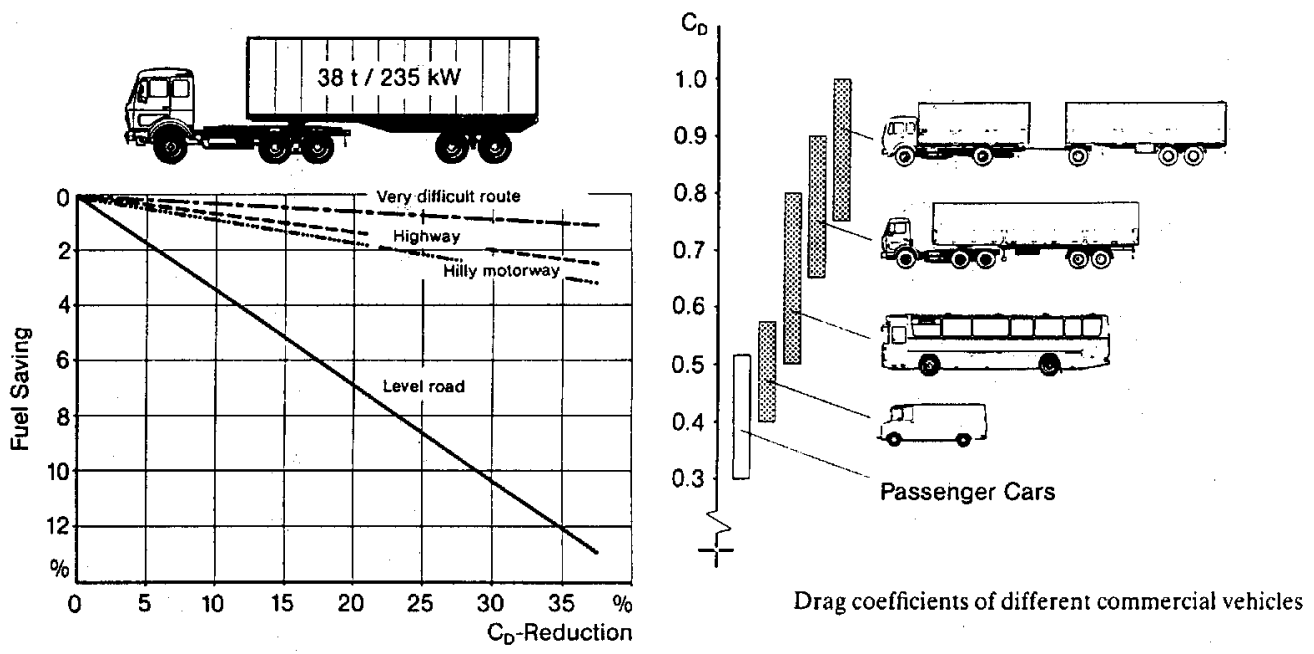

Influence of drag on fuel consumption of a 38-tonne semitrailer

Figure 1 - Effect of Drag Coefficient Reduction on Fuel Consumption (from Ref. 1)

Considerable recent interest has arisen in techniques to reduce Heavy Vehicle drag to improve highway operating efficiency, primarily because the drag force rises with the square of the vehicle speed while the required horsepower to overcome that drag increases with the cube of the truck's velocity. With fuel prices rising dramatically recently, drag reduction is thus a vital concern to the trucking industry. However, there is significant reason to believe that appreciable additional gains can also be had by careful control of aerodynamic forces and moments other than drag, which for the most part have been ignored in current truck design and operation. For instance, the creation of lift on the vehicle (effective weight reduction) can unload the tires and reduce rolling resistance, while creation of negative lift or downforce can increase "weight" on the wheels and traction, thus increasing braking as well as handling in wet/icy weather. This aerodynamic download can also eliminate hydroplaning. While it has been shown that drag increases greatly due to side wind or yaw angle (Refs. 1 and 3), this also implies increased side force and yawing moment on the trailer, thus reducing its directional 
stability and safety. Safety, stability and handling can be enhanced by blowing control of side loads and moments on these Heavy Vehicles if caused by side winds, gusts or other vehicles passing. This pneumatic concept may also help to eliminate the jack-knifing problem if resulting from extreme wind side loads on the trailer. Lastly, there are instances where additional drag increase is desirable, such as steep downhill operations in mountains, or sudden need for emergency braking from high speed.

Based on the above considerations, it should be quite desirable to develop aerodynamic devices that could achieve two or more of these potential gains while requiring little mechanical restriction or impact on vehicle operation. Recent aerodynamic research (Reference 3) at GTRI's Aerospace, Transportation and Advanced Systems Lab has identified significant reductions and/or augmentations of vehicle forces and moments which can be achieved on automotive vehicles by the use of tangential injection of pressurized air into the vehicle's aft boundary layer, strongly modifying the aerodynamic flowfield around that vehicle. Since momentum injection can affect the vehicle's lift, drag, and side force as well as aerodynamic moments, the impact of blowing on the performance, safety, economics and stability appears to offer significant improvements in Heavy Vehicle operation. These potential gains have led to a current research program being conducted at GTRI for the DOE Office of Heavy Vehicle Technologies (Reference 4). A basic description of this program and the novel aerodynamic technology to be employed is provided in Reference 5. Since that paper was presented in June, 2000, two series of wind-tunnel evaluations have been conducted at GTRI to confirm aerodynamic improvements yielded first by novel unblown geometry changes, and second by the addition of blowing to various portions of a Heavy Vehicle model. The objectives of these tests were to verify the blown pneumatic concept's capabilities to: reduce aerodynamic drag for efficiency or increase drag for braking; increase lift (and thus reduce tire rolling resistance) or reduce lift (and thus increase traction and braking); and provide increased lateral/directional stability and safety, all without use of external moving aerodynamic components. The present paper describes the basis of pneumatic aerodynamics and its application to Heavy Vehicles, and then provides details of the two wind-tunnel programs, their results, and possible future applications.

\section{PNEUMATIC AERODYNAMICS}

GTRI researchers have been involved for a number of years in the development of pneumatic (pressurized air blowing) concepts to yield efficient yet mechanically simple means to control and augment or reduce the aerodynamic forces and moments acting on aircraft. This was detailed in References 5, 6, 7, and 8, but will be summarized briefly here to familiarize the reader with the technology. Figure 2 shows the basic pneumatic concept, which has become known as Circulation Control (CC) aerodynamics. Here, an airfoil's conventional mechanical trailing edge device has been replaced with a fixed curved surface and a tangential slot ejecting a jet sheet over that surface. That jet remains attached to the curved surface by a balance between sub-ambient static pressure on the surface and centrifugal force (the so-called Coanda Effect, Reference 8). This greatly entrains the external flowfield to follow the jet, and thus enhances the circulation around the airfoil and the aerodynamic forces produced by it. The governing parameter is not angle of attack, but rather the blowing momentum coefficient:

$$
\mathrm{C}_{\mu}=\mathrm{m} \mathrm{V_{ \textrm {j } }} /(\mathrm{q} \mathrm{S})
$$

where $\mathrm{m}$ is the jet mass flow, $\mathrm{V}_{\mathrm{j}}$ the isentropic jet velocity, $\mathrm{S}$ is a reference wing area (or frontal area for a vehicle configuration) and $q$ is the freestream dynamic pressure, $0.5 \rho \mathrm{V}^{2}$, with $\rho$ being the freestream density, not the jet's density. At lower blowing coefficient $\left(\mathrm{C}_{\mu}\right)$ values, augmentation of the aerodynamic lift by a factor of $\Delta C_{1} / C_{\mu}=80$ has been recorded, representing an $8000 \%$ return on the invested momentum (which in a physical sense is also equal to the jet thrust). Familiarity with 


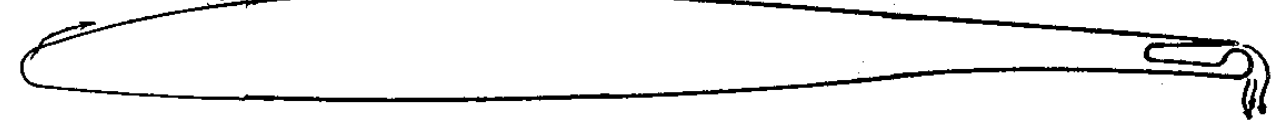

TANGENTIAL BLOWING OVER ROUNDED TRAILING EDGE SURFACE
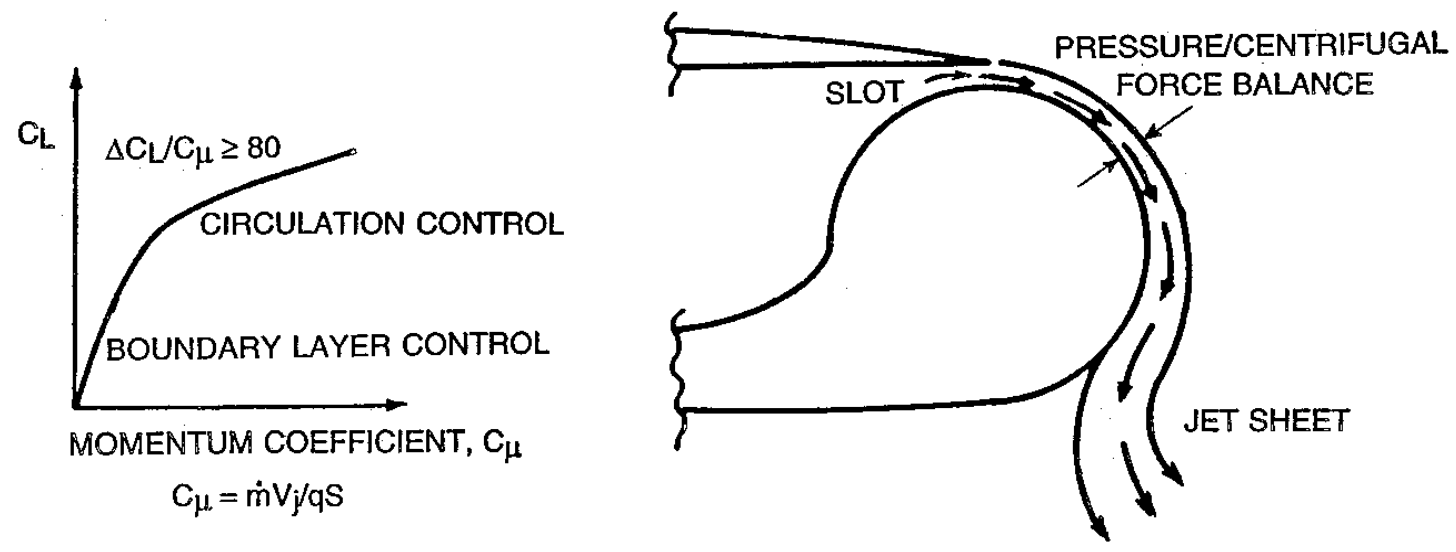

Figure 2 - Basis of Circulation Control Aerodynamics on a Simple 2-D Airfoil

blown aerodynamic systems will remind the reader that this is quite extraordinary: thrust-deflecting Vertical Take-Off and Landing (VTOL) aircraft are fortunate if they recover anything near 100\% of the engine thrust expended for vertical lift. It is because of this high return, and conversely, because of very low required blowing input and associated power required to achieve a desired lift, that Circulation Control airfoils appear very promising for a number of applications. The A-6/CC Wing Short Take Off \& Landing (STOL) flight demonstrator aircraft, Figure 3 and Reference 6, showed the STOL performance listed, but also suggested capabilities very useful to ground vehicles: during short takeoff, it demonstrated very high lift and reduced drag, while in the approach/landing mode, high lift with high drag was shown.

These advantages led to the application of this pneumatic concept to improve the aerodynamics of an already streamlined car (Reference 3 ). The resulting large jet turning angle and the curved rear of the vehicle are shown in Figure 4. Significant but distinctly different trends were observed depending upon which portion of the tangential slot was blown. Blowing the full slot produced the large jet turning in Figure 4, and drag increases of greater than $70 \%$, showing potential for pneumatic aerodynamic braking. Blowing only the outside corner of the slot weakened the corner vortex rollup, lessened aft suction, and reduced drag by as much as 35\% (refer back to Figure 1 for representative fuel savings). Blowing the aft slot also yielded a lift increase of $170 \%$. One can envision a similar slot applied to the lower surface that could yield negative lift or positive down force instead. This concept has been patented by GTRI and verified by a similar installation on a model of a European Formula 1 race car (Reference 5). 


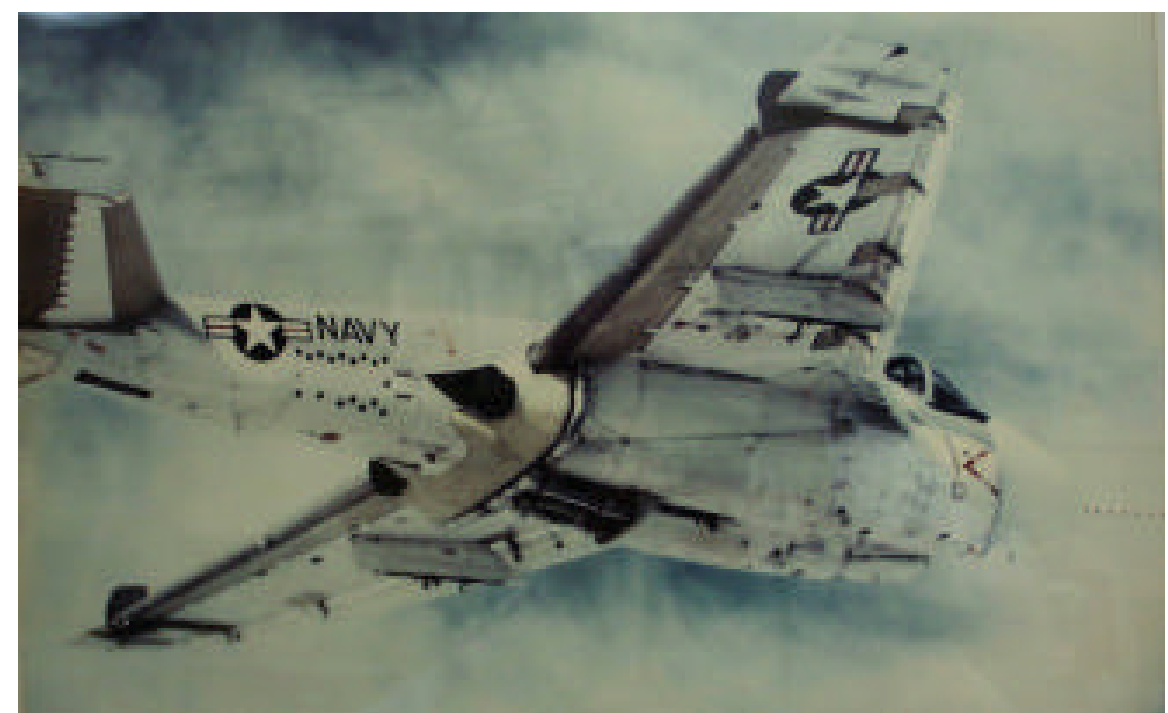

Flight Test Results: $140 \%$ Increase in Usable Lift Coefficient, $\mathrm{C}_{\mathrm{L}}$ 30-35\% Reduction in Takeoff \& Approach Flight Speeds 60-65\% Reduction in Takeoff \& Landing Ground Rolls 75\% Increase in Liftable Takeoff Payload Confirmation of Full-Scale Blown CCW Operation

Figure 3 - A-6/CCW STOL Flight Tests Confirming Pneumatic Devices for Aerodynamic Force Augmentation or Reduction

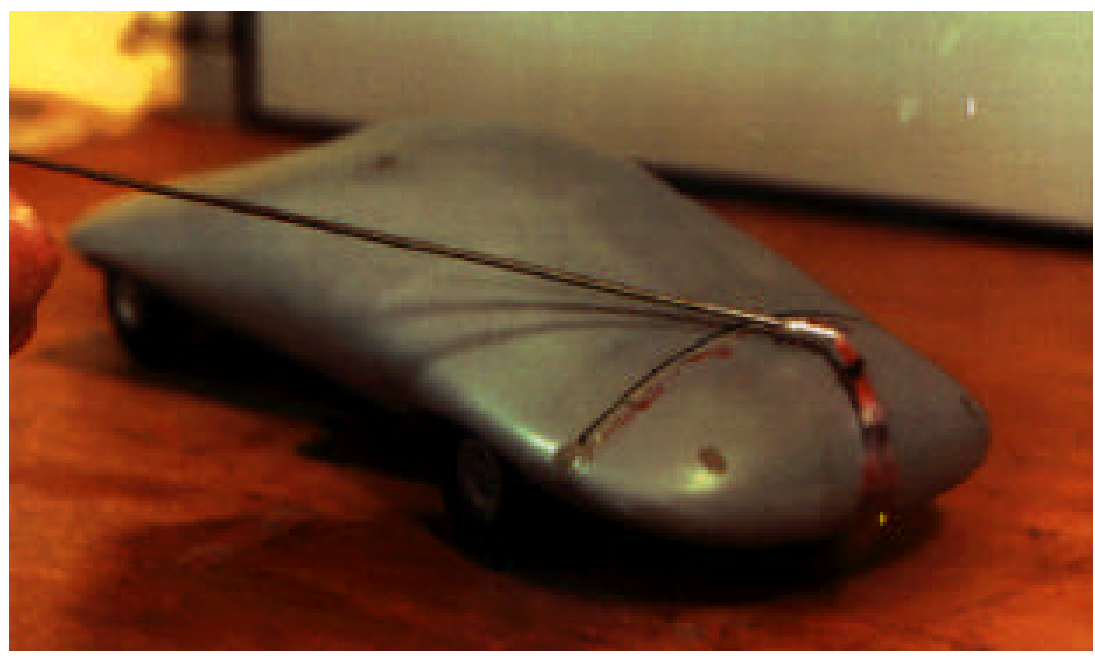

Figure 4 - Experimental Confirmation of Pneumatic Technology on a Streamlined Car; Aft View showing Blown Jet Turning

\section{DOE PNEUMATIC HEAVY VEHICLE PROGRAM}

Based on the above results, a research program was funded at GTRI by the Department of Energy's Office of Heavy Vehicle Technologies (Reference 4). The goal was to apply this pneumatic technology to tractor-trailer configurations to develop an experimental proof-of-concept evaluation that would hopefully lead to an on-the-road demonstration on an operating blown Pneumatic Heavy 
Vehicle (PHV). Portions of that effort, including a preliminary feasibility study, pneumatic Computational Fluid Dynamics (CFD) analyses, and design of baseline and pneumatic wind-tunnel configurations, have been completed and were reported in Reference 5. Figure 5 shows a possible schematic of a generic Pneumatic Heavy Vehicle with tangential blowing slots on each of the trailer's aft edges as well as blowing on the rounded upper leading edge of the trailer.

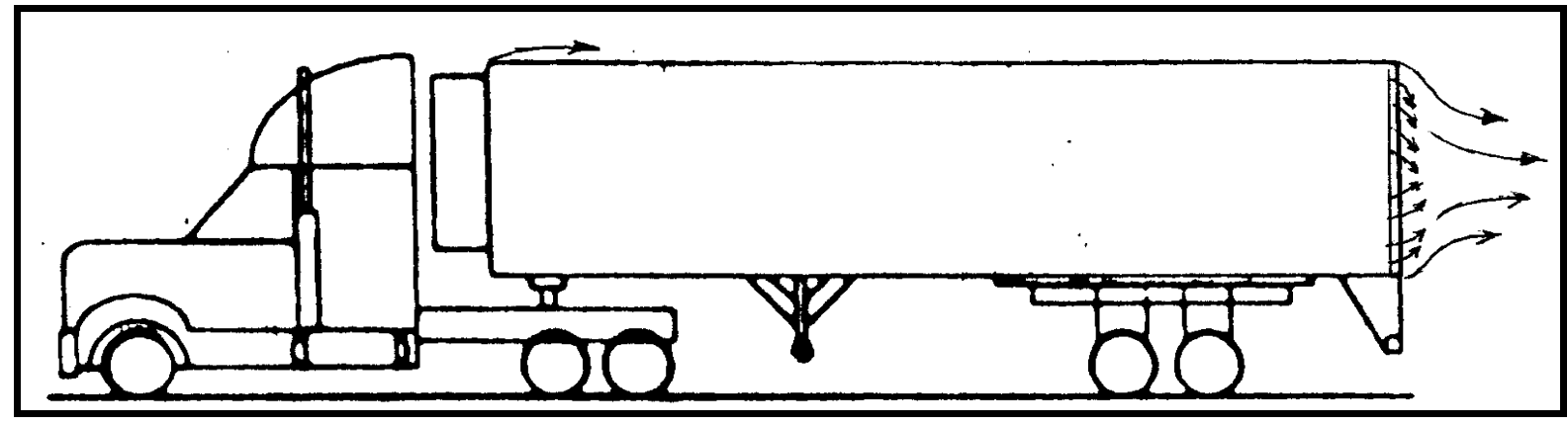

Figure 5 - Schematic of Application of GTRI Pneumatic Aerodynamic Technology to Heavy Vehicle Trailer, Showing 4 Aft Blowing Slots and Upper Leading-Edge Blowing Slot

\section{Phase I Wind-Tunnel Evaluations of Baseline Unblown HV}

To serve as a reference data base and to investigate initial non-blown aerodynamic improvements, a Phase I baseline wind-tunnel test was conducted. For this, a baseline model was needed to act as a standard prior to the planned blowing tests, and as a basis upon which to install the pneumatic model configuration. A team of current researchers working for DOE on the HV aerodynamic drag problem had determined that an existing generic Heavy Vehicle configuration, the Ground Transportation Systems (GTS) vehicle (Reference 9), was quite appropriate. It is sketched in Figure 6, and is actually representative of a faired cab-over-engine vehicle based on the Penske racing team's car carrier, before the blowing modifications shown. As such, it is relatively generic and independent of the numerous and varying cab roof fairings employed on a number of current Heavy Vehicles.

Full Scale Vehicle: $W=8.5^{\prime}, \mathrm{H}=13.5^{\prime}, \mathrm{L}_{\mathrm{TRALER}}=48^{\prime}, \mathrm{L}_{\mathrm{RIG}}=65^{\prime}$; at $\mathrm{V}=70 \mathrm{mph}, \mathrm{Re}_{\mathrm{TI}}=29.3 \times 10^{6}$

Model:

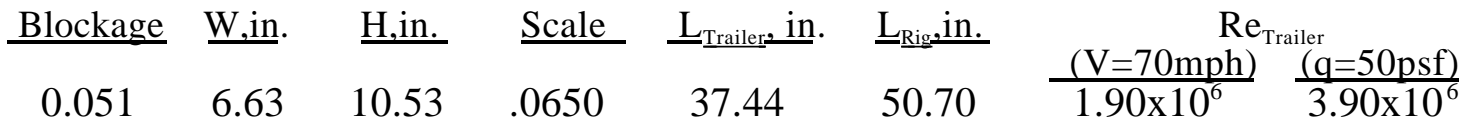

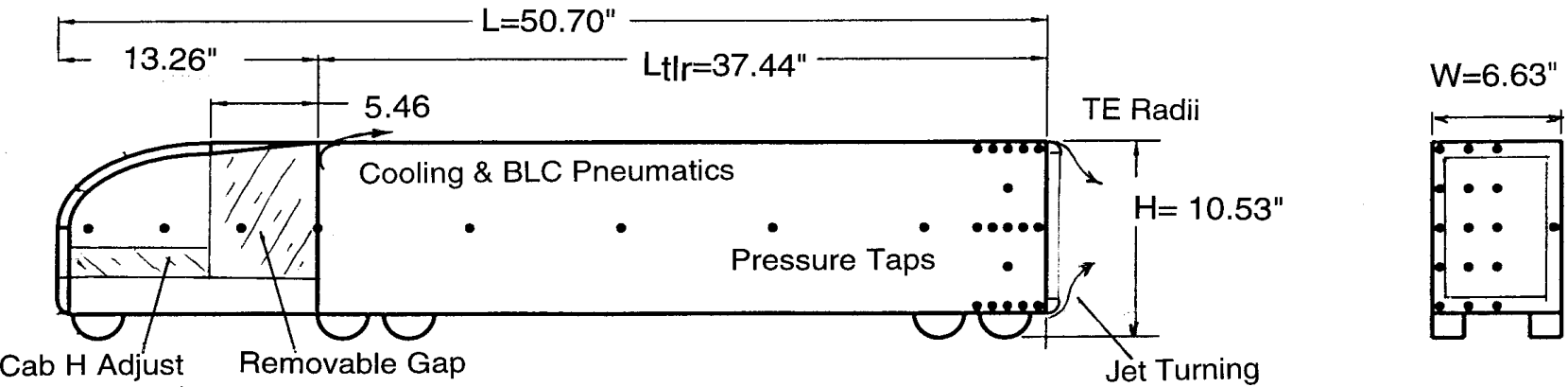

Figure 6- GTRI 0.065-scale Baseline GTS \& PHV Wind-Tunnel Model and Variables 


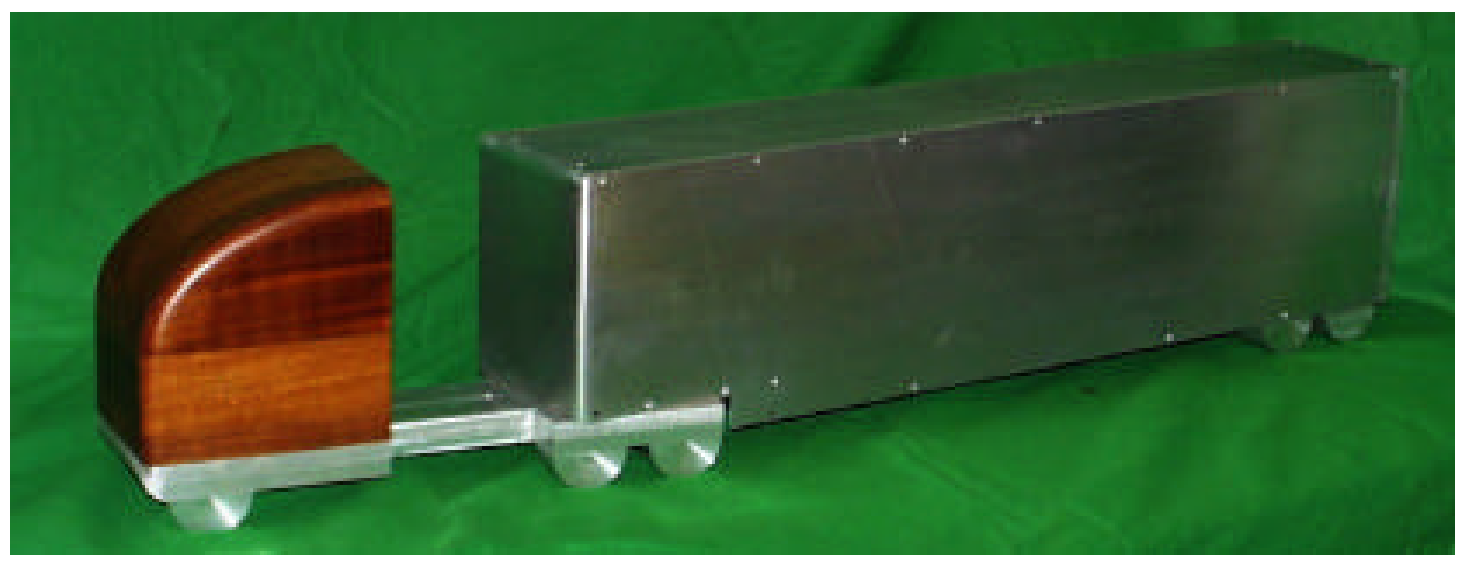

Full-Height Tractor and Yawed Trailer with Rounded Leading Edge and Square Trailing Edge

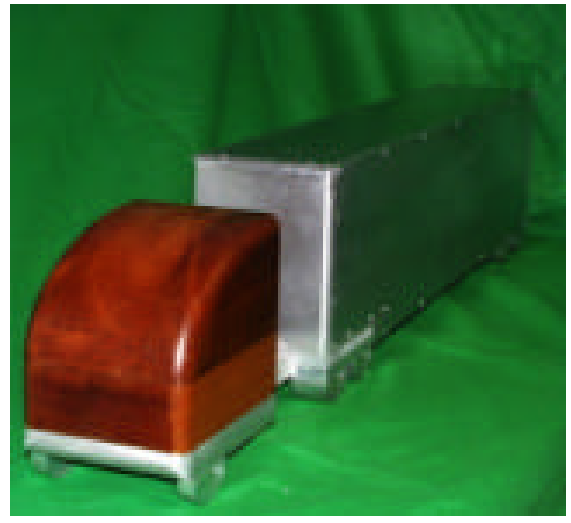

Shorter-Height Tractor, Unyawed Trailer

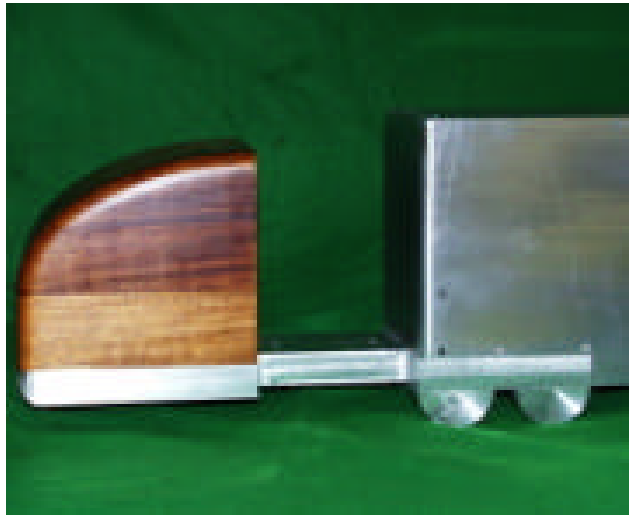

Front Wheels removed

\section{Figure 7 - Photos of Various Configurations of GTRI's Baseline GTS Model, Full Open Gap}

Before being fabricated as a valid wind-tunnel model, issues such as model size, wind tunnel blockage and test Reynolds number were important. The test model was scaled to be compatible with the GTRI tunnel test section area of $1290 \mathrm{in}^{2}$. Based on Reference 10 criteria, a physical blockage of 5 to 6 percent of tunnel area was desired, as well as reasonably high Reynolds number. Figure 6 shows that a 0.065 -scale model produces a blockage of $5.1 \%$ and Reynolds number based on trailer length of $1.90 \times 10^{6}$ at $\mathrm{V}=70 \mathrm{mph}$, or $3.90 \times 10^{6}$ at maximum tunnel speed. These factors were incorporated into the Figure 6 design, and fabricated into the model of Figure 7 by prototype shop Novatek Inc. of Smyrna, GA. Here, a number of parameters are variable, including cab/trailer gap, cab height, a removable gap fairing, trailer leading-edge and trailing-edge radii, wheels on/off, vehicle height above the road (floor) and yaw angle between the tractor and trailer. The model was mounted on a single strut, which was hollow and was later used as the blowing air supply duct into the model. This strut was mounted on a six-component floor balance below the tunnel floor, which could be yawed and raised vertically to vary ground clearance height. The test setup will be very similar to that described in Reference 3 for the blown streamlined car test program. Particle imaging laser velocimeter data was used to quantify the flowfield characteristics aft of the vehicle.

The Phase I unblown investigations were intended to determine the effects of various cab/trailer geometries prior to initiation of the blowing tests. Figures 8 and 9 are plots of drag coefficient (based 
on a model truck projected frontal area of $\mathrm{A}=0.4542 \mathrm{ft}^{2}$, including the wheel projections) versus freestream dynamic pressure, $q=1 / 2 \rho \mathrm{V}^{2}$. Reynolds number (now based on vehicle total length) and several wind speeds in mph are also shown. The uppermost curve of Figure 8 shows a cab height lower than the trailer height, and a rather large gap ( $0.824 \mathrm{x}$ width) between the cab and trailer. The flow visualization tuft seen in the gap shows significant separation and vorticity there, and the flow unsteadiness is also seen in the data-point scatter for that run. Raising the cab height to a value level with the trailer reduces the drag coefficient by $7 \%$ at $70 \mathrm{mph}$ because it shields the sharp square leading edge of the trailer. Then, filling the gap entirely ("Hi Cab, No Gap") reduces $\mathrm{C}_{\mathrm{D}}$ by another $20.1 \%$, or $25.7 \%$ from the initial configuration. Whereas this solid configuration is not actually possible because of the need for some clearance/movement between the cab and trailer, it does represent an ideal, which might nearly be achieved by flexible connections. Figure 8 also notes the significant drag reduction if the wheels are removed. This also is not a feasible configuration, but identifies the large increment which must be added back to model test data or CFD predictions for nonwheeled vehicles. Notice in all of these runs that as the regions of separated flow are reduced, so also are the $C_{D}$ values and the $C_{D}$ variations with Reynolds number, i.e., the $C_{D}$ versus $q$ curves become flatter, and the percentage drag reductions become greater. For reference, the full-scale $\mathrm{HV}$ at $70 \mathrm{mph}$ would experience a Reynolds number of $40.0 \times 10^{6}$ at sea-level standard-day conditions.

Figure 9 shows additional drag reductions due to further geometry improvement. Run 19 is the "No Gap" configuration from Figure 8, and the curve immediately above it shows a drag increase if the no-gap cab is lowered slightly to expose the square leading (LE) edge of the trailer. This is an actual condition seen on many current faired HVs, where the fairing frequently does not extend high enough to totally shield the trailer's square top LE corner. However, for the same height difference, if the square top LE of the trailer is merely rounded (here a $3 / 8$ "radius is sufficient), $\mathrm{C}_{\mathrm{D}}$ is reduced by $8.3 \%$. Then, if a $90^{\circ}$ arc with $3 / 4$ " radius is added to each of the aft edges of the trailer (this represents the unblown pneumatic trailing edge (TE), see Figure 21 of Reference 5), an additional 7.3\% drag reduction occurs due to aft flow reattachment and reduced separation. Thus, adding unblown LE and TE round corners reduces drag by $15 \%$ over the square LE/TE configuration. Significant improvement has been achieved by the pneumatic LE and TE geometries before blowing has even been applied. It should be noted that in both of these figures, and in all the following data, the HV model was situated so that the wheels were 0.06" above the tunnel floor, and the tunnel boundary layer was eliminated by tangential floor blowing (see Reference 3 ).

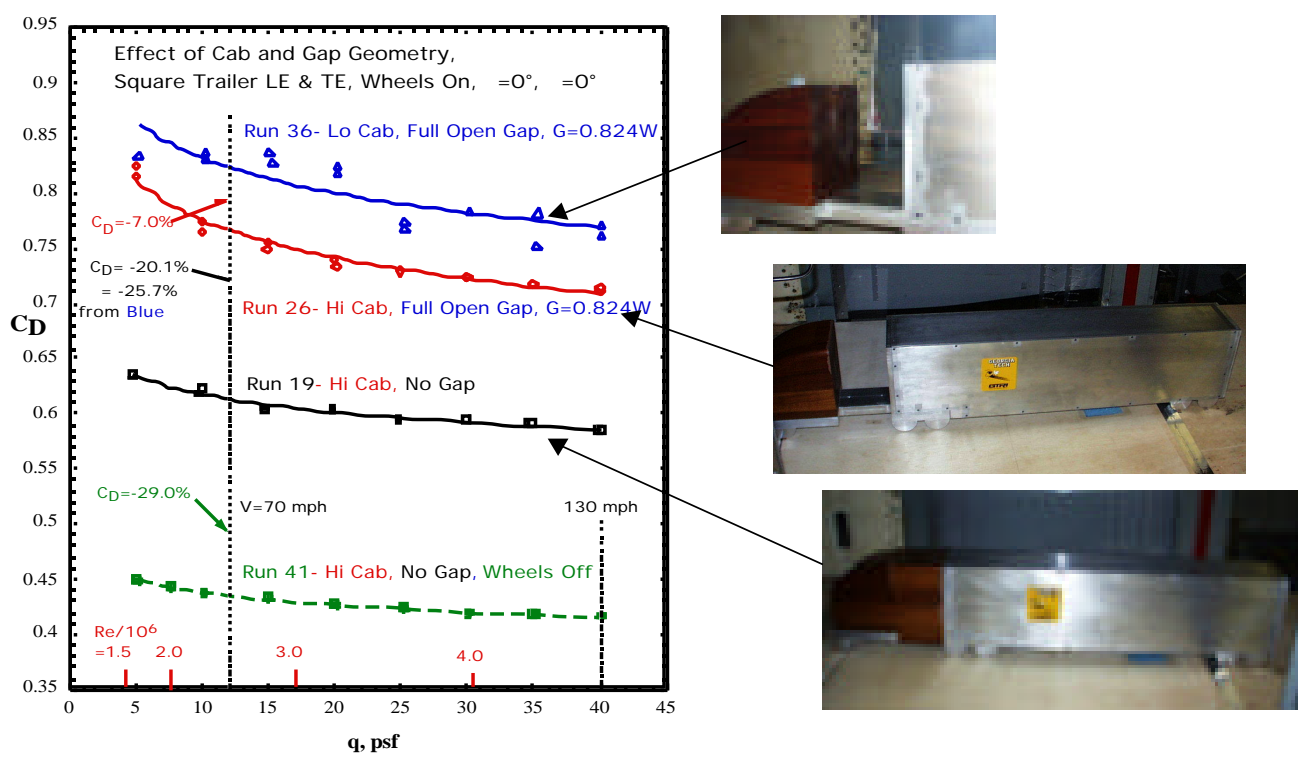

Figure 8 - Phase I Test Results for Unblown Configurations, Showing Effects of Cab Height, Cab/Trailer Gap, Reynolds Number and Wheels 


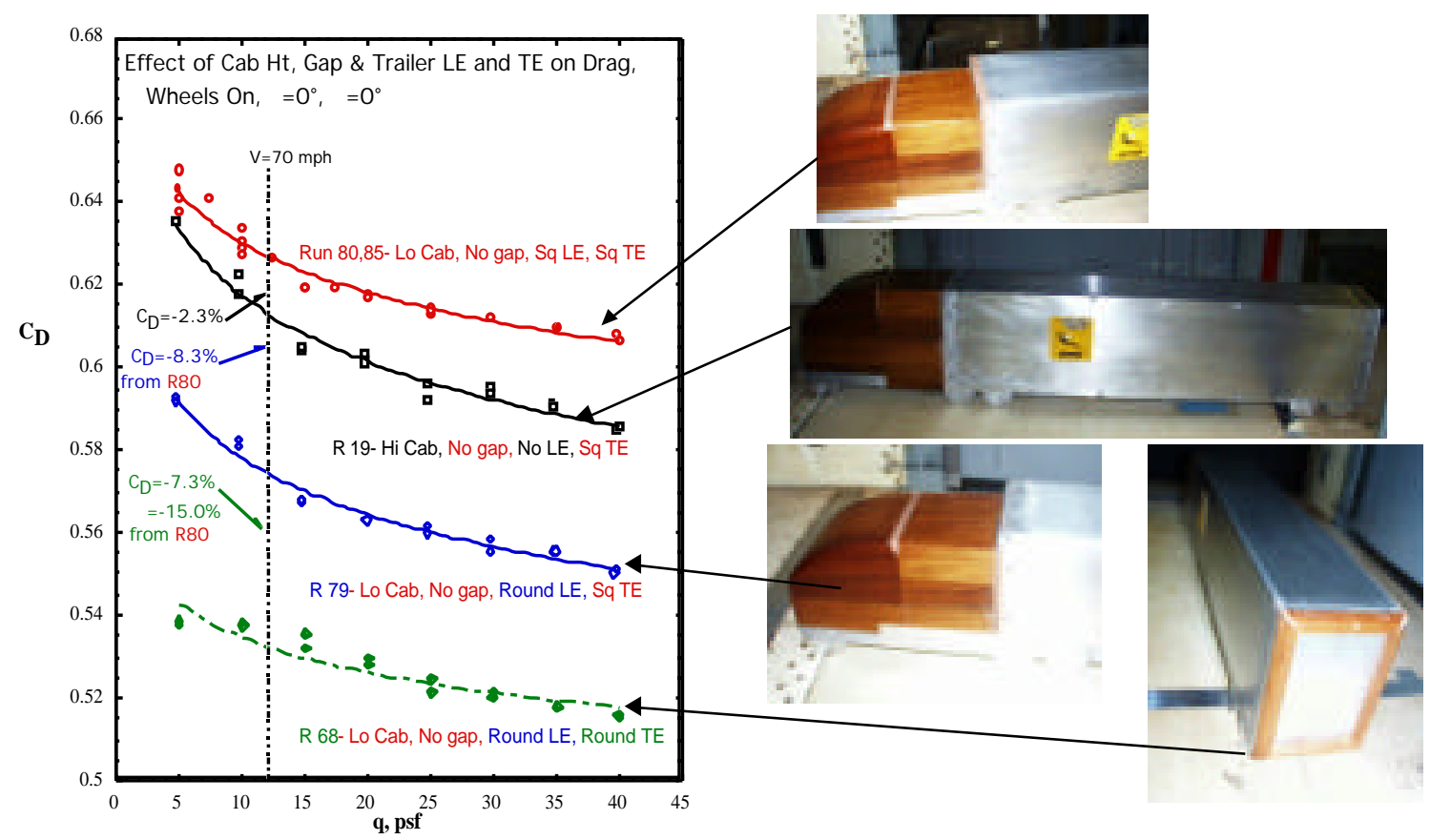

Figure 9 - Phase I Test Results for Unblown Configurations with No Gap, Showing Effects of Cab Height and Trailer Leading- and Trailing -Edge Geometries

Phase I also investigated the effects of side winds (or yaw angle) on the forces, moments and stability of the unblown HV. Figure 10 shows drag variation with side-wind angles up to $\pm 15^{\circ}$. Not only do the open-gap configurations have higher zero-yaw drag values, but they also show dramatic drag increases by a factor of 2 or more with side winds as low as only $5-7^{\circ}$ due to higher separation and greater yawed-flow over-pressures on the trailer front face. The "no-gap" models show much lower zero-yaw drag as well as lesser drag increases with yaw because these detrimental gap effects don't occur. Figure 11 shows resulting side force and yawing moment for these same configurations. Whereas there is little difference in side force $\left(\mathrm{C}_{\mathrm{Y}}\right)$ for those configurations, the models with a gap show much less yawing moment $\left(\mathrm{C}_{\mathrm{N}}\right)$ versus yaw angle and thus much less loss in directional stability $\left(\mathrm{C}_{\mathrm{N} \psi}=\mathrm{dC}_{\mathrm{N}} / \mathrm{d} \psi\right)$ than the non-gapped models. Thus the lower-drag "No-Gap" HVs may encounter a problem with directional stability as shown here.

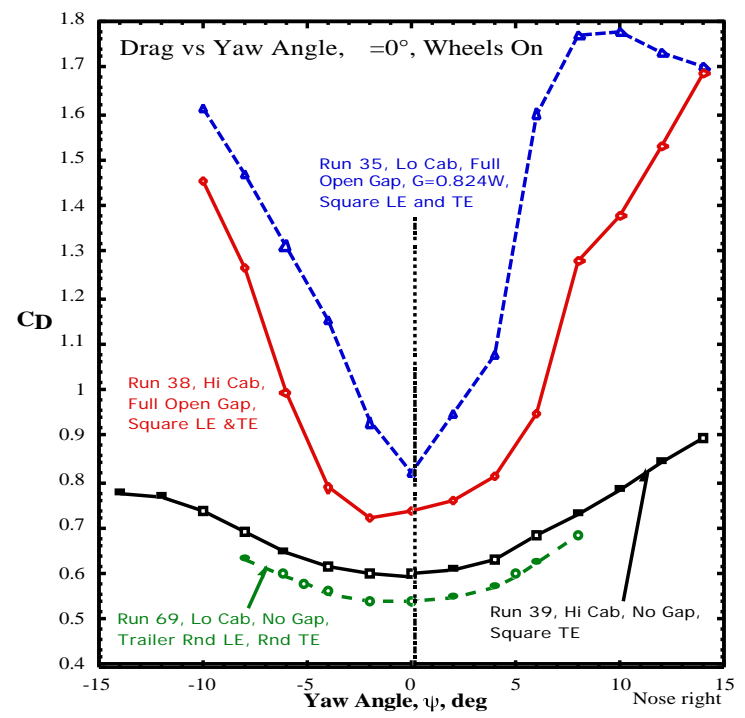

Figure 10 - Effects of Side Winds on Drag for Various Unblown HV Model Configurations 

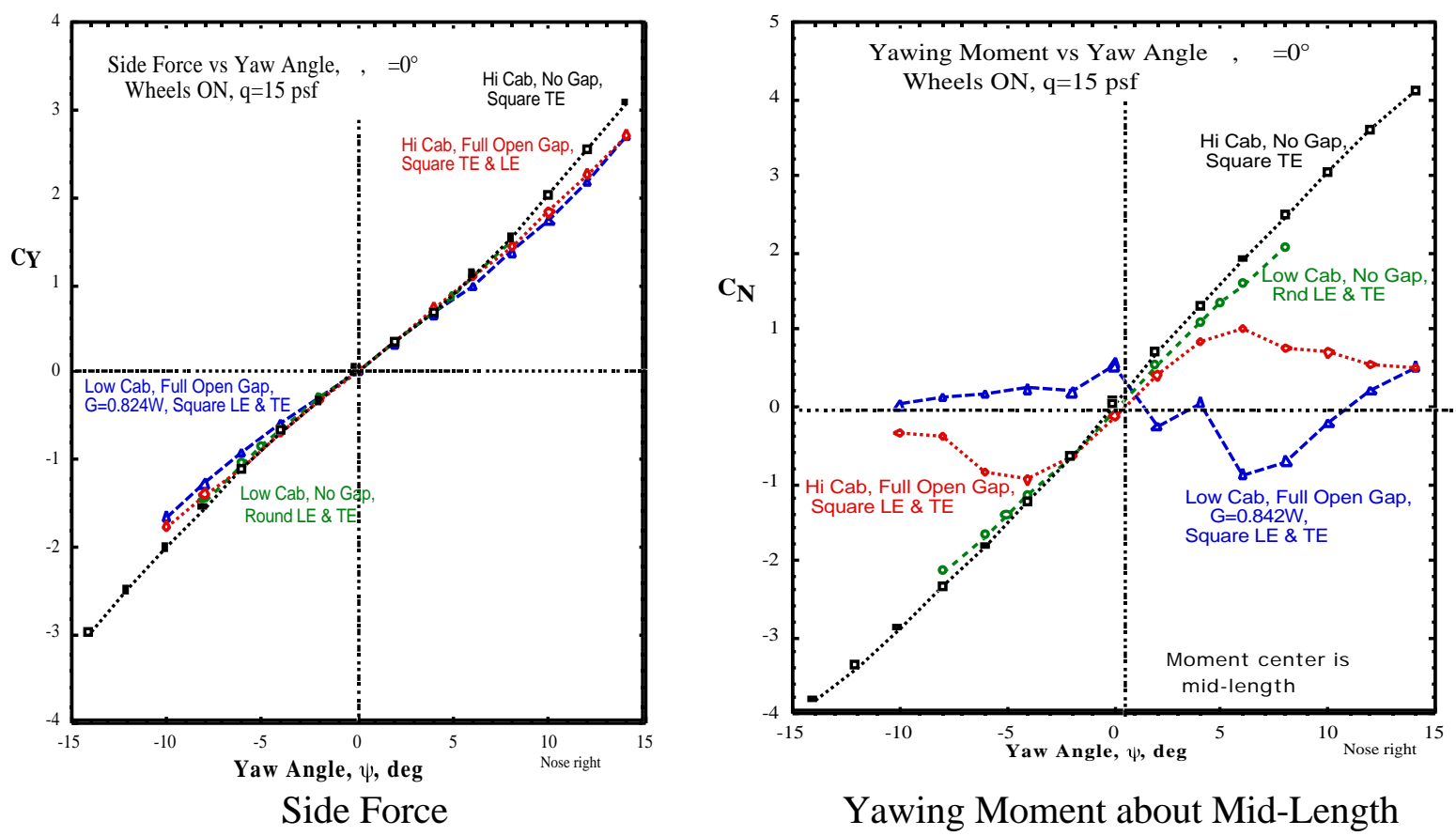

Figure 11 - Effects of Side Winds on Stability: Side force and Yawing Moment Variation (Postitive $\mathrm{dC}_{\mathrm{N}} / \mathrm{d} \psi$ is unstable)

\section{Phase II Wind-Tunnel Evaluations of Blown Heavy Vehicle Configurations}

Based on the lower-drag configurations of Phase I, Phase II was intended to thoroughly evaluate the additional aerodynamic improvements resulting from various blowing arrangements. Phase II consisted of 99 wind tunnel runs evaluating a range of pneumatic configurations and parameters, including:

- Blown trailer trailing-edge (TE) radius, jet turning angle, jet slot height, blown slot combinations and TE geometry modifications

- Blown trailer leading-edge (LE) radius and blown slot combinations

- Blowing pressure, jet velocity, mass flow, and momentum coefficient, $\mathrm{C}_{\mu}$

- Tunnel dynamic pressures from 5 to 40 psf, wind speeds from 67.3 to $190.3 \mathrm{ft} / \mathrm{s}$ (45.9 to $129.8 \mathrm{mph}$ ) and Reynolds number (based on tractor/trailer total length) from $1.61 \times 10^{6}$ to $4.61 \times 10^{6}$

- Trailer wheel configuration

- Gap between cab and trailer, plus gap side plates

- Yaw (side wind) angle

Details of these investigations are presented in the following sections and emphasize near-term aerodynamic improvements generated by these pneumatic devices. Unless otherwise noted, the 
blowing variations were run at tunnel (vehicle) wind speeds of approximately $70-71 \mathrm{mph}$ ( $\mathrm{q}=11.86$ psf) and blowing slot heights set at $\mathrm{h}=0.01$ " if not closed.

\section{Drag Reductions (Fuel Economy) or Increases (Braking \& Stability)}

The slot heights at each aft edge of the trailer could be tested either blown or unblown in any combination of the 4 , or later, with the leading edge slots on the trailer front face also blown. For best drag reduction, simultaneous blowing of all 4 of the aft trailer slots proved more effective than blowing individual slots. Flow visualization tufts in Figure 12 show jet turning of $90^{\circ}$ on all four sides, even the bottom slot blowing upwards. This is the 0.75 " radius TE configuration. Figure 13 shows even greater turning for the smaller radius $(0.375$ 'R) TE surfaces. This smaller radius on the 0.065 -scale model represents a full-sized turning radius of only 5.77'. Figure 14 shows the results of this jet turning on reducing or increasing aerodynamic drag by blowing various combinations of these aft slots. In order to represent meaningful values of the jet velocity/free stream velocity ratio, these data were run at blockage-corrected tunnel speeds of approximately 70-71 mph, dynamic pressure of $11.86 \mathrm{psf}$ and $\mathrm{Re}=2.51 \times 10^{6}$ based on tractor/trailer length. The combination of all 4 slots at the same slot height blowing together yielded the greatest drag reduction. Compared to the typical unblown baseline configuration from Phase I (full gap between cab and trailer, square trailer LE and TE, and $\mathrm{cab}$ fairing slightly lower than the trailer front) which produced $\mathrm{C}_{\mathrm{D}}=0.824$ at this Reynolds number, the blown configuration reduced drag coefficient to 0.459 at $\mathrm{C}_{\mu}=0.065$. This is a $44 \% \mathrm{C}_{\mathrm{D}}$ reduction, and the internal plenum blowing pressure required was only $0.5 \mathrm{psig}$. A second blown configuration (labeled $90^{\circ} / 30^{\circ} \mathrm{TE}$ ) used less jet turning on the upper and lower surfaces to generate even greater drag reduction: at $0.5 \mathrm{psig}, \mathrm{C}_{\mathrm{D}}$ was reduced by $47 \%$, and at $1.0 \mathrm{psig}\left(\mathrm{C}_{\mu}=0.13\right), \mathrm{C}_{\mathrm{D}}$ was reduced by $50 \%$.

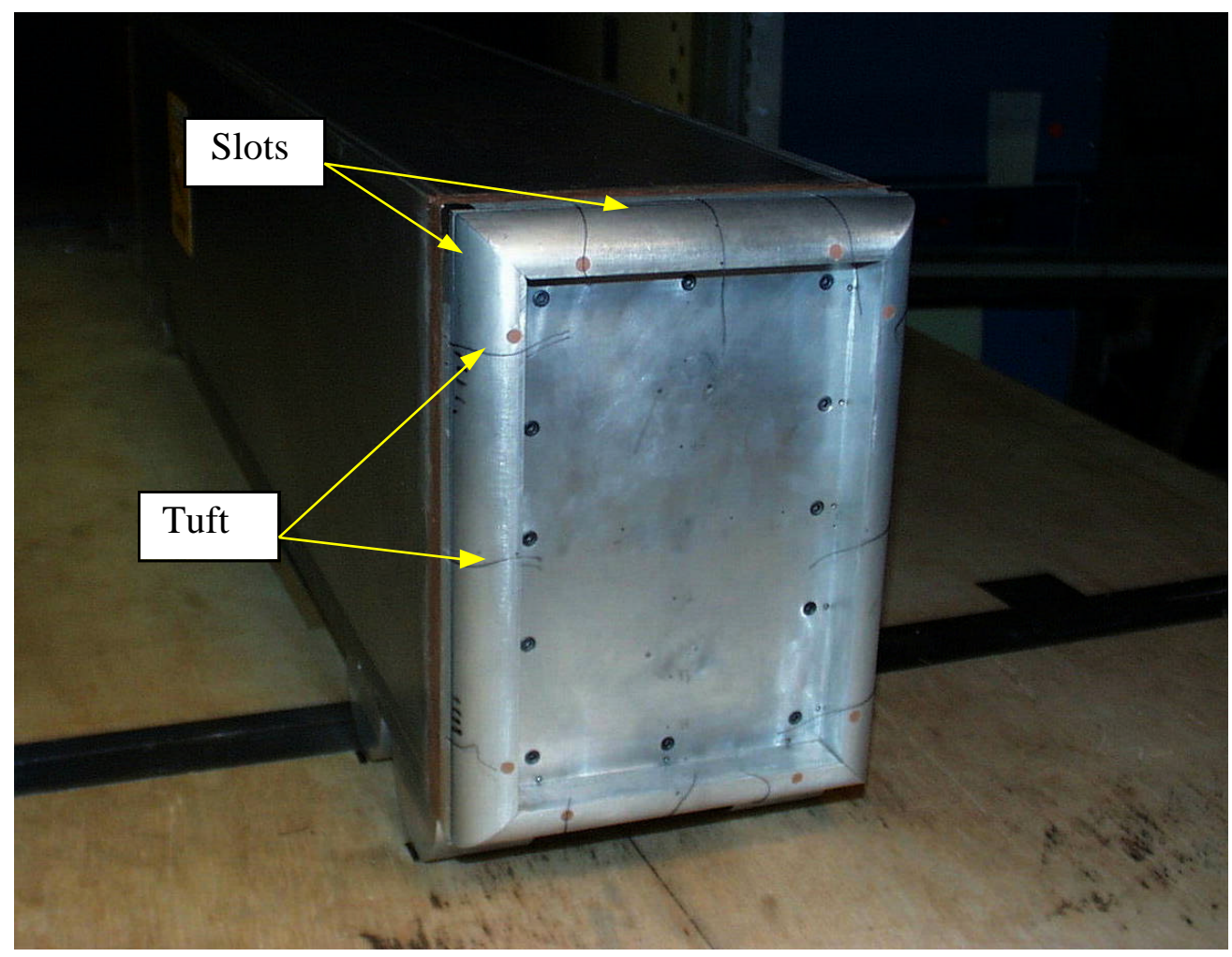

Figure 12-Jet Turning on all 4 Sides of Blown TE, 0.75"R 


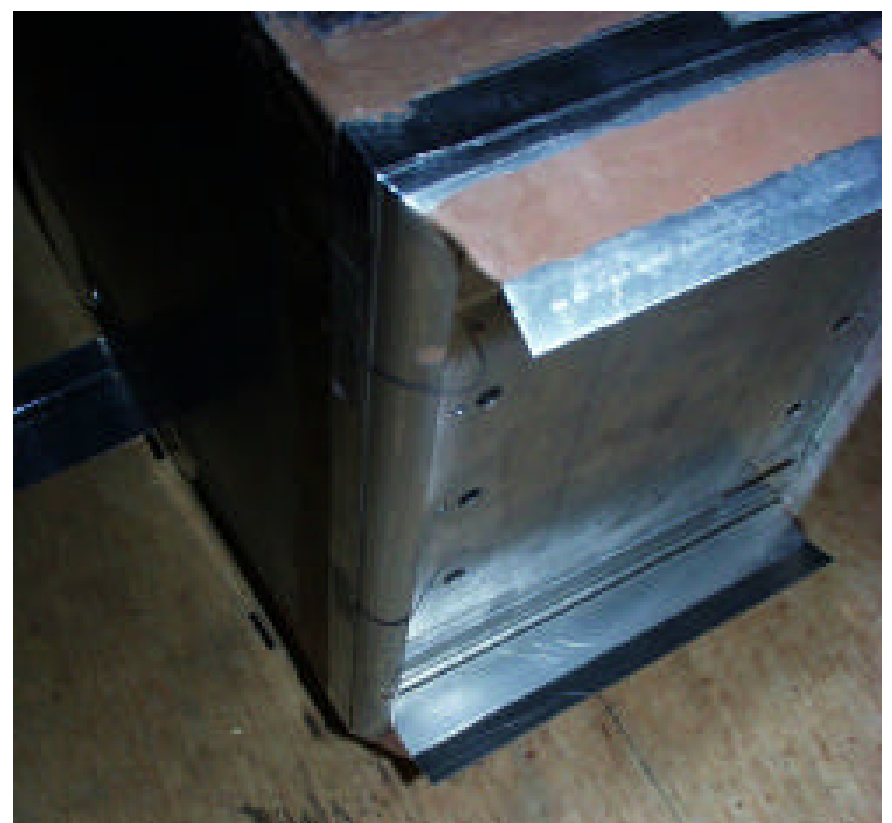

Figure 13 - Jet Turning on Smaller 0.375”R, $90^{\circ} / 30^{\circ}$ Blown Trailer TE with $1 / 2^{\prime \prime}$ plates

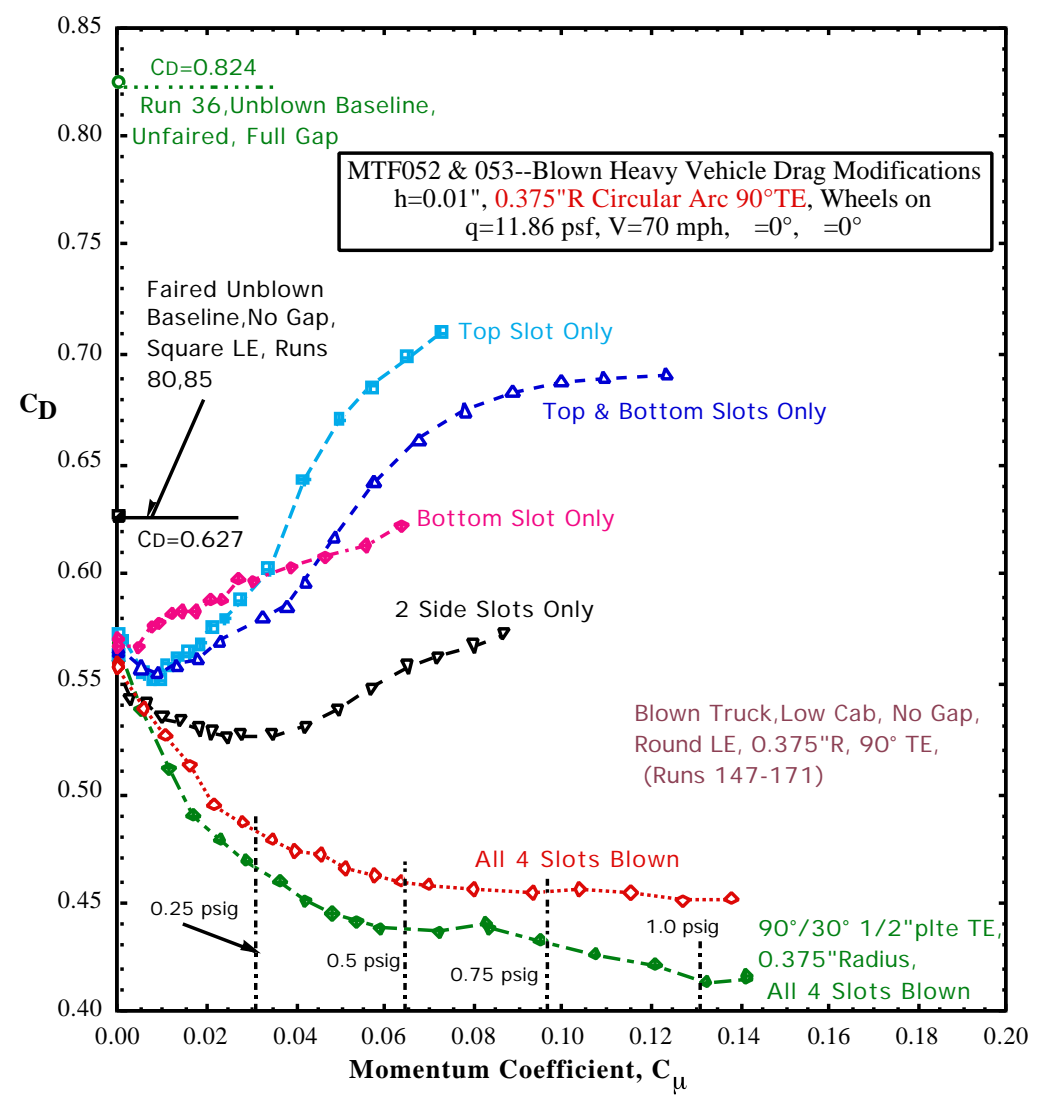

Figure 14 - Drag Reduction or Augmentation on Blown Trailer with 0.375"R Turning Surfaces

When only the top slot, the bottom slot, or both of these slots were blown in the absence of the side jets, drag initially reduced, but then significantly increased with the addition of blowing. This represents an excellent aerodynamic braking capability to supplement the hydraulic brakes. Blowing 
efficiency is plotted in Figure 15, where $\Delta C_{D}$ is an increment from the blowing-off value (negative $\Delta \mathrm{C}_{\mathrm{D}}$ is reduced drag). Absolute values of $\Delta \mathrm{C}_{\mathrm{D}} / \mathrm{C}_{\mu}$ greater than 1.0 represent greater than $100 \%$ return on the input blowing $\mathrm{C}_{\mu}=$ (total mass flow $\mathrm{x}$ jet velocity) / (tunnel dynamic pressure $\mathrm{x}$ frontal area). It is seen that the 4-slotted configuration generates values as high as -5.50 , representing $550 \%$ of the input blowing momentum recovered as drag reduction. The figure also shows the opposite trend as well, with up to $200 \%$ of the blowing momentum from top/bottom slots recovered as increased drag for braking. When $\Delta \mathrm{C}_{\mathrm{D}} / \mathrm{C}_{\mu}$ is less than \pm 1.0 , the blowing efficiency diminishes. However, should additional air be available from an onboard source such as an existing turbocharger or an electric blower, additional drag reduction is possible, as shown in Figure 16. The drag on the previous "worst unblown baseline configuration" with a large open cab/trailer gap is reduced $30 \%$ by the blown configuration. Drag coefficients of less than 0.30 are shown for faired blown HV configurations. This is in the arena of streamlined sports cars. (The drag coefficient of a 1999 Corvette coupe is $C_{D}=$ 0.29.) Figure 17 shows drag reductions as high as 590 to $600 \%$ of the input blowing momentum for the Figure 16 configurations, with the greatest reductions occurring on the previous "worst" unblown configurations due to their large regions of separated flow. Figure 18, originally intended to show that the drag curves tend to converge onto one slope independent of Reynolds number, also shows a measured drag coefficient of 0.13 for a Pneumatic Heavy Vehicle. Even though not achieved in the most efficient blowing operation range, this is an 84\% drag reduction compared to the unblown baseline configuration. Note that the tractor cab in Figure 18 has "gap plates" (or fairing extensions) instead of the full "No Gap" fairing, and is thus much closer to actual tractor/trailer configurations.

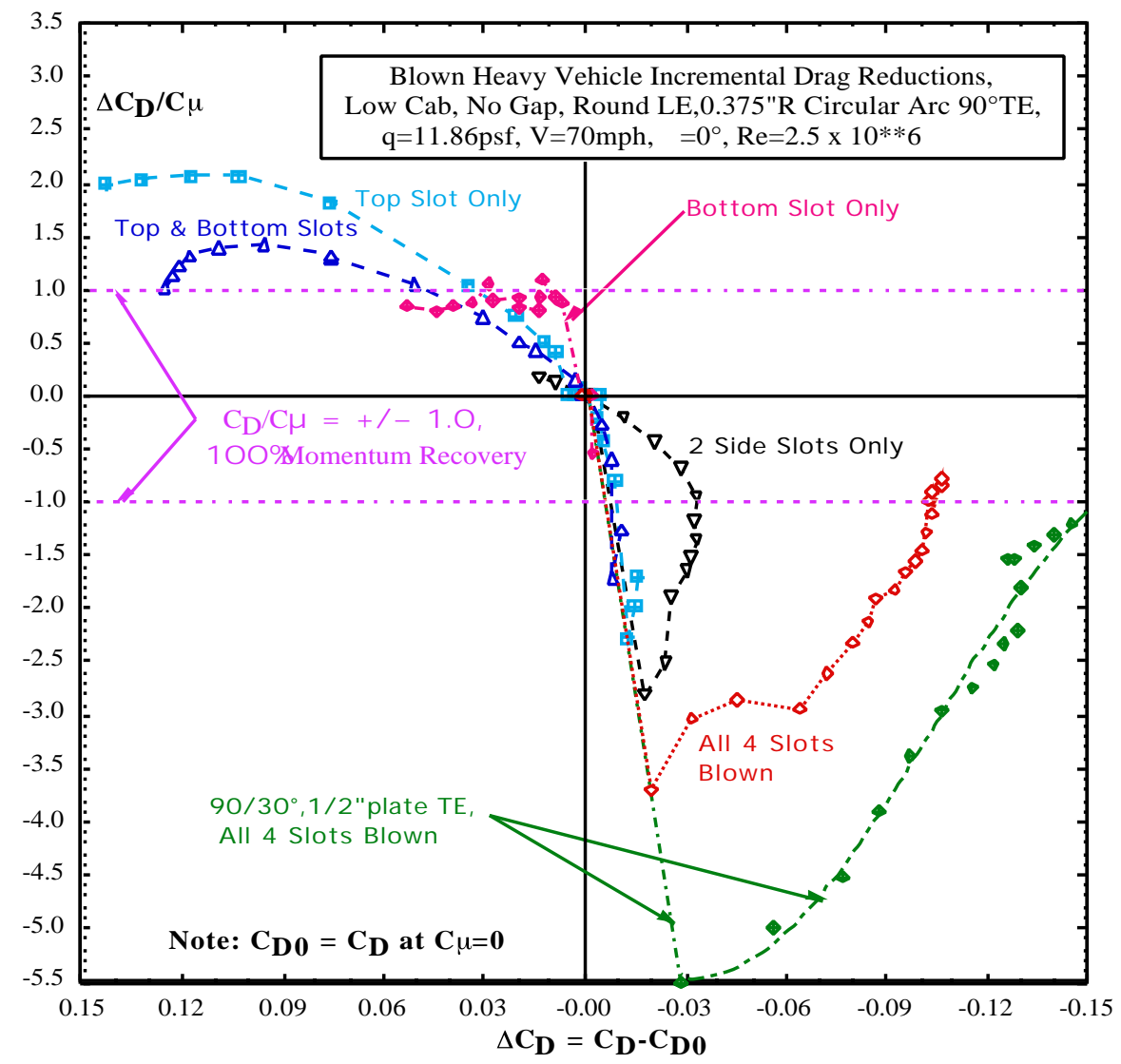

Figure 15 - Blowing Efficiency \& Drag Increments due to Blowing Slot Configuration 


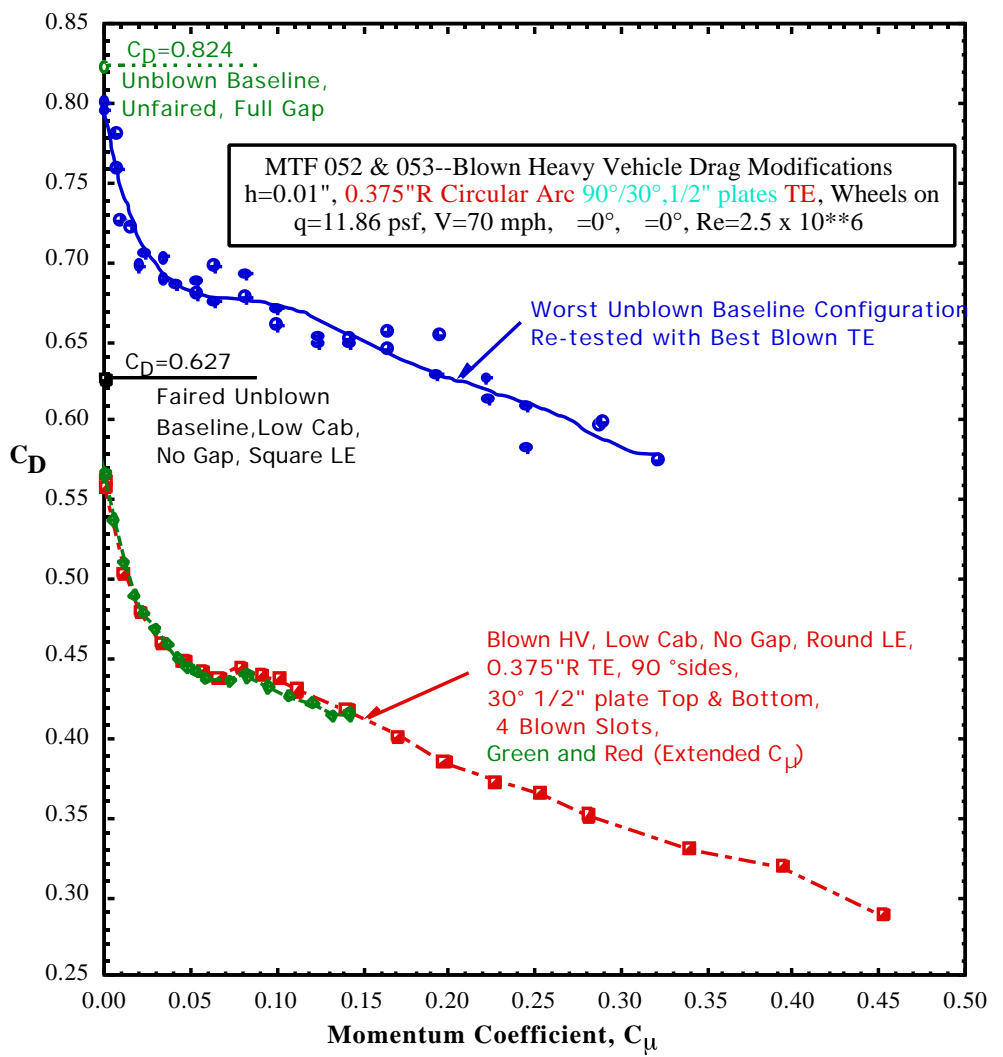

Figure 16 - Additional Drag Reductions and Comparative Configurations

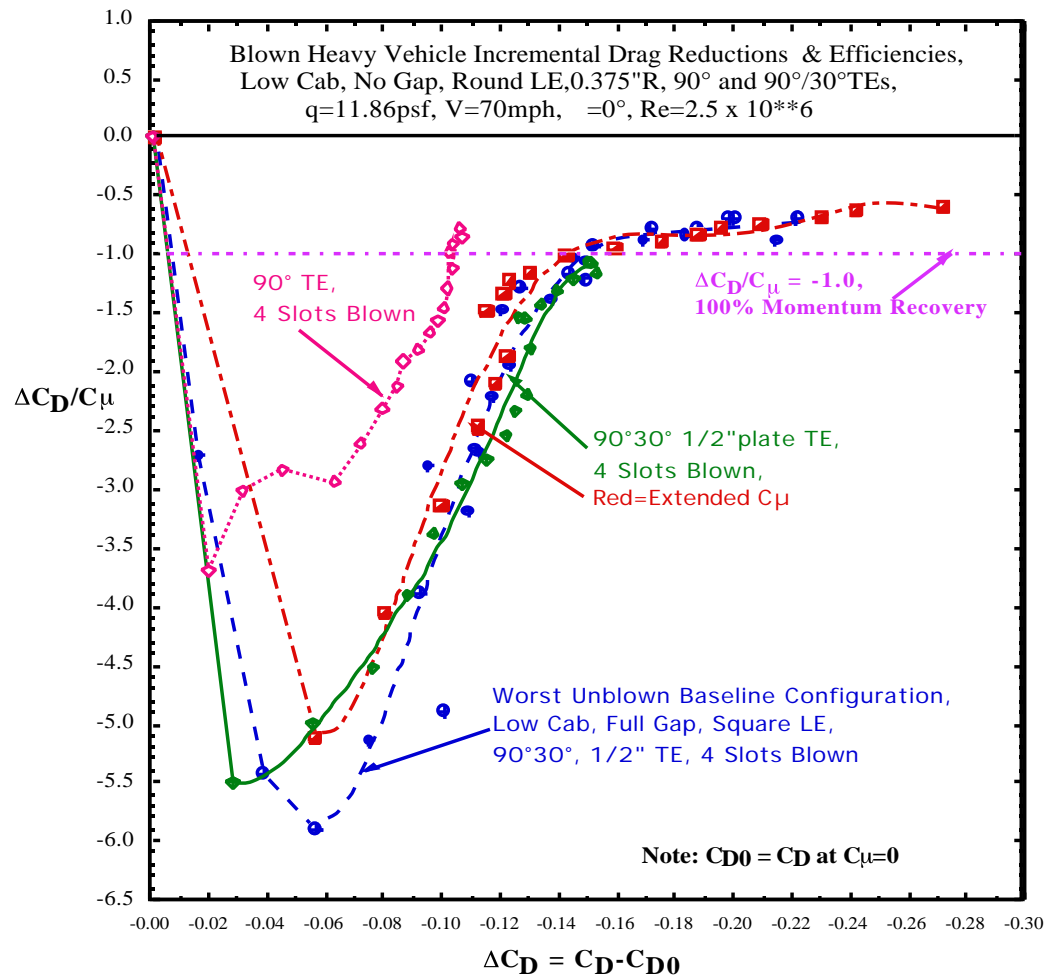

Figure 17 - Blowing Efficiencies and Drag Increments at Increased Blowing Levels 


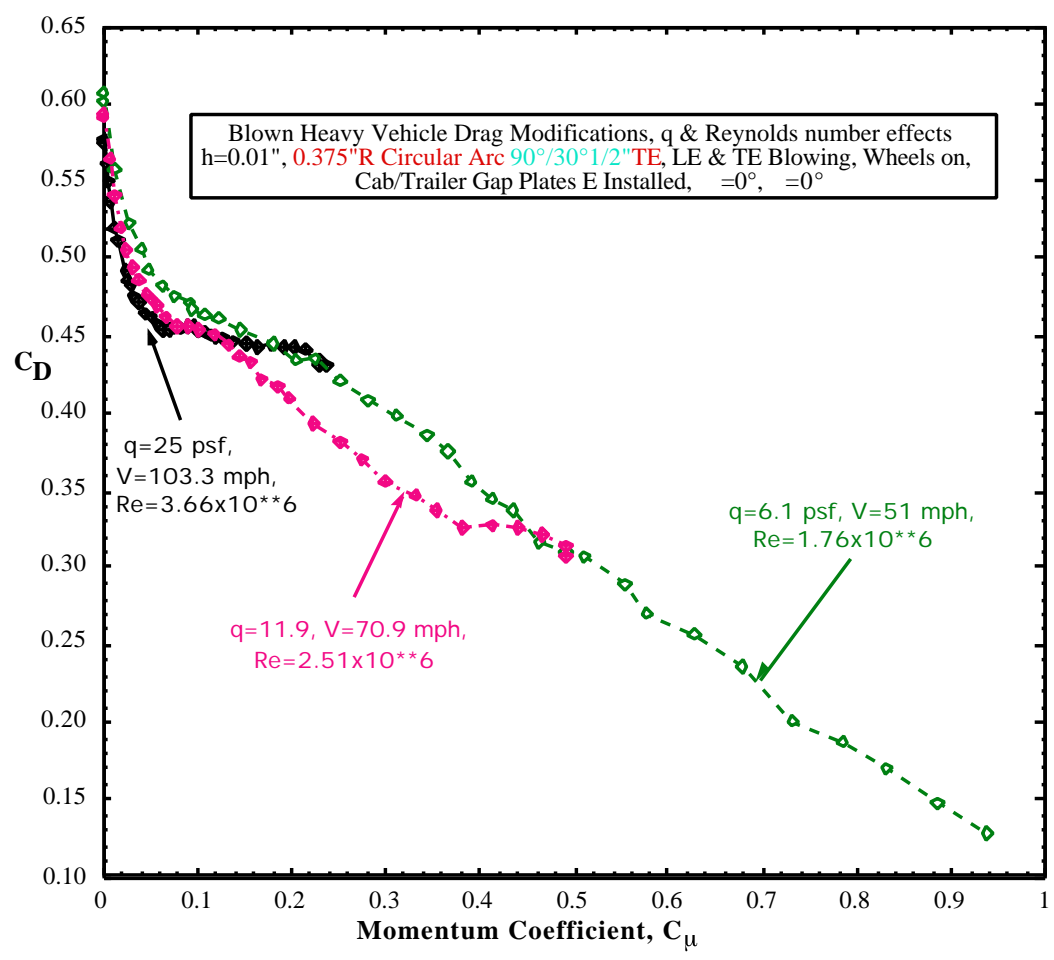

Figure 18 - Reynolds Number Effects and Increased Blowing Values, Plus Leading-Edge Blowing and Cab Gap Plates

It should again be mentioned when comparing these data to other experiments on similar GTS models being conducted by other researchers, that these GTRI data above and below include simulated wheels, which as Figure 8 shows, should add about $\Delta C_{D}=0.18$ to these non-wheeled vehicles' $C_{D}$ values, perhaps more, depending on how well the tunnel ground effects are treated experimentally. GTRI measured data are generated using test section tangential floor blowing to eliminate the floor boundary layer interference, as discussed in References 3 and 11.

\section{Lift and Down Force Generation}

Figure 19 shows lift and down force generated by various slot combinations for the blowing configurations of Figure 14. The baseline unblown configurations show slight positive lift due to underbody overpressures and cab upper surface curvature. Blowing the trailer upper slot alone can more than triple these values, which can be used to "lighten" the vehicle and thus reduce tire rolling resistance. Conversely, blowing the bottom slot can generate a down force increment 2.5 times the unblown lift, which can thus increase traction, increase braking, and reduce hydroplaning.

\section{Stability and Control}

Strong directional instability can be experienced by Heavy Vehicles at yaw angles (i.e., experiencing a side wind) because of large side forces on the flat-sided trailers (see Figure 11). Figure 20 shows the model yawed and the unblown aft pneumatic surfaces. This yaw sensitivity is confirmed by the unblown $\left(\mathrm{C}_{\mu}=0\right)$ yawing moment shown in Figure 21 , where yaw angle as small as $-8^{\circ}$ produces a large unblown yawing moment coefficient of $\mathrm{C}_{\mathrm{N}}=-2.0$ about the model mid-length. 


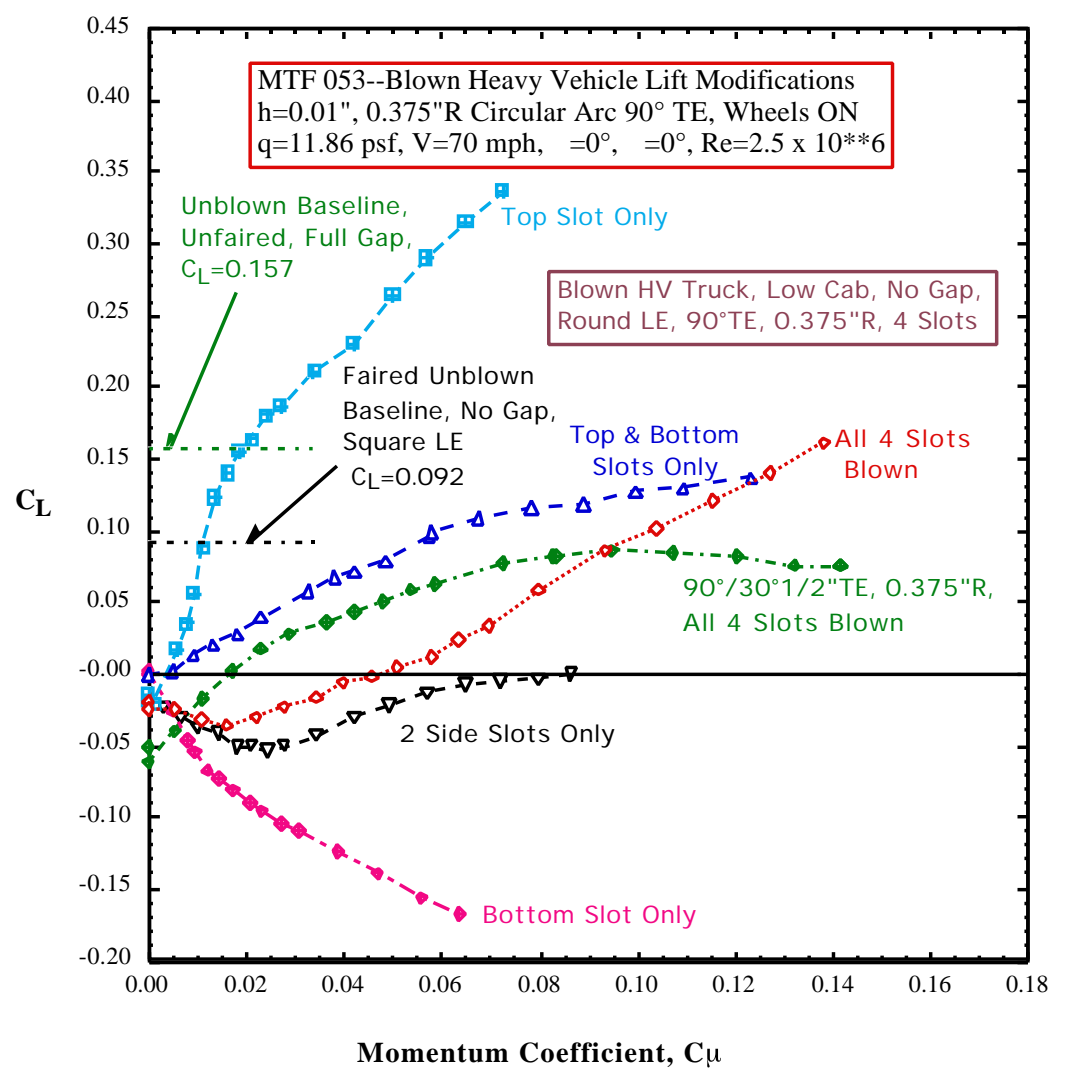

Figure 19 -Lift and Downforce Generation on Blown Trailer with 0.375"R Turning Surface

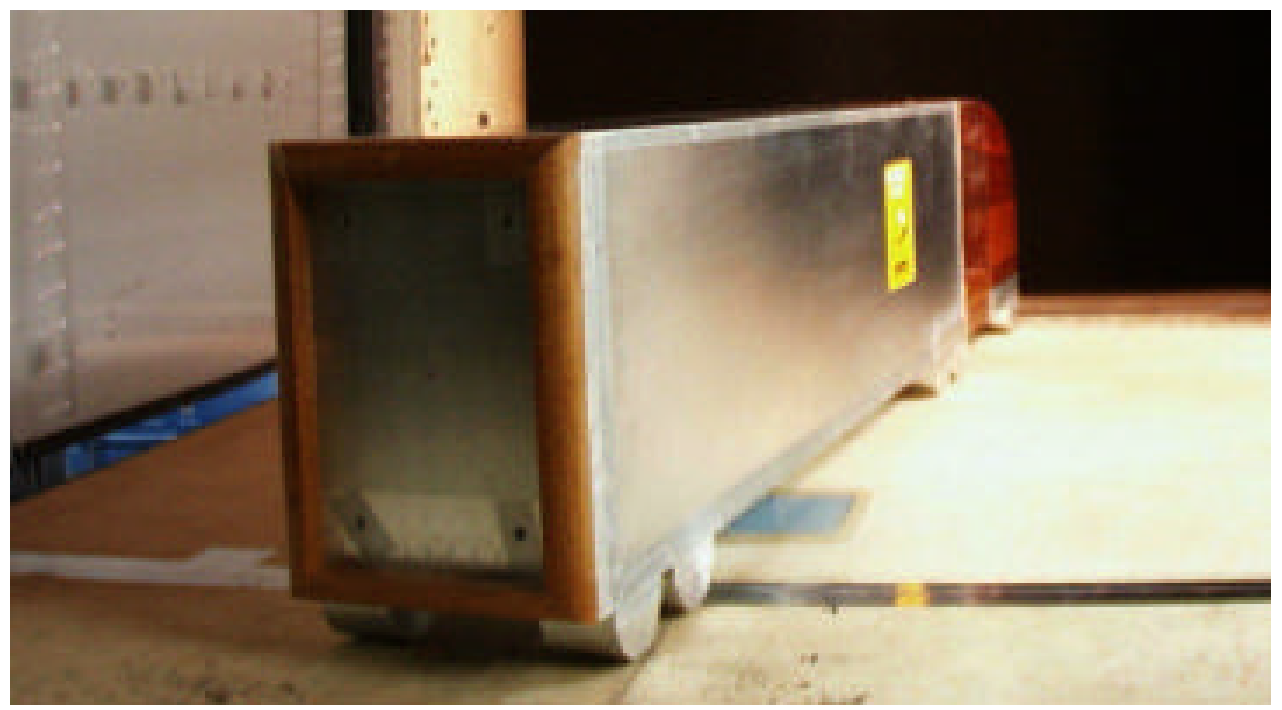

Figure 20 - Pneumatic Heavy Vehicle Model Installed Yawed (at Side-Wind Angle) in the GTRI Model Test Facility Subsonic Wind Tunnel 


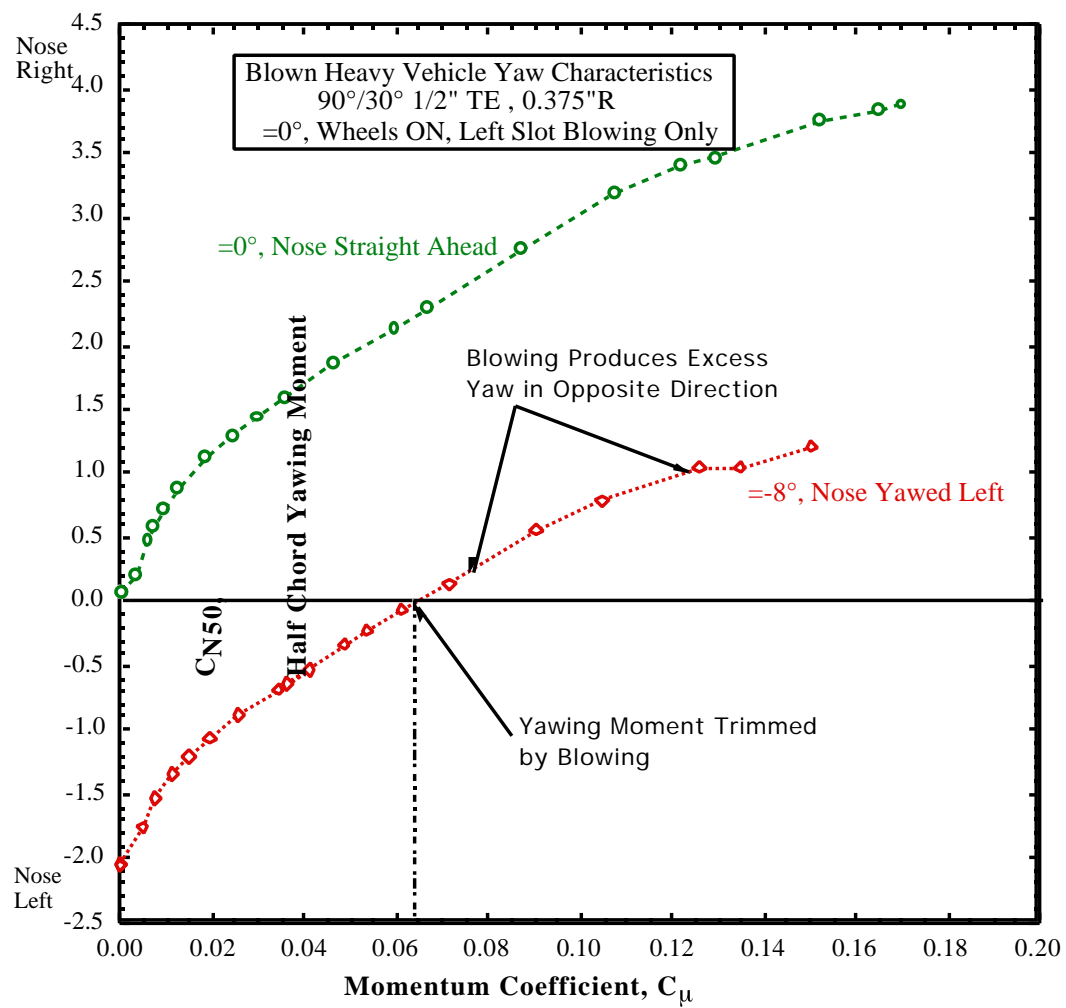

Figure 21 - Directional Control Capability Provided by Blown HV Configuration

Blowing only one side slot can easily correct this: with the nose straight ahead, blowing the left slot at $\mathrm{C}_{\mu}=0.06$ yields the equivalent opposite yawing moment $\left(\mathrm{C}_{\mathrm{N}}=+2.0\right)$. With the nose yawed left (for example, $\left.\psi=-8^{\circ}\right)$, slightly higher blowing $\left(C_{\mu}=0.065\right)$ returns this unstable yawing moment to $\mathrm{C}_{\mathrm{N}}=0.0$. Then, increasing the blowing a bit more causes the nose to yaw in the opposite direction, to the right. The opportunity for a no-moving-part quick-response aerodynamic control is apparent.

\section{Feasibility Study: Blowing Effect on Performance and Blowing Power Required}

To evaluate the effects on performance which can be produced by the above pneumatic changes in aerodynamic lift and drag, required power was calculated for a range of highway speeds. Figure 22 shows the results for a hypothetical 65,000 pound 18 -wheel tractor-trailer rig with a frontal area of 107.5 sq. ft. traveling over flat highway at sea level. Three cases are considered: a conventional rig with $\mathrm{C}_{\mathrm{D}}=0.80$ (from Figure 1, typical); a pneumatic rig showing a $35 \%$ drag reduction (i.e., the pneumatic streamlined car's drag reduction levels); and a pneumatic rig with a $50 \%$ drag reduction below the conventional rig (from Figure 14). This produces the three "Aerodynamic" horsepowerrequired curves, where drag force $\mathrm{D}=\mathrm{C}_{\mathrm{D}} \mathrm{qA}=\mathrm{C}_{\mathrm{D}}\left(0.5 \rho \mathrm{V}^{2}\right) \mathrm{A}$, and $\mathrm{HP}_{\text {req'd }}=\mathrm{DV} / 550$. Thus the required aerodynamic horsepower reduces in the same proportion as the drag coefficient, i.e., 35 or $50 \%$ at any given speed. Also included here is horsepower required to overcome rolling resistance of the tires, which is directly proportional to effective weight on the wheels times the effective tire friction coefficient, taken here to be 0.015. For the conventional rig, the HP to overcome rolling resistance varies linearly with velocity. For the blown configurations, lift varies with blowing available and dynamic pressure, so the "effective weight" of the vehicle reduces as the lift increases proportional to $\mathrm{V}^{2}$. The upper curves are the total horsepower required at the wheels (exclusive of gearing and 
internal engine losses), so total engine horsepower required would be greater. For these cases, at a sample speed of $70 \mathrm{mph}$, the horsepower required for the conventional rig to overcome drag plus rolling resistance can be reduced $24 \%$ by the lesser pneumatic configuration and $32 \%$ by the more effective one. If fuel consumption is reduced proportionally, these numbers indicate considerable increase in cruise efficiency for these blown vehicles. Note also how blowing lessens the dominance of aerodynamic drag at higher speeds. For the conventional Heavy Vehicle, horsepower required to overcome drag is equal to horsepower to overcome rolling resistance at about $66 \mathrm{mph}$, but that speed moves to about $77 \mathrm{mph}$ for the $35 \% \mathrm{C}_{\mathrm{D}}$ reduction curve, and to $86 \mathrm{mph}$ for the $50 \% \mathrm{C}_{\mathrm{D}}$ reduction.

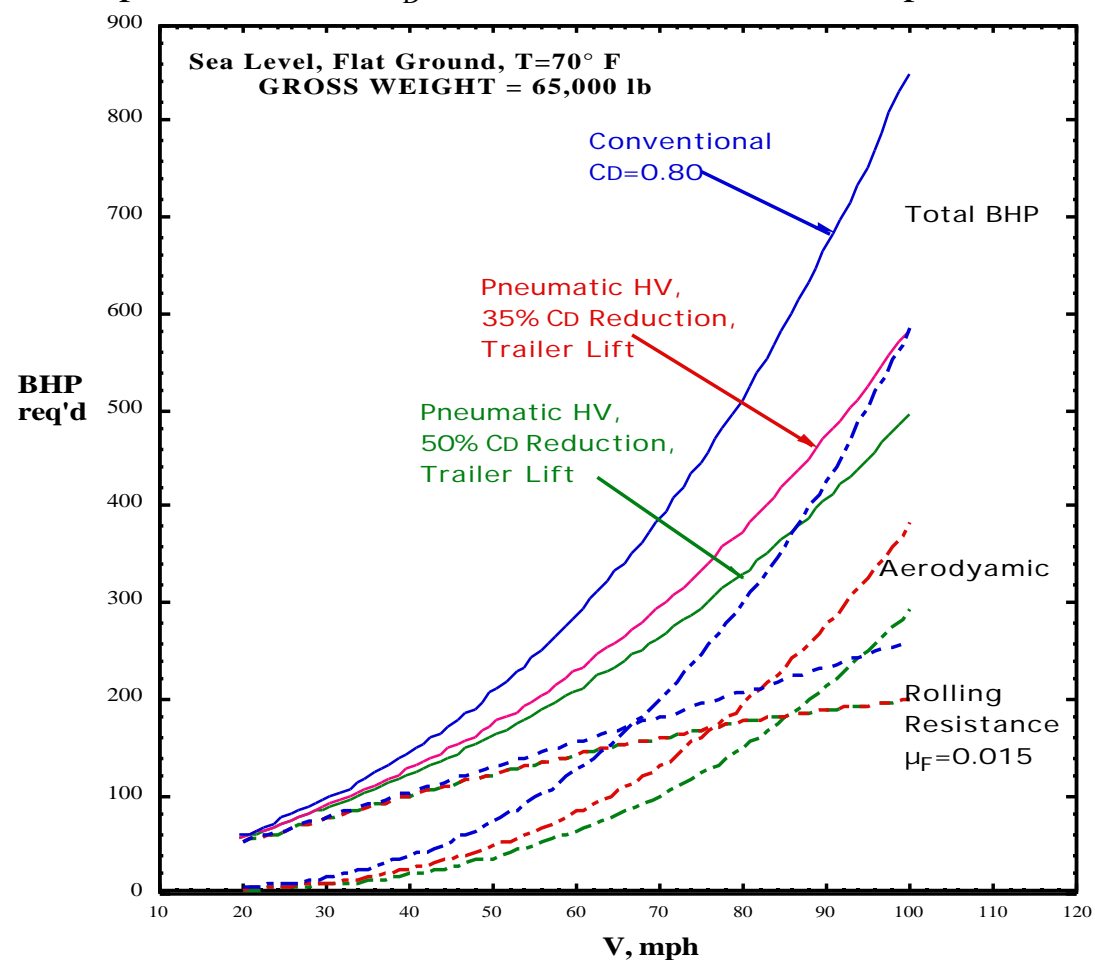

Figure 22 - Comparative Blowing-Produced Reductions in Power Required to Overcome Drag and Rolling Resistance

To the discussion above must be added a consideration of any power expended to compress the air for aerodynamic blowing. Figure 23 makes this comparison, where the blowing performance is derived from the lowest $C_{D} v_{s} C_{\mu}$ curves of Figures 14 and 16 (these are the same configurations). In Figure 23, the $C_{D}$ is converted to horsepower required at a typical speed of $70 \mathrm{mph}$ using the equations above with $\mathrm{q}=11.86 \mathrm{psf}$. This yields Curve A, $\mathrm{HP}_{\text {aero required }}$, which when subtracted from the HP value for the baseline unblown reference configuration yields Curve $\mathrm{B}$, showing $\mathrm{HP}_{\text {aero saved }}$. At this speed, the compressor HP required to compress the air from ambient to the $\mathrm{C}_{\mu}$ required is given by Curve $\mathrm{C}, \mathrm{HP}_{\text {pump }}=\Delta \mathrm{P} \mathrm{Q} / 33000$, where $\Delta \mathrm{P}$ is the pressure rise required in psf and $\mathrm{Q}$ is the volume flow rate through the slots in $\mathrm{ft}^{3} / \mathrm{min}$. Then the net $\mathrm{HP}_{\text {saved }}$ is Curve $\mathrm{D}$, which is the difference $\mathrm{HP}_{\text {aero }}$ saved $-\mathrm{HP}_{\text {pump }}$. A maximum $\mathrm{HP}_{\text {saved }}$ occurs between $\mathrm{C}_{\mu}=0.05-0.06$, and is $43 \%$ below the baseline $\mathrm{HV}$, slightly less than the $50 \% \mathrm{HP}_{\text {saved }}$ from $\mathrm{C}_{\mathrm{D}}$ reduction alone without compressor power removed. $\mathrm{HP}_{\text {saved }}$ continues to be positive until about $\mathrm{C}_{\mu}=0.355$, at which point $\mathrm{HP}_{\text {aero saved }}=\mathrm{HP}_{\text {pump }}$. Note, however, that if the blowing power were obtained totally from the output of a turbocharger waste gate at cruise speed (i.e., no extra blowing power required), then $\mathrm{HP}_{\text {pump }}=0$ and $\mathrm{HP}_{\text {saved }}$ would converge to Curve $\mathrm{B}$, which continues to increase until $\mathrm{C}_{\mu}$ reaches the limits of the turbo output. In reality, the 
saved horsepower curve would probably end up somewhere between Curves B and D depending on the turbocharger, but this still represents $\mathrm{HP}_{\text {saved }}$ of at least $43 \%$ of the baseline vehicle's aerodynamic horsepower required.

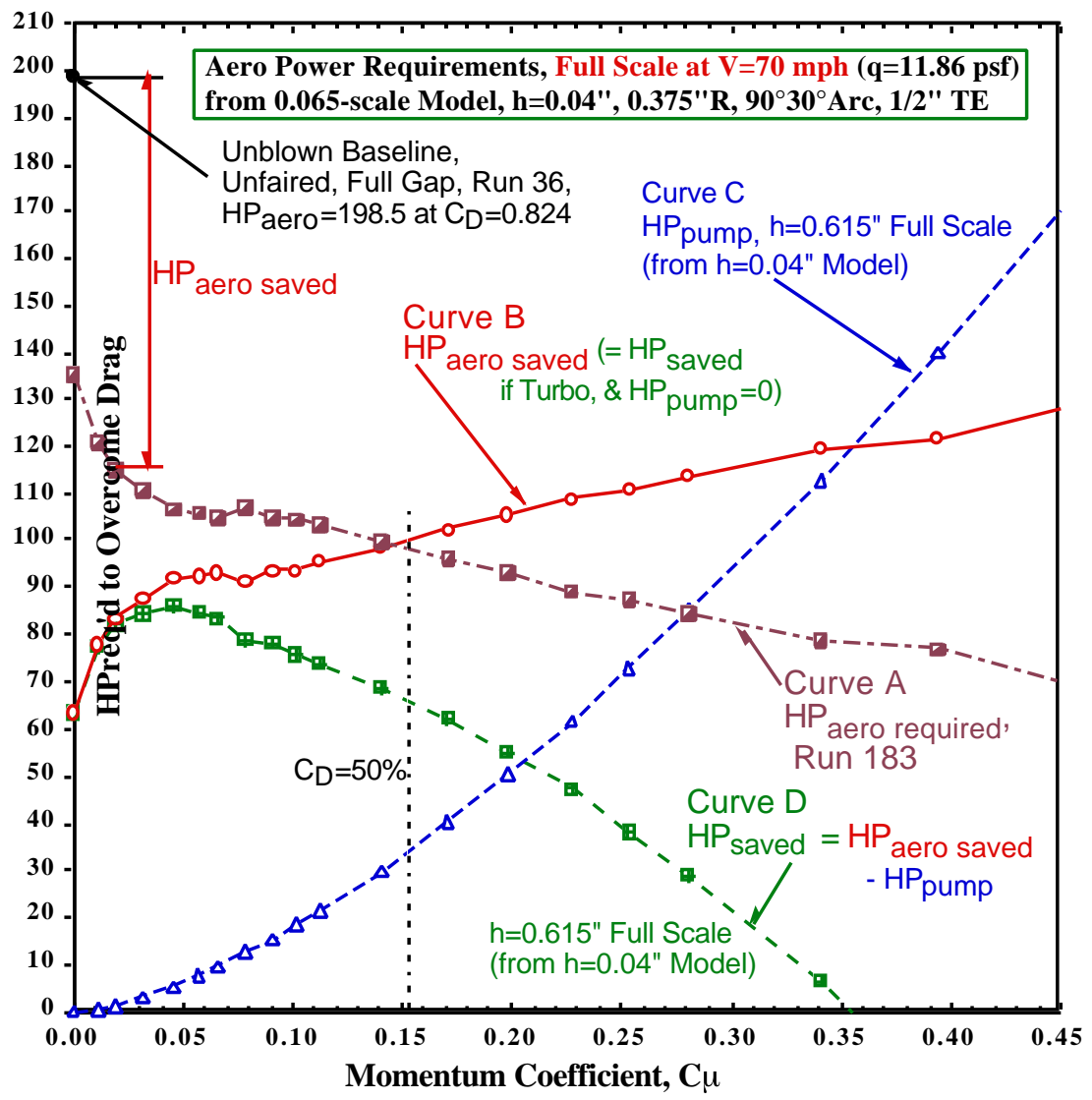

Figure 23 - Horsepower Required to Overcome Drag at 70mph, plus Required Compressor Power and Horsepower Saved

\section{ADDITIONAL APPLICATIONS OF PNEUMATIC AUTOMOTIVE AERODYNAMICS}

In addition to HV usage, the above results appear quite promising to other forms of automotive vehicles. Clearly, buses and Sports Utility Vehicles are also prone to large drag values and directional stability issues due to aft flow separation and large side panels exposed to side winds. These vehicles do offer the built-in advantage that rear corners as well as front corners are usually already at least partially rounded, and thus application of a blown system like that above would be easier than on square-edged HVs whose design doesn't want to yield internal volume in the trailer. Discussions between GTRI personnel and representatives of these industries are already underway. The possible payoffs are implied in Figure 24, which plots yearly fuel consumption in the US for various vehicle types (from References 12 and 13). Whereas automobile fuel usage is relatively level in recent and projected years, values for HVs and buses continue to rise with year, but light trucks and SUVs continue to rise at a much greater rate. Reduction in drag levels could help considerably here, especially relative to highway "cruise" fuel usage. GTRI personnel have also been contacted by motor home users, as these vehicles are likely to have squared and draggy front and rear corners causing high drag and fuel consumption. Another possible application is relative to improving aerodynamic performance of trains, not only high-speed bullet trains but also the very boxy freight trains. For these 
boxy trains, the key to large economic improvements depends on the average operating speed of these connected vehicles. In a related application, GTRI is also currently developing a patented aerodynamic heat exchanger that is based on these pneumatic principles and can further reduce the drag associated with the vertical radiator and related cooling system, while also adding favorable aerodynamic characteristics to the vehicle.

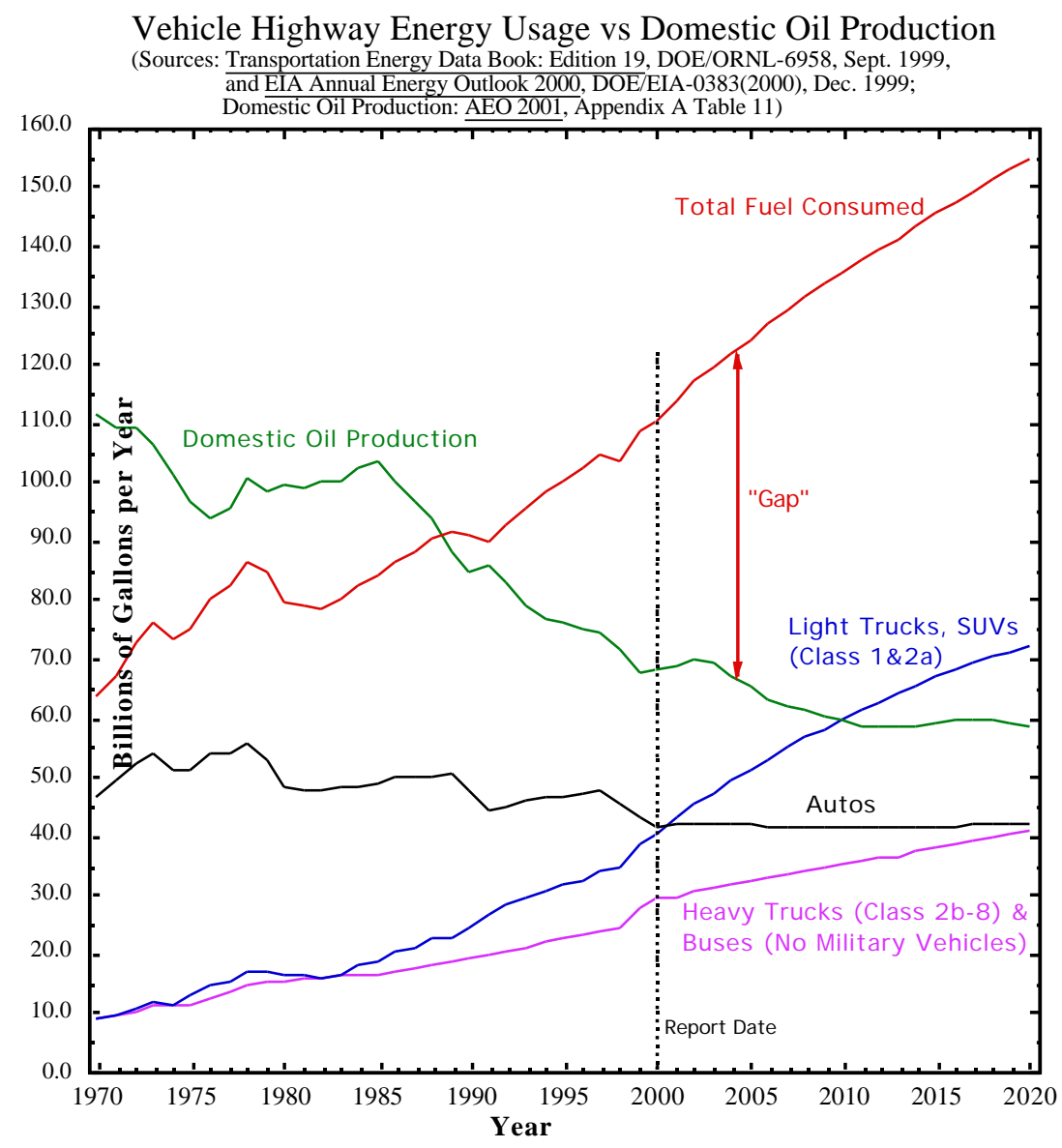

Figure 24 - Highway Energy Usage Comparisons by Vehicle Type

\section{CONCLUSIONS}

Blown and unblown wind-tunnel evaluations have been completed at GTRI in a research program conducted for the DOE Office of Heavy Vehicle Technologies to develop, evaluate, and apply pneumatic aerodynamic devices to improve the performance, economy, and safety of operation of Heavy Vehicles. The data presented above confirm the aerodynamic potential of Pneumatic Heavy Vehicle configurations. Summarizing the above:

- Drag coefficient can be pneumatically increased or decreased as desired to multiples of as high as $600 \%$ of the input blowing momentum coefficient, $\mathrm{C} \mu$.

- Drag coefficient reductions of as much as $50 \%$ were produced with internal blowing pressures of only 1.0 psig; $C_{D}$ values as low as 0.13 (an $84 \%$ drag reduction from the baseline $\mathrm{HV}$ model) were measured at increased blowing rates. 
- Variable slot combinations and blowing $\mathrm{C} \mu$ variations yielded drag decrease for fuel efficiency, or drag increase for braking and stability.

- Variable slot combinations and blowing $\mathrm{C} \mu$ variations yielded lift increases for reduced rolling resistance, or down force increases for additional traction and/or greater braking

- Blowing one side slot alone generated sufficient yawing moment to restore directional stability and offset side force due to gusts or side winds on large trailer side panels.

Thus all the original objectives for this Pneumatic Heavy Vehicle program have been experimentally confirmed: drag, lift, down force, side force and all corresponding moments can be significantly augmented (increased or decreased as needed) by blowing, and improved to the point where appreciable increases in Heavy Vehicle performance, economy, stability and safety of operation should result.

Prediction of on-the-road performance of a pneumatic Heavy Vehicle using blown drag reduction coupled with lift-enhanced reduced rolling resistance suggests that these aerodynamic improvements can result in $24 \%$ to $32 \%$ reductions in horsepower required to overcome drag plus tire rolling resistance. Even when the compressor power for blowing is factored in, savings of up to $43 \%$ are calculated in horsepower required to overcome the aerodynamic drag alone. The potential of pneumatic aerodynamic devices applied to Heavy Vehicles can be summarized as:

- Pneumatic devices on back of trailer, blowing slots on all sides and/or front top can yield dramatic improvement in aerodynamic performance, efficiency, stability, control, and safety of large commercial Heavy Vehicles

- Control of all aerodynamic forces and moments from the same pneumatic system using existing on-board air sources, which can be driver or system controlled

- Separation control and base pressure recovery = drag reduction; or base suction = drag increase

- Leading-edge (LE) suction on trailer = drag reduction

- Additional lift for rolling resistance reduction $\left(\mathrm{F}_{\mathrm{Rolling}}=\mu \mathrm{N}\right.$, where $\mathrm{N}=$ Weight - Lift $)$, or reduced lift (increased download) for traction, braking and reduced hydroplaning

- Blowing slots and their corresponding effects can be instantaneously interchanged

- Partial slot blowing or differential blowing can yield roll control \& lateral stability

- One-side blowing yields yaw control \& directional stability

- Non-moving external components = all-pneumatic systems and components with very small (if any) component drag

- Very small-size aft trailer extension = no length limitations; minimal front or top add-ons

- Splash, spray \& turbulence reduction accompanies drag reduction

- Use of existing on-board compressed air sources (exhaust, turbocharger, brake tank)

- Advanced pneumatic aerodynamic cooling systems can further reduce drag

- Fast response and augmented forces = safety of operation

- For safety, stability and/or economy, positive use can be made of aerodynamic forces/moments (lift, download, side force, yaw, roll) not currently employed in Heavy Vehicle operation, and drag can be used for braking as well as fuel efficiency 


\section{RECOMMENDATIONS}

The above aerodynamic data confirm the Pneumatic Heavy Vehicle as a viable concept for improving the aerodynamic performance, economy, stability, handling and safety of operation of large tractor trailers. Data presented has exceeded the 35\% drag reductions (previously demonstrated on streamlined cars) that the American Trucking Associations claim will result in savings of more than 1.2 billion gallons of diesel fuel per year for the US heavy trucking industry, and as much as 1.7 billion gallons per year can be saved at the 50\% drag reduction level. The following recommendations are made to suggest a meaningful continuation of this program:

- Additional wind tunnel evaluations should be conducted to even further reduce the required blowing momentum which needs to be acquired from some air source on board the tractortrailer rig. This might include slot height variation, improved blowing surface geometry, alternate jet turning characteristics, pulsed blowing, or other innovative means.

- Continued feasibility studies are needed, where the above results are transferred to the HV industry and interactions occur with tractor and trailer manufacturers, as well as with engine manufacturers, turbocharger builders, or other possible air-supply specialties.

- Preparation for a full-scale on-the-road demonstration of this technology should be begun, including further study of available air supplies and any associated penalties, plus design of a full-scale demonstrator configuration.

It is thus felt that the proof-of-concept has been successfully completed in smaller model scale, and it is recommended that a full-scale on-the-road demonstration of the Pneumatic Heavy Vehicle be undertaken.

\section{REFERENCES}

1. Hucho, Wolf-Heinrich, Editor, "Aerodynamics of Road Vehicles, from Fluid Mechanics to Vehicle Engineering," Butterworth-Heinemann, London, 1990.

2. Suski, Victor, "Improved Heavy Truck Aerodynamics, Statement of Need," American Trucking Associations, Alexandria, VA, 1995.

3. Englar, R. J., M. J. Smith, C. S. Niebur and S. D. Gregory, "Development of Pneumatic Aerodynamic Concepts for Control of Lift, Drag, and Moments plus Lateral/Directional Stability of Automotive Vehicles," SAE Paper 960673, Feb. 26-29, 1996. Also published in SAE SP-1145, "Vehicle Aerodynamics: Wind Tunnels, CFD, Aeroacoustics, and Ground Transportation Systems," pp. 27-38.

4. U. S. Department of Energy, Oak Ridge National Laboratory, "Development and Evaluation of Pneumatic Aerodynamic Devices to Improve the Performance, Economics, Stability and Safety of Heavy Vehicles", Contract No. 450000555, December 1, 1998 - December, 31, 2001.

5. Englar, Robert J., "Development of Pneumatic Aerodynamic Devices to Improve the Performance, Economy and Safety of Heavy Vehicles," SAE Paper 2000-01-2208, June 20, 2000.

6. Englar, Robert J., "Development of the A-6/Circulation Control Wing Flight Demonstrator Aircraft," DTNSRDC Report ASED-274, January 1979; and Englar, R. J,. et al, "Design of the Circulation Control Wing STOL Demonstrator Aircraft," AIAA paper No. 79-1842, August 1979. 
7. Englar, R. J. and C. A. Applegate, "Circulation Control-A Bibliography of DTNSRDC Research and Selected Outside References (Jan 1969 to Dec 1983)," David Taylor Naval Ship Research and Development Center Report 84/052, Carderock, MD, Sept., 1984.

8. Englar, R. J., "Circulation Control Aerodynamics: Blown Force and Moment Augmentation and Modification; Past, Present and Future," AIAA Paper 2000-2541, June, 2000.

9. Gutierrez, W. T., B. Hassan, R.H. Croll, and W.H. Rutledge, "Aerodynamics Overview of the Ground Transportation Systems (GTS) Project for Heavy Vehicle Drag Reduction," SAE Paper 960906, February, 1996.

10. SAE Information Report J2085, "Aerodynamic Testing of Road Vehicles; Closed-Test-Section Wind Tunnel Boundary Interference," August 1993. Also published as "Closed-Test-Section Wind Tunnel Blockage Corrections for Road Vehicles,” SP-1176, February, 1996.

11. Englar, Robert J., "Development of Pneumatic Test Techniques for Subsonic High-Lift and InGround-Effect Wind Tunnel Investigations," Paper \#18, presented at the AGARD 73rd Fluid Dynamics Symposium, Brussels, Belgium, October 4-7, 1993.

12. “Transportation Energy Data Book: Edition 19,” DOE/ORNL-6958, September, 1999.

13. "EIA Annual Energy Outlook 2000," DOE/EIA-0383(2000), December, 1999 and "AEO 2001”, Appendix A Table 11. 


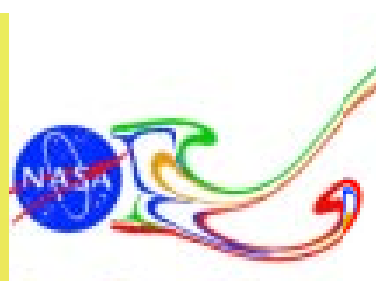

Amos Research Conter

\title{
Experimental Activities at Ames
}

\author{
Jim Ross \\ Dale Satran \\ Bruce Storms \\ JT Heineck \\ Steve Walker \\ Dave Driver
}

Heavy Vehicle Aerodynamic Drag

Working Group Meeting

$3 / 29 / 01$ 


\section{CFD Validation Testing}

- Report on 7x10 test of GTS model available

- CD-ROM of complete data set included

- Second 7x10 test started 3/19

- Planning and prep work underway for test in 12' Pressure Wind Tunnel 


\section{Current Test - Generic Truck in 7x10}

- $1 / 8$ th scale generic truck model in 7x10

- CFD validation test

- Pressure distributions

- Unsteady pressures in gap area, cab extenders, and rear door

- 3-D PIV in gap

- Oil film skin friction (limited configurations)

- Independent measurement of tractor and trailer drag

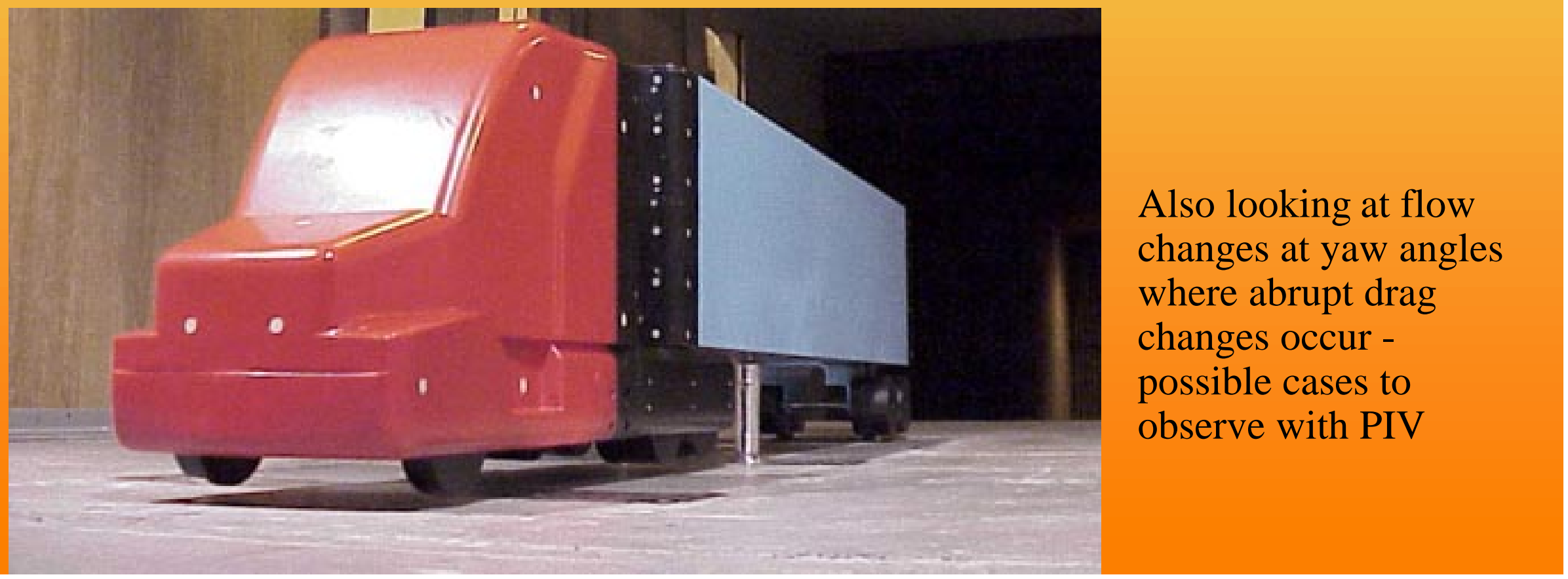




\section{Test Configurations}

- Side extender and gap variations for drag

- CDI boat-tail plates (drag delta with simple geometry change)

- Trailer vortex generators

- Underslung cargo configuration

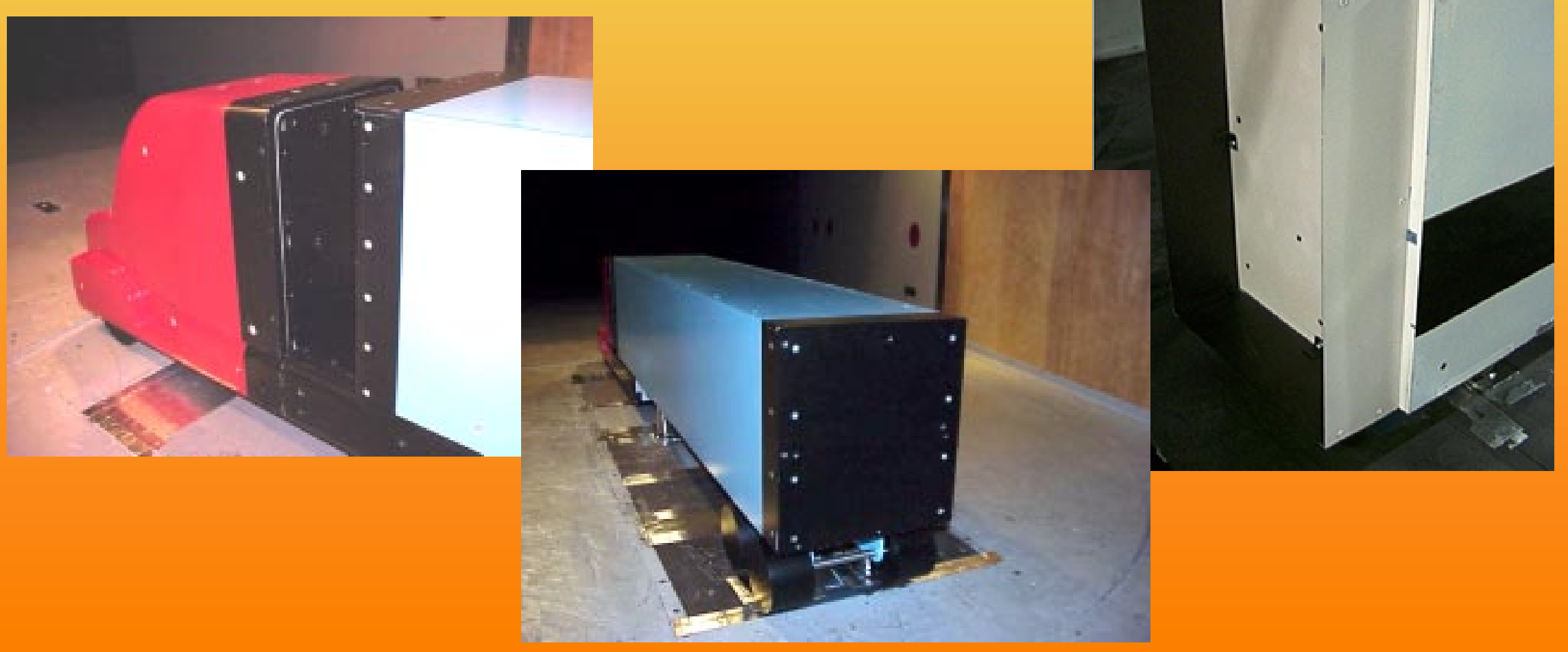




\section{Surface Pressures}

- 256 pressure taps

- No PSP - low absolute accuracy at test conditions

- Skin friction on tractor and trailer

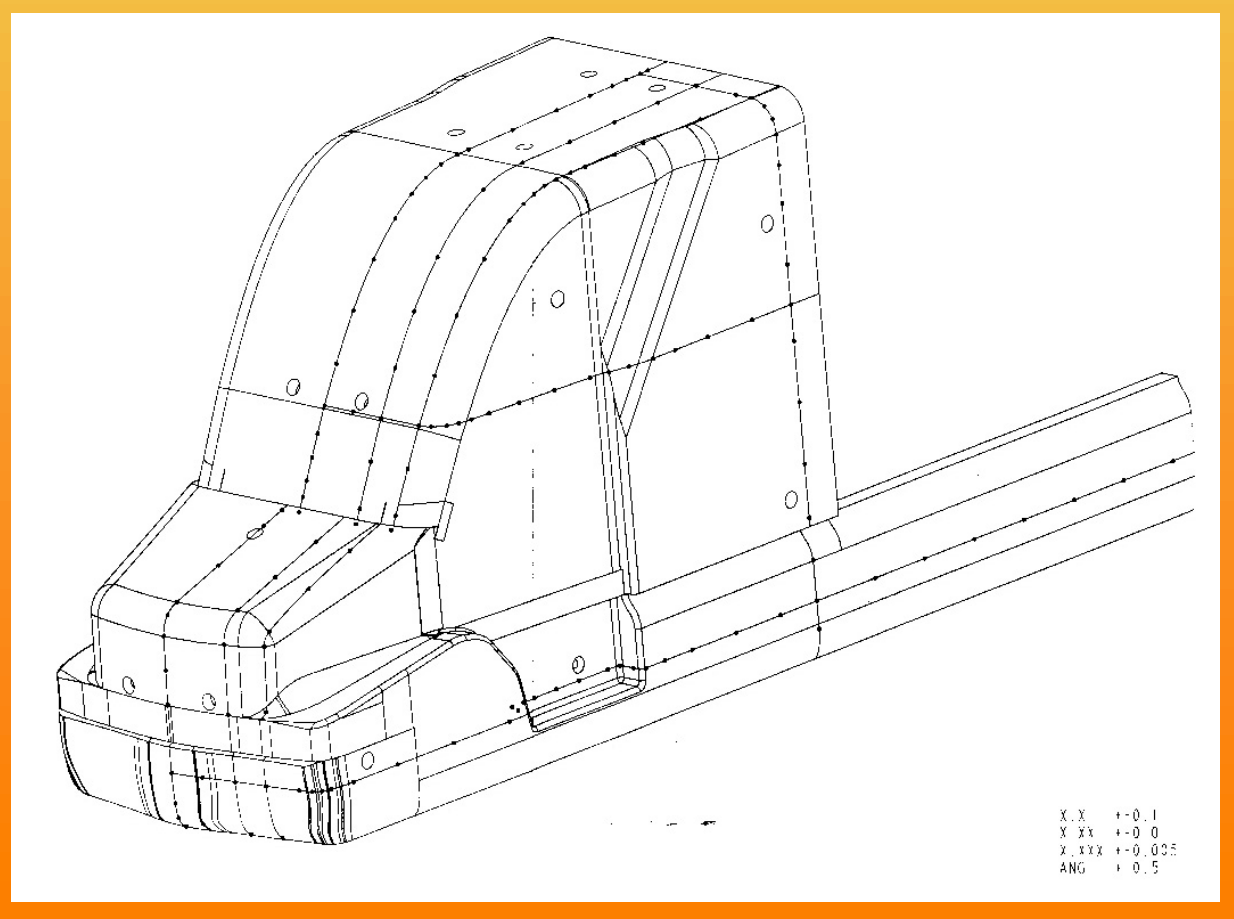




\section{Unsteady Pressure Locations}

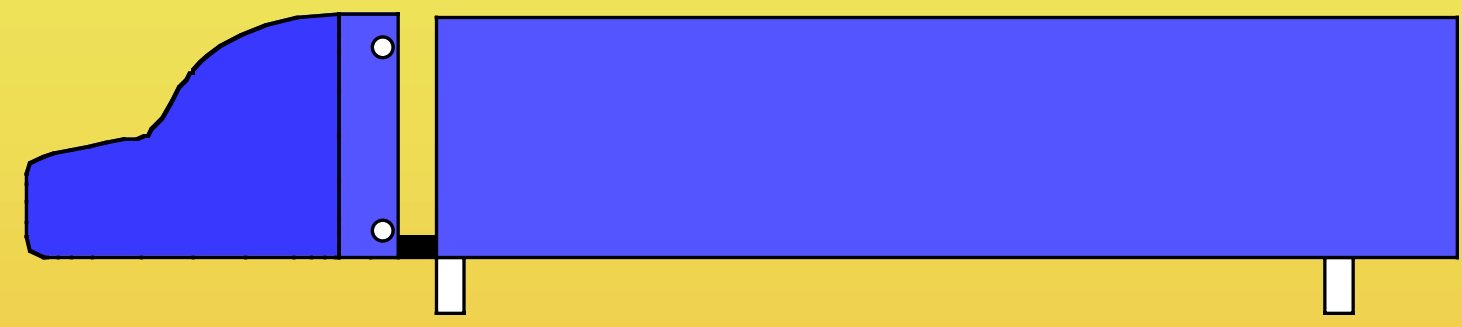

Unsteady transducers at rear of side extenders on inside surface

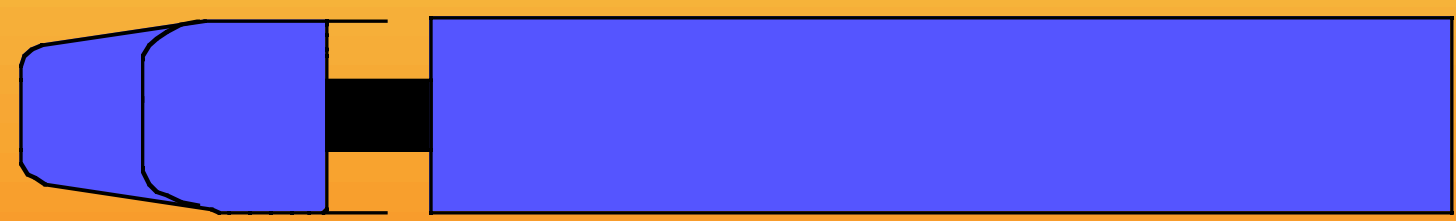

Will use Endevco 15psia transducers calibrated using piston phone

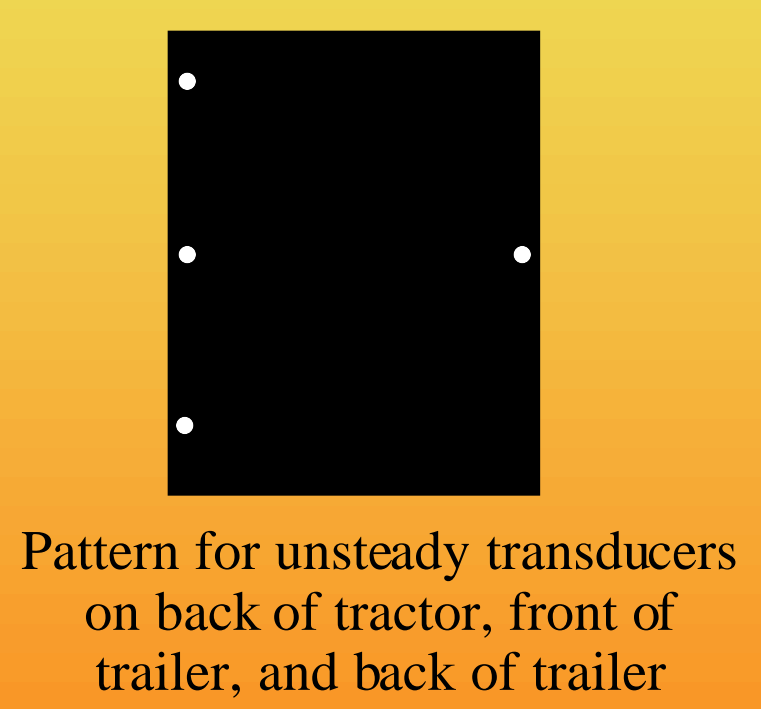

trailer, and back of trailer 


\section{PIV Surveys}

- Wake

- 4 horizontal planes

- 1 or 2 vertical cross planes

- Gap

- With and without glass side extenders

- 3 or 4 horizontal planes

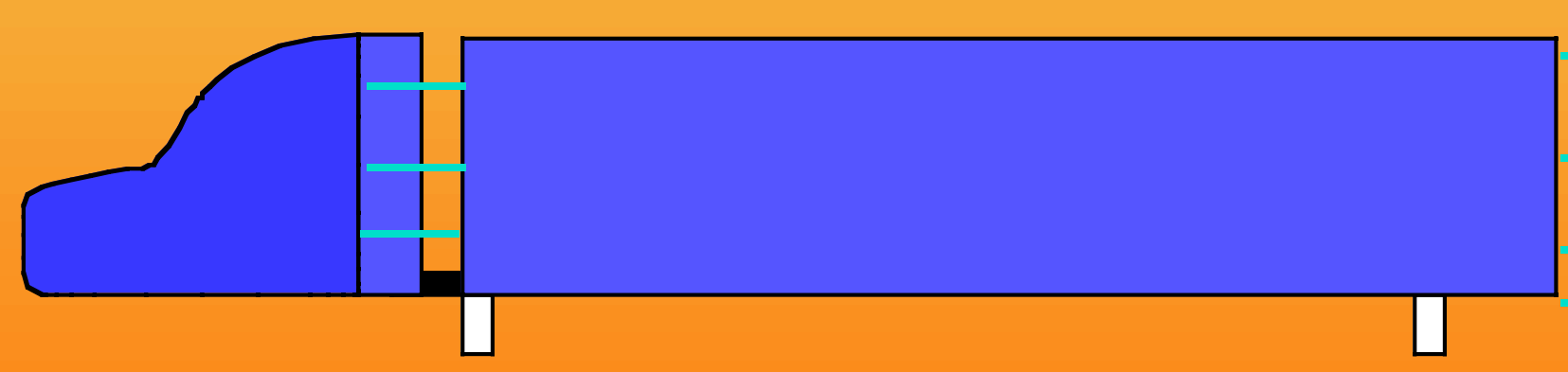




\section{Test Status}

- Aero data underway

- Tractor/trailer balance exceeding limits at high-q and yaw

- Limited to 6 deg. at $\mathrm{M}=0.27$

-14 deg at $\mathrm{M}=0.18$

- PIV hardware fab done, installation next week

- Modular mounting system will be used in 12' PWT test

- Need accurate installation measurements

- Pricing in-situ laser scan 


\section{2' Experiment Objectives}

- Generic tractor model

- Re effect on wind-averaged drag

- Range from 0.5 to 5 million (based on width) at highway speed

- Re effect on drag components - gap/side extenders, mirrors, base-drag-reduction devices, etc. and associated flow physics

- CFD validation data

- Skin friction

- Pressure distributions

»PSP \& electronic

- Unsteady pressures

- PIV

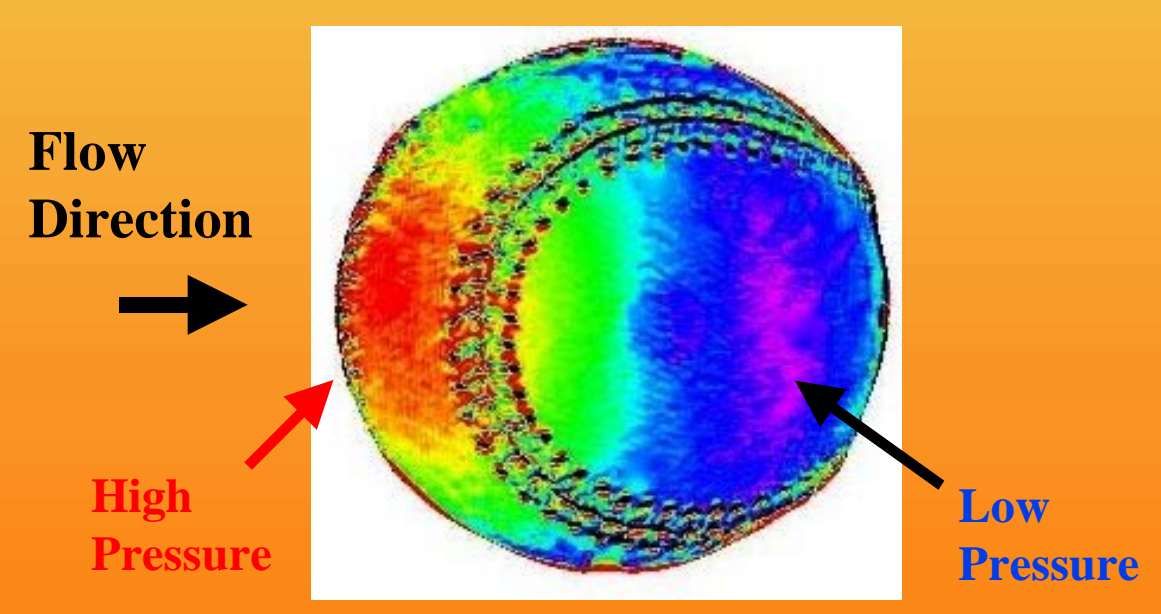

Pressure Sensitive Paint results on baseball at $120 \mathrm{mph}$ at $1 \mathrm{~atm}$. Much better at higher total pressure 


\title{
USC Experiments
}

Inter-Wind Tunnel Validation

Cab-Trailer Forces and Moments

Cab-Trailer Gap Flow Field

Base Flaps with Oscillatory Perturbations

\author{
Mustapha Hammache, Staff \\ Al Knight, Graduate Student \\ Participating Staff: \\ Fred Browand, Staff \\ Mark Michaelian, Staff \\ Phil Kassouf, Undergraduate \\ David Lazzara, Undergraduate
}




\section{Cab-Trailer Forces and Moments}

6-Components, 3 Forces, 3 Moments, Separately on Cab and Trailer

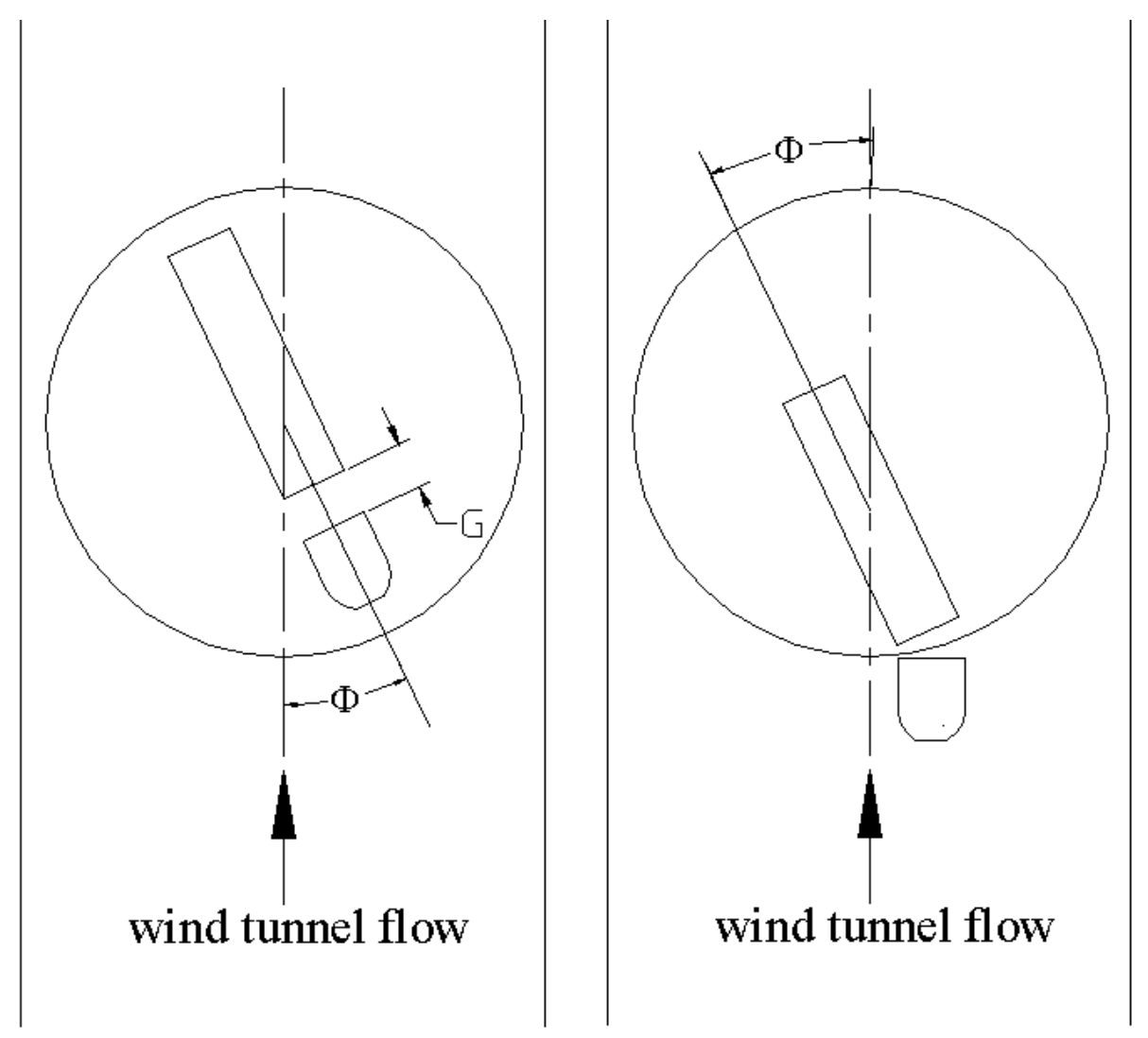


Aerodynamic Cab:Boundary Layer Trip, Wheels

Trailer : Rounded Verticals, Wheels

Aerodynamic Cab: No Trip

Trailer: Square Verticals

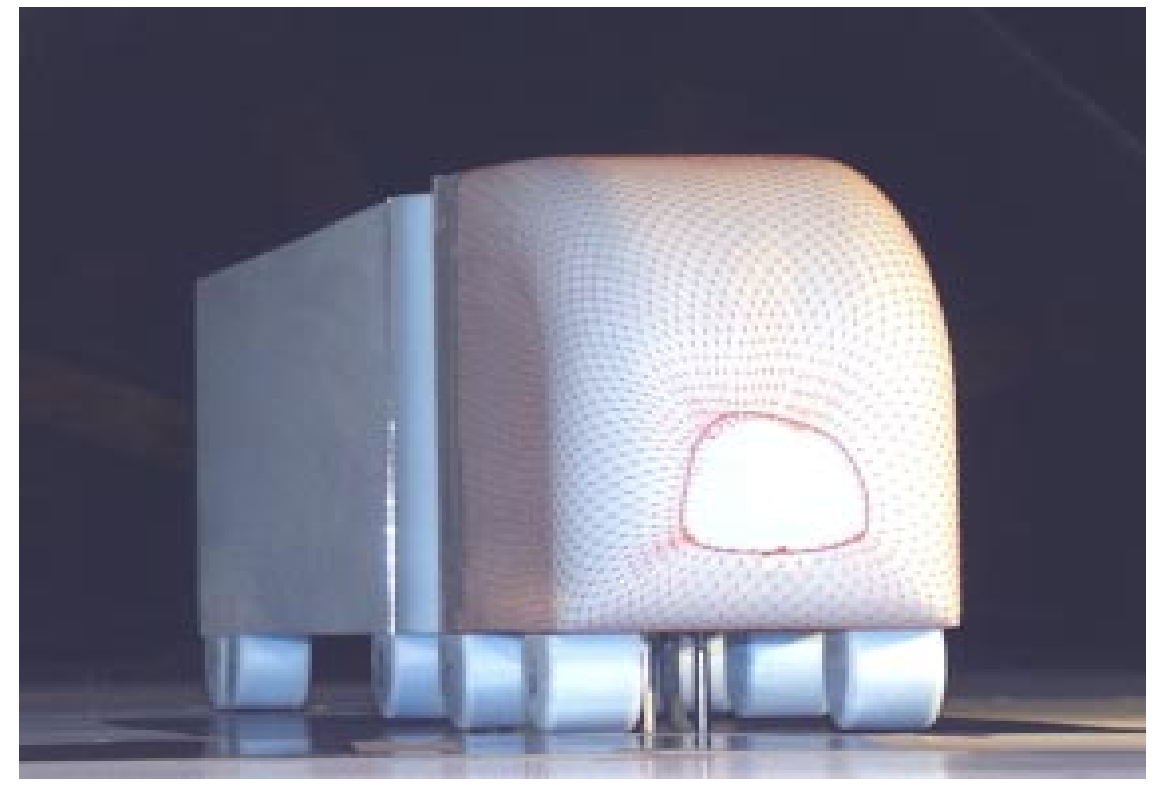

Aerodynamic Cab: No Trip

Trailer: Rounded Verticals

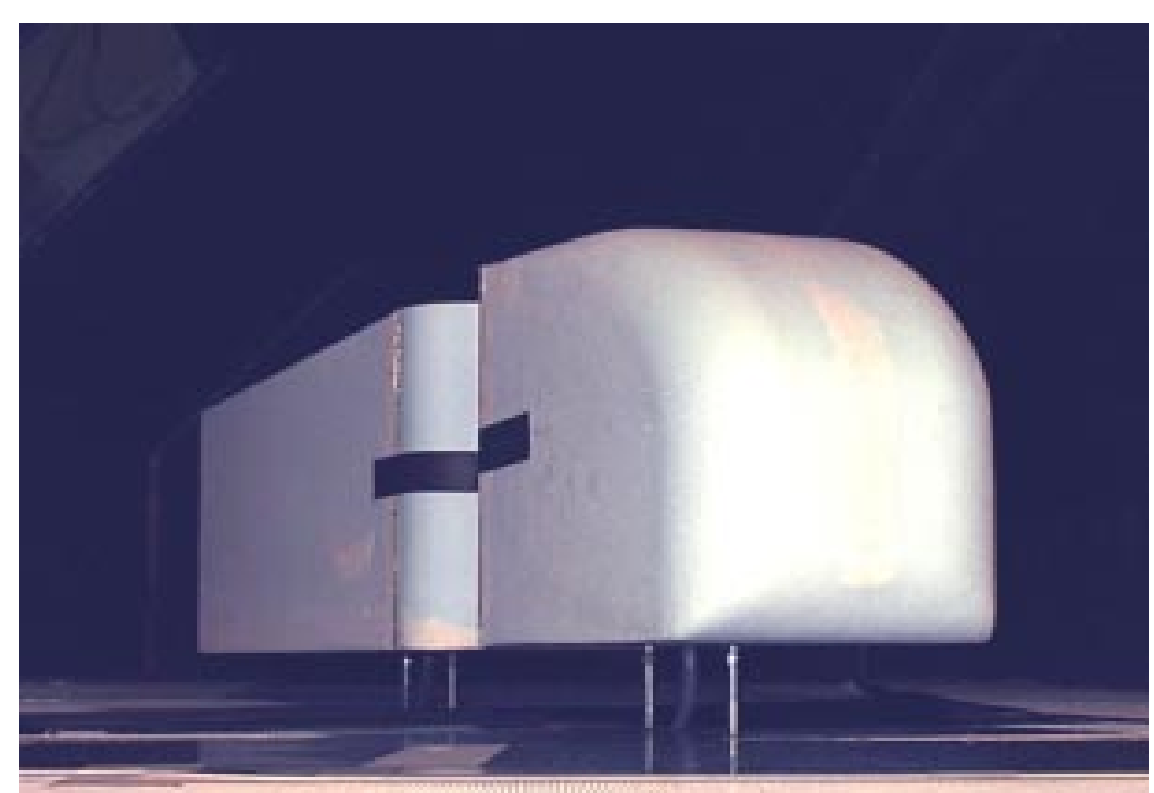


Force Coefficients as a function of Gap and Yaw Angle

Average Force (8-second average), and RMS Fluctuating Drag Force, Side Force, Yaw Moment

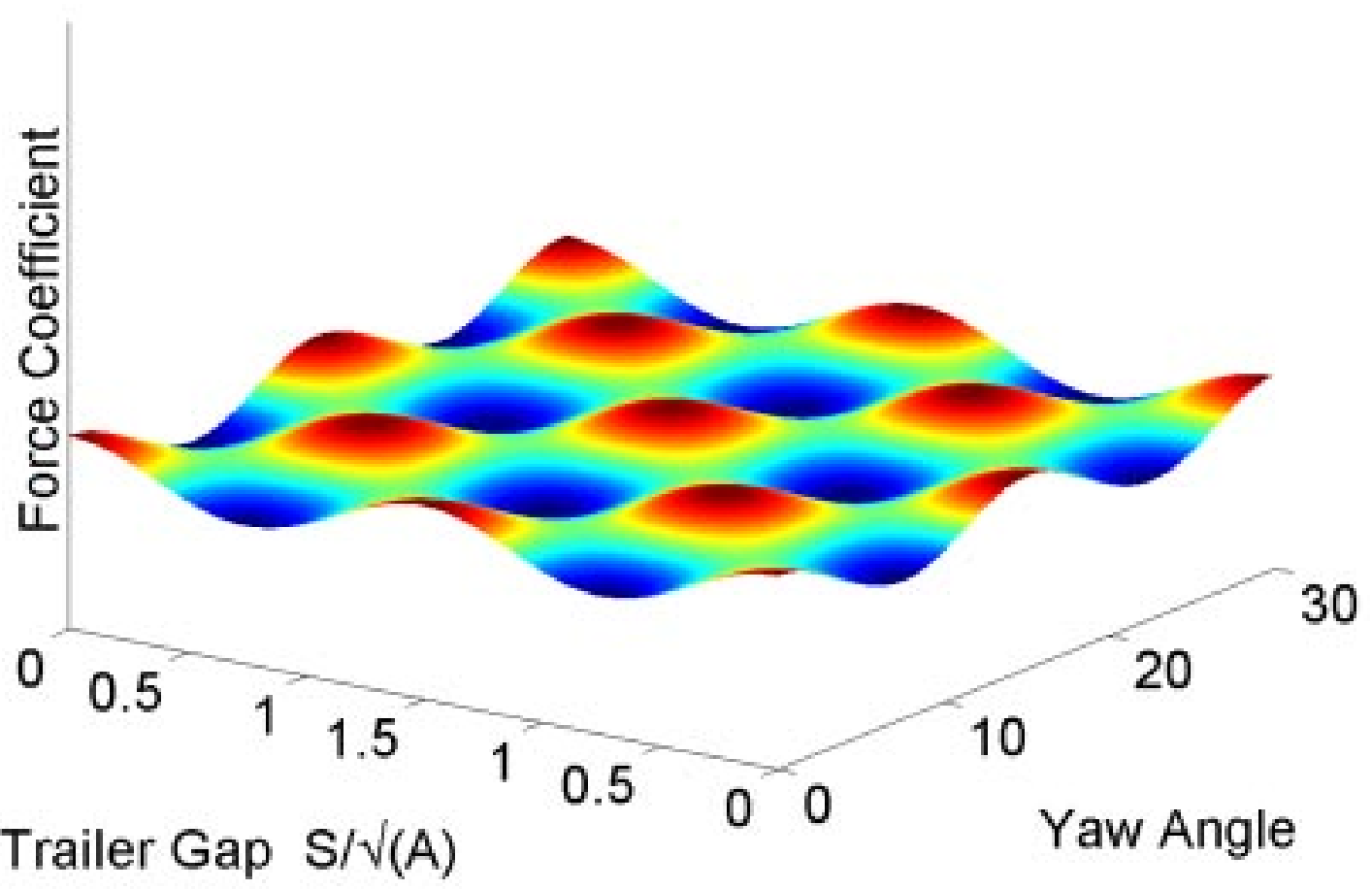




\section{Drag: Cab \& Trailer/w Square Verticals}

\section{Cab}
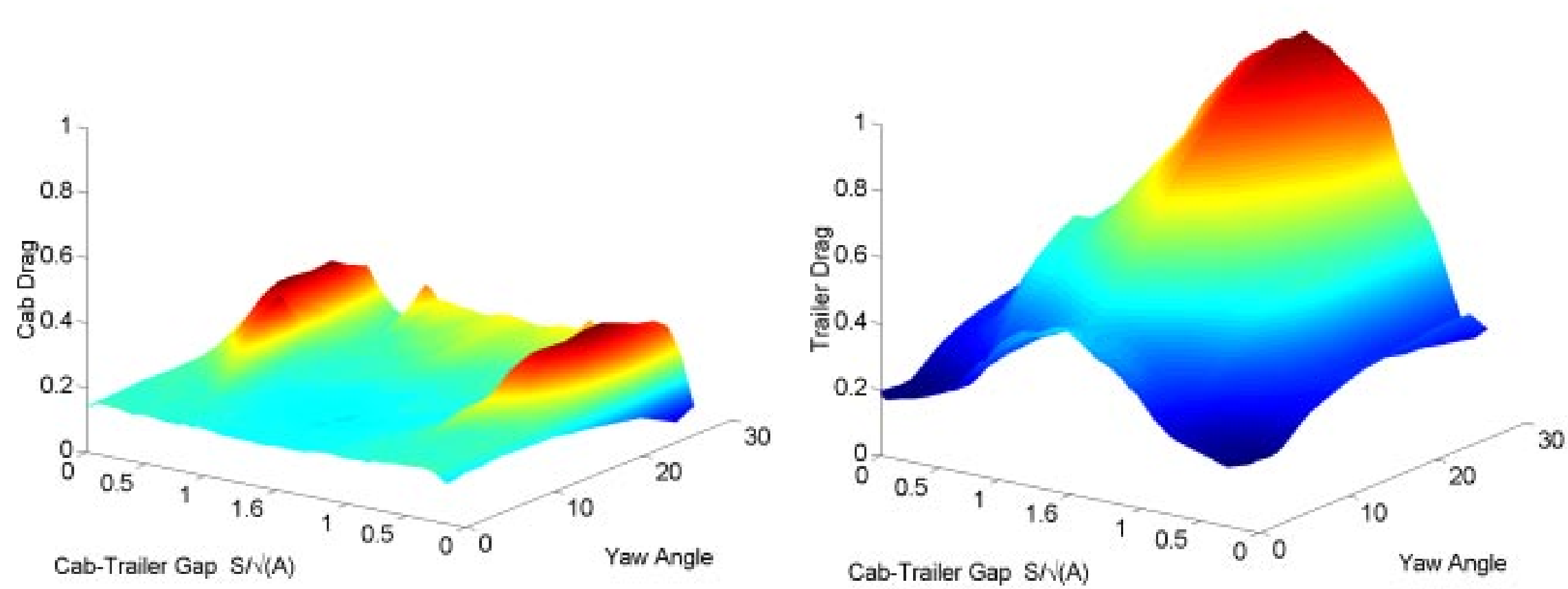

Cab: At small yaw angles, little variation in $C_{D}$ At larger yaw, there is a "critical" gap-- $C_{D}$ more than doubles

Trailer: At small yaw angles, $C_{D}$ values more than double as gap varies Weak influence of "critical" gap 


\section{Drag: Cab \& Trailer/w Rounded Verticals \\ $\mathrm{Cab}$ \\ Trailer}
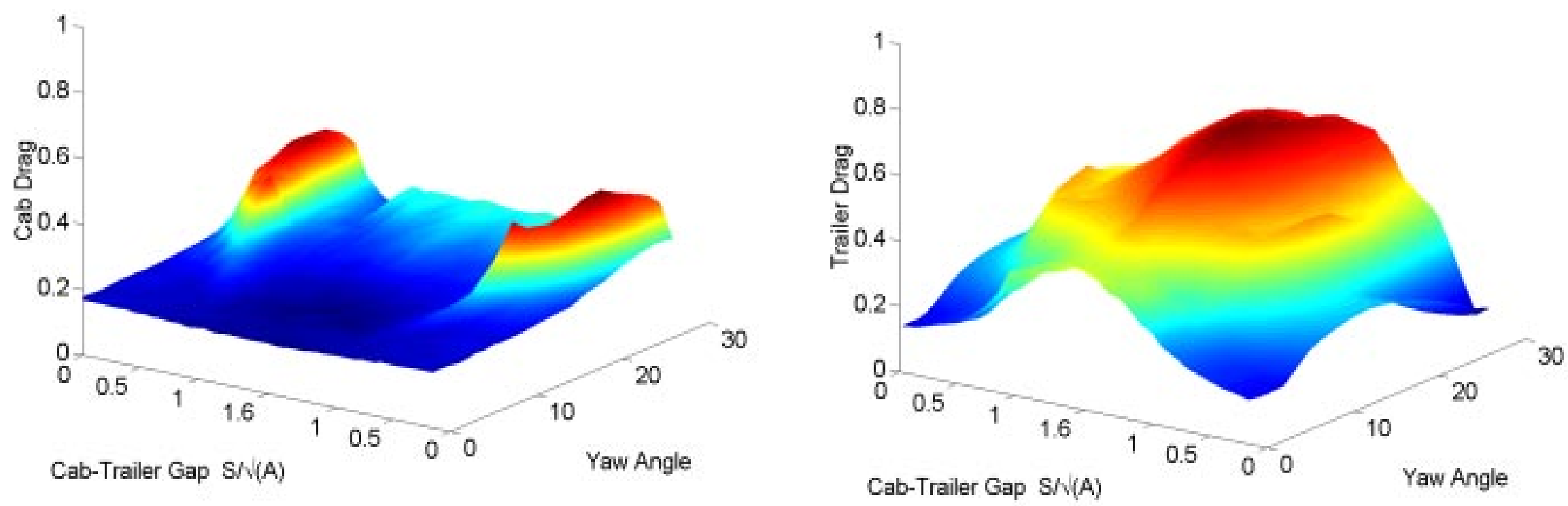

Cab: $\quad$ Rounded verticals on trailer produce higher $\mathrm{C}_{\mathrm{D}}$ 's on cab

"Critical" gap is present

Trailer: Rounded verticals produce lower trailer $C_{D}$ 's

Greater influence of "critical" gap (yellow halo region) 


\section{RMS Side Force: Cab \& Trailer/w Rounded Verticals}

$\mathrm{Cab}$

Trailer
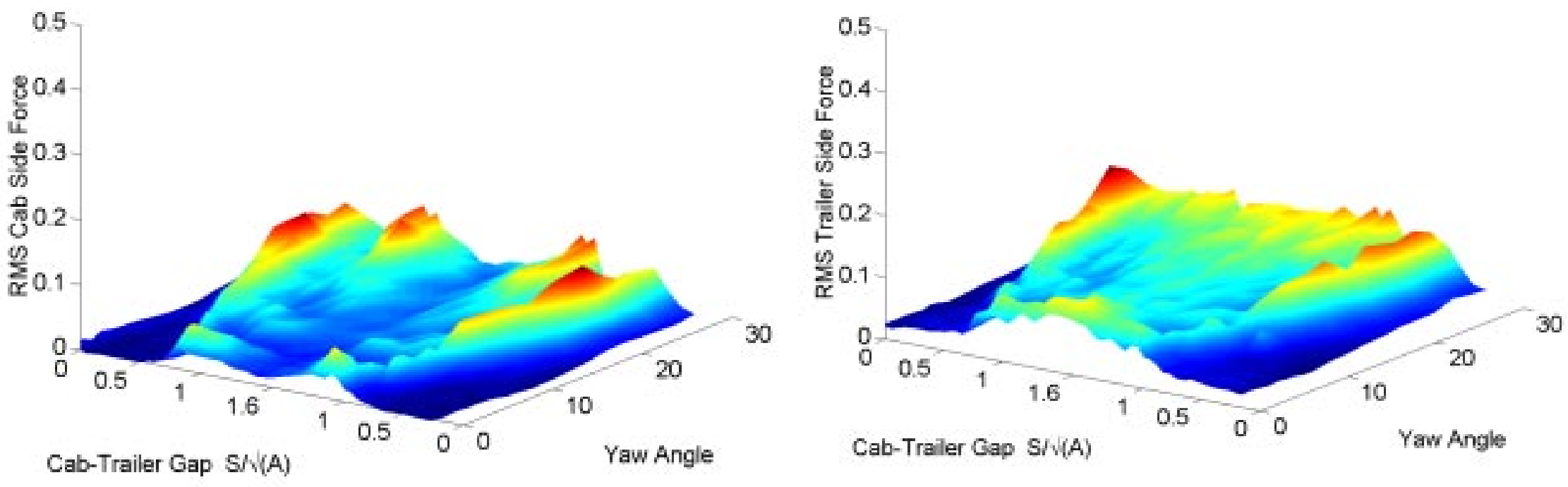

Additional evidence for "critical" gap--side force fluctuations are elevated

Evidence for a second "critical" gap at large yaw angles

Elevated fluctuations at small yaw may be unrelated to fluctuations at larger yaw angles 


\section{RMS Side Force: Cab/w Trip, Wheels \&}

\section{Trailer/w Rounded Verticals, Wheels}

Cab

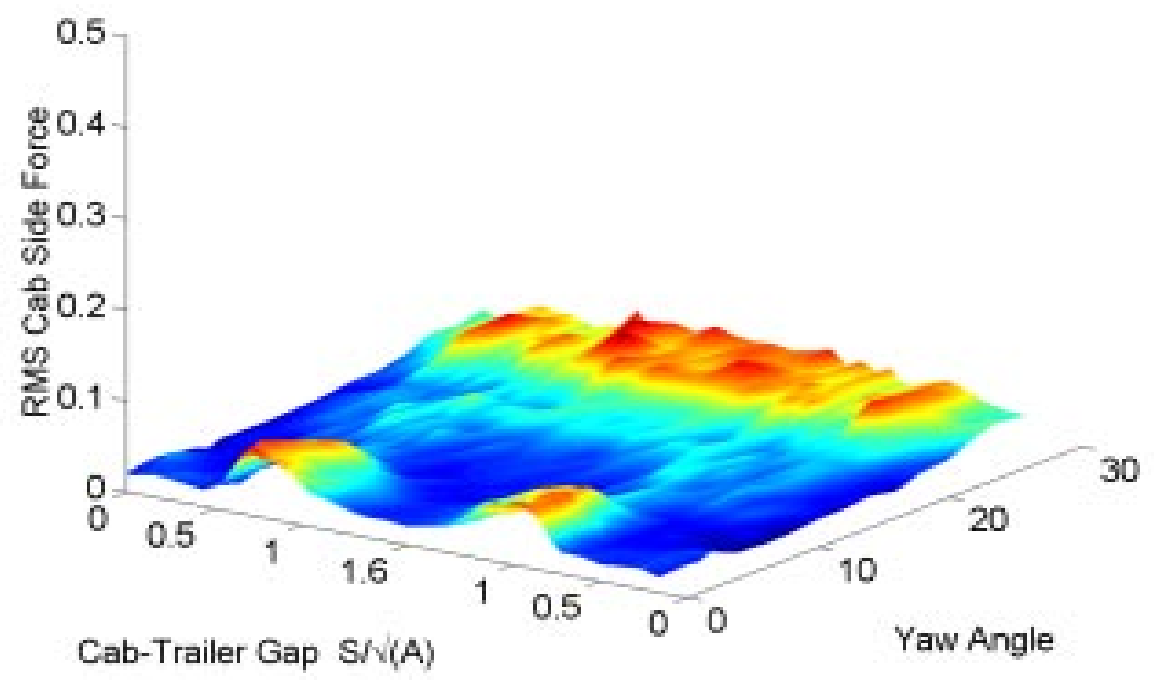

Wheels affect fluctuations at "critical"gap

Note low fluctuation amplitude region separating small yaw and large yaw
Trailer

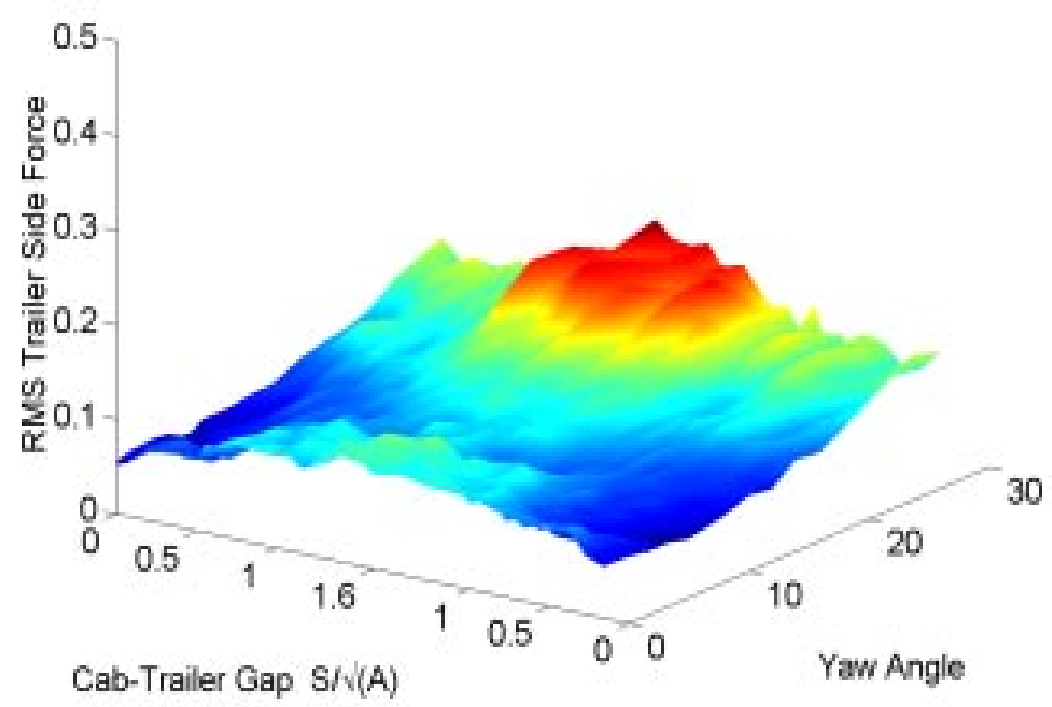




\section{PIV data in horizontal mid-plane}

$$
\mathrm{U}=26 \mathrm{~m} / \mathrm{s}
$$

Tractor

Trailer 


\section{Characterizing gap flow}

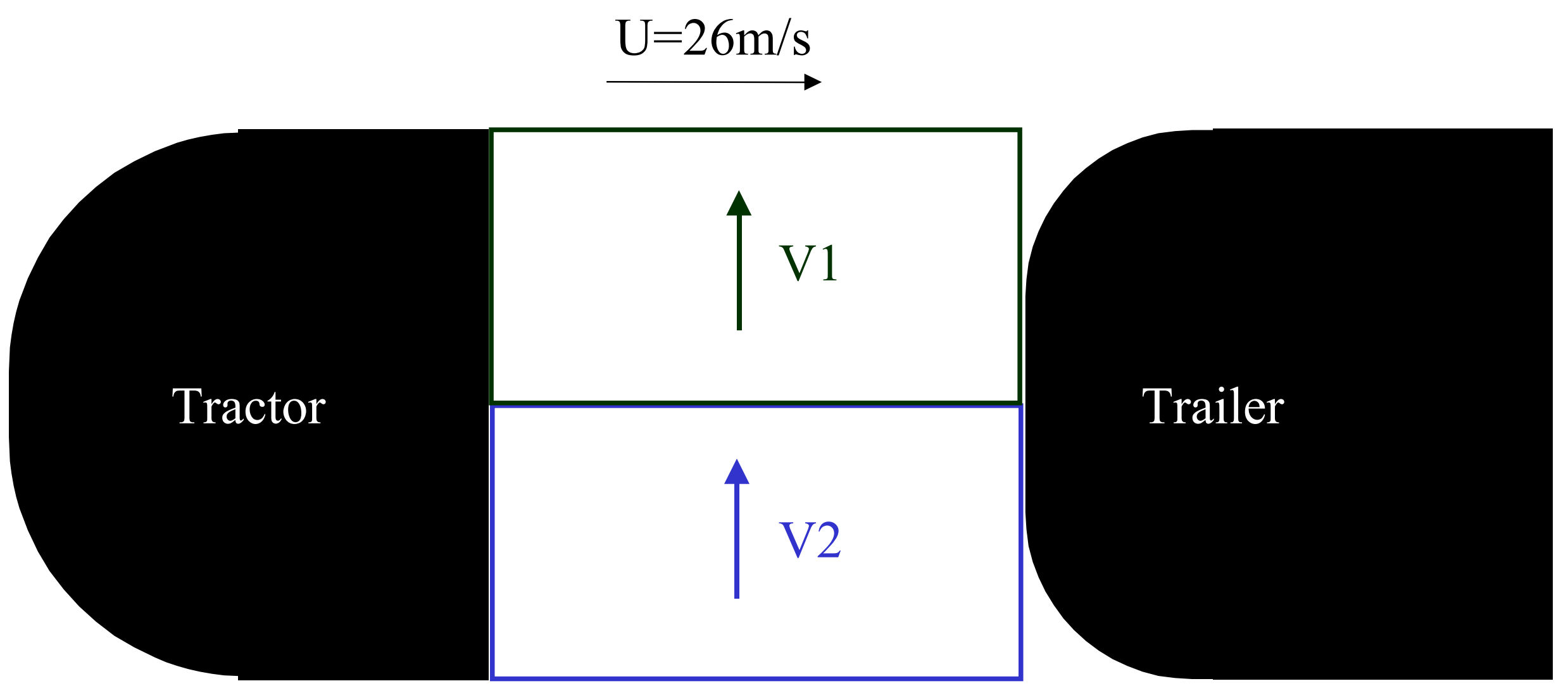




\section{$\alpha=0, \mathrm{G} / \mathrm{L}=0.72$ - State space}

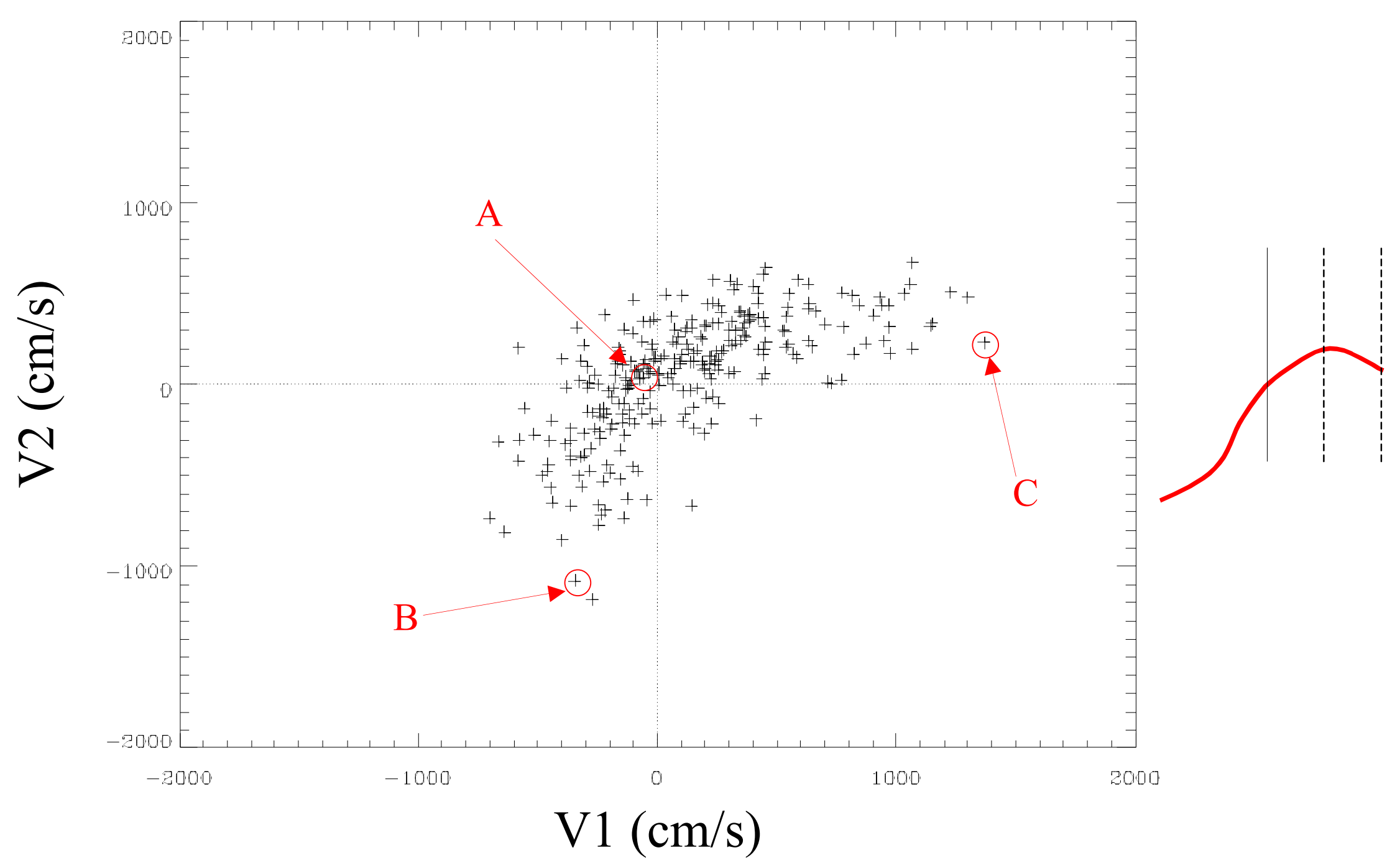




\section{$\alpha=0, \mathrm{G} / \mathrm{L}=0.72$ (Average)}

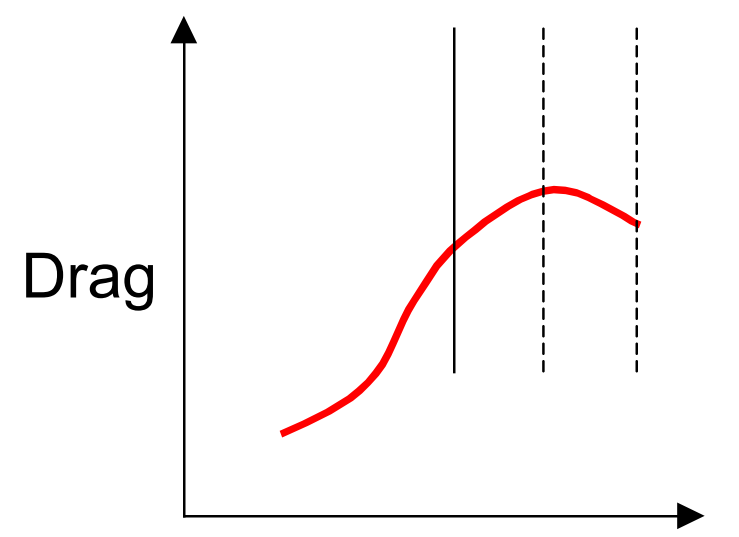

Gap size

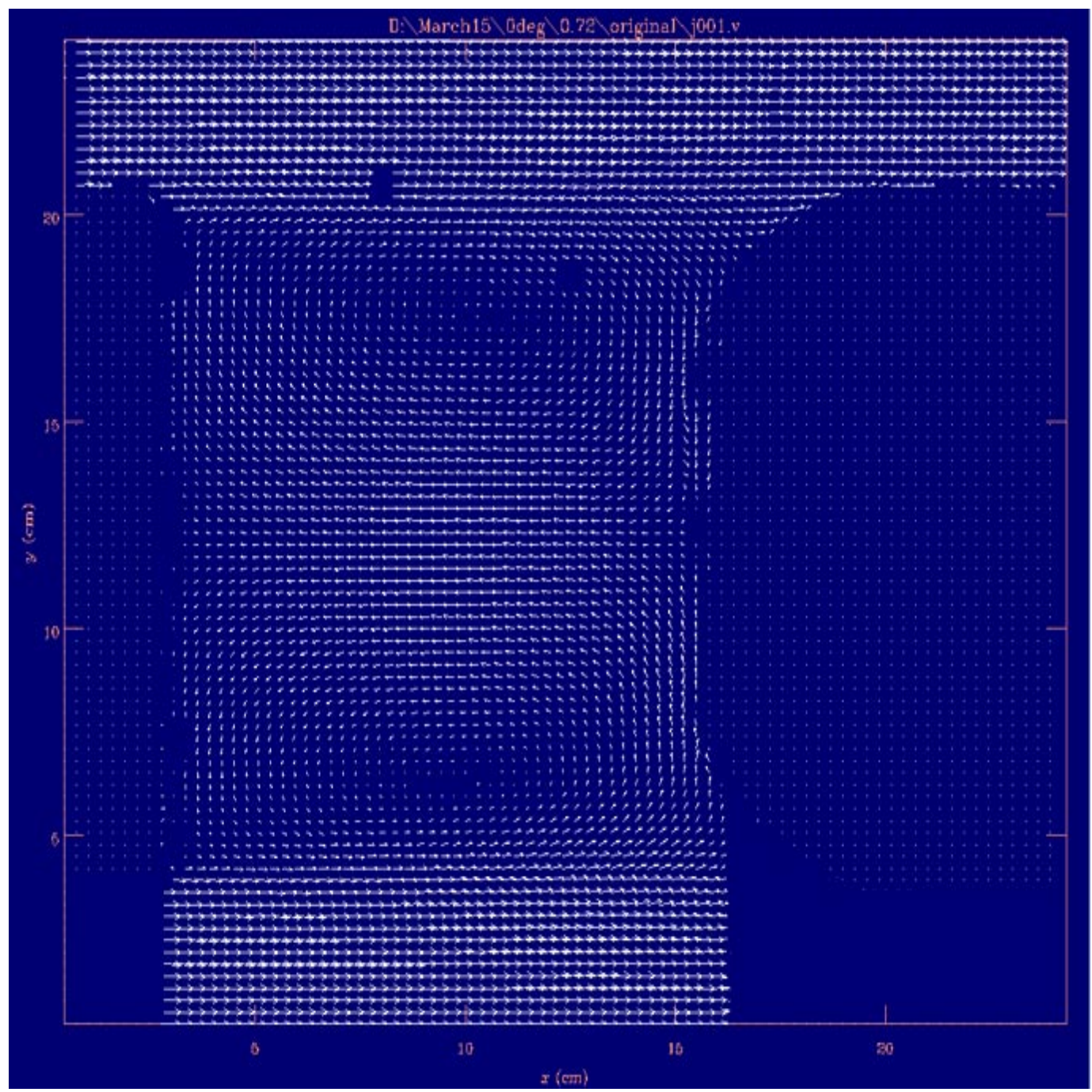




\section{$\alpha=0, \mathrm{G} / \mathrm{L}=0.72$ (sample $\mathrm{A}$ )}
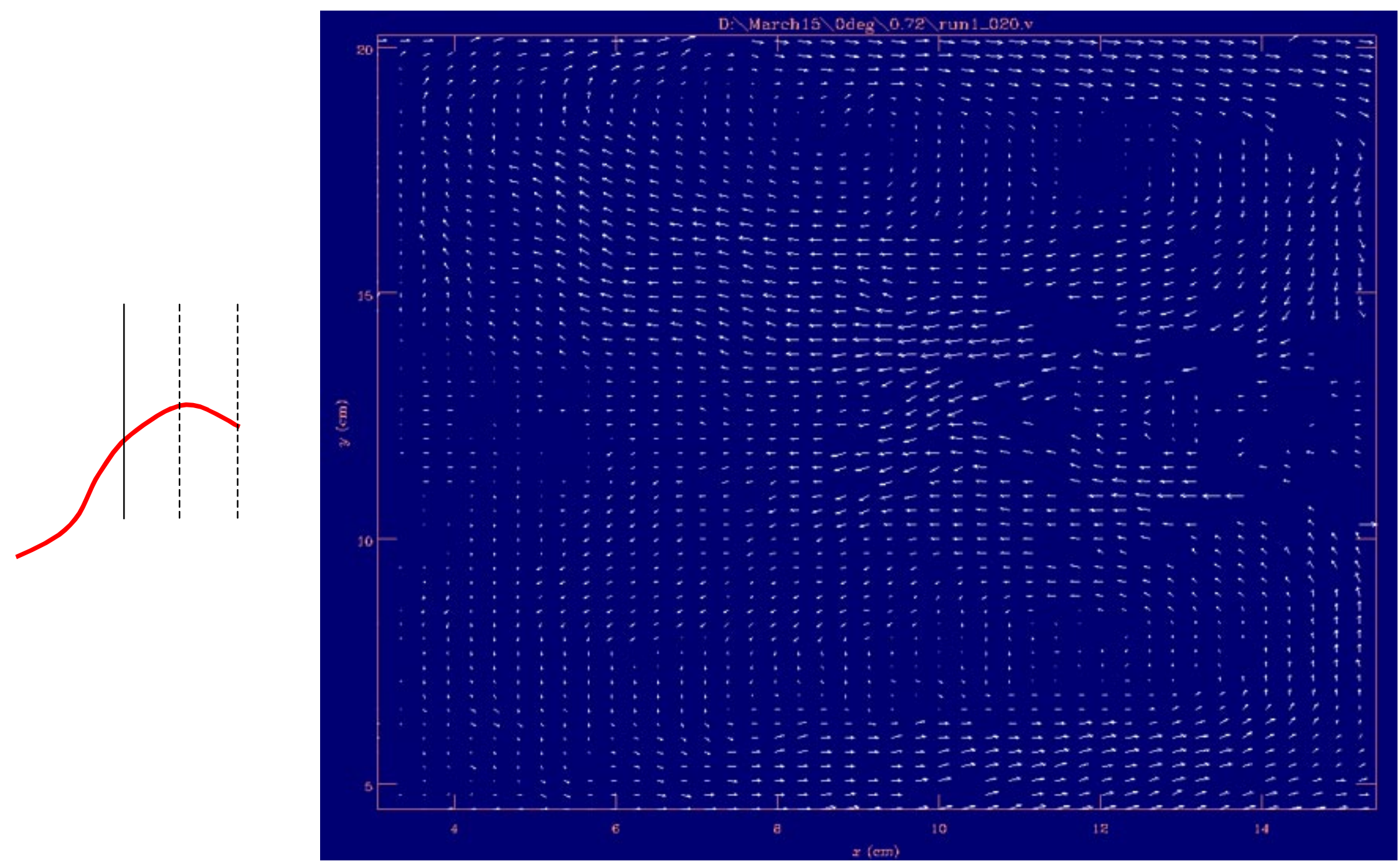


\section{$\alpha=0, \mathrm{G} / \mathrm{L}=0.72$ (sample $\mathrm{B}$ )}
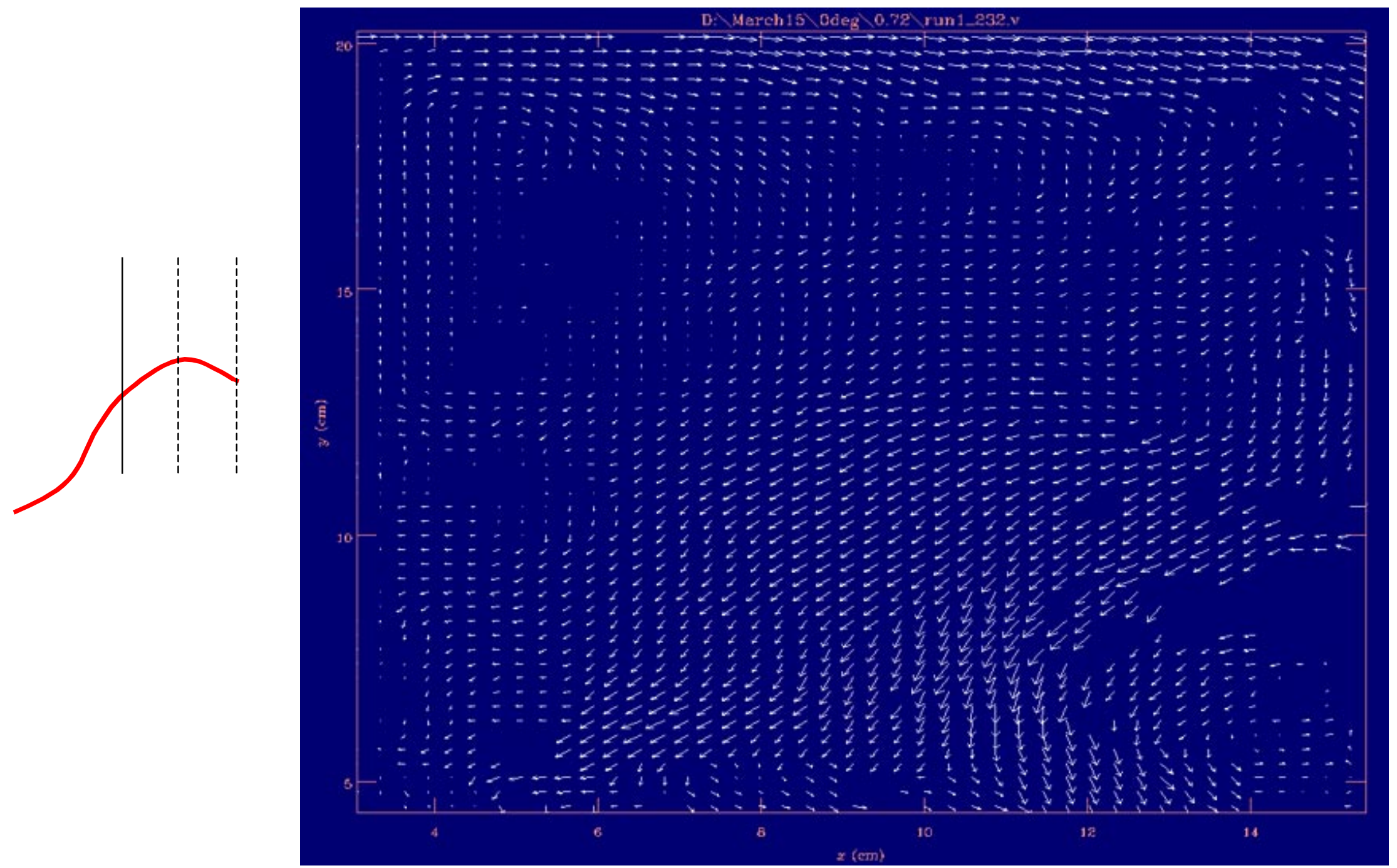


\section{$\alpha=0, \mathrm{G} / \mathrm{L}=0.72$ (sample $\mathrm{C})$}
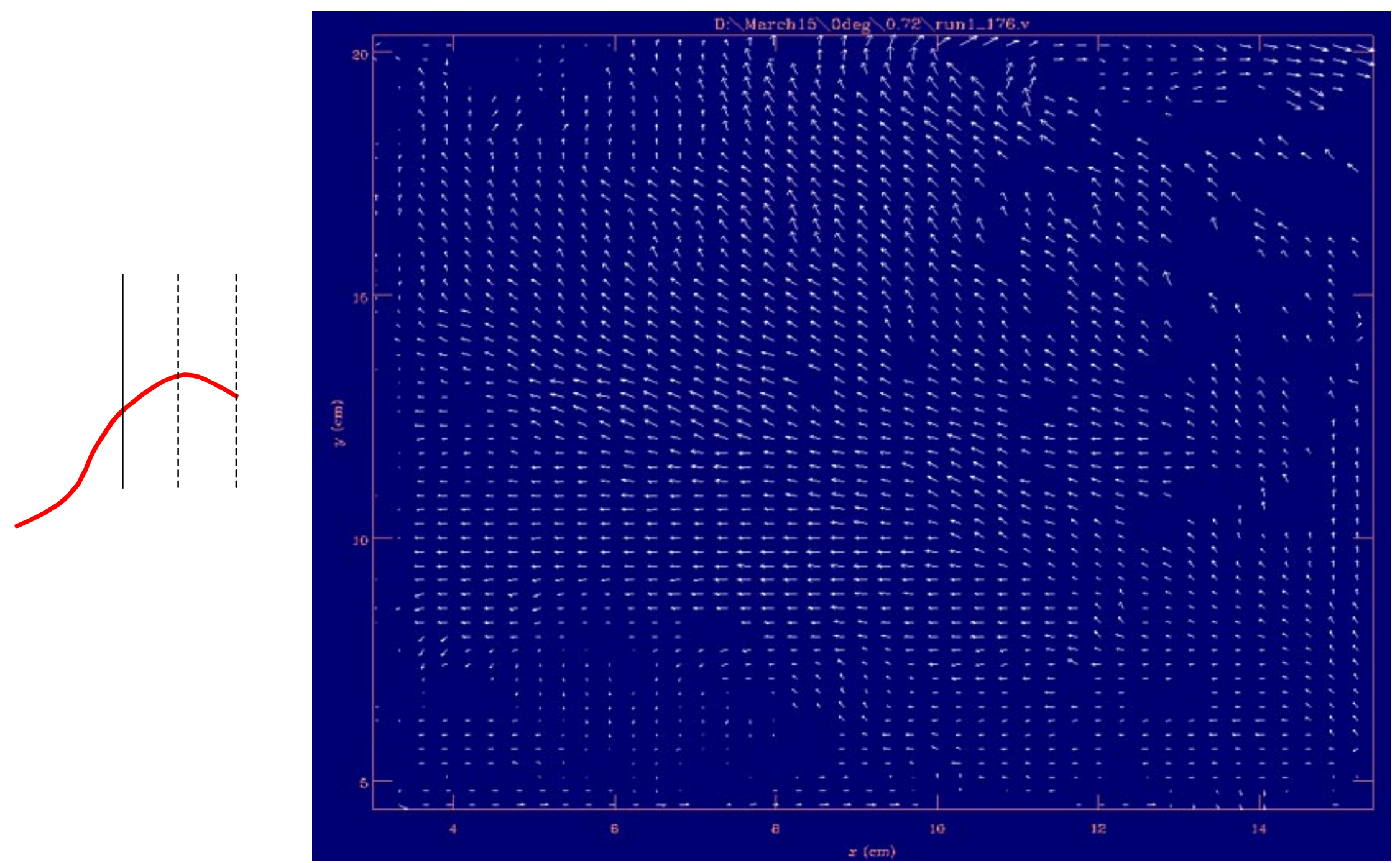


\section{$\alpha=0, \mathrm{G} / \mathrm{L}=0.94$ - State space}

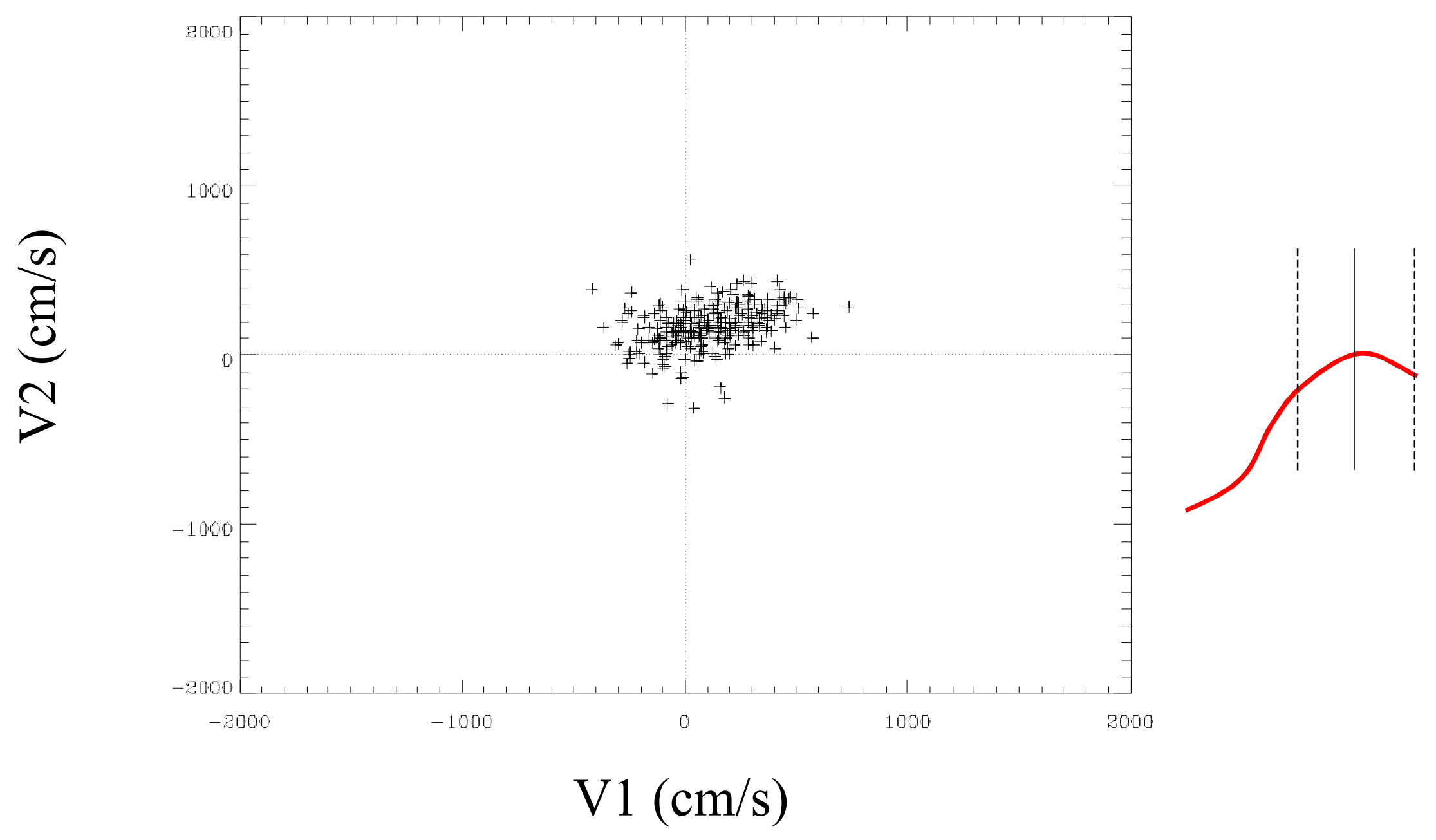




\section{$\alpha=0, \mathrm{G} / \mathrm{L}=1.22$ - State space}

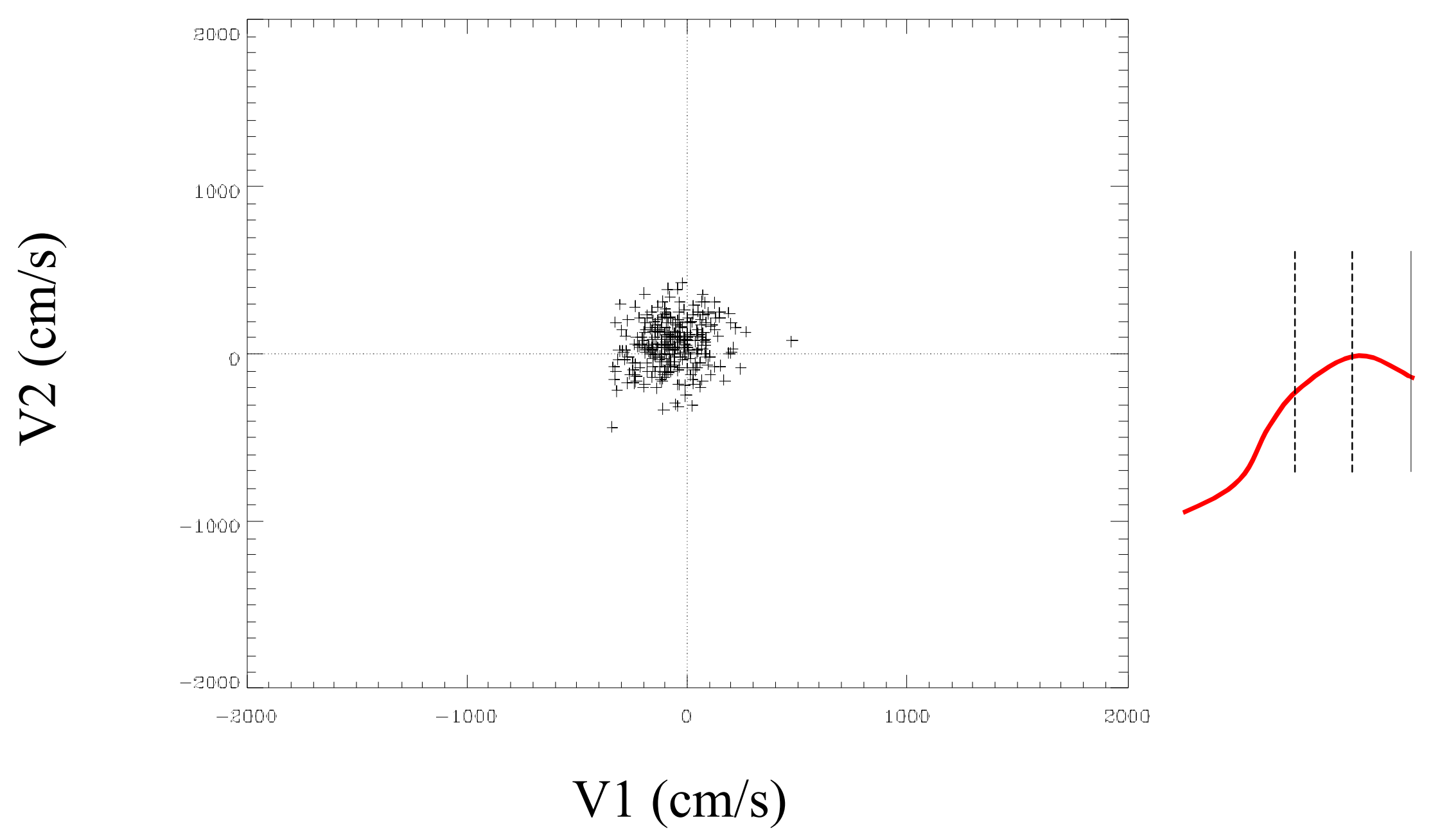


Finite yaw

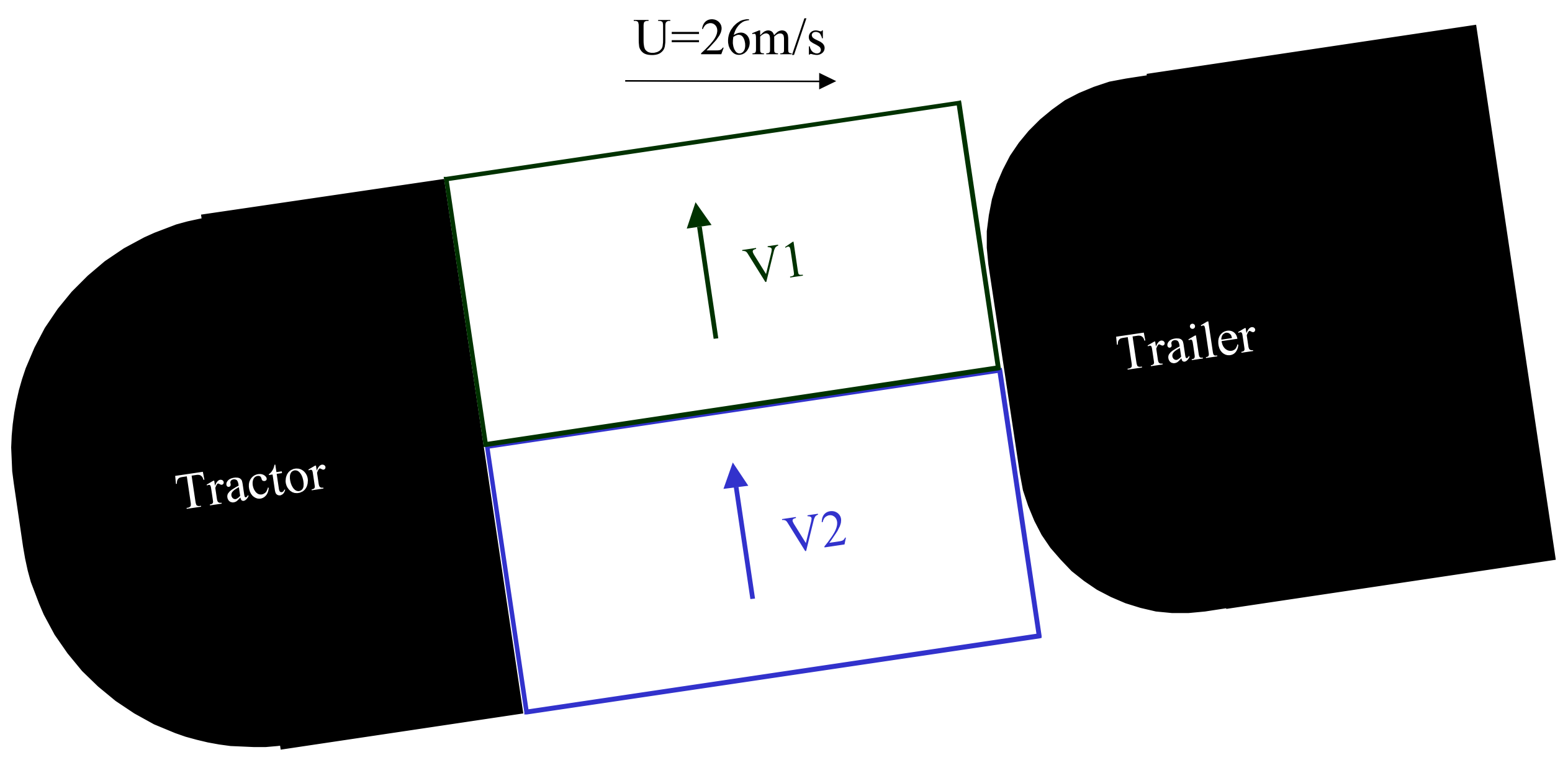




\section{$\alpha=2 \operatorname{deg}, \mathrm{G} / \mathrm{L}=0.60$ (Average)}
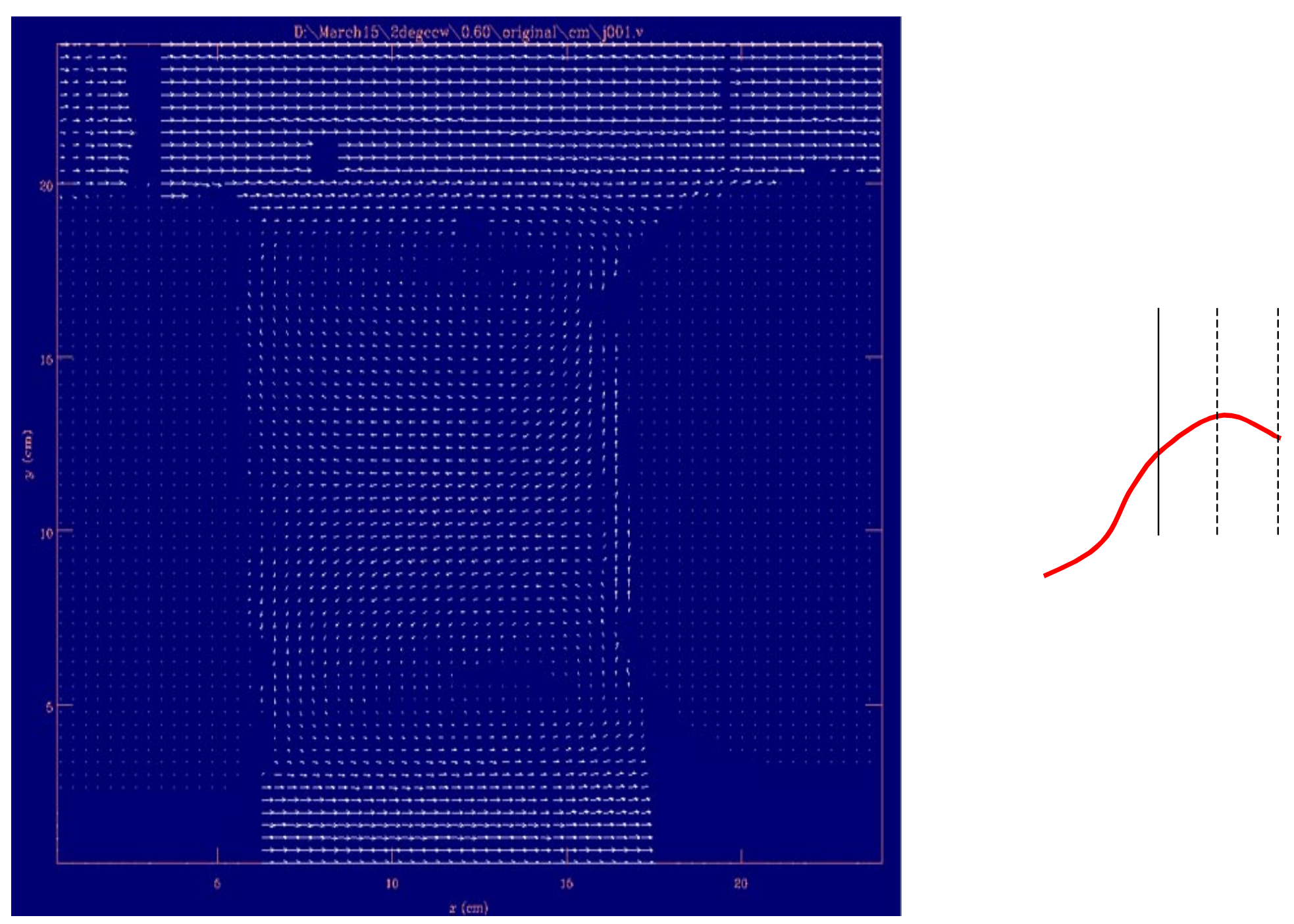


\section{$\alpha=2 \mathrm{deg}, \mathrm{G} / \mathrm{L}=0.60$ - State space}

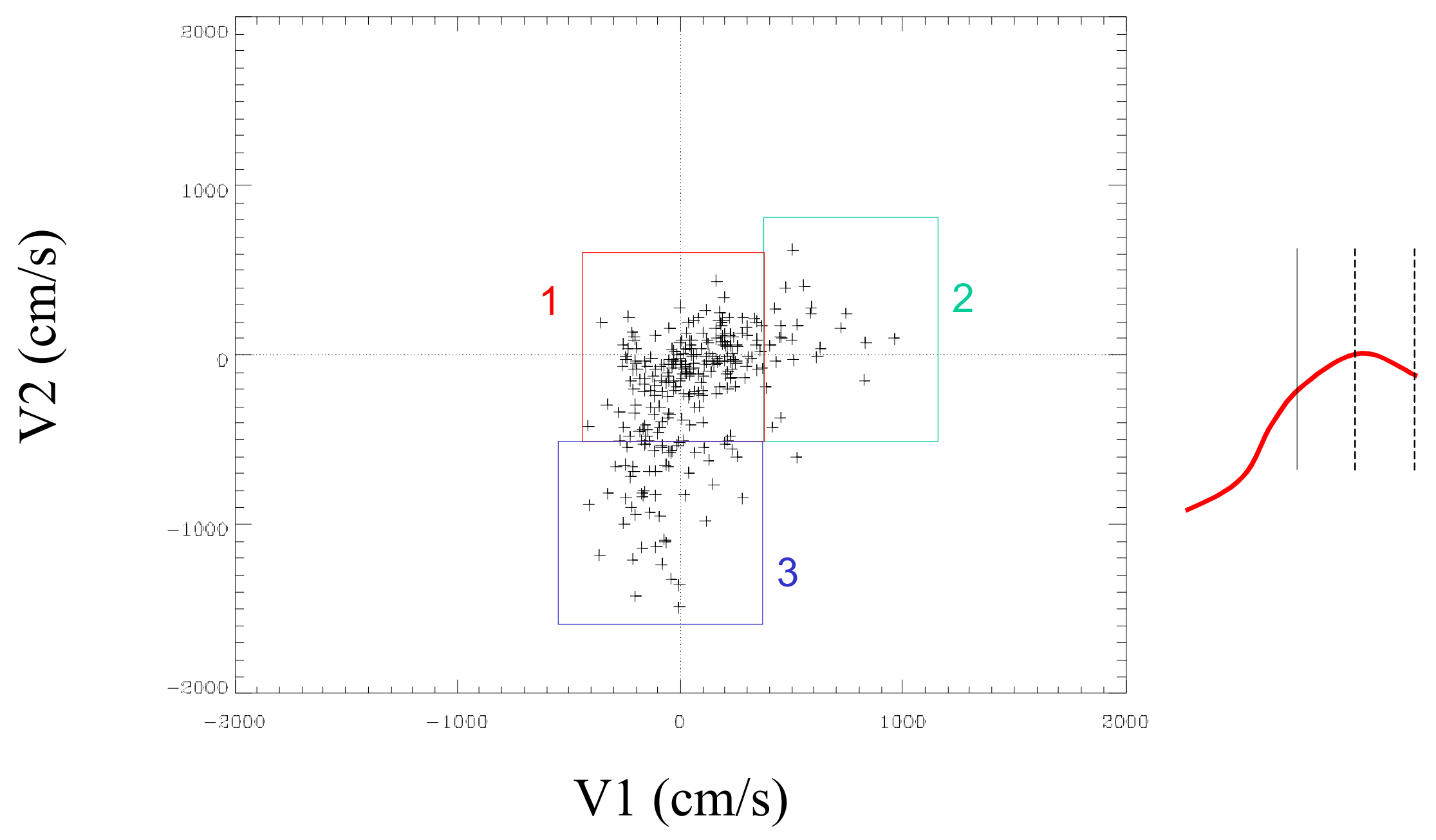




\section{$\alpha=2 \operatorname{deg}, \mathrm{G} / \mathrm{L}=0.60$ (ensemble average 1)}
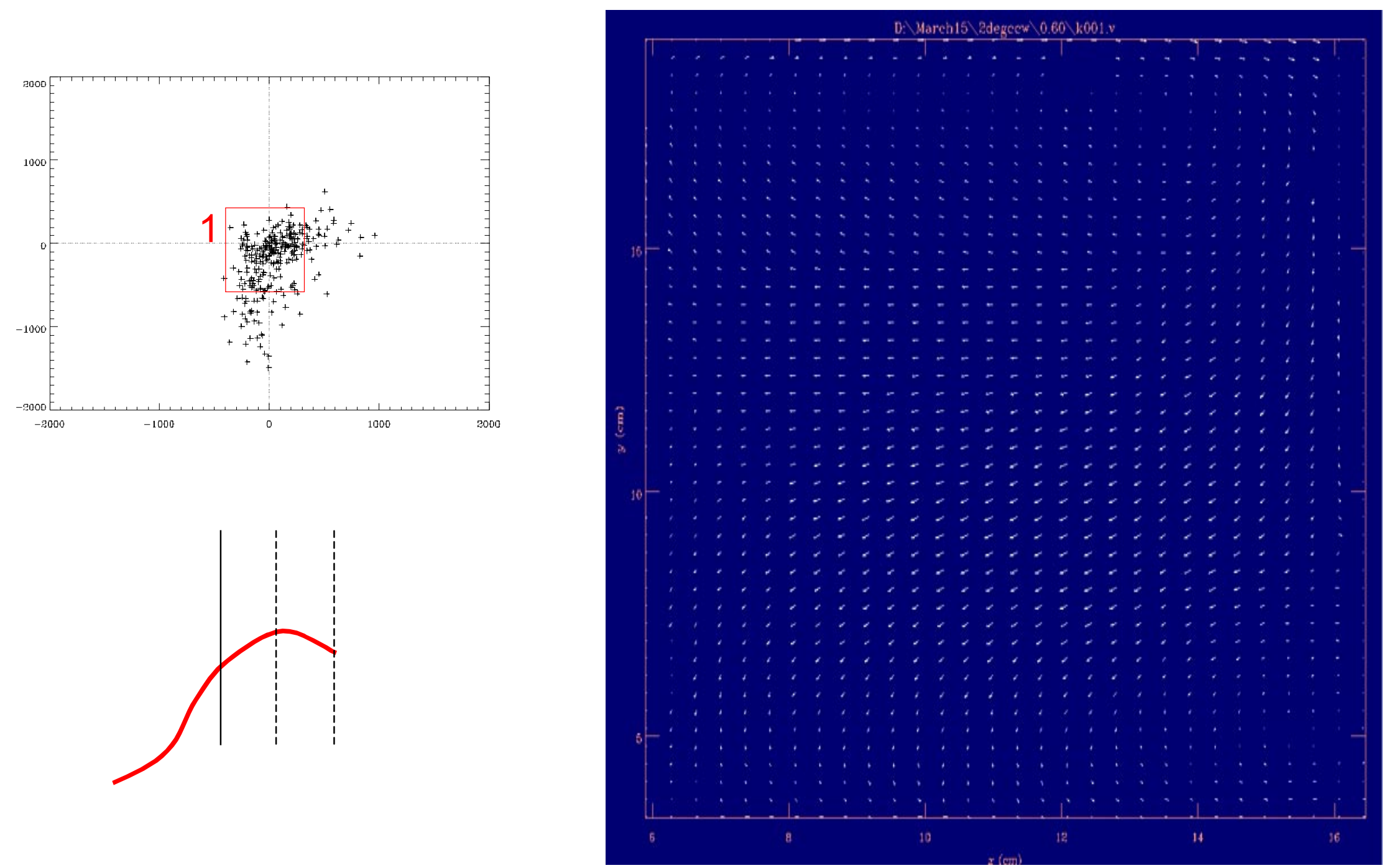


\section{$\alpha=2 \mathrm{deg}, \mathrm{G} / \mathrm{L}=0.60$ (ensemble average 2)}
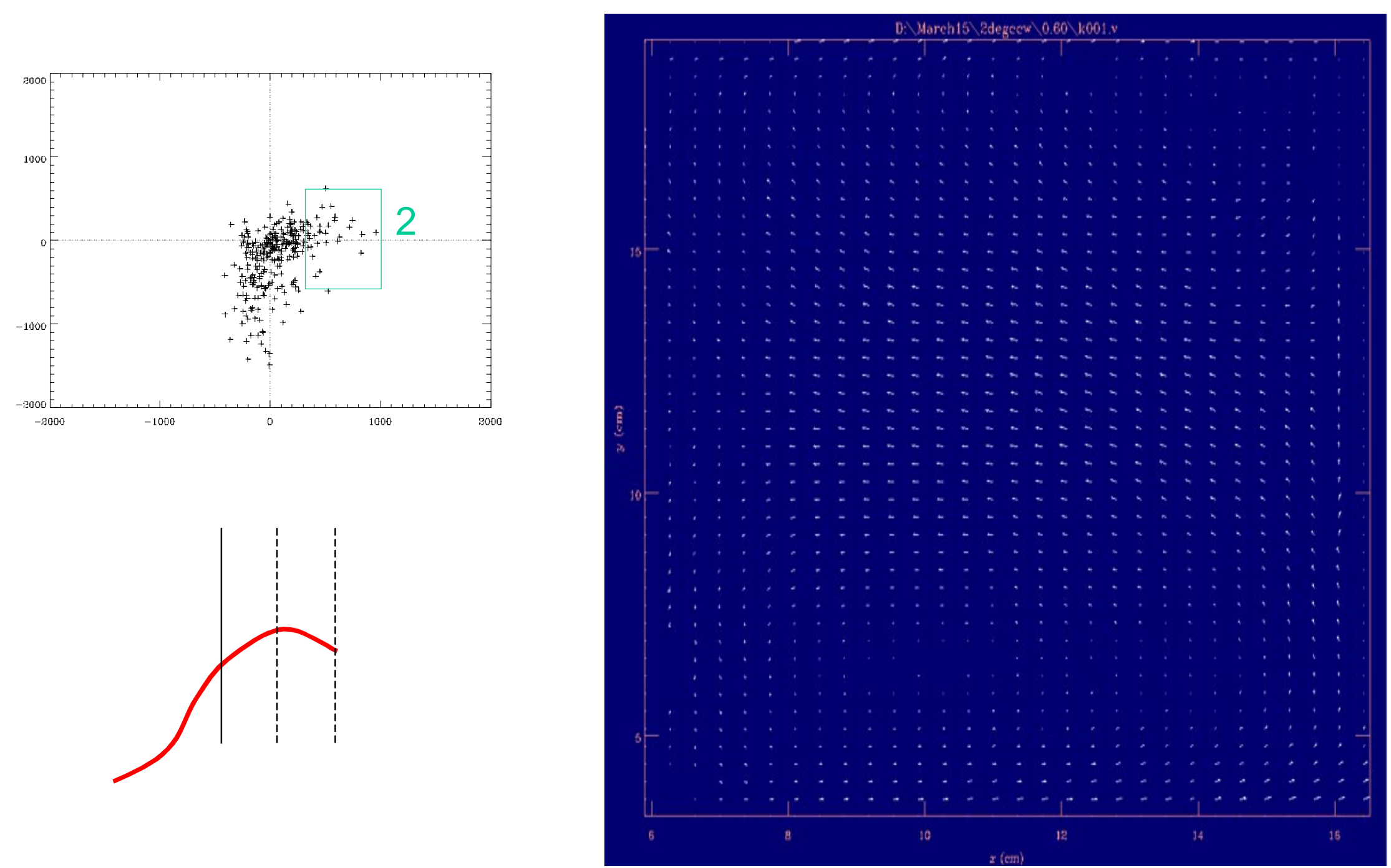


\section{$\alpha=2 \mathrm{deg}, \mathrm{G} / \mathrm{L}=0.60$ (ensemble average 3 )}
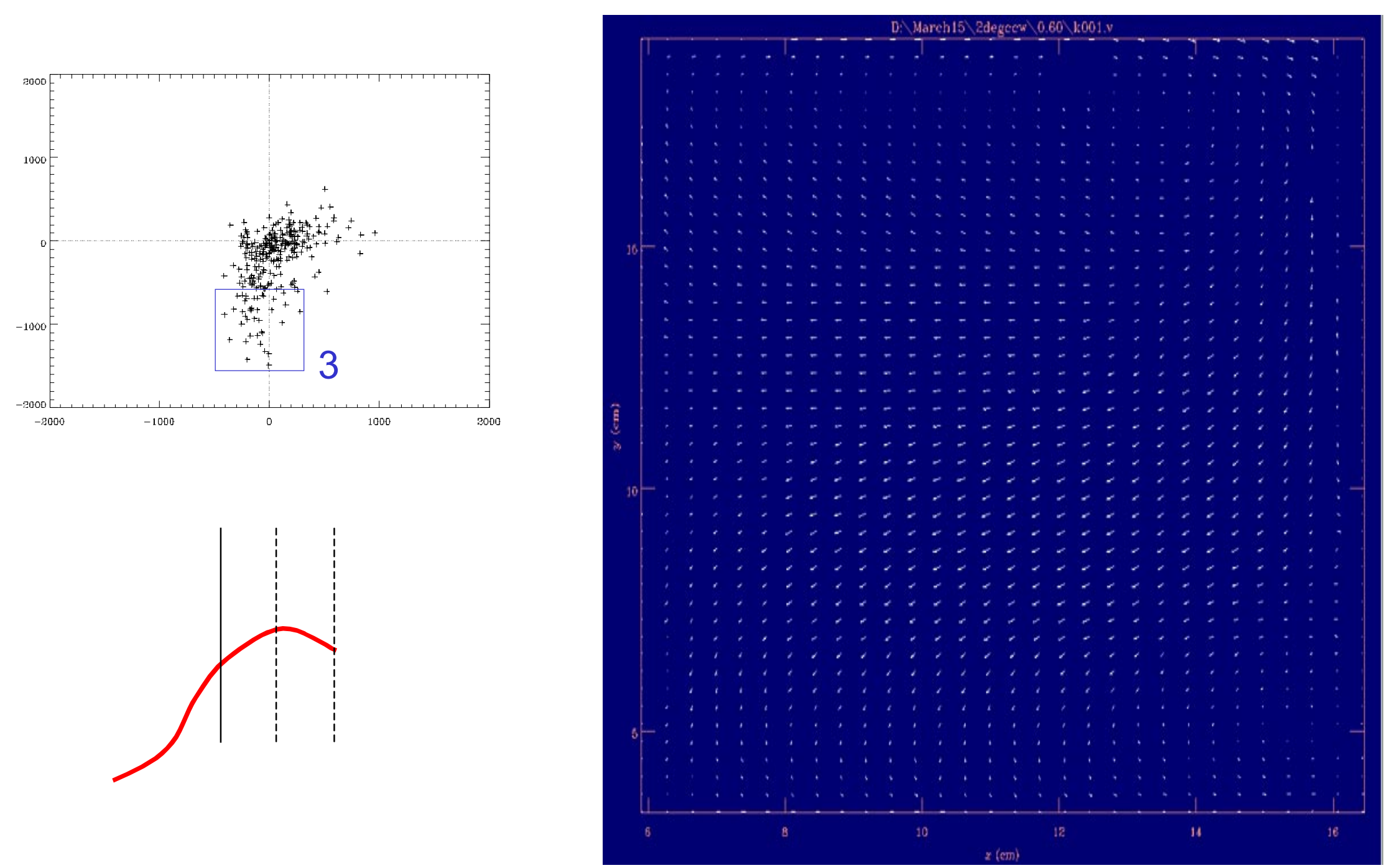


\section{$\alpha=2 \mathrm{deg}, \mathrm{G} / \mathrm{L}=0.80$ - State space}

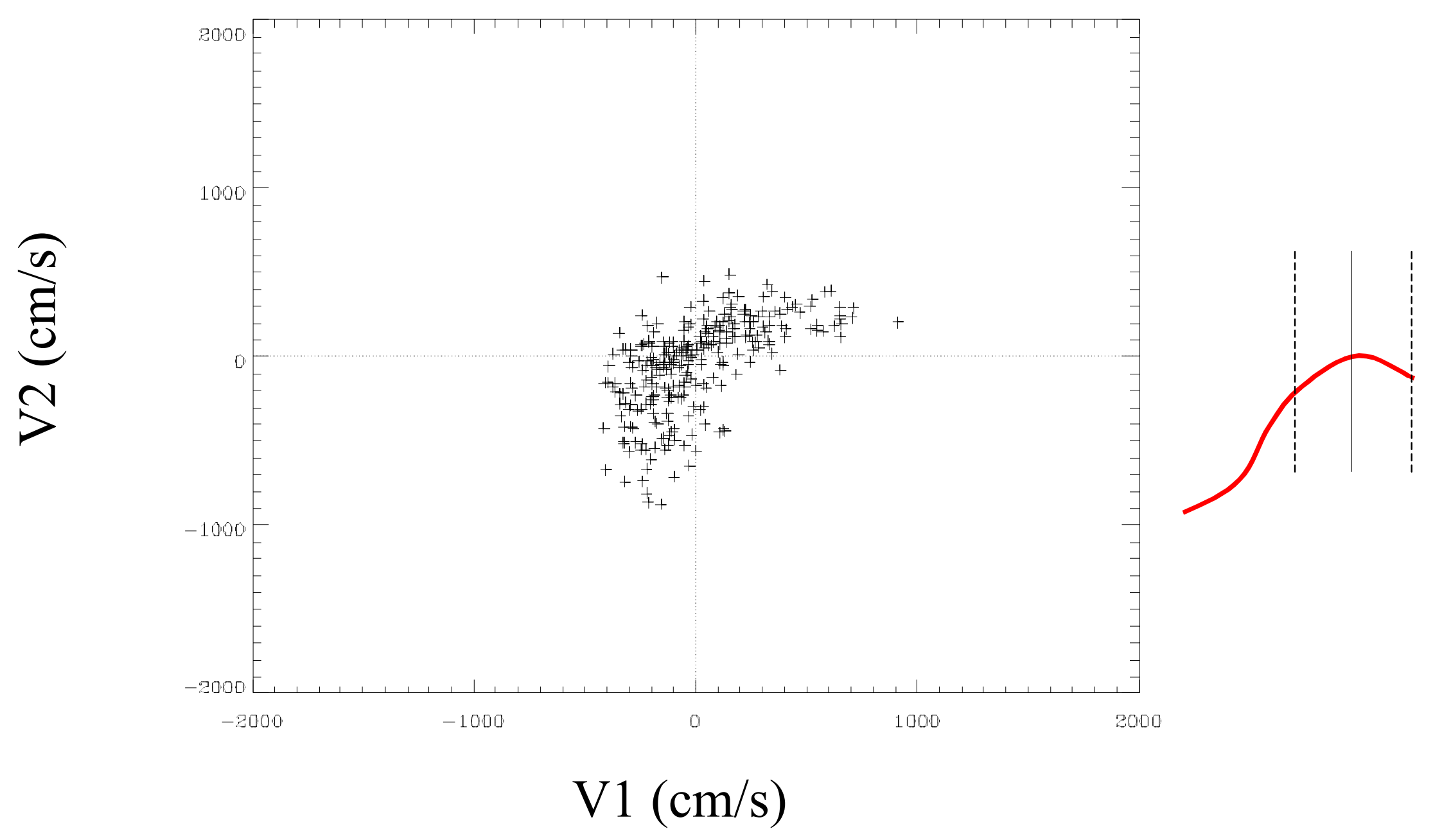




\section{$\alpha=2 \mathrm{deg}, \mathrm{G} / \mathrm{L}=1.00$ - State space}

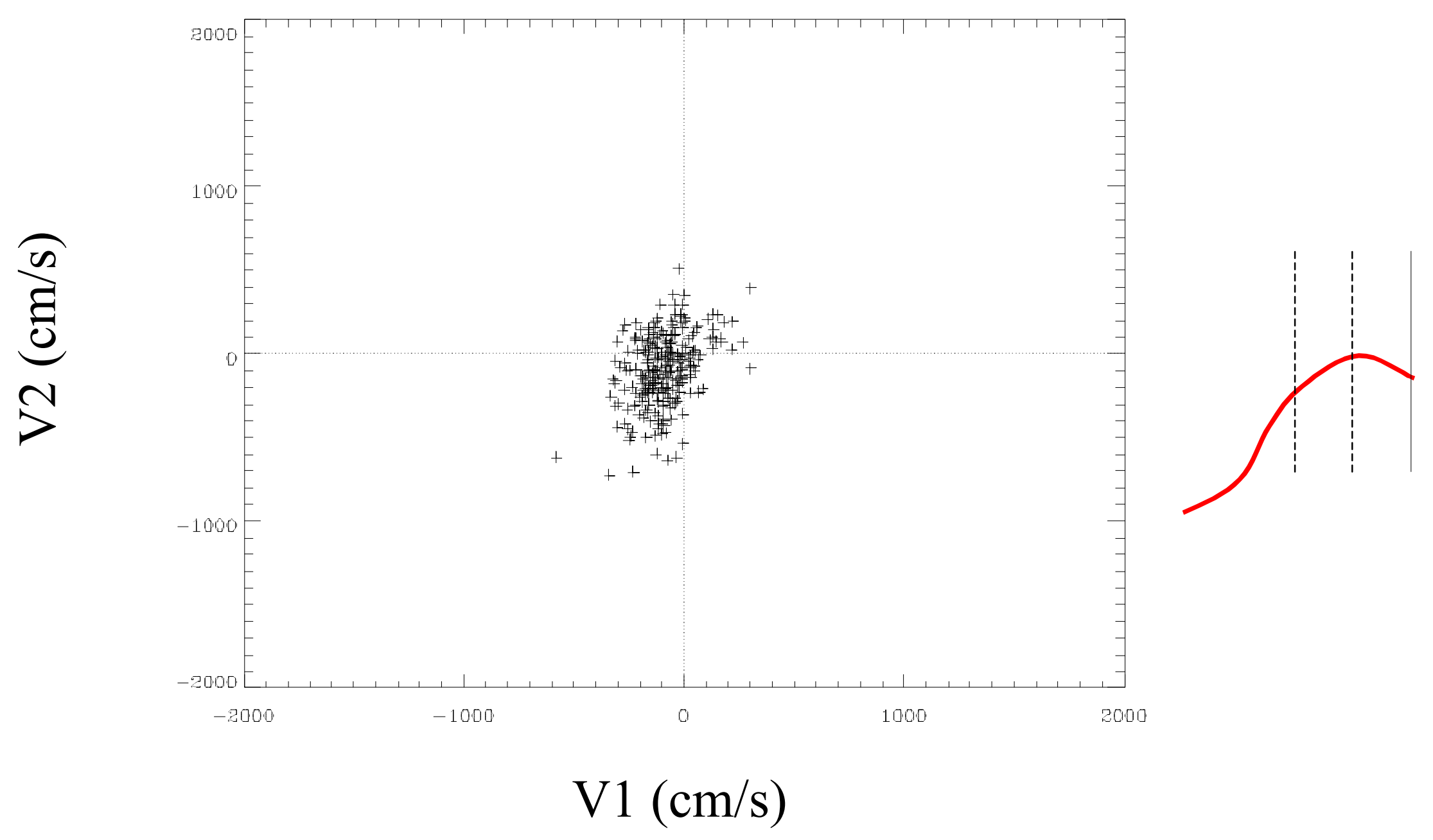




\section{$\alpha=6 \mathrm{deg}, \mathrm{G} / \mathrm{L}=0.50$ - State space}

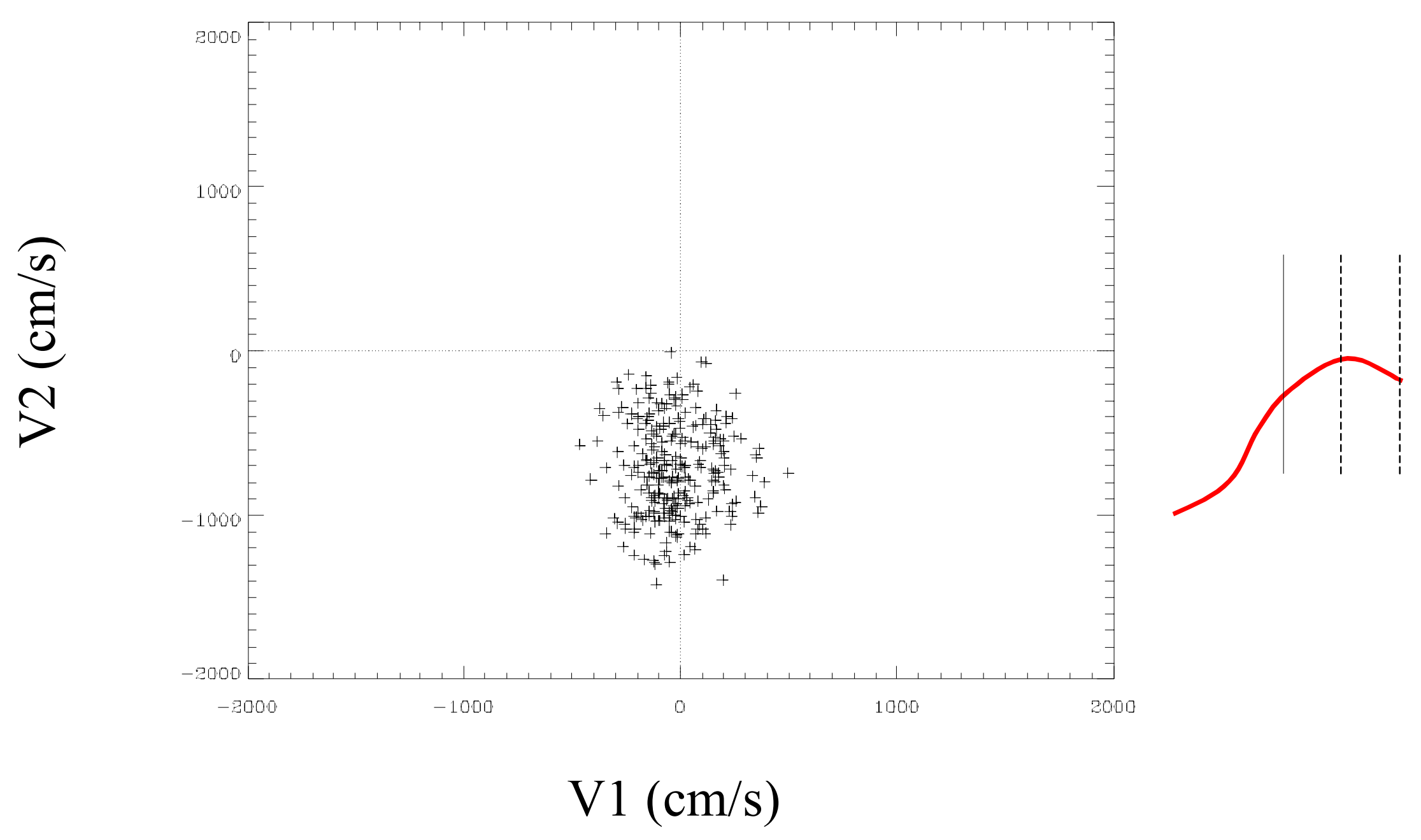




\section{$\alpha=6 \mathrm{deg}, \mathrm{G} / \mathrm{L}=0.65$ - State space}

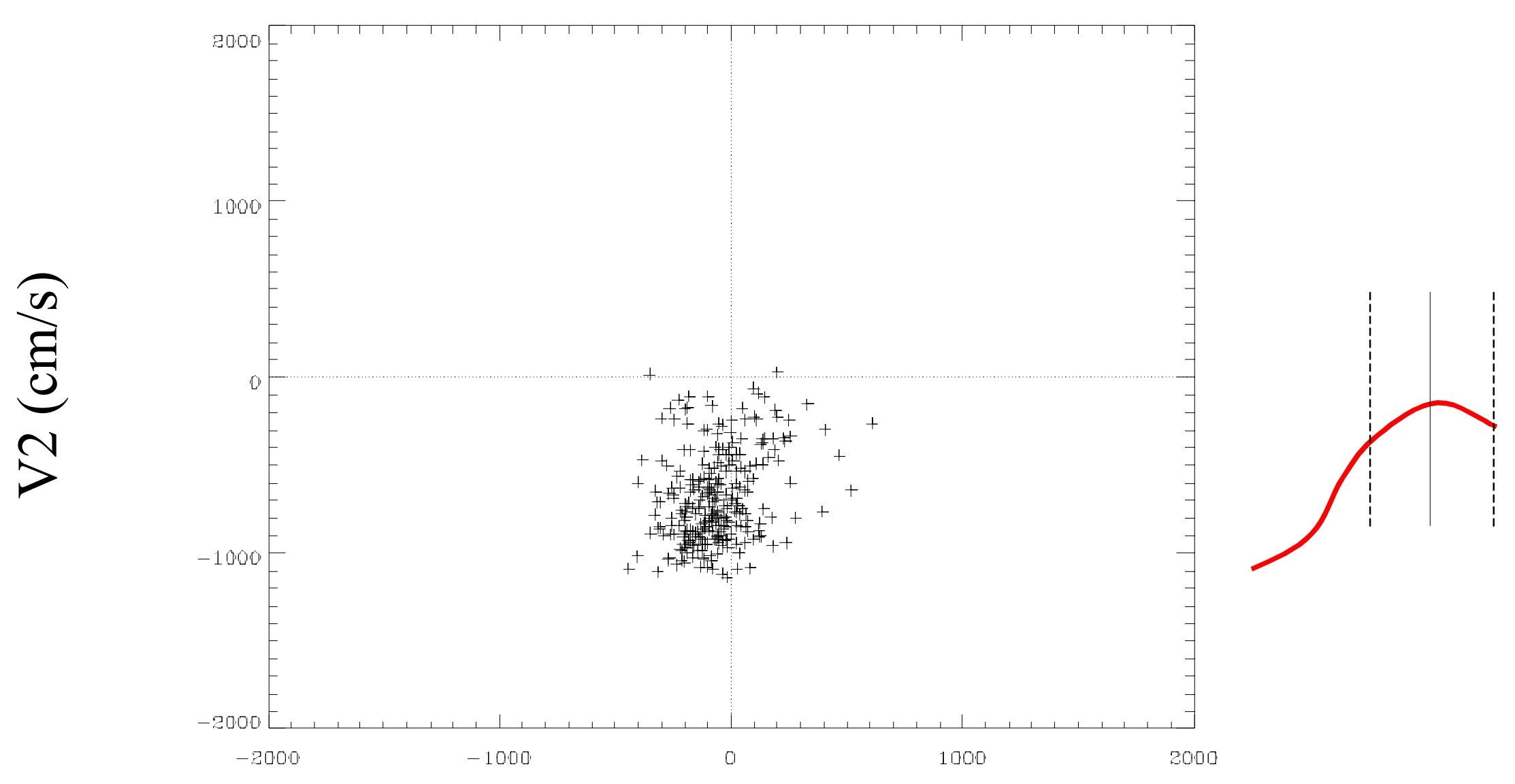

$\mathrm{V} 1(\mathrm{~cm} / \mathrm{s})$ 


\section{$\alpha=6 \mathrm{deg}, \mathrm{G} / \mathrm{L}=0.80$ - State space}

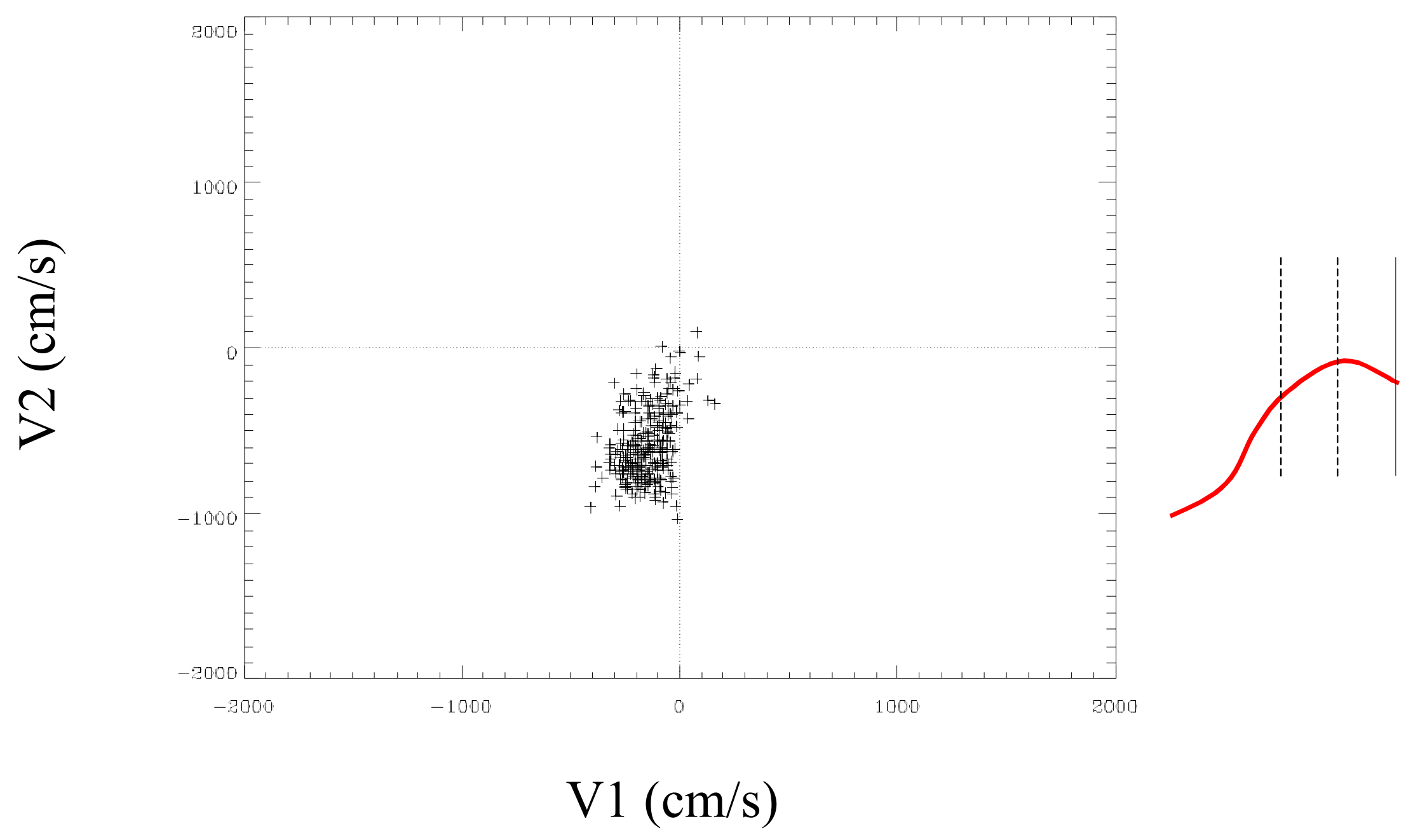




\section{Effect of Gap spacing on drag (1/3)}

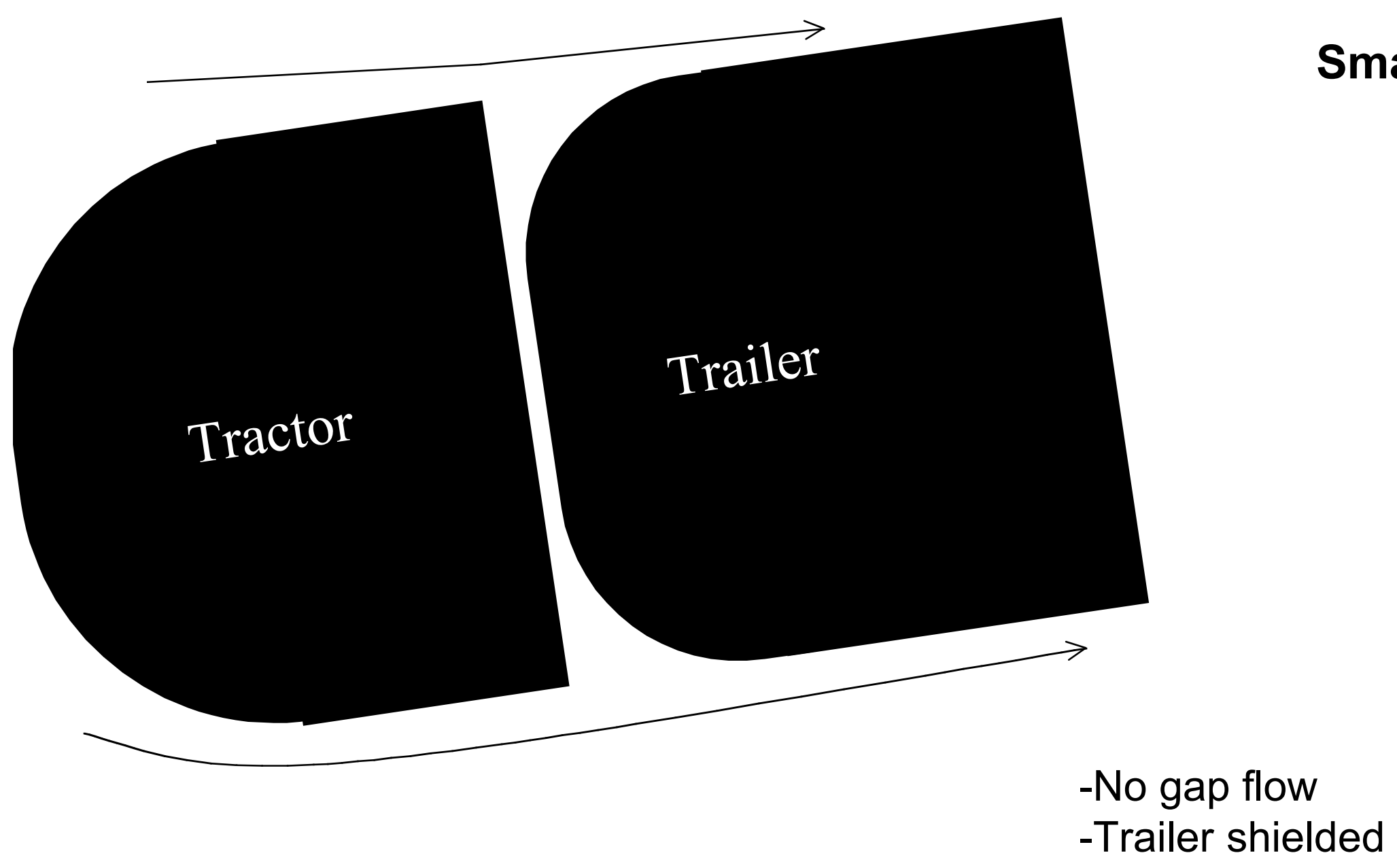




\section{Effect of Gap spacing on drag (2/3)}

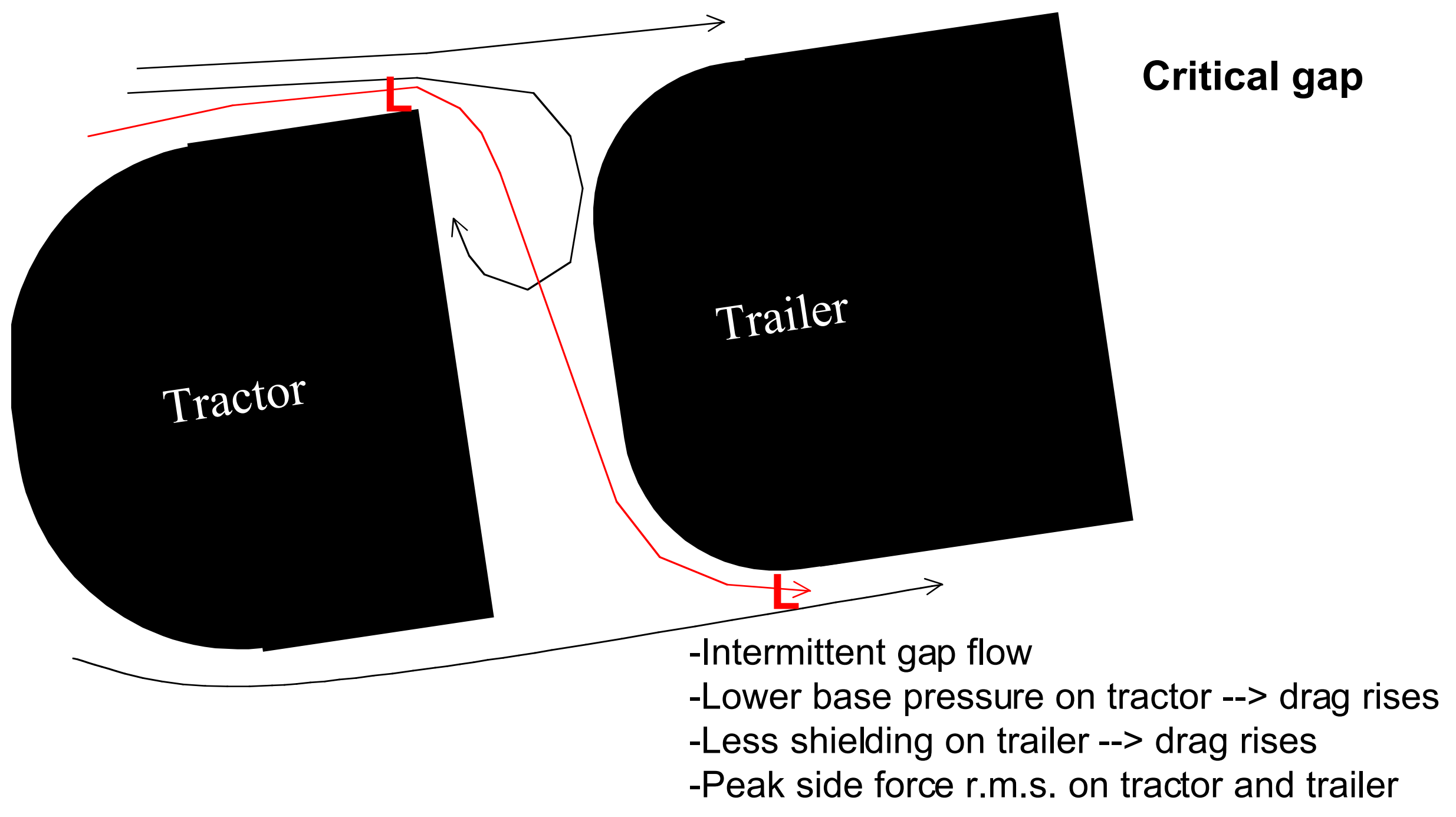




\section{Effect of Gap spacing on drag (3/3)}

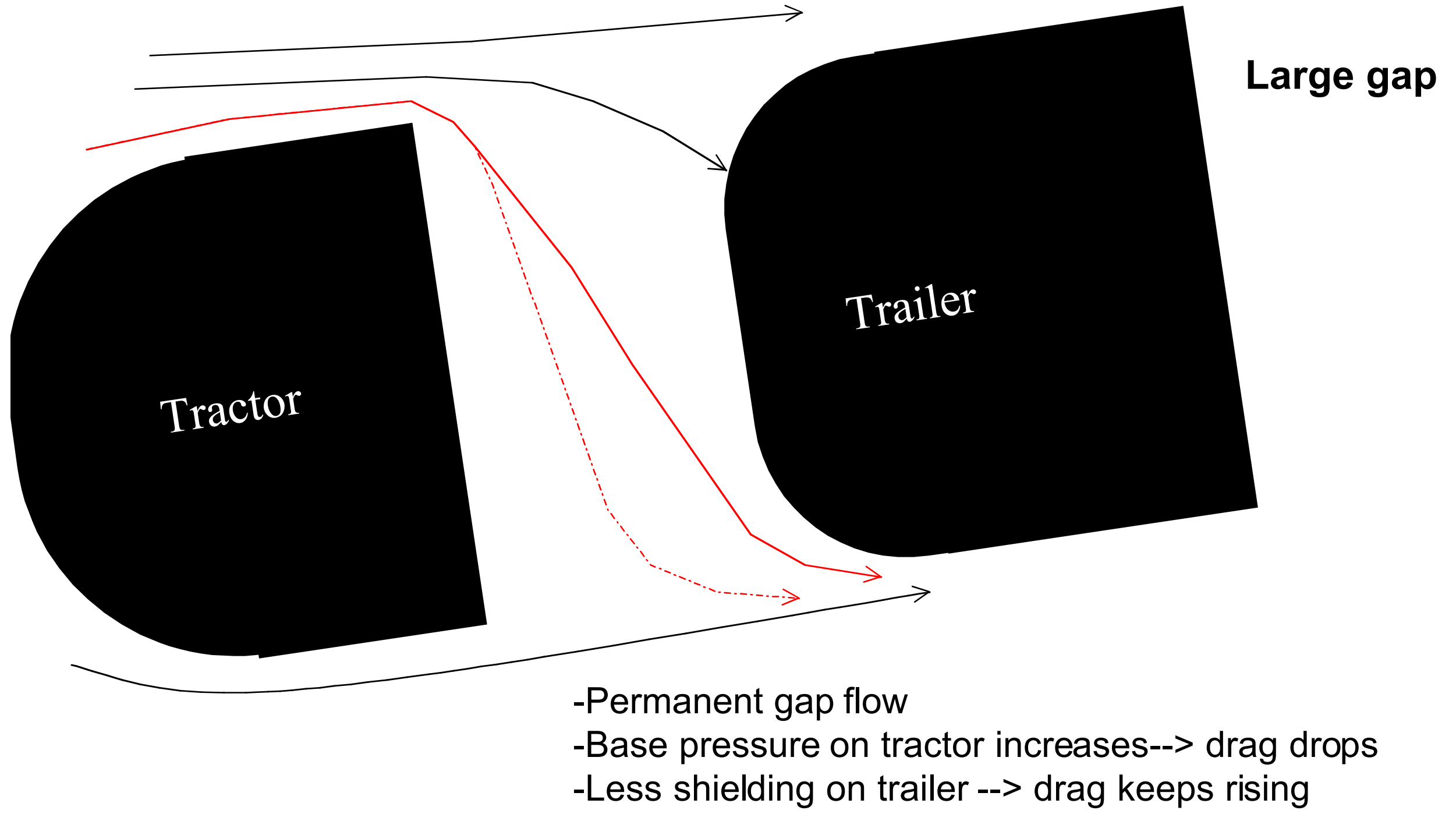


Aerospace Engineering

Ground Vehicle Aerodynamics

\section{ACTIVE BOUNDARY LAYER CONTROL RESEARCH}

\section{PROJECT OBJECTIVES}

- Reduction of drag exerted on a bluff body.

- Identification of energy savings as a function of forcing parameters: amplitude and frequency.

- Comparison of $\left\langle\mathrm{c}_{\mu}\right\rangle$ to other energy saving mechanisms; i.e. $c_{\mu}$ or passive devices.

- Development of a system which can be efficiently applied at full scale. 


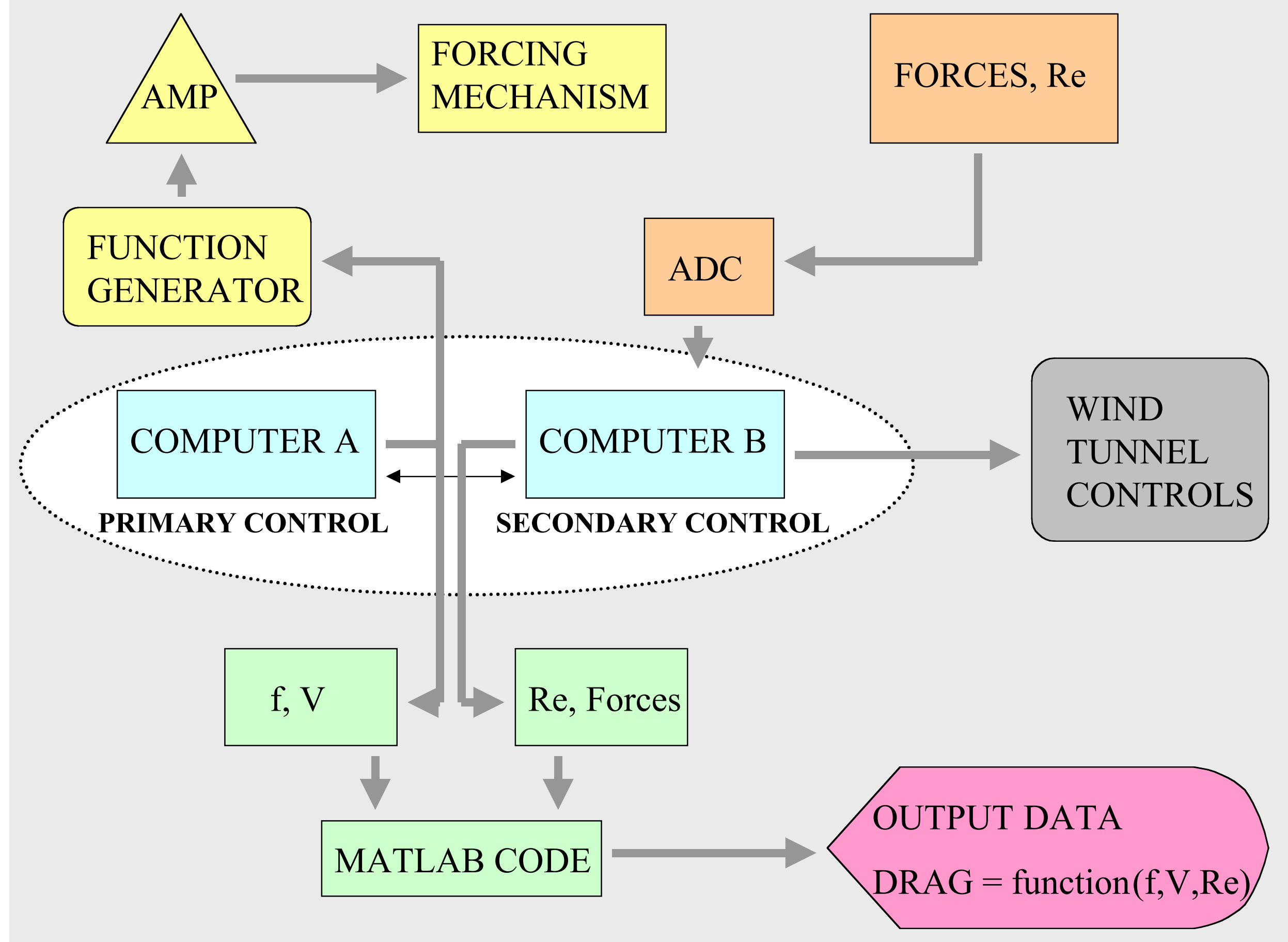




\section{CURRENT RESEARCH}

- Use of thermal anemometry to determine jet exit velocities, $\left\langle\mathrm{c}_{\mu}\right\rangle$.

- Flow diagnostics under consideration - PIV and base pressure measurements.

- Development of new data acquisition procedure. Drag is to be determined as a function of forcing parameters, flow parameters, and model geometry.

- Measure the energy input versus the energy savings for variations in forcing and geometrical arrangements. 


\title{
Computational Prediction for a Simplified Truck Geometry
}

\author{
Kambiz Salari \\ Mary McWherter-Payne \\ Walter H. Rutledge \\ Aerosciences and Compressible Fluid Mechanics Department \\ Sandia National Laboratories \\ Heavy Vehicle Aerodynamic Drag: Working Group Meeting \\ Lawrence Livermore National Laboratory
}

March 29, 2001 


\section{Outline}

- ASCI Overview

- $V \& V$ issues

- Overview of Sandia Accomplishments

- Sandia FY01 Tasks and Budget

- Current status of activities

- Grid Patching issues

- Predictive Capability of RANS

- Status of DES

- Added visualization capability for unsteady flow simulations (with Bob Haimes, MIT)

- Conclusions 


\section{Mission}

Provide trusted engineering analysis codes to the nuclear weapon complex.

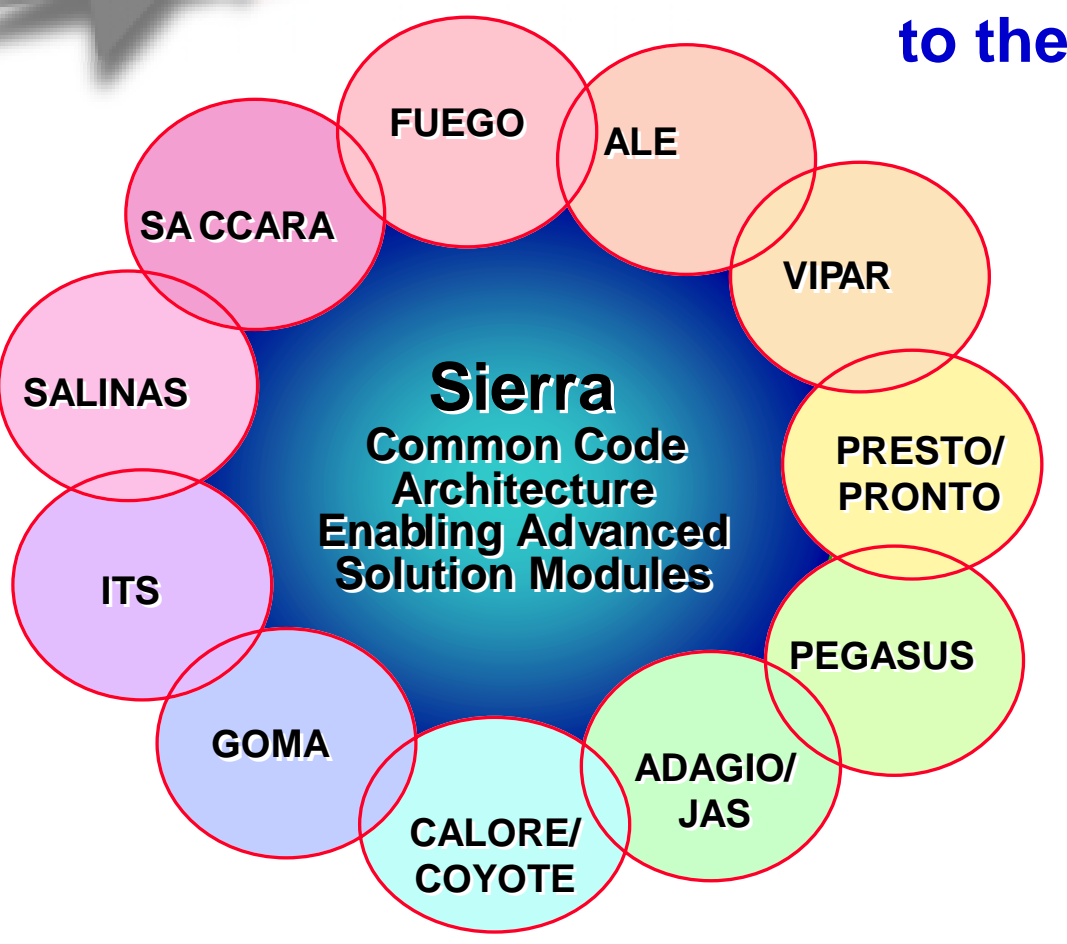

Finite-element services shared among codes

- software quality support

- extensive finite element library

- adaptive meshing

- fully parallel on NWC computers

- dynamic load balancing

- linear solvers

- code coupling

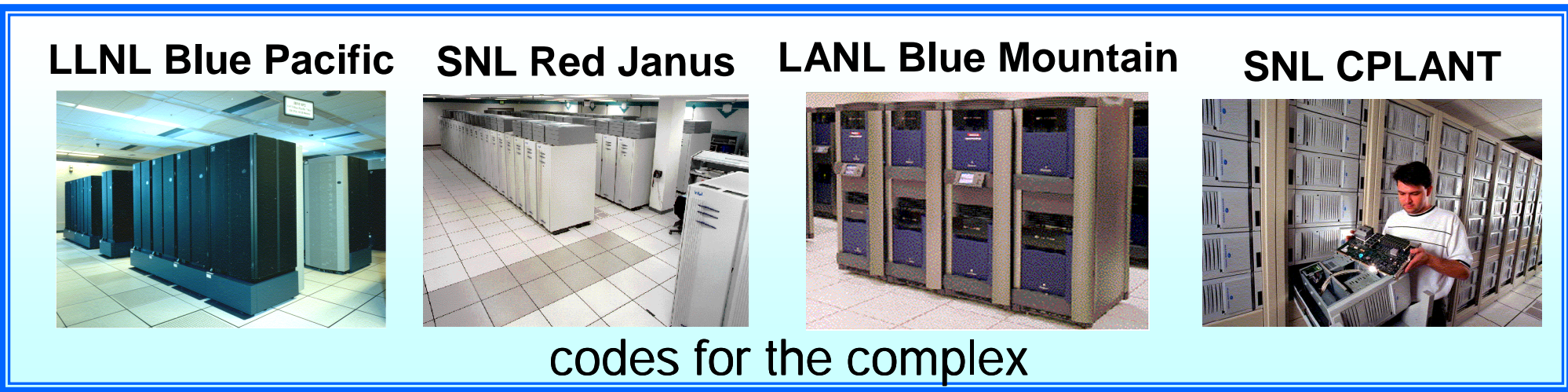




\section{SACCARA Code Capabilities}

$\underline{\text { Sandia }} \underline{\text { Advanced }} \underline{\text { Code for }} \underline{\text { Compressible }} \underline{\text { Aerothermodynamics }} \underline{\text { Research and }} \underline{\text { Analysis }}$

- Multi-block, structured grids for 2-D, Axisymmetric, and 3-D flows

- Solution of the Full Navier-Stokes equations for compressible Flows (RANS/DES/LES)

- Finite volume spatial discretization (steady and unsteady)

- MP implementation on a variety of distributed parallel architectures (IBM, Intel, etc.)

- Implicit time advancement schemes

- Subsonic $\rightarrow$ Hypersonic flows

- Zero-, one-, and two-equation turbulence models

- Ideal, equilibrium, and thermo-chemical nonequilibrium finite-rate gas chemistry

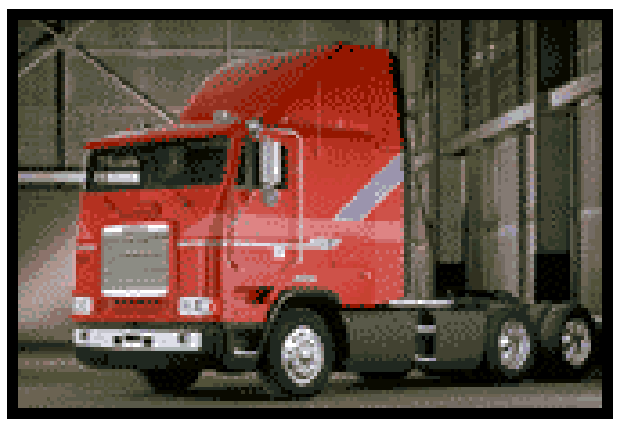

Ablation boundary conditions

- Rotating coordinate system

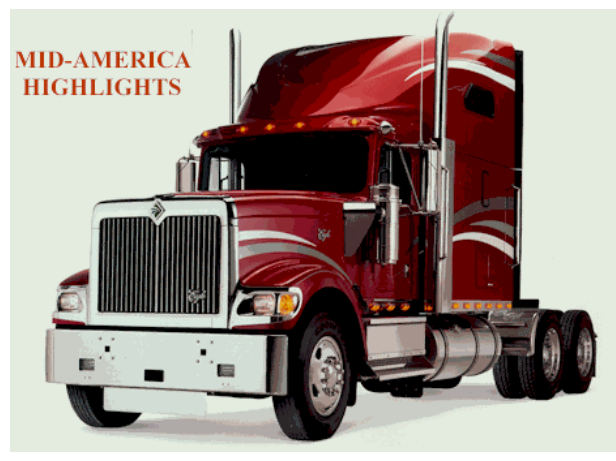




\section{ASCI Program Elements}

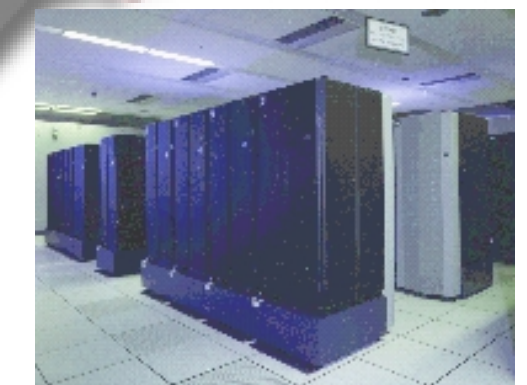

\section{Computers}

Develop massively parallel, high performance computers to achieve the ASCI 100 TeraOps computing goal by 2004

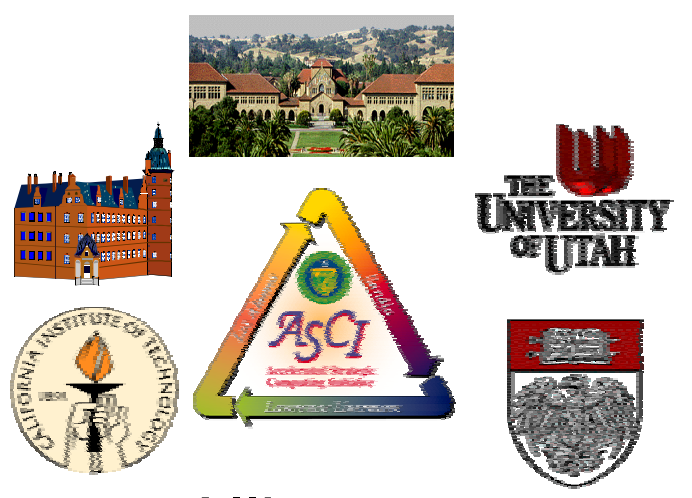

Alliances

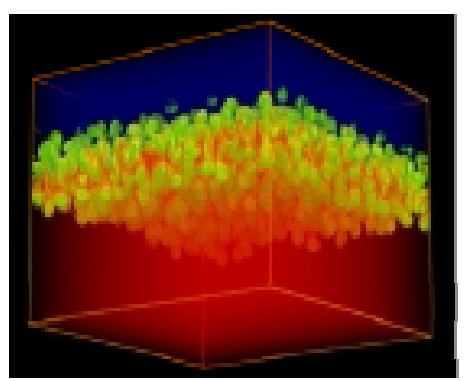

\section{Applications Codes}

Modify and develop codes to achieve the speedup's and improvements necessary to perform full physics simulation

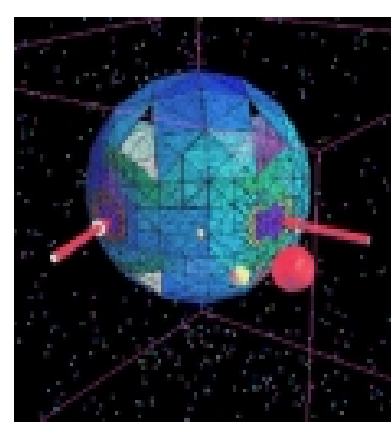

Make the full-physics codes user friendly to weapons analysts including set-up of large scale problems, transferring and storing tera-byte size files, and $3 \mathrm{D}$ visualization on the desktop of these terabyte size files

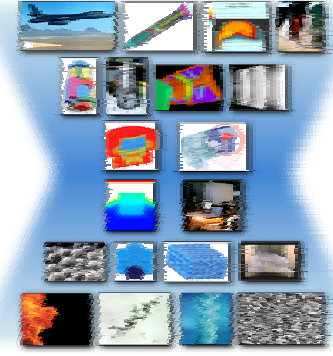

Verification and

Validation

- Provide the tools methodologies and data to ensure that high-end simulation capabilities reflect reality

- Establish confidence in the predictive capabilities of ASCI tools

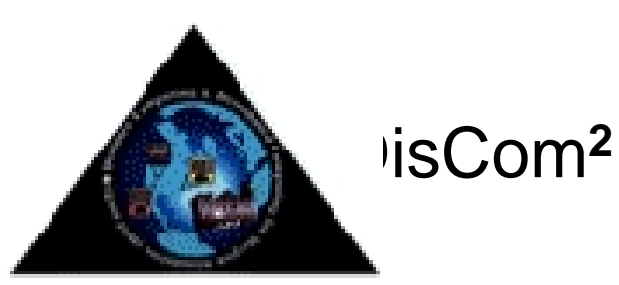

- Implements secure Tera-scale computing across 1000 s of miles

- Integrate information and simulation

- Install distributed security and resource management

- Demonstrate high capacity distributed computational plant 


\section{ASCI Red Platform}

ASCI INTEL “RED” TeraOp Computer Sandia National Laboratories 1.8 TeraOps

Tightly Coupled Distributed Memory HP Interconnect, 9000 200MHz Processors 2 processors/node MPI Programming Model 600 Gbytes Memory

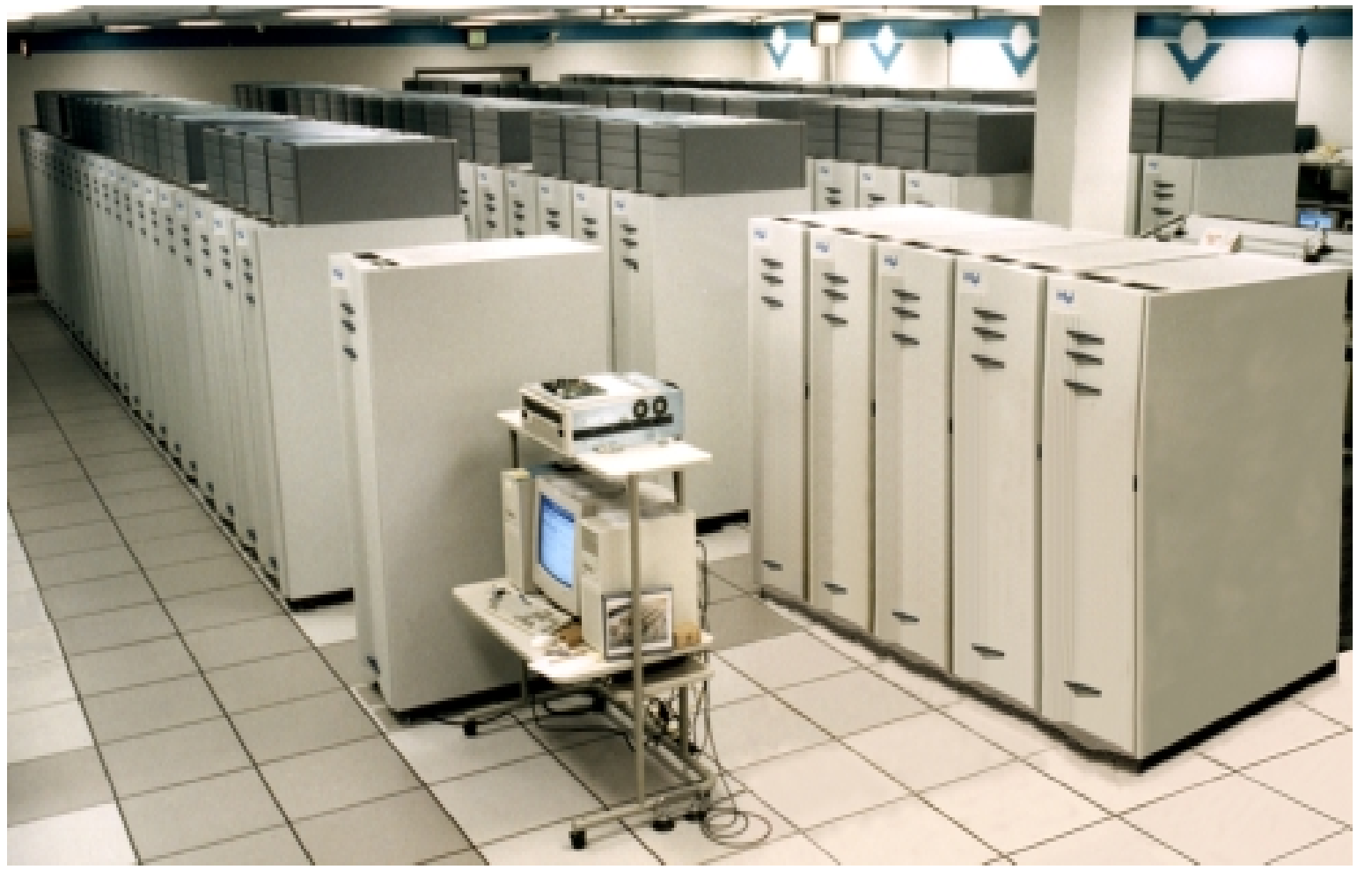

ASCI Red is:

- Providing outstanding performance and stability

- Transitioned to a production mode

- Doing important work for the Stockpile Stewardship program

Effectively saturated 


\section{Understanding the Solution}

- Because of the influence from the ASCI program, Sandia is very concerned about the following issues (and these are big issues!):

- Verification

- Validation

- Uncertainty Quantification, including:

- Solution Accuracy Assessment

- Grid Resolution Studies

- Sensitivity to input parameters

- Other sources of error or uncertainty

- AIAA 2000-2549 paper provides further guidance on V\&V and uncertainty quantification for CFD 


\section{What is a Validation Experiment?}

Based on: "Guide for the Verification and Validation of Computational Fluid Dynamics Simulations" (AIAA-G-077-1998)

1) A validation experiment should be jointly designed and executed by experimentalists and code developers.

- Teamwork and candor are essential

2) A validation experiment should be designed to capture the relevant physics, all initial and boundary conditions, and auxiliary data.

- Leave no loop-holes in the comparison

3) A validation experiment should utilize any inherent synergisms between experiment and computational approaches.

- Offset strengths and weaknesses of each approach 


\section{What is a Validation Experiment? (cont'd)}

4) The flavor of a blind comparison of computational results with experimental data should be a goal.

- It should be a "true prediction," not a calibration

5) A hierarchy of complexity of physics should be attacked in a series of validation experiments.

- Identify levels of complexity and difficulty of prediction

6) Develop and employ experimental uncertainty analysis procedures to delineate and quantify systematic and random sources of error.

- Use symmetry arguments to help identify systematic errors

- Validation experiments are new types of experiments. 


\section{Levels of Validation}

- Increasing levels of complexity.... (applied to both experiments and computations)

- Unit Level

- Benchmark Level

- Sub-System Level

- System Level

- Refer to AIAA-G-077-1998 for more clarification on Validation Levels 


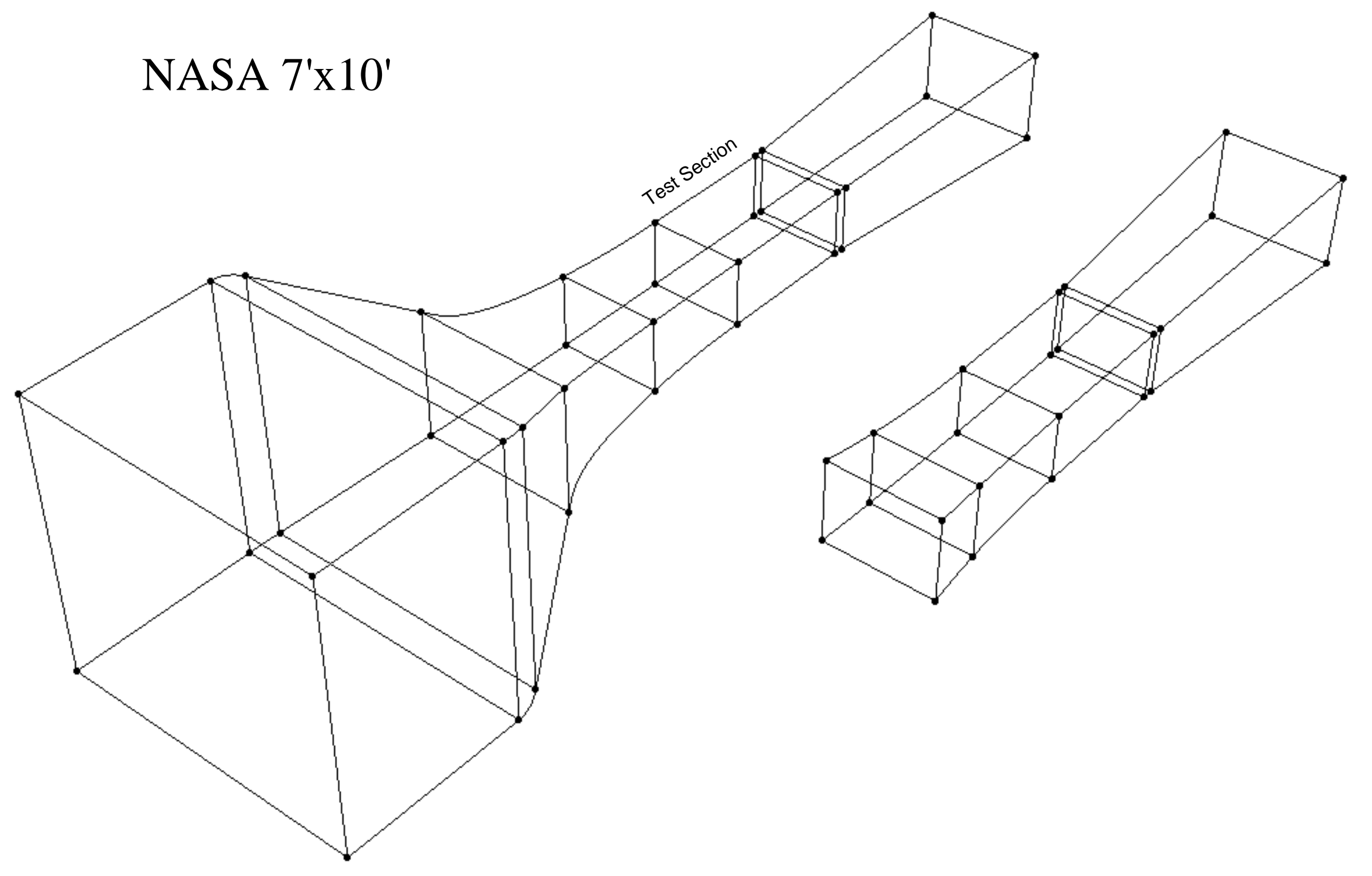




\section{NASA 7'x10' Tunnel, Flow Simulation}

Pressure contours

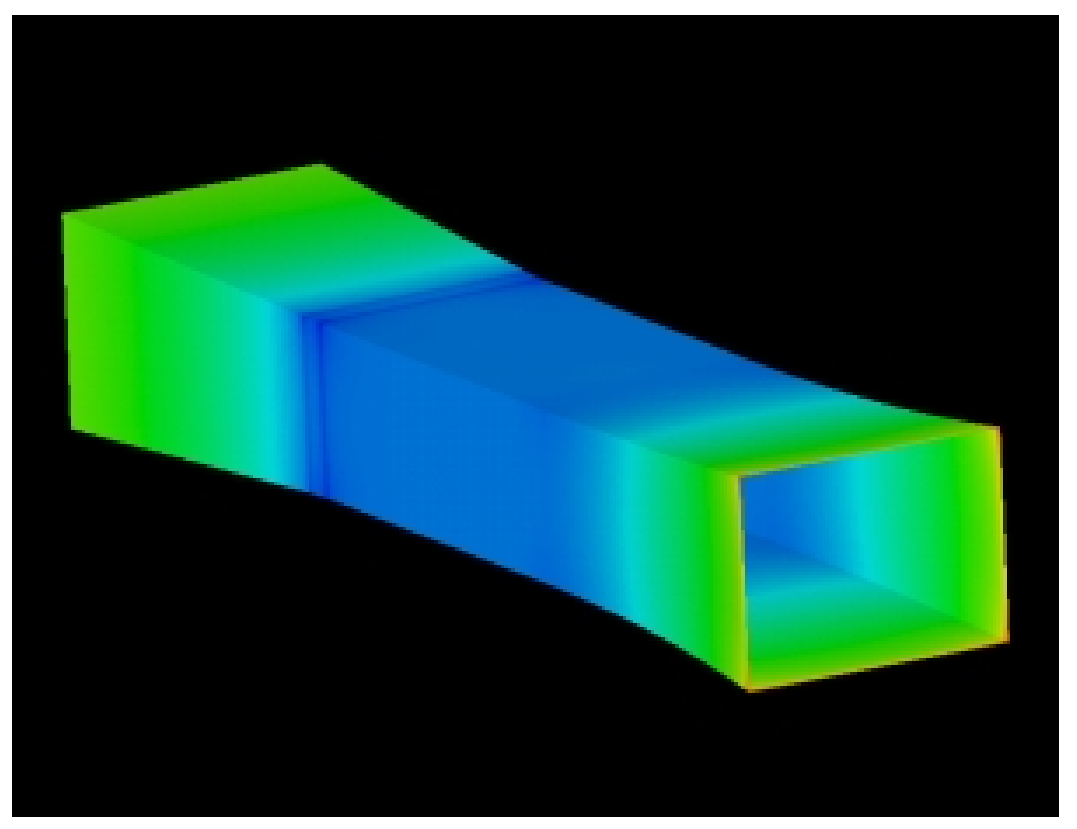

Mach contours (centerline)

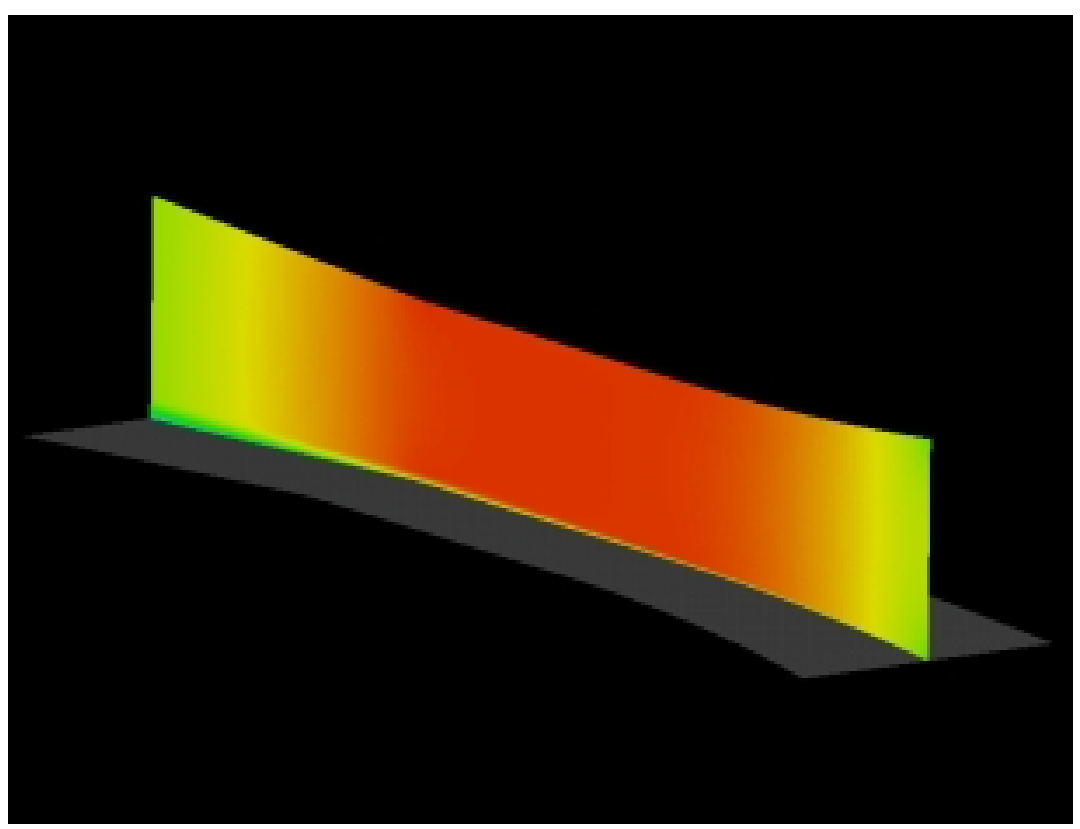




\section{Steady RANS Solution, $0^{\circ}$ yaw}

Mach contours (centerline)

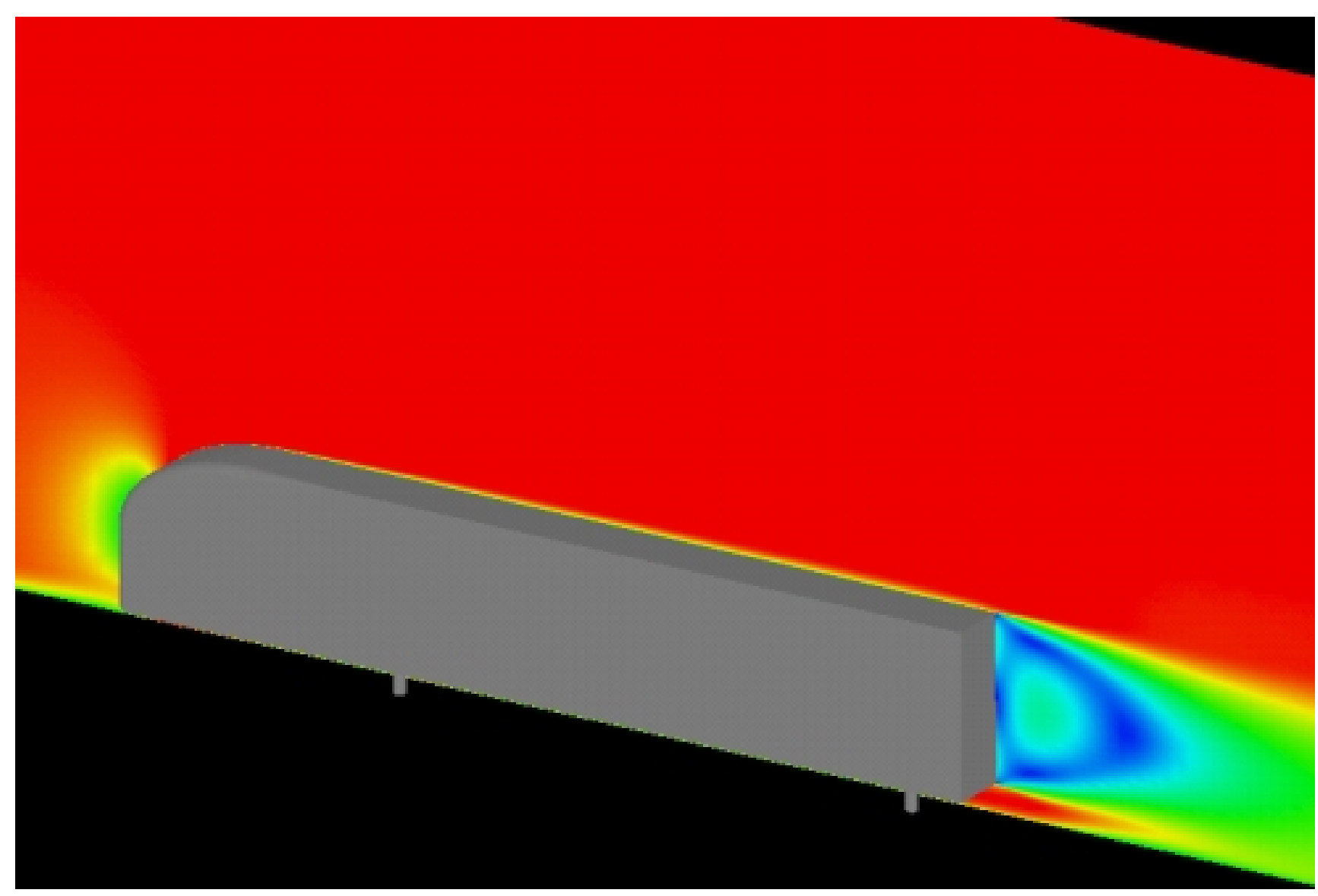




\title{
GTS Flow Simulation, 10 yaw
}

\author{
$10^{\circ}$ yaw \\ y-plane cut \\ Mach contours
}

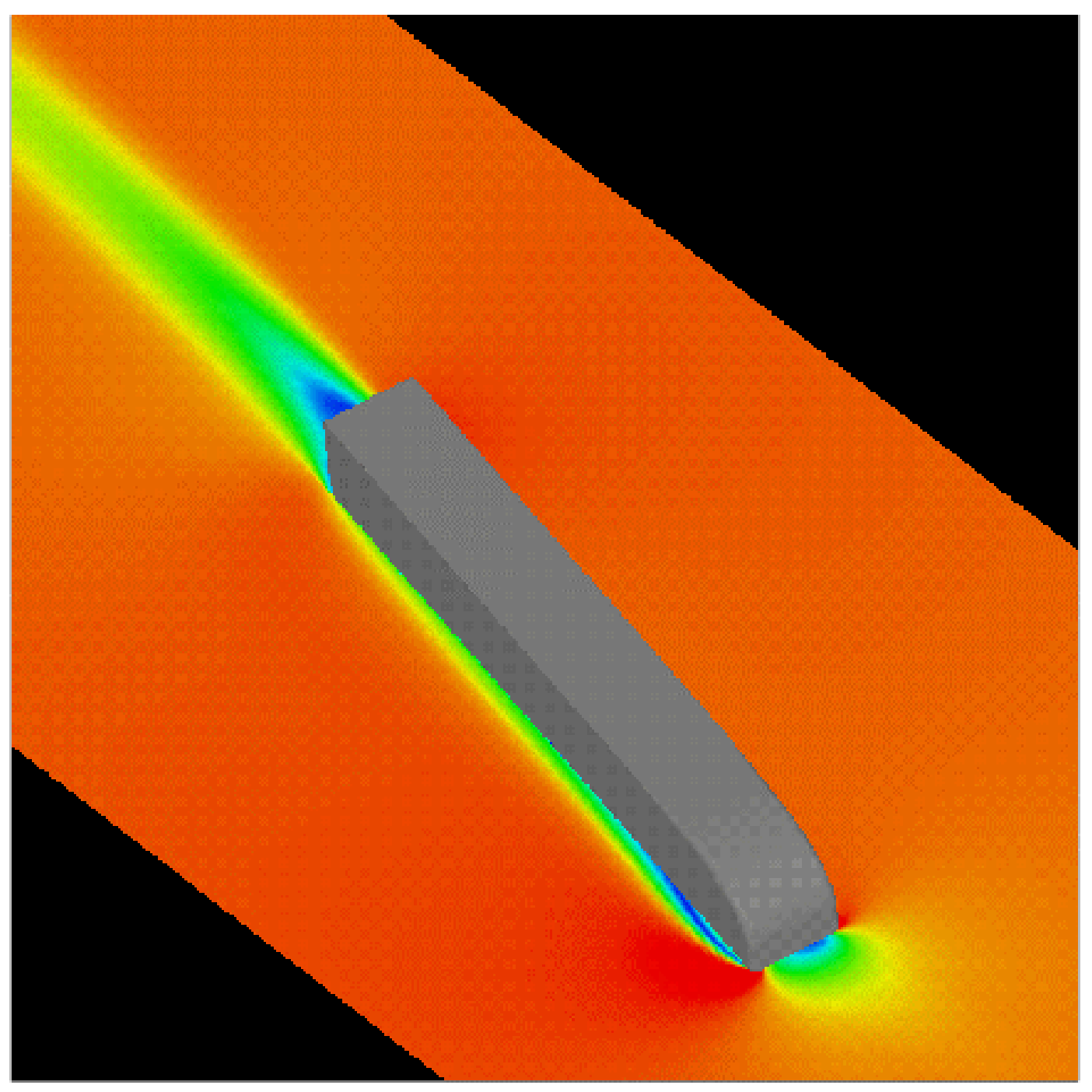




\section{Separation Bubble, Leeward Side, $10^{\circ}$ yaw}

Iso-Surface $\mathbf{u}=\mathbf{- 0 . 0 0 1}(\mathrm{m} / \mathrm{s})$
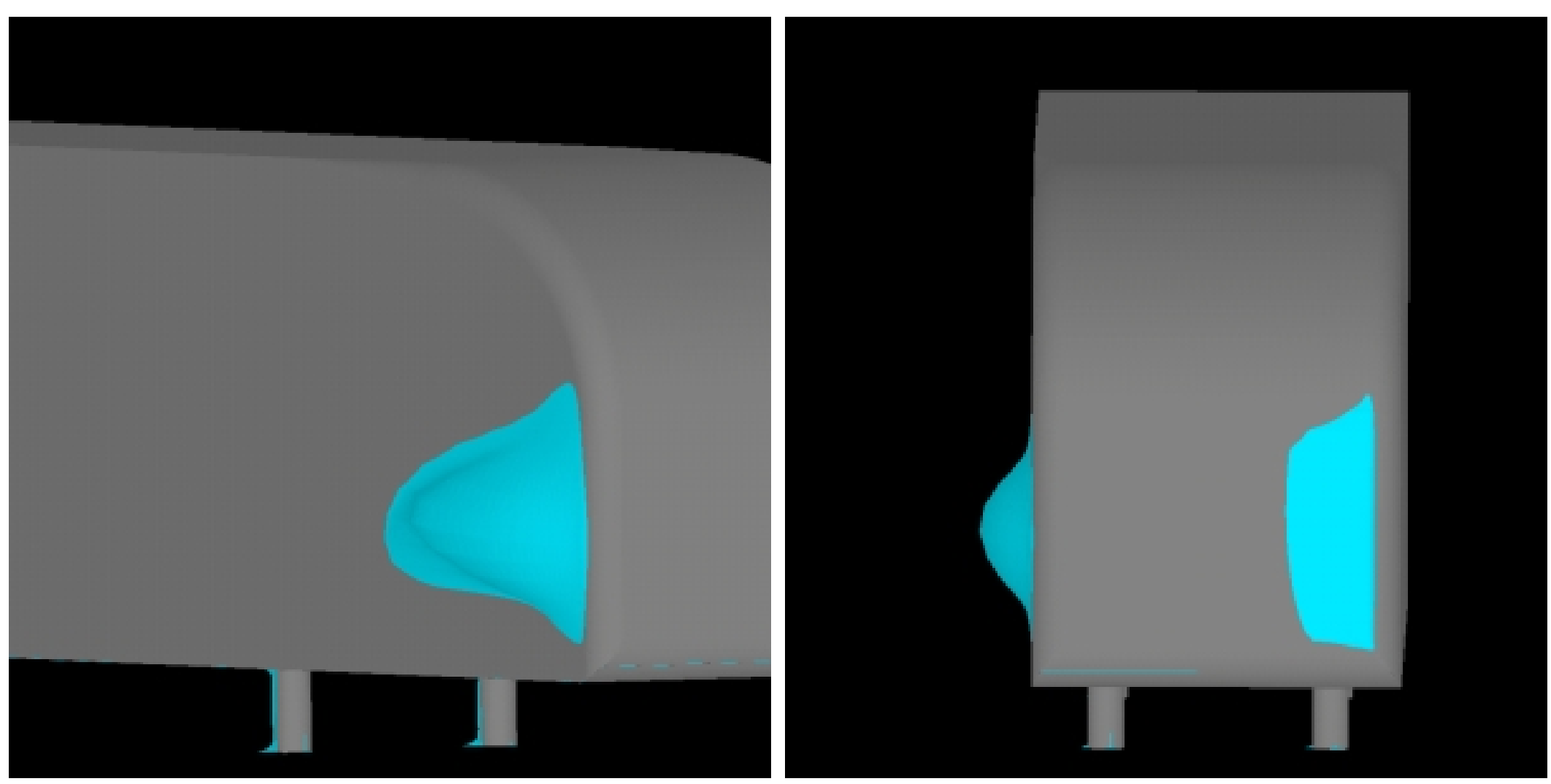


\section{Hot Film Results}

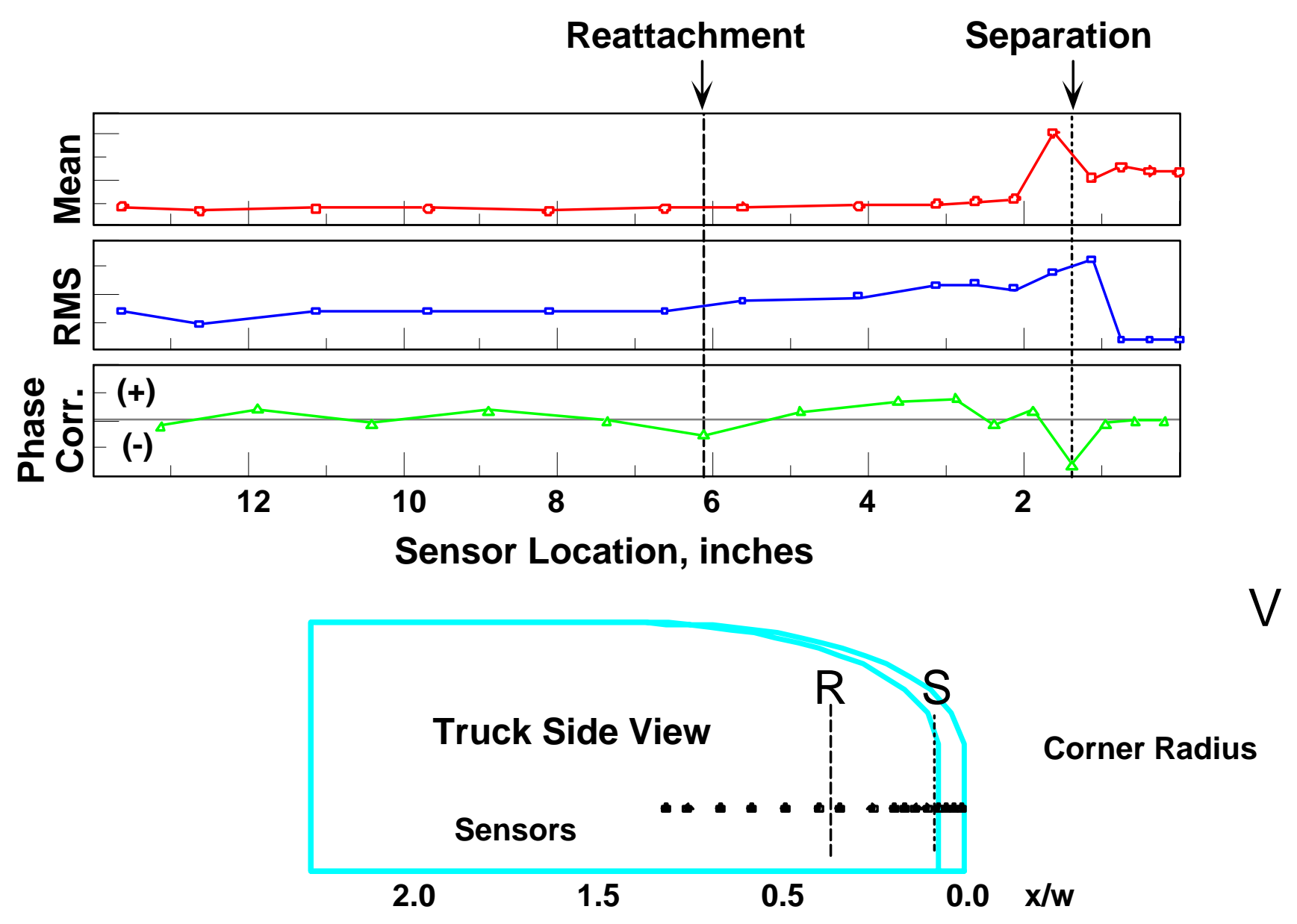




\section{GTS Flow Simulation, DES Result}

Contours of turbulent viscosity, $x y$ and $y z$ cutting planes
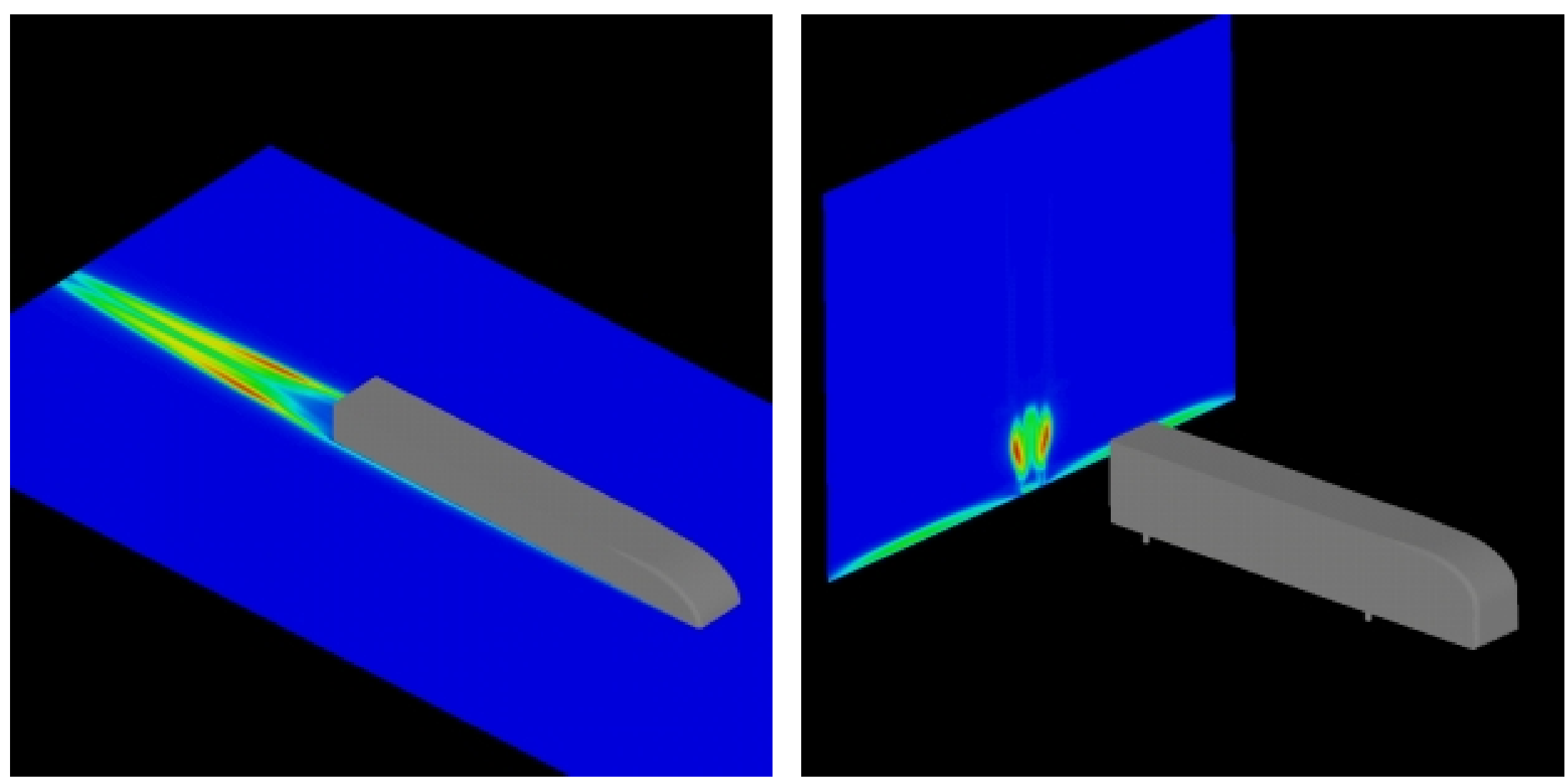


\section{Sandia Tasks for FY01}

1. Complete March 2002 SAE paper that documents RANS solutions for $0^{\circ}$ and $10^{\circ}$ yaw angles (NASA $7^{\prime} \times 10^{\prime}$ )

2. Perform grid resolution for RANS calculations

3. Construct grids for DES simulations

4. Perform DES calculation $0^{\circ}$ yaw angle

5. Document the DES results in a March 2002 SAE paper

6. Investigate the capability of RANS eddy viscosity models (SA, $k-\varepsilon, k-\omega, k-\zeta)$ to predict the wake flow of the GTS and document in a SAE paper (this task is very important for evaluating accuracy of RANS)

7. Help design and participate in future NASA wind tunnel experiments (task currently unfunded)

8. Improve visualization for unsteady flow simulations (Real-Time feature extraction for RANS/DES/LES) 


\section{Sandia Funding Status}

- Funding request for all tasks was \$330K (Aug. 00)

- FY01 Project Funding Decision: \$225K

- All funding has been received

- Year-to-Date Spending: \$69K

- All tasks (except Task 7) expected to be completed this year (\$285K tasks accomplished for $\$ 225 \mathrm{~K}$ )

- Leveraging

- Eng. Sci. Research Foundation funding for DES research

- ASCI Sub-Grid Physics funding:

- RANS Turbulence Modeling

- ASCI Code Development, University Alliance funding for boundary layer transition research

- ASCI Red Teraflop Computer (9000 processors) 


\section{Current status of activities}

- GRIDGEN - new version of GRIDGEN has a bug in providing block patching information for SACCARA and had to revert back to older version

- SACCARA - have uncovered a bug in the $k-\omega$ turbulence model (now fixed)

- DECOMP - This code provides decomposition of the grid for parallel runs. Uncovered a bug for the inter-zone patching

- Janus - Lack of availability since December due to $0 S /$ hardware stability issues (this has severely affected our productivity) 


\section{Boundary Condition: Inter-Zone Patch}
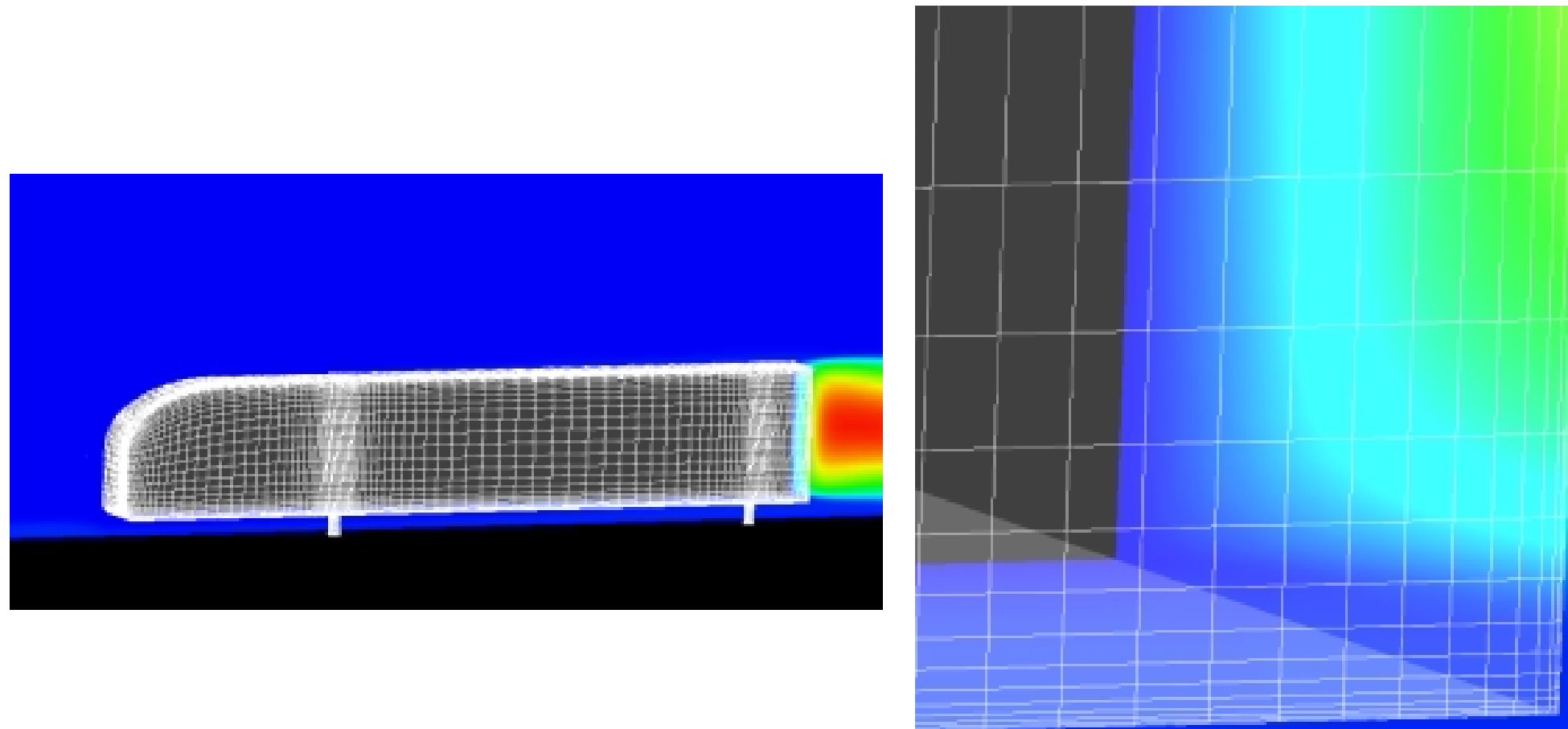


\section{RANS Solutions}

- Simulate truck model in the tunnel for "validation exercises"

- Good agreement over the entire truck except in the base region

- RANS code appears to be useful for design tradeoff studies (but not absolute numbers)

- RANS code (at least with one-equation SA model) does not appear to capture the right wake structure

- New FY01 task will assess newer and more advanced turbulence modeling based on eddy viscosity models (e.g., $k-\omega, k-\zeta)$ 


\section{Steady RANS Solution, $0^{\circ}$ yaw}
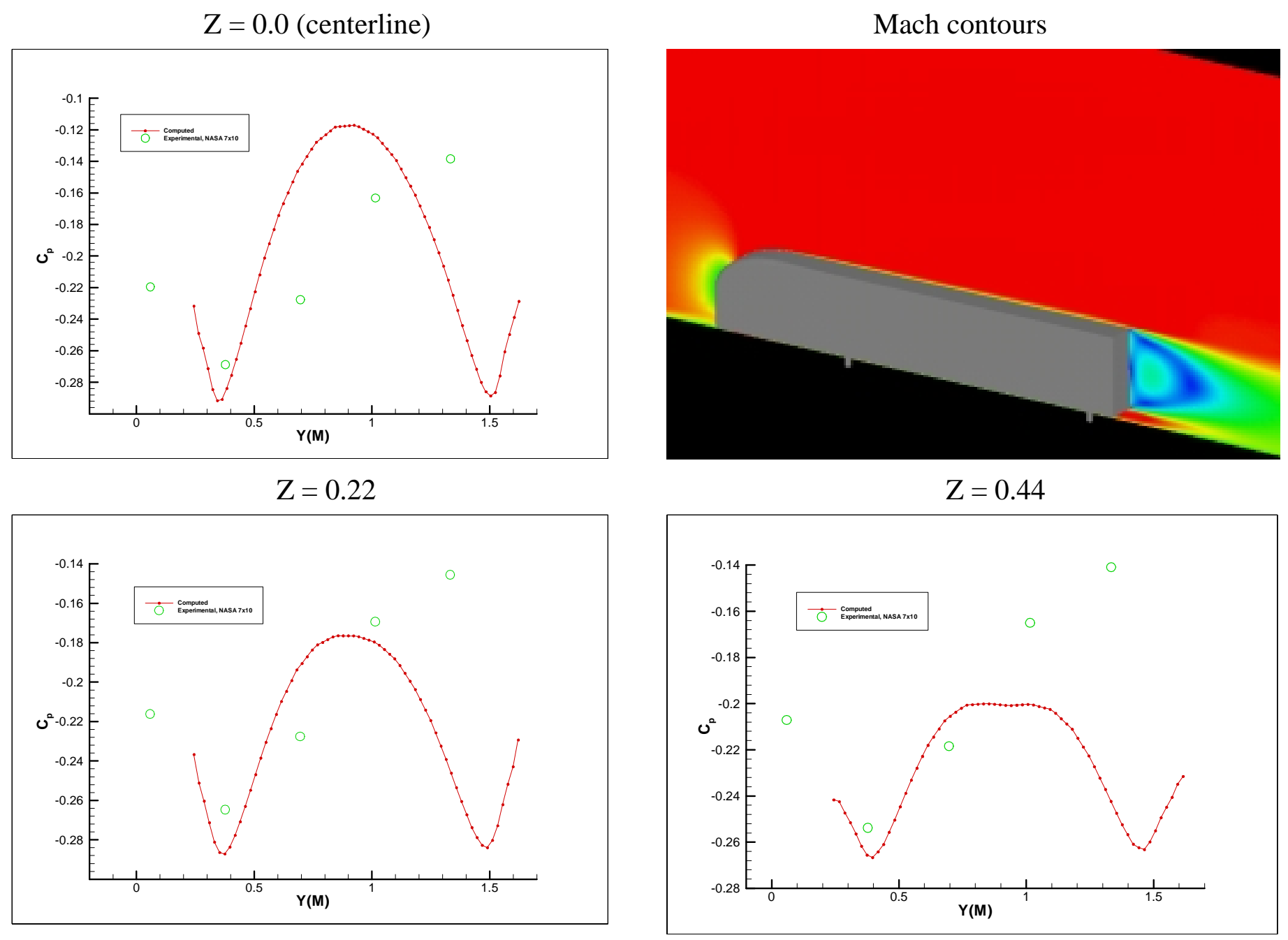


\section{Steady RANS Results , $0^{\circ}$ yaw, ...}
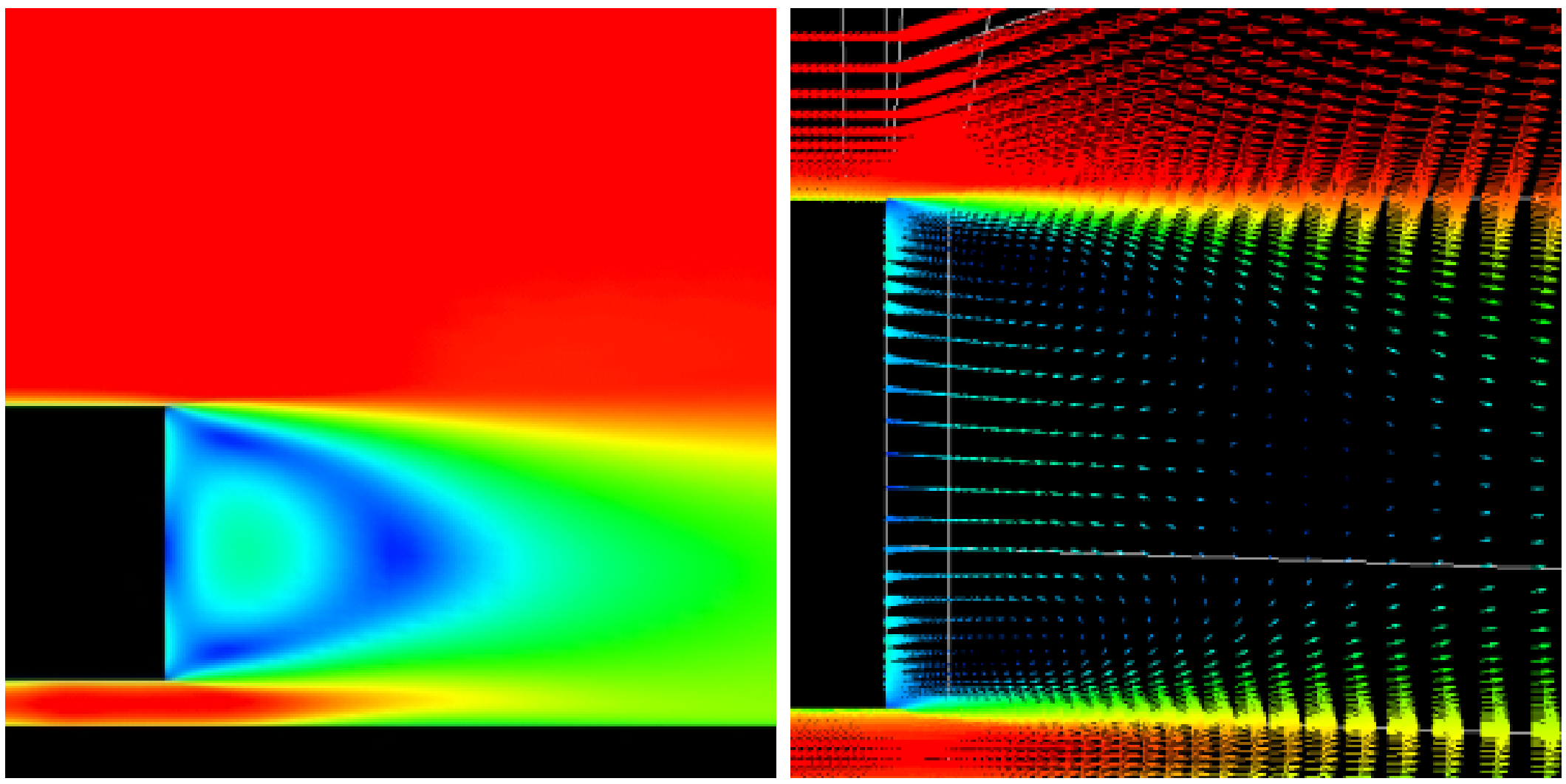


\section{Status of DES}

- DES simulation of the GTS model in the NASA 7'x10' tunnel that has been on going is on hold because of the block and inter-zone patching and boundary condition issues (hope to resume in April)
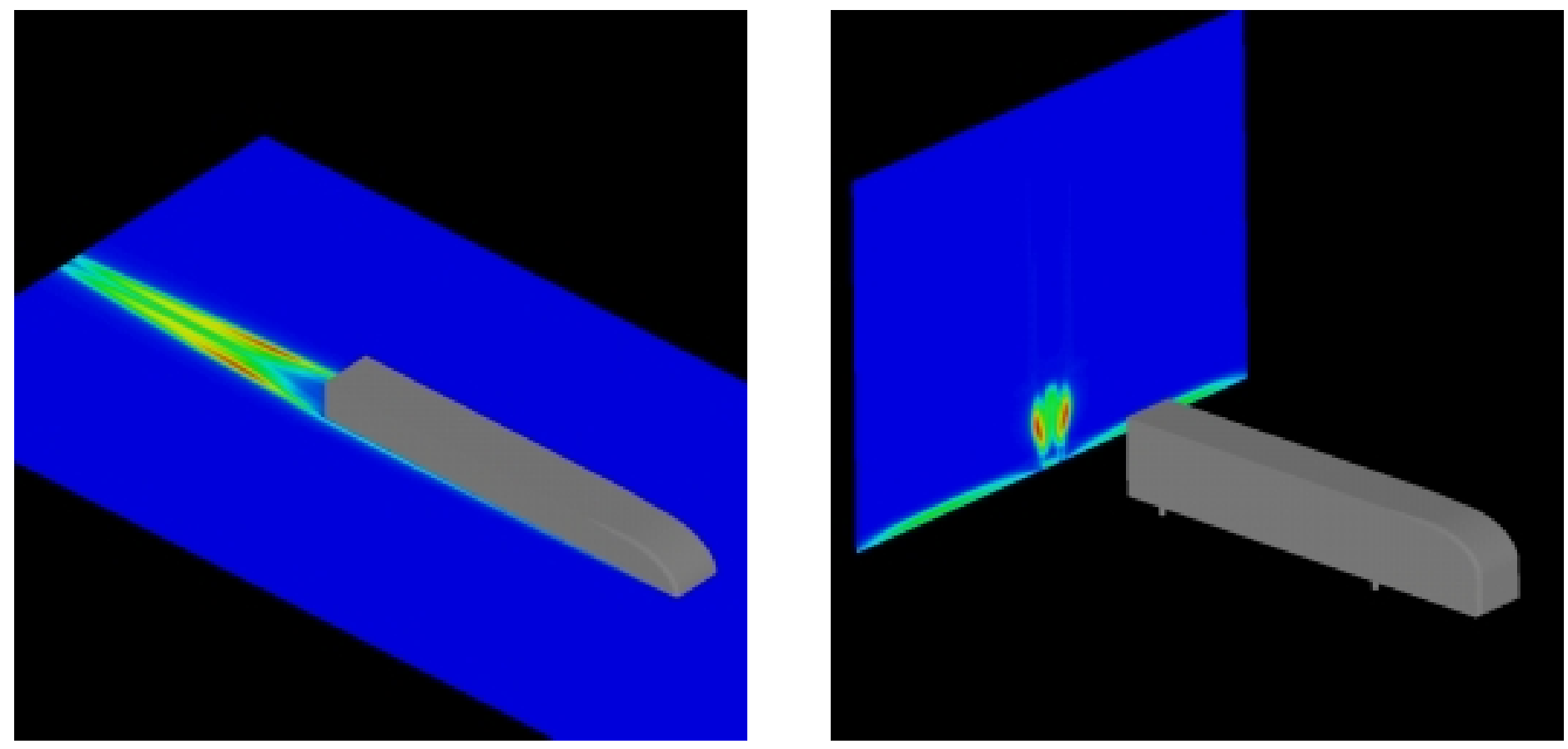


\section{Added Capability to Visualize Large Dataset}

- DES/LES unsteady simulations produce very large datasets

- Feature extraction, real-time on MP machine (vs. postprocessing)

- Vortex core

- Surface cutting plane

- Particle traces

- Separation and reattachment lines

- Significant reduction in storage

- For the circular cylinder coarse grid example, $250 \mathrm{MB}$ of storage was required as opposed to $20 \mathrm{~GB}$ of storage for the entire volume to be stored for proper post-processing 


\section{Movie time}




\section{Concluding Remarks}

- Due to the uncovered bugs, all the calculations that have been performed so far are suspect and need to be investigated for the sensitivity of the solutions to the inter-zone patch difficulties (ongoing)

- Documentation of previous RANS simulations are on hold until we have a better understanding of the impact of the patching issues on these solutions (should pick back up in April) 


\section{Concluding Remarks, ...}

- The uncovered bugs in block patching and interzone patching have been corrected

- All previous grids for GTS model have been modified to eliminate the inter-zone patching problem

- Currently, we are rerunning our calculations with different turbulence models with the above corrections

- All scheduled tasks should be completed and documented by the end of FY01 


\title{
Simulation of Complex, Unsteady Flows Using a Grid-Free Vortex Method
}

\author{
A. Leonard, P. Chatelain, M. Rubel \\ Graduate Aeronautical Laboratories \\ California Institute of Technology
}

March 28-29, 2001 


\section{Vortex Code: Essentials}

- Numerical technique to solve the Navier-Stokes Equations

- Suitable for Direct Simulation and Large-Eddy Simulation

- Uses vorticity (curl of the velocity) as a variable

- Computational elements move with the fluid velocity 


\section{Vortex Code: Advantages}

- Computational elements only where vorticity is non-zero

- No grid in the flow field

- Only 2D grid on vehicle surface

- Boundary conditions in the far field automatically satisfied 


\section{Large-Eddy Simulation}

\section{Direct Simulation not Sufficient (1990s)}

- Direct Simulation possible for Reynolds no. $=10^{3}$ to $10^{4}$ (at parking speeds $-0.01 \mathrm{mph}$ )

- $N=10^{12}$ elements (approx. 20 Terabytes) required for Reynolds no. $=5 \times 10^{6}$ (at highway speeds)

\section{Large-Eddy Simulation Required}

- Treatment of small-scale (subgrid-scale) turbulence in the wake

- Treatment of small-scale turbulence in the boundary layers

- Treatment of fluidic actuators, blowing/suction, vortex generators and other flow control devices 


\title{
Numeric Time Integration by Dead Reckoning
}

A Tool-In-Progress for Accelerating Heavy-Vehicle Aerodynamic Computation

\author{
Mike Rubel \\ Tony Leonard
}

Graduate Aeronautical Laboratories · California Institute of Technology
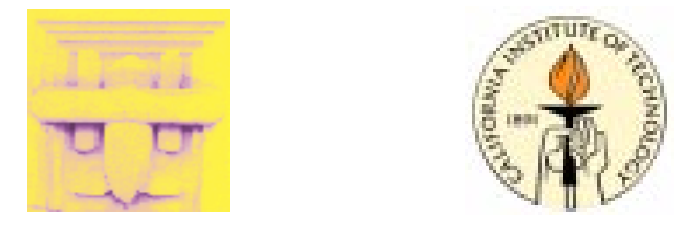

March 29, 2001 


\section{Fully-Resolved DNS of Flow Around a Truck at Re $10^{7}$}

$$
\text { ... just kidding }
$$

- Luckily, we know the equations

- We can even solve them sometimes

- The problem is speed

- Searching for pockets of inefficiency or redundancy

- An observation ... 


\section{Time Integration of the Flow Equations}

Discretizing the flow equations (vorticity equation, in our case) gives a system of the form:

$$
\frac{d X}{d t}=A(X) \quad X(0)=X_{0}
$$

where:

- $\mathrm{X}$ is the state vector

- $A$ is a (dense) nonlinear operator

In vortex particle methods, $\mathrm{X}$ contains the strength and position of each particle and the boundary panel strengths. In finite-difference methods, it contains values of the fields at grid points.

next: integrating the equations numerically 


\section{Timestepping: A Truly Brief Overview}

Solve:

$$
\begin{gathered}
\frac{d X}{d t}=A(X) \quad X(0)=X_{0} \\
X=\left\{x_{0}, x_{1}, \ldots, x_{N}\right\}
\end{gathered}
$$

Take a "small" $\Delta t$, move a "small" $\Delta X$. Small is relative!

$$
\begin{array}{|c|}
\hline \text { Fast } \\
\text { Big Steps }
\end{array}
$$

$\Longleftrightarrow \quad \begin{gathered}\text { Accurate } \\ \text { Small Steps }\end{gathered}$

How do we choose $\Delta t$ when $X$ is a vector with many components? 


\section{The CFL Condition: Astronomical Analogy}

... or, Why We Can't Just Scale Each Variable's Timestep Independently
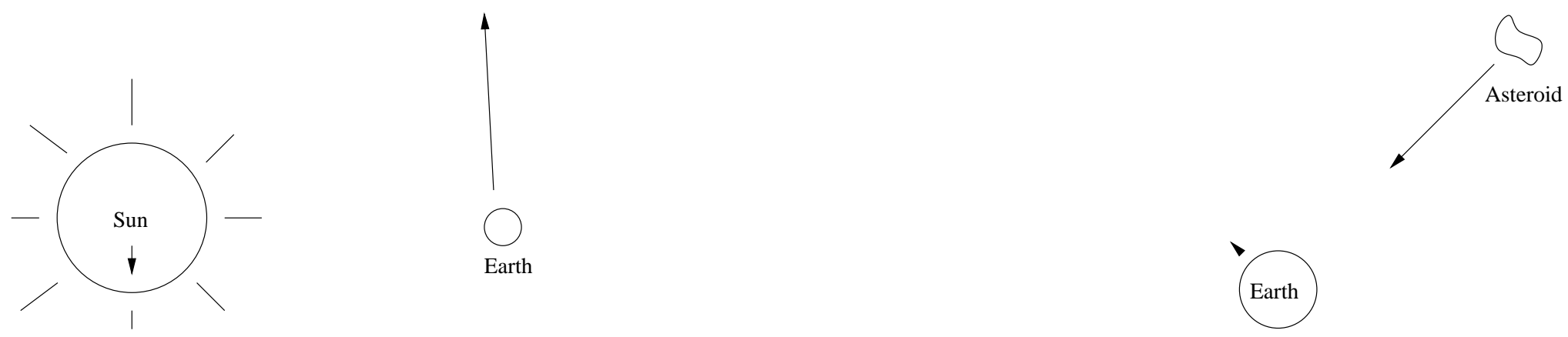

Solution: limit timestep by fastest relative speed, smallest distance $\longrightarrow$ the CFL (Courant, Fredrichs, Lewy) condition.

Next: what does this mean for truck aerodynamics? 


\section{CFL Condition: A Step In The Fluids Direction}

$$
\Delta t_{\max } \propto \min \left(\frac{\sigma^{2}}{\Gamma}, \frac{\sigma^{2}}{\nu}\right)
$$

$\Gamma$ is the largest relative velocity times the largest physical scale in the problem; $\sigma$ is a grid spacing or particle core size.

The effect of using too large a CFL number depends on the timestepper and the problem. Sometimes the calculation becomes inaccurate; sometimes an instability develops.

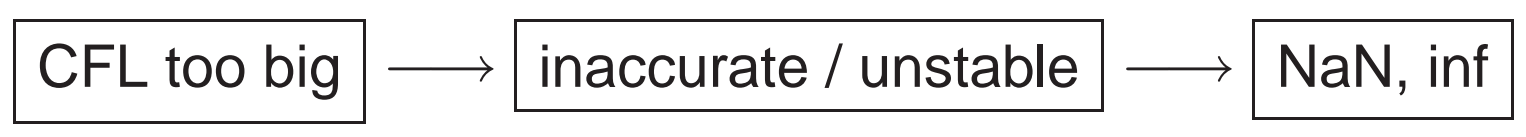

next: how using a single $\Delta t$ leads to redundancy . . . 


\section{Possibly Redundant Computation}

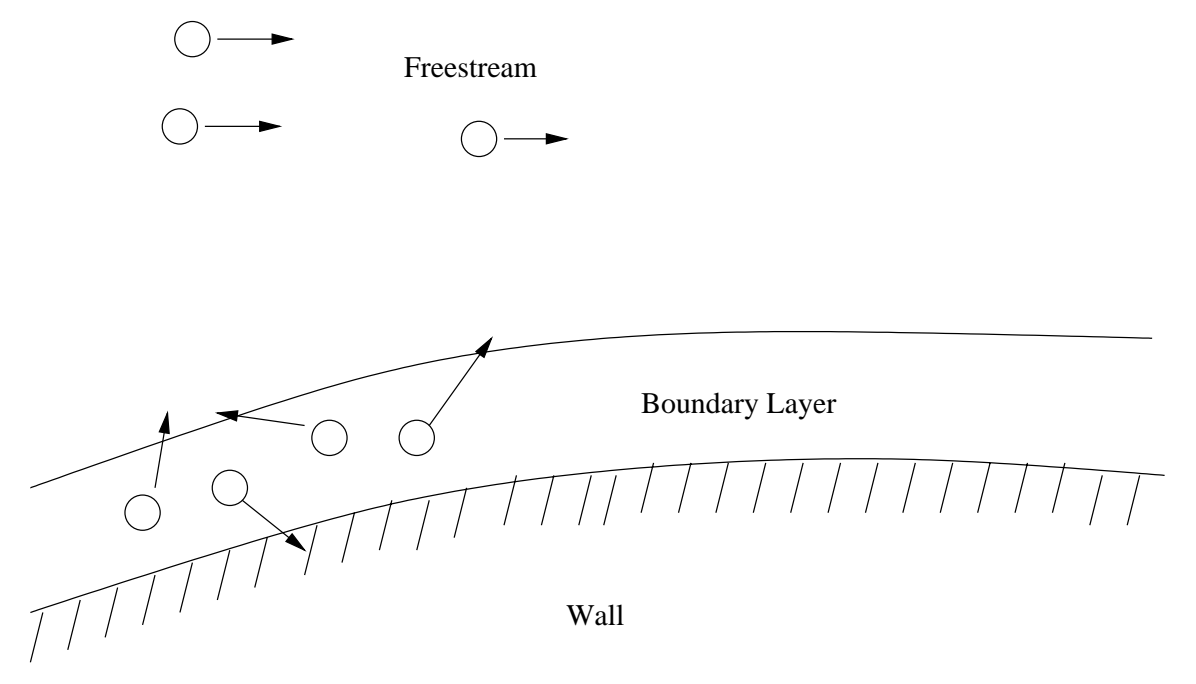

Most of the particles move roughly with the freestream, and they're timestepped very slowly. That's because the largest relative speed controls the time step, and in many flows of interest, there is some small region with a lot of activity. A few particles spoil it for everyone.

next: sometimes we do take different steps 


\section{When May We Use Different Timesteps?}

- Astrophysical simulation

- Bubbles

- Multiple grids

- Chemical reactions

Often problem-dependent. Is there a better way? 


\section{A Slight Detour: Network Games}

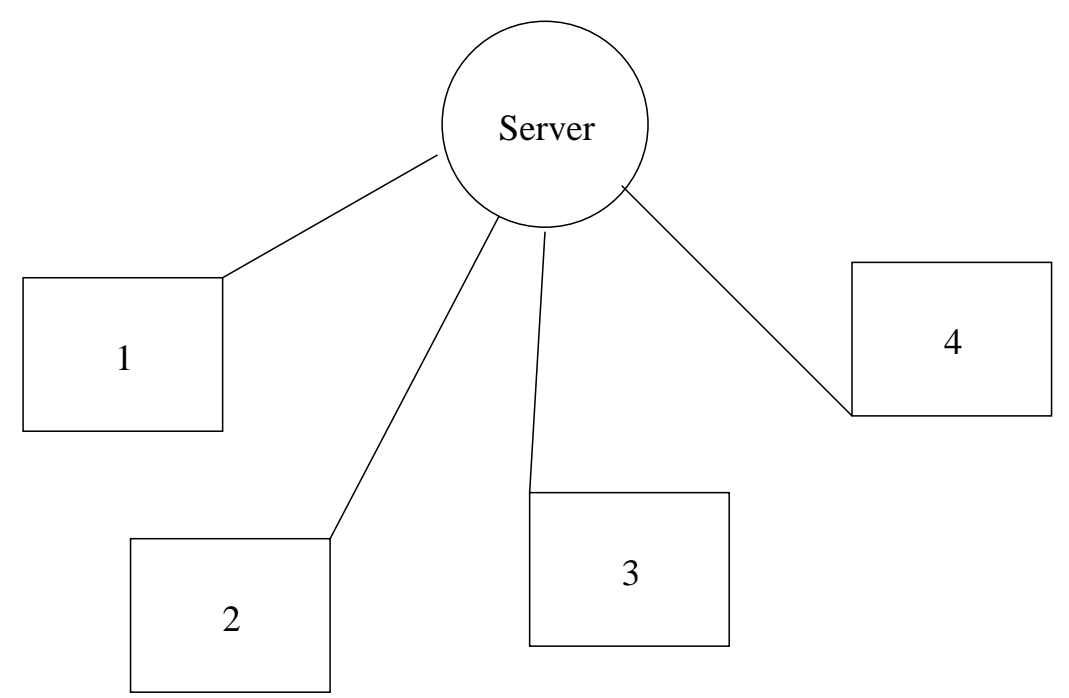

- server insures each version is the same

- reduce the effect of network lag

- use dead reckoning. 


\section{The Dead Reckoning Algorithm}
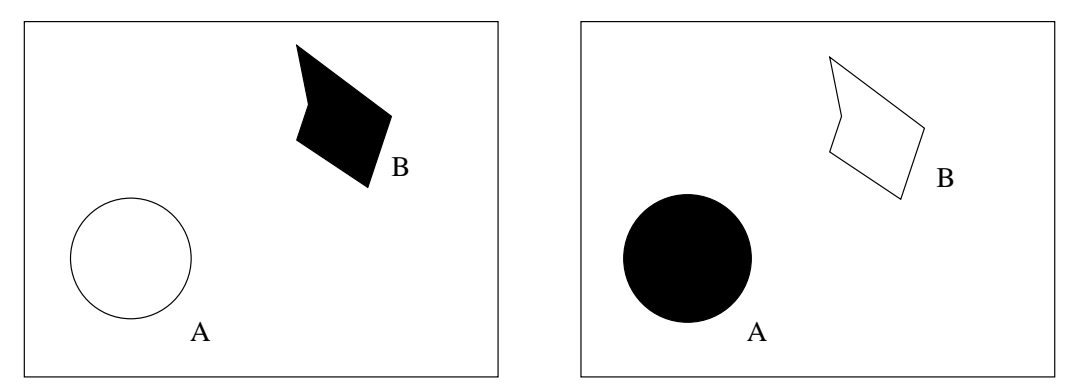

- player A sends position and velocity to player B

- B extrapolates A's position until further notice

- A performs the same extrapolation; sends new position and velocity to $B$ when the error reaches some predefined threshold

next: integration by dead reckoning 


\section{Integration by Dead Reckoning: Algorithm}

Choose a flexible form for each element of $X$ :

$$
X(t)=\left\{x_{1}(t) x_{2}(t) \ldots x_{n}(t)\right\}^{T} \quad x_{i}(t)=a_{i} t+b_{i}
$$

then solve:

$$
Y(t)=\int_{0}^{t} A(X(\tau)) d \tau+X_{0} \quad\|X(t)-Y(t)\|<\epsilon
$$

Assume that at time $t_{0}$, locally-valid coefficients are known for all $x_{i}$. Sleep until one of the following:

- Continue: It is not known how much longer $x_{i}(t)$ will be a valid approximation to $y_{i}(t)$. Timestep forward a small amount; schedule a repost or another continue. Go back to sleep.

- Repost: Revise the coefficients of this $x_{i}(t)$ based on new information. Wake all $x_{j}$ that depend on $x_{i}$ through $A$ immediately with a continue. 


\section{Let's Try An Example}

Solve the (scalar) equation:

$$
\frac{d x}{d t}=2 x \quad x(0)=1
$$

Exact solution $x(t)=\exp (2 t)$. 


\section{Results: a Diagonal System}

$$
\dot{X}=\left[\begin{array}{cc}
1 & 0 \\
0 & -1
\end{array}\right] X \quad X_{0}=\left[\begin{array}{l}
1 \\
1
\end{array}\right]
$$

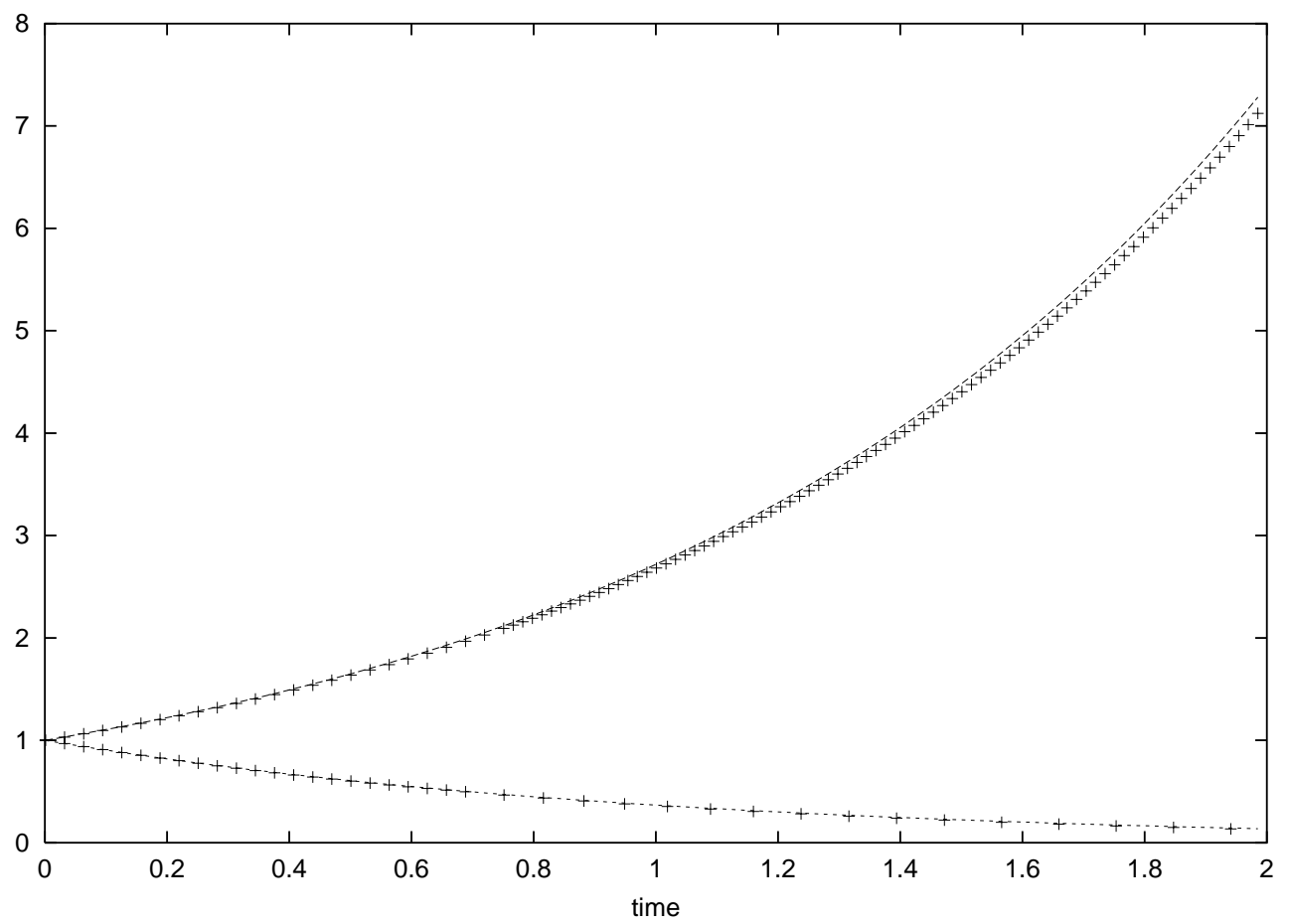




\section{Results: a Not-Diagonal System}

$$
\dot{X}=\left[\begin{array}{cc}
0 & 1 \\
-1 & 0
\end{array}\right] X \quad X(0)=\left[\begin{array}{l}
0 \\
1
\end{array}\right]
$$

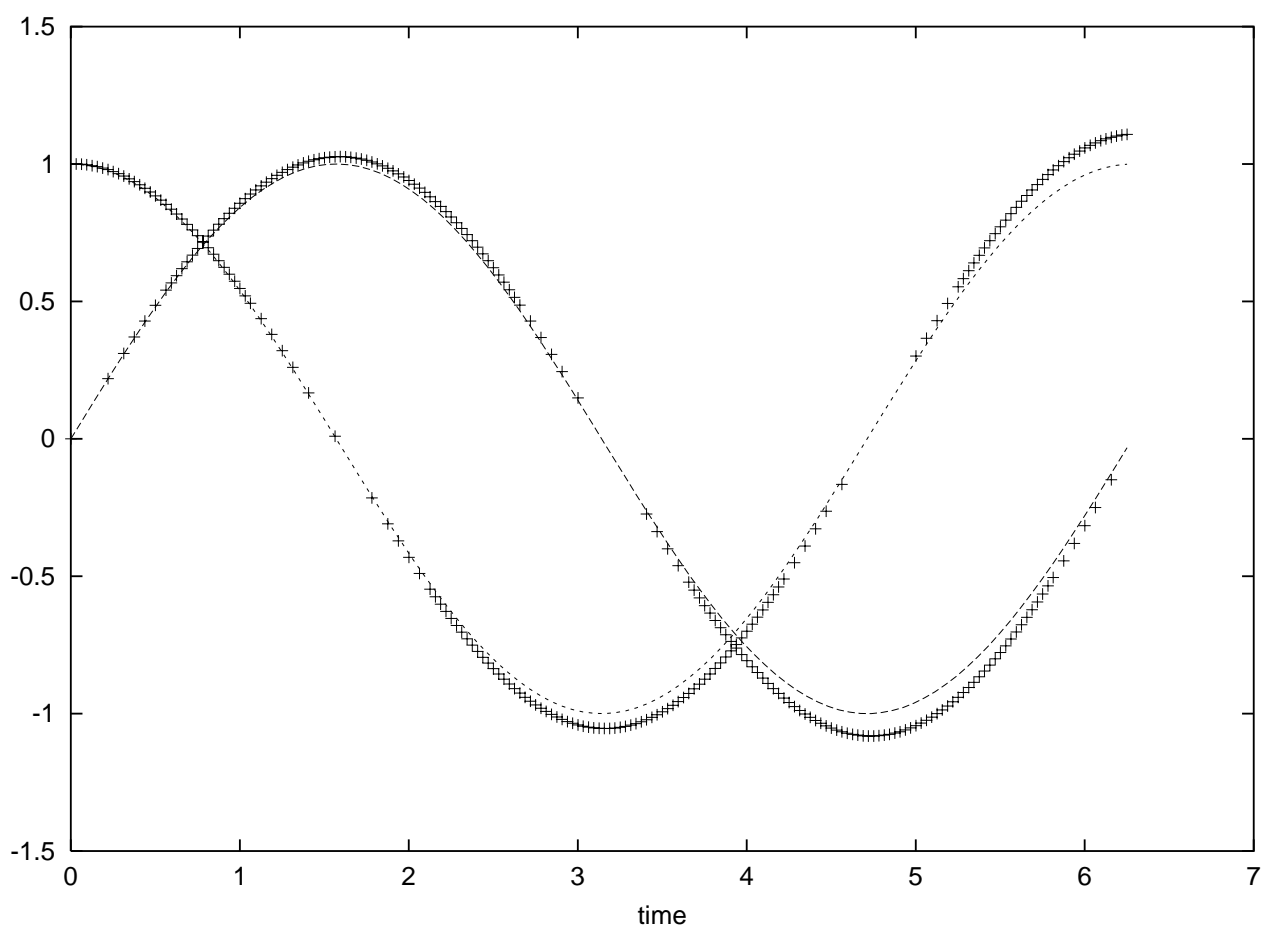




\section{Results: a Stiff System}

$$
\dot{X}=\left[\begin{array}{cc}
998 & 1998 \\
-999 & -1999
\end{array}\right] X \quad X(0)=\left[\begin{array}{l}
1 \\
0
\end{array}\right]
$$

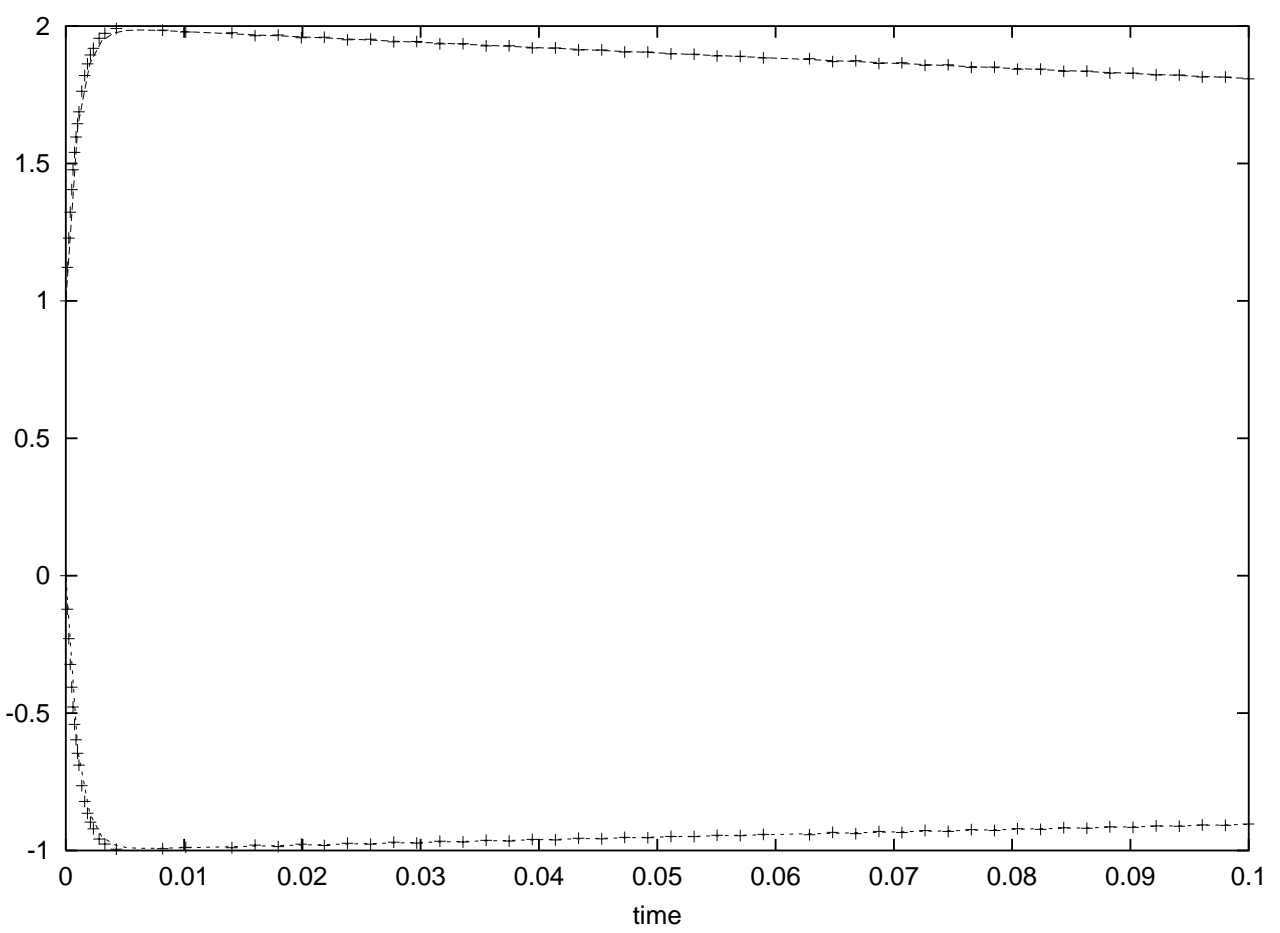




\section{Results: a Larger System-the Wine Cellar Problem}

$$
\dot{X}=\left[\begin{array}{cccccc}
0 & 1 & 0 & 0 & 0 & 0 \\
-1 & 0 & 0 & 0 & 0 & 0 \\
0 & 1 & -2 & 1 & 0 & 0 \\
0 & 0 & 1 & -2 & 1 & 0 \\
0 & 0 & 0 & 1 & -2 & 1 \\
0 & 0 & 0 & 0 & 0 & 0
\end{array}\right] X \quad X(0)=\left[\begin{array}{l}
0 \\
1 \\
0 \\
0 \\
0 \\
0
\end{array}\right]
$$

This is a simple discretization of the wine cellar problem; a sinusoidal temperature source (from the first two equations) sits on the end of a semi-infinite 1-D heat conductor. Here $\infty=4$. The large daily temperature swing on the surface is damped by the earth; only small oscillations make their way to the cellar. 


\section{Results: the Wine Cellar Problem, continued}

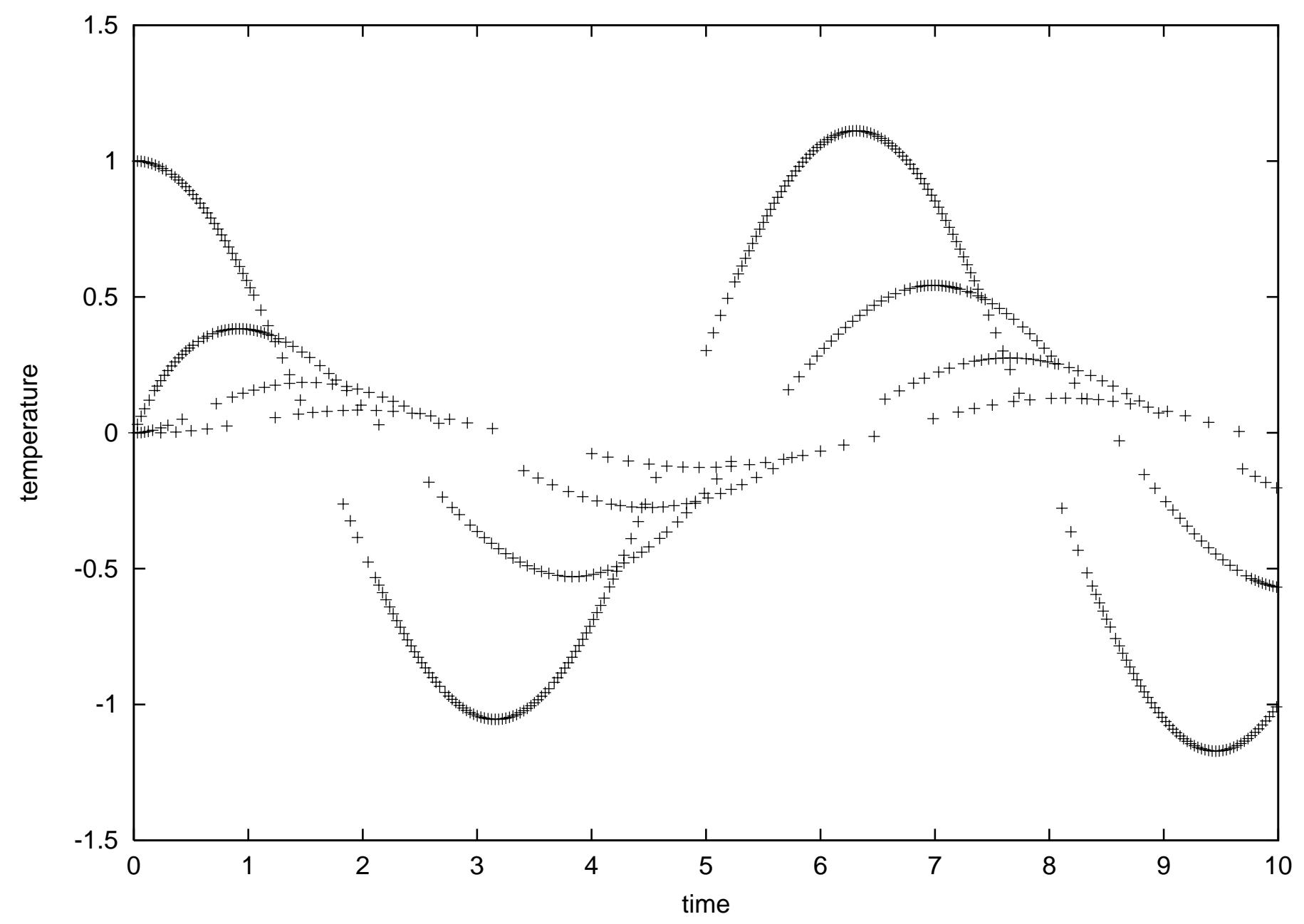




\section{Results: Van der Pol oscillator}

$$
\left[\begin{array}{l}
\dot{x} \\
\dot{y}
\end{array}\right]=\left[\begin{array}{c}
y \\
-x-\epsilon\left(x^{2}-1\right) y
\end{array}\right]
$$

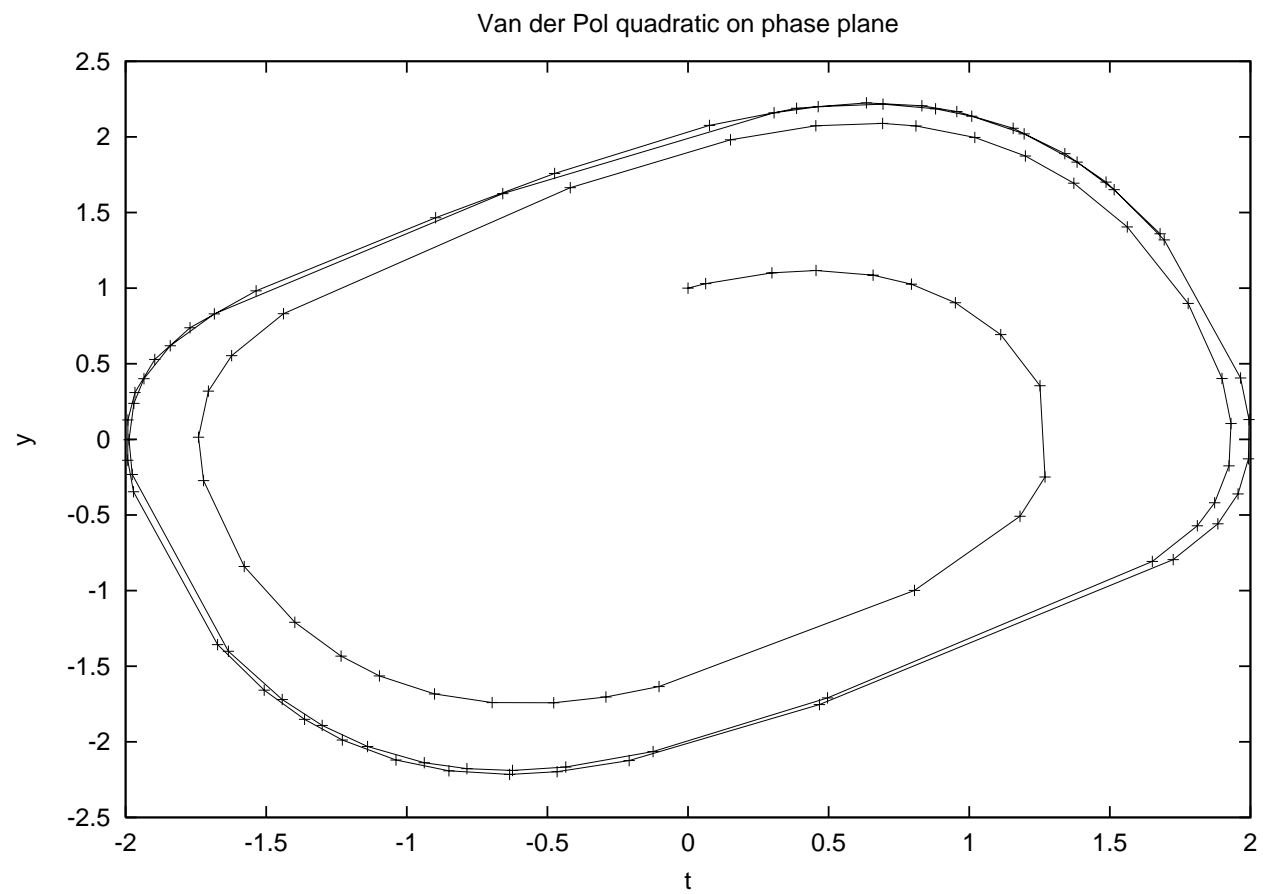




\section{Results: Periodic Convection on $x \in[0,1)$}

$$
u_{t}=u_{x} \quad u(0, t)=u(1, t)
$$

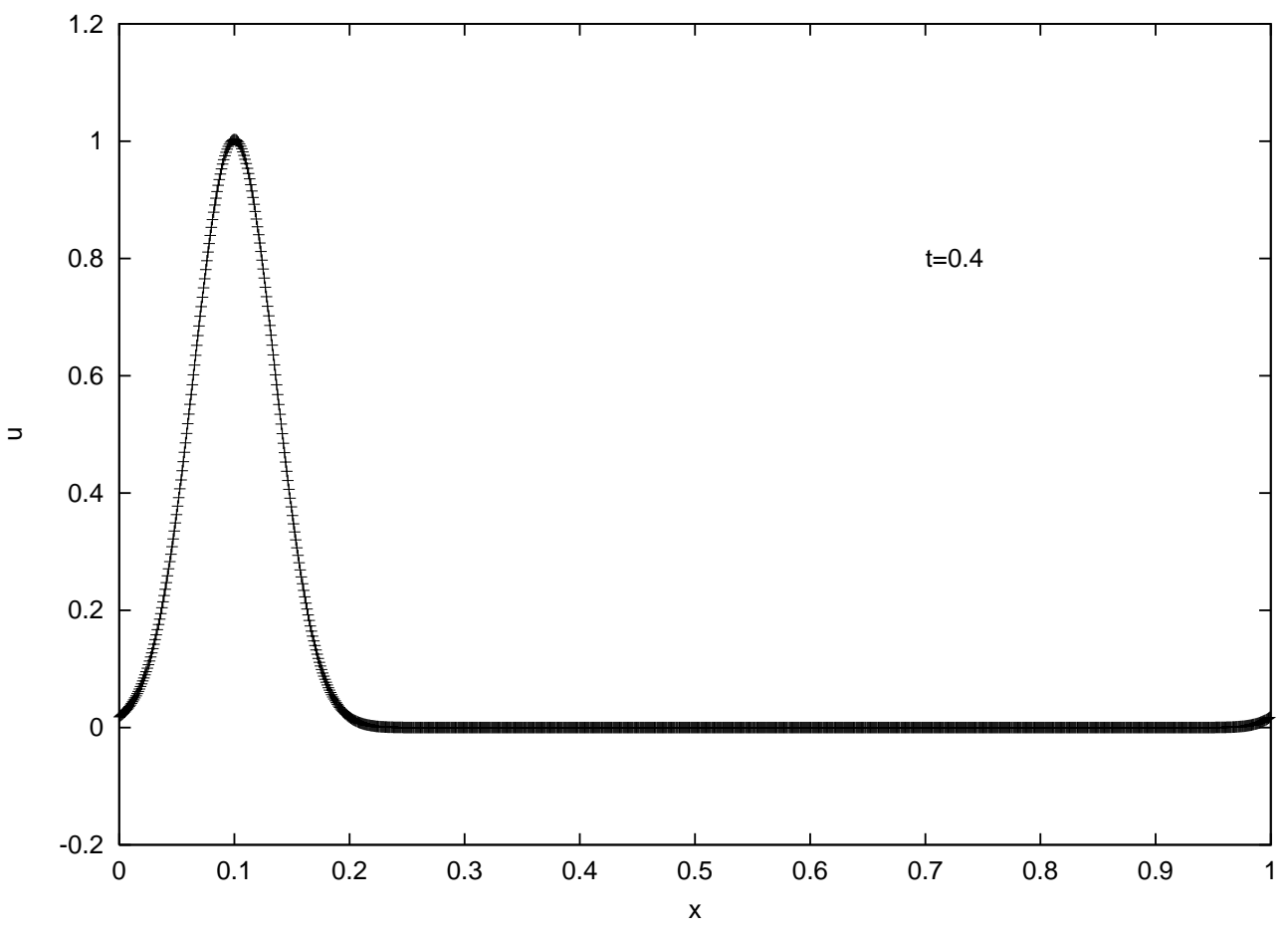




\section{All Wind and No Tunnel?}

So just how fast is it? ... not very!

- Actually runs several times slower than RK2

- Turns out small oscillations are over-compensating, causing short-time repost of every single element

- Oscillations do decrease in magnitude as $\epsilon \longrightarrow 0$, but remain present

- Suspect that improving prediction method will fix

- If we can fix it, may provide several-fold improvement 


\section{Stepping in to the Future: Our Plans}

- Fix the incessant reposting problem

- Incorporate into our hashed oct-tree vortex particle code

- Model, instead of stepping, certain small-scale motions. A different way to incorporate subgrid models?

- Multiple-pass solutions? Richardson extrapolation? 
Questions? 


\title{
LES/DES Incompressible Flow Computations/Analysis \& Development
}

\author{
Rose McCallen, Tim Dunn, Roxana Greenman, \\ Greg Laskowski, Dan Flowers, Jerry Owens \\ Lawrence Livermore National Laboratory \\ Livermore, $\mathrm{CA}$
}

March 29, 2001 


\section{The project focus is on a validated simulation capability}

Goal

To identify and develop simulation methods which can accurately predict the flow-field of heavy vehicles

Application of methods to investigate drag reduction strategies

Approach

Carefully validate the simulations with experimental data

Model

Incompressible/compressible model

Finite element method (FEM)

Large Eddy/Detached Eddy Simulation (LES/DES)

Parallel computing

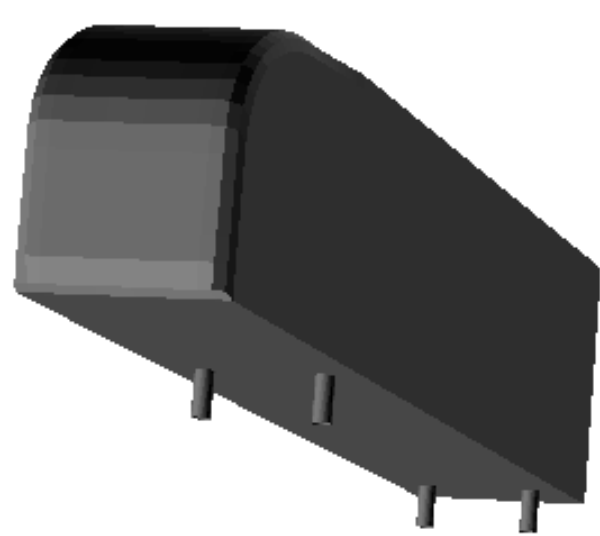




\section{In-house tools provide flexibility and access to internal resources to investigate modeling issues}

Numerical and Modeling Issues

Spatial discretization

Time integration

Turbulence modeling

Parallelization

Solvers

Meshing

Visualization

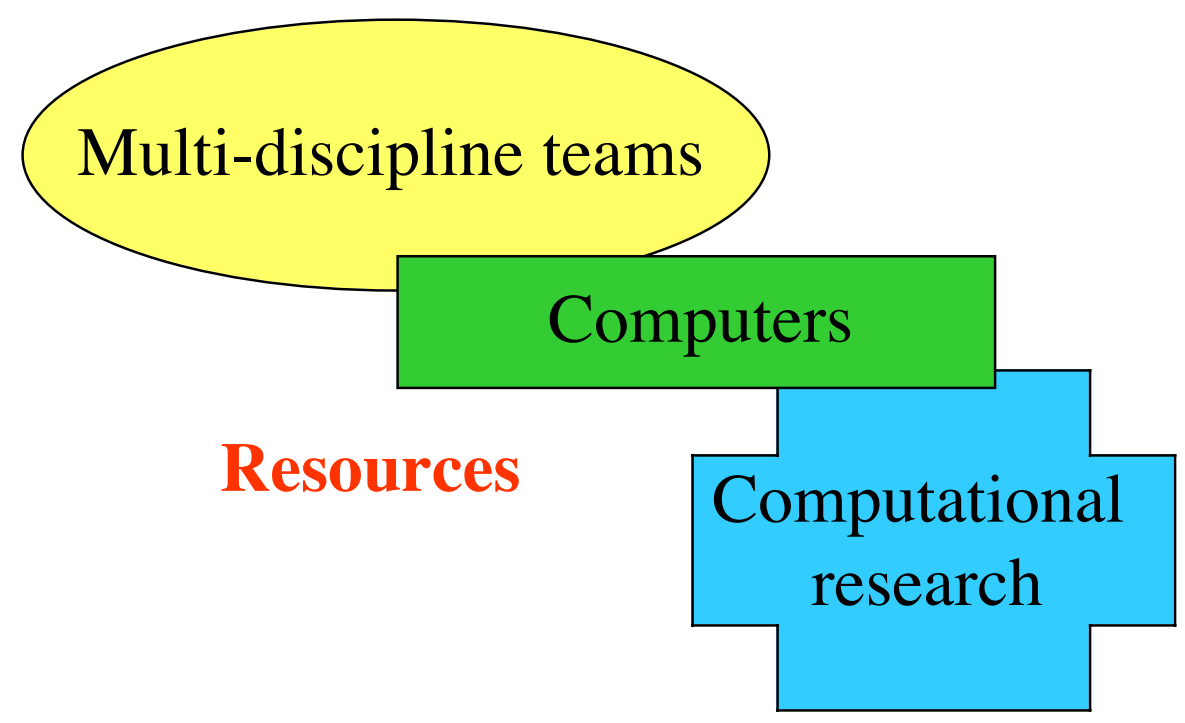

Benefits

Provides a flexible testbed

Allows the simulation of large-scale, unsteady, 3-D complex geometry

Access to advanced research and development

Disadvantage

Some options not available - implementation required 


\section{The LLNL effort is divided between Project Management and Technical efforts}

\begin{tabular}{|l|l|l|l|l|}
\hline \multicolumn{2}{|c|}{ LLNL } & Budget plan, \\
\hline \multicolumn{1}{|c|}{ FY01 Tasks } & Oct Nov Dec Jan Feb Mar Apr May Jun Jul Aug Sep & Website, etc \\
\hline & & & & Mtg at SAE \\
Conf, Group \\
Project management
\end{tabular}




\section{The LES challenges are related to physical as well as numerical modeling}

Wide range of scales

Can't resolve wall regions - problem gets too big

Grid is Reynolds number dependent

DES/LES/RANS methods - in development

Runtime very long

Evolution is over long time scales

Small time steps required

Fast/Parallel computations required - in development

Analysis

Huge data sets

Visualization required - in development

Methods for testing convergence $(\mathrm{V} \& \mathrm{~V})$ in development

Significant development being done by LLNL programs. 


\title{
Incompressible Flow Modeling and Development
}

\author{
Rose McCallen, Tim Dunn, Roxana Greenman, \\ Greg Laskowski, Dan Flowers, Jerry Owens
}


Solving the Time-Accurate, 3-D, Incompressible NavierStokes Equations

Using methods developed at LLNL

Similar formulation is used in the commercial codes FIDAP and LS-DYNA

Galerkin Finite-Element Method, Q1Q0 Element

8-node Hexahedral Brick Elements

Tri-linear Velocity

Piecewise Constant Pressure

Runs on Massively Parallel Computers

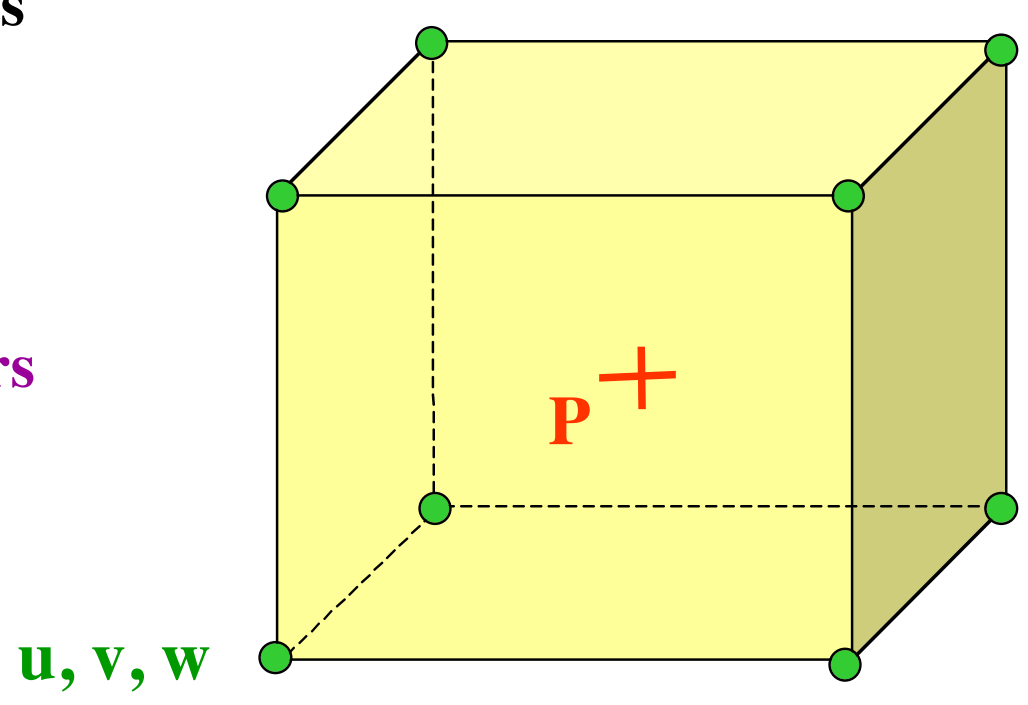


The Discretized Incompressible Momentum and Continuity Equations

Matrix Form

$$
\begin{aligned}
& M \dot{u}+(K+N(u)) u+C P=f \\
& C^{T} u=0
\end{aligned}
$$

With explicit forward Euler time integration

$$
\left[\begin{array}{cc}
M_{L} & C \\
C^{T} & 0
\end{array}\right]\left(\begin{array}{c}
v \\
P
\end{array}\right)=\left(\begin{array}{c}
F \\
0
\end{array}\right) \quad \begin{gathered}
\text { coupled system is } \\
\text { too big }
\end{gathered}
$$

The above system is NOT Positive Definite and it is more efficient to use the Schur reduced form of the matrix

$$
\begin{aligned}
& \left(C^{T} M_{L}^{-1} C\right) P=R H S \\
& u_{n+1}=u_{n}+\ldots
\end{aligned}
$$




\section{The Finite Element Interface (FEI) is used to assemble and solve the matrix}

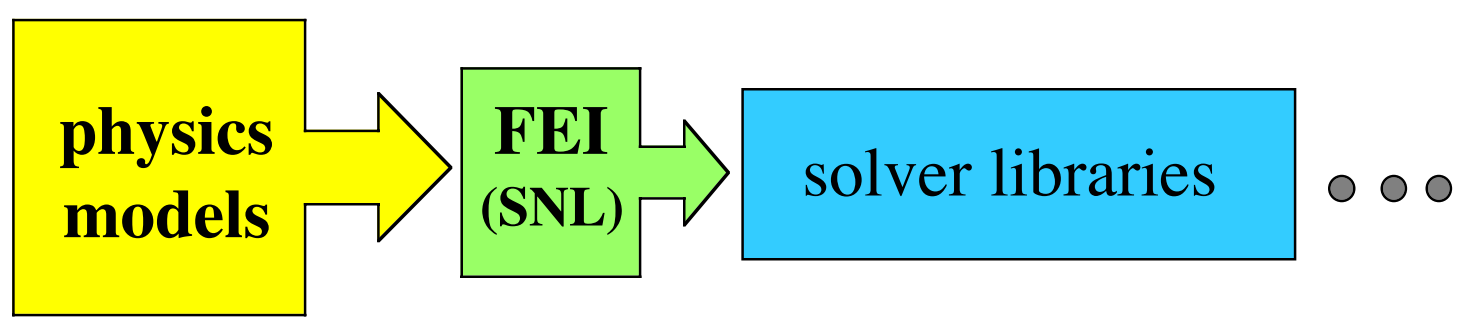

Advantages: many Krylov solvers and preconditioners become available

Advanced Solvers: HYPRE libraries developed at LLNL CASC

- Solvers

- Generalized minimal residual (GMRES)

- Conjugate gradient (CG)

- Preconditioners

- Incomplete LU

- Sparse approximate inverse

- Algebraic multigrid (AMG) 


\section{Recent code development efforts have focused on speed-up, accuracy, and turbulence modeling}

Code speedup (supported in part by internal funding)

- Reuse of matrix with FEI

- Stabilization

- Minimum residual or A-Congugate acceleration

- Implicit/Semi-Implicit (in progress)

Accuracy

- Flexibility (algorithm reformulation)

- Full spatial integration

- Higher order time integration (in progress)

Turbulence Modeling

- LES

- RANS

- DES 


\section{Pressure stabilization has been added to improve solver}

convergence

Without Stabilization

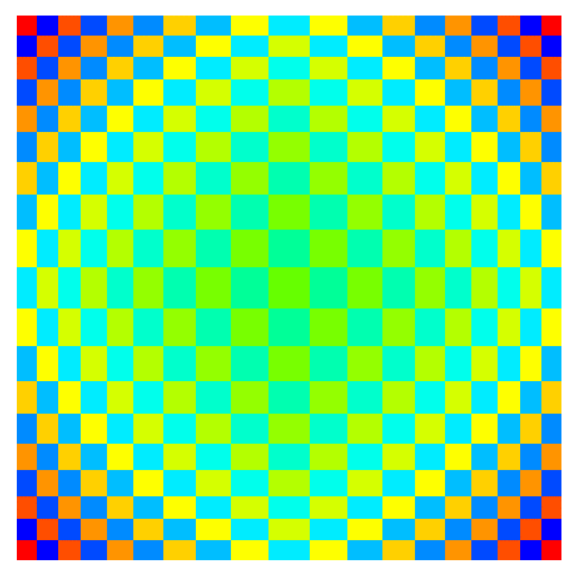

With Stabilization

Pressure

Coupling

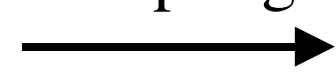

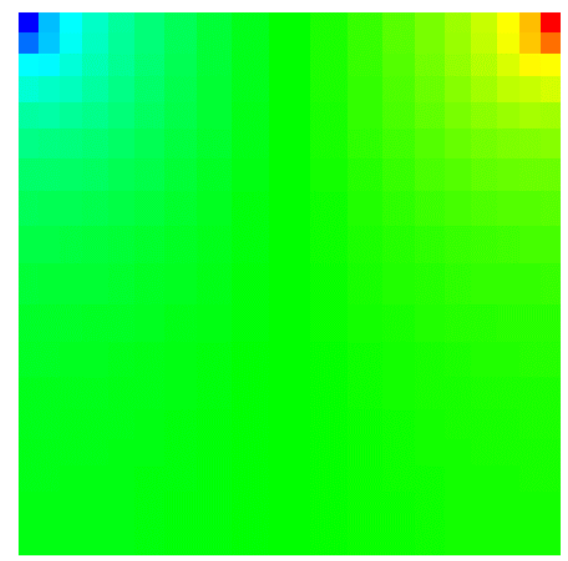

\section{Lid Driven Cavity}

- Stabilization adds coupling between neighboring elements

- Global - coupling between all elements

- Local - coupling between elements within macro-elements

- Can improve speed by factor of 2

- Reduces number of iterations

- Allows use of faster solvers (AMG) 


\section{Improving the initial guess}

Minimum Residual Projection / A-Conjugate Projection

$$
\begin{gathered}
{\left[\begin{array}{c}
x_{1}^{(-n)} \\
x_{2}^{(-n)} \\
\vdots
\end{array}\right] \cdots\left[\begin{array}{c}
x_{1}^{(-2)} \\
x_{2}^{(-2)} \\
\vdots
\end{array}\right],\left[\begin{array}{c}
x_{1}^{(-1)} \\
x_{2}^{(-1)} \\
\vdots
\end{array}\right]} \\
\text { Previous "n" } \\
\text { Solution Vectors }
\end{gathered}
$$

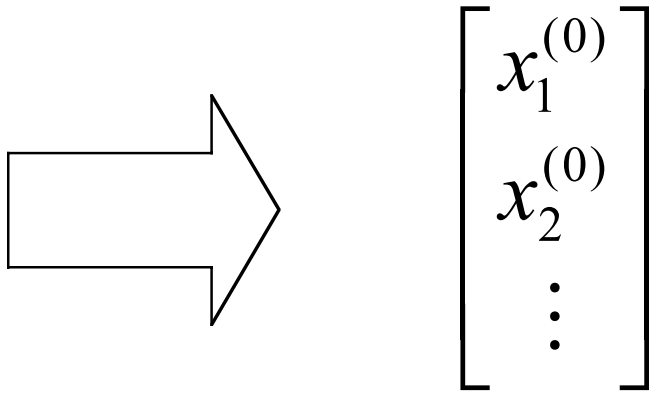

\section{Initial Guess}

Can improve speed by factor of 2 


\section{Turbulence Modeling}

DNS

Resolve all scales of turbulence

Impractical for even moderate $\mathbf{R e}$ LES

Resolve 'large' scales of turbulence

Resolve near wall region or model near wall regions

Model 'small' scales

Smagorinsky model

Dynamic model

Filtered Structure Function Models

Challenging for high Re

RANS

Model all scales of turbulence

Resolve near wall region or model near wall region

Model for 'turbulent viscosity'

Reynolds stress models

2-equation: 'k-l', 'k- $\boldsymbol{\varepsilon}$ ', $k-\omega$ '

1-equation: Baldwin-Barth, Spalart-Allmaras

Algebraic Models 


\section{How about a hybrid model?}

Choice between LES or RANS

LES

Time accurate

Sacrifice computational cost for greater accuracy

Issues concerning near wall modeling for separated flow

RANS

Time averaged

Sacrifice accuracy for faster run times

Detached Eddy Simulation (DES)

RANS near wall and LES away from wall

Maintain time accuracy while approximating some 'small' scales 


\section{Turbulence modeling status}

Current turbulence modeling capabilities

Smagorinsky model

No near-wall correction terms (i.e., van Driest damping)

Development strategy

Implement the Spalart-Allmaras model to allow for:

RANS simulations

DES simulations 


\section{Progress}

Model implemented

Currently conducting small test cases to verify implementation

Short term goal

Plane channel flow simulations

$\mathbf{R e}_{\tau}=\mathbf{2 0 0 0}$

Compare with Spalart ("An Approach to Wall Modeling in Large-Eddy

Simulations", Physics of Fluids, Vol. 12 no. 7, July 2000, pp. 1629).

Compare with VKI report 1998-21

Long term goal

Three-dimensional, unsteady truck simulations 


\title{
Incompressible Flow Computations and Analysis
}

\author{
Rose McCallen, Tim Dunn, Roxana Greenman, \\ Greg Laskowski, Dan Flowers, Jerry Owens
}

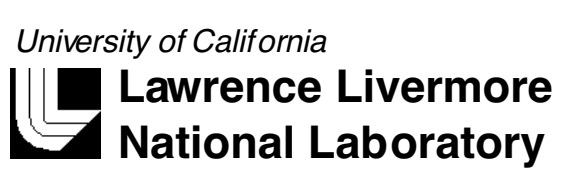

*Work performed under the auspices of the U.S. Department of Energy by Lawrence Livermore National Laboratory under Contract W-7405-ENG-48. 


\section{Verification \& validation and scaling \& timing for the incompressible model}

V\&V cases provide guidance for wind tunnel simulations

Laminar Flat Plate

Compared with theoretical Blasius solution

Speed-up and scaling results

Channel Flow

Comparison of laminar case to theory

Turbulent case (in progress)

Sphere

Comparison to experiment (in progress)

Simulations for NASA 7x10 Wind Tunnel with GTS

Determining inflow/boundary conditions

Empty tunnel calculations (in progress)

Estimating grid/runtime 


\section{Laminar incompressible flat plate -- flow and geometry description}

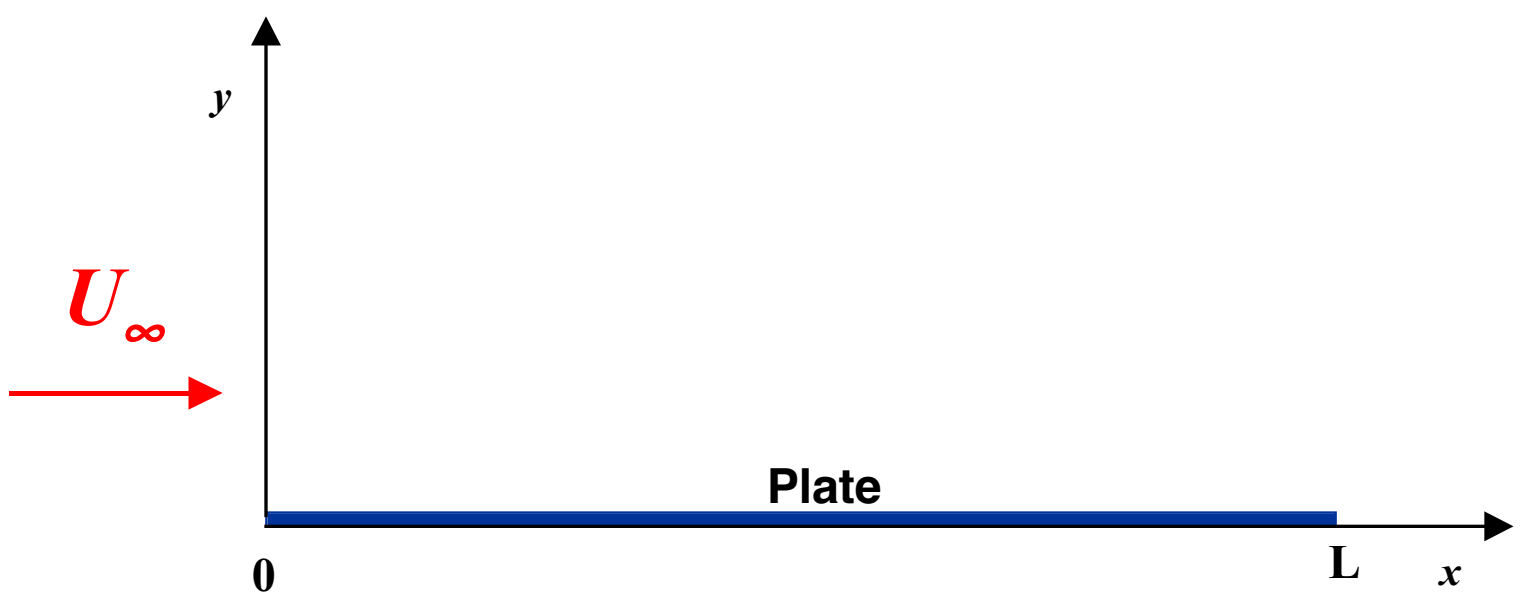

Computational domain

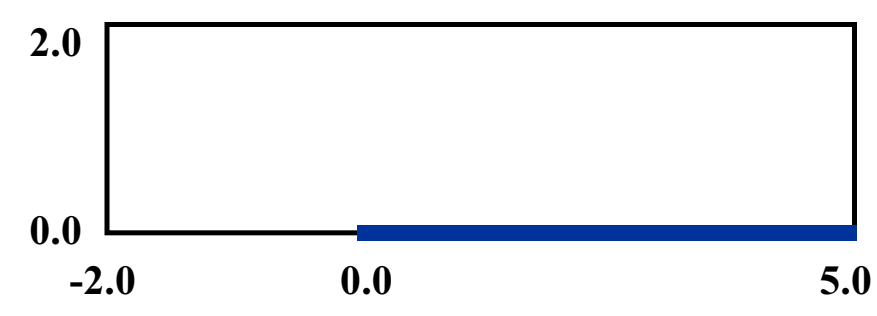

A leading-edge singularity makes this a challenging problem. 


\section{Predicted velocity compares with Blasius boundary layer theory for laminar flat plate}
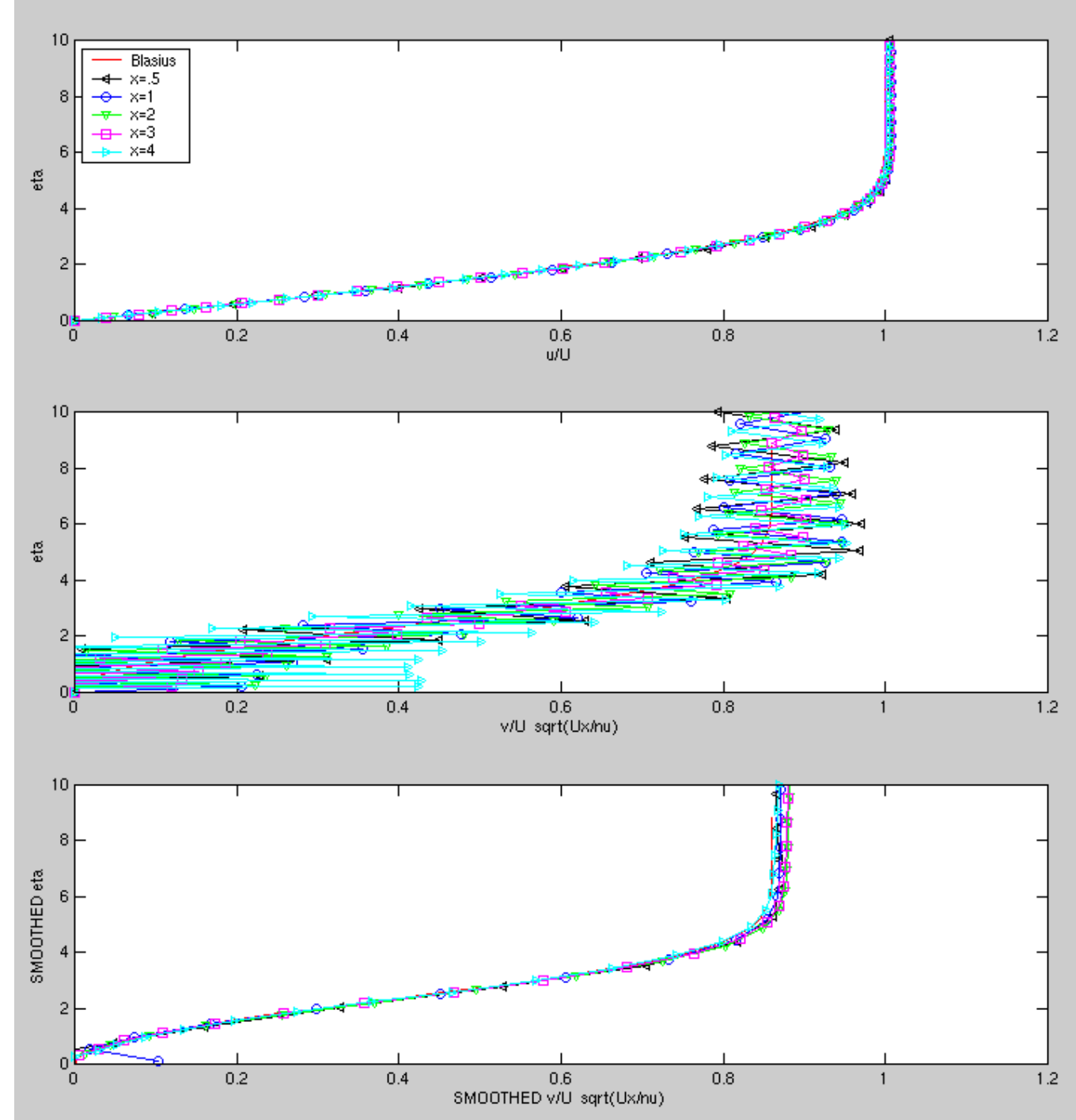

Mesh 235 x $100=$ 23,500 elements

\author{
U-velocity \\ agrees with theory
}

strict divergence free condition results in oscillatory $\mathrm{V}$-velocity

\author{
smoothed V-velocity \\ (averaged every other point) \\ agrees with theory
}




\section{Projection method can improve solution speed}

\section{Baseline}

Conjugate gradient solver

Parallel sparse approximate

inverse preconditioner

Schur reduction

No projection method

A-conjugate or minimum residual projection methods

Option to choose how many previous answers for next guess

\section{Flat Plate}

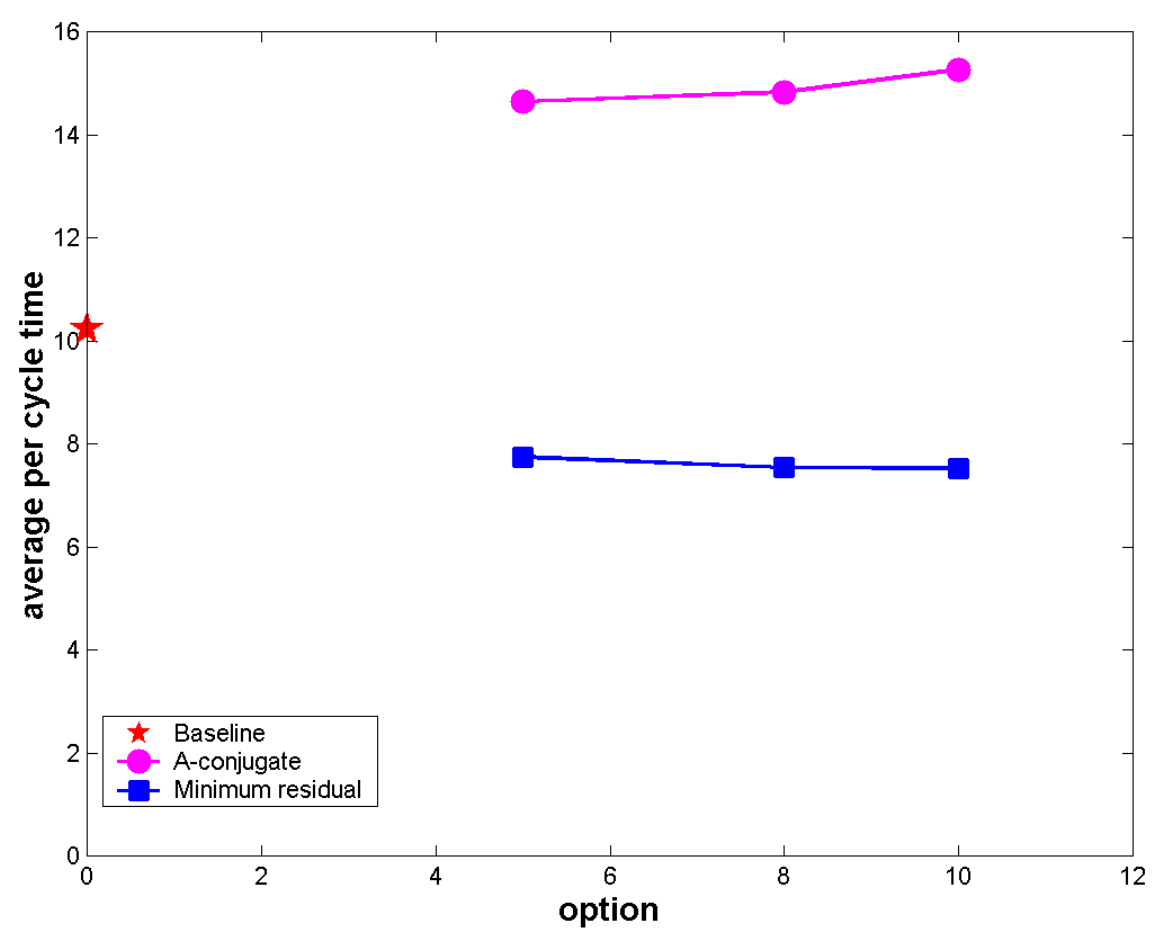




\section{Preliminary results show linear scaling}

Flat Plate

1 domain per processor

Minimum residual projection method with option 10

4 processors/node

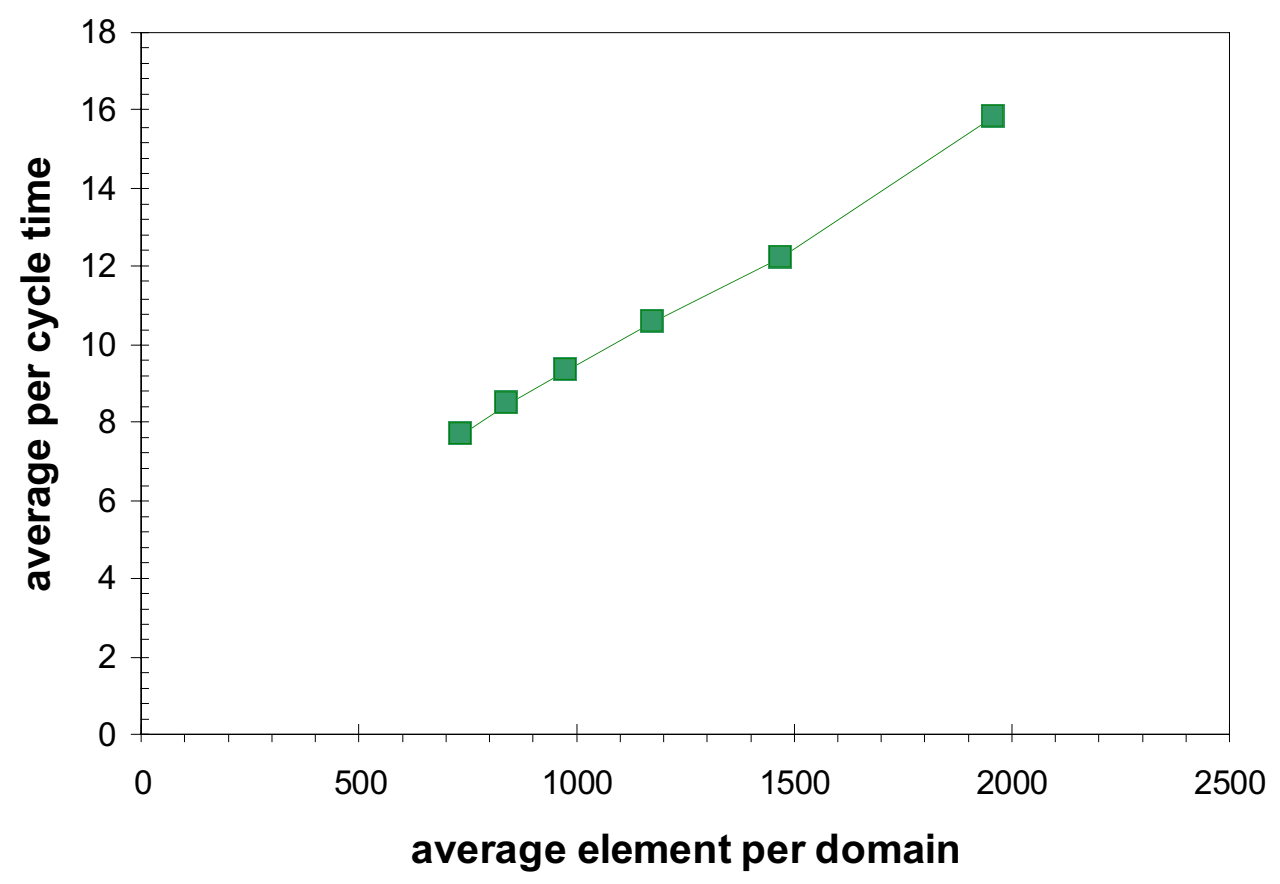

Series 1 


\section{Laminar channel flow is accurately predicted}

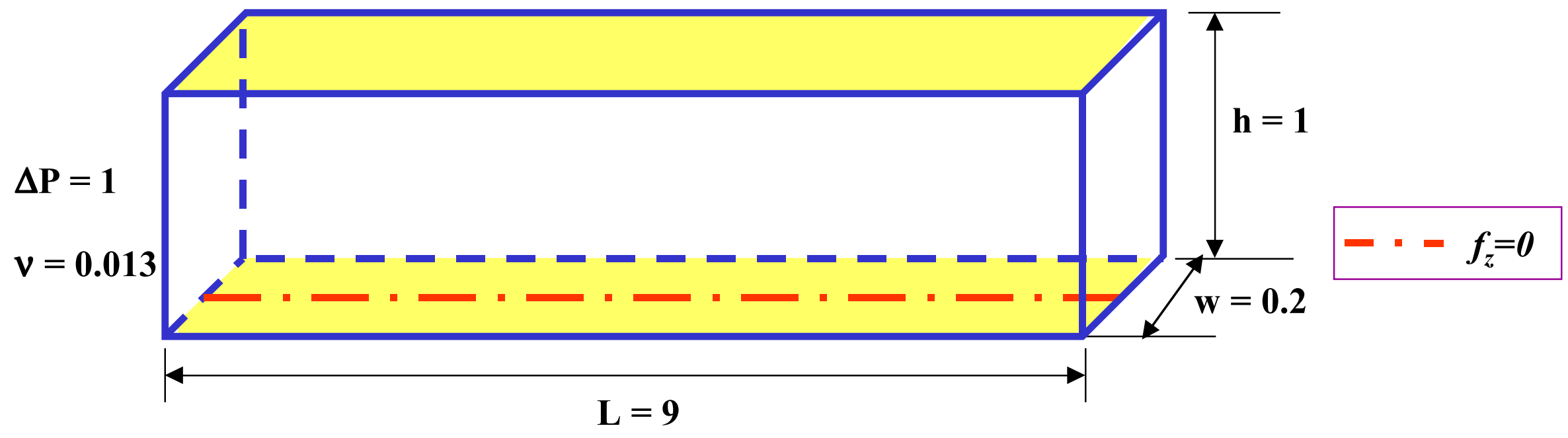

Channel Theory

Computational Results

$$
U_{\max }=-\frac{d p}{d x} \frac{h^{2}}{8 v}
$$

$$
\mathrm{P}_{\text {calc }}=0.9952, \mathrm{P}_{\text {theo }}=1.0, \text { error }=-0.48 \%
$$$$
U \max _{\text {calc }}=1.0427, U \max _{\text {theo }}=1.0417 \text {, error }=0.096 \%
$$$$
U \operatorname{avg}_{\text {calc }}=0.6934, U \operatorname{avg}_{\text {theo }}=0.6945 \text {, error }=-0.15 \%
$$ 


\section{Computational results of laminar channel flow with uniform mesh}

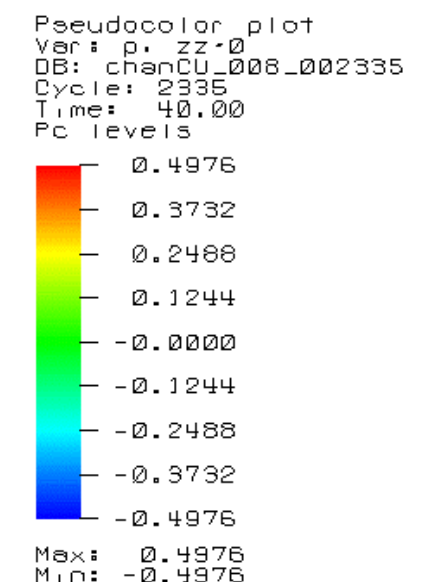

\section{PRESSURE}
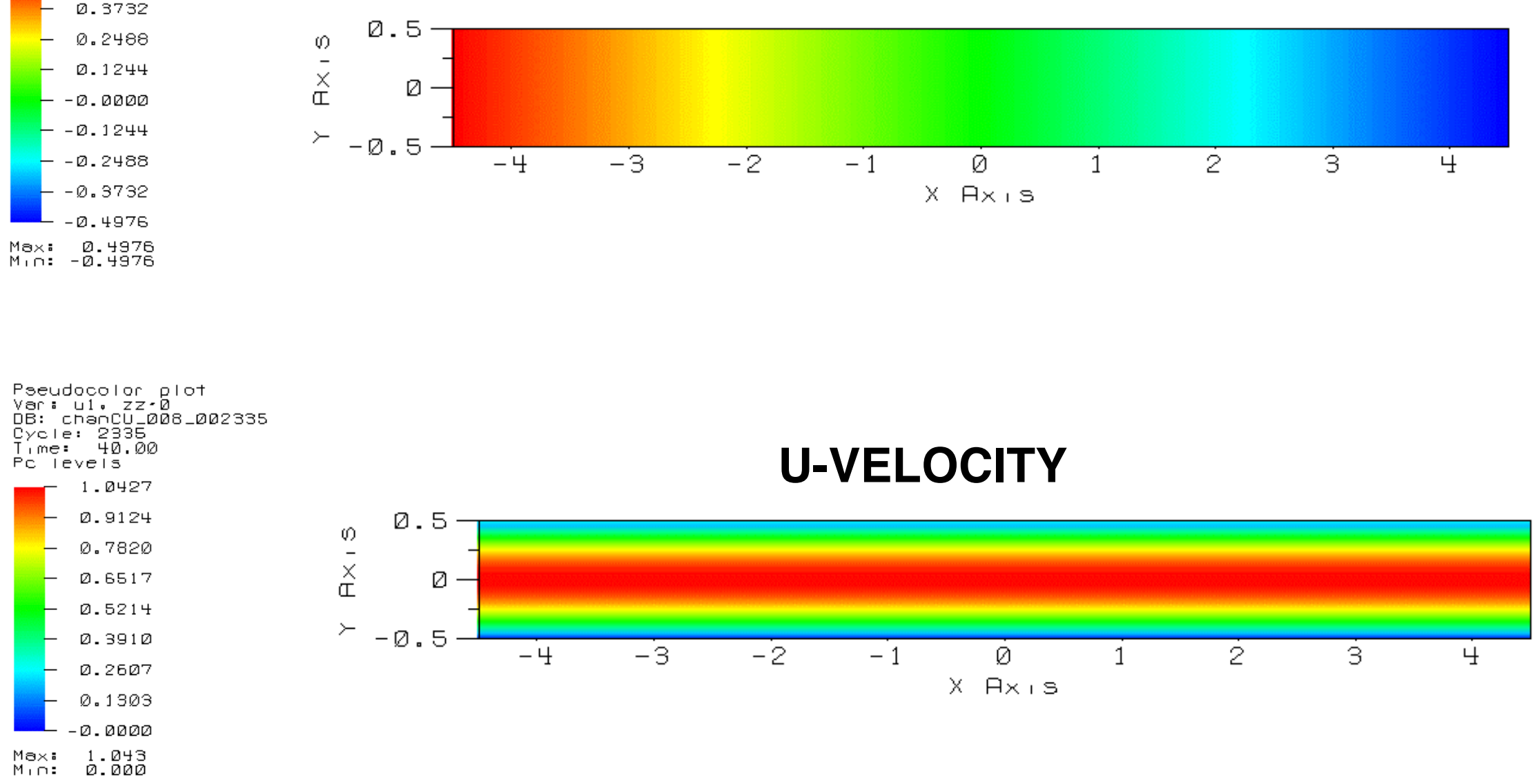

Mesh: 180 × 20 x $4=14,400$ elements 
Incompressible laminar sphere (in progress)

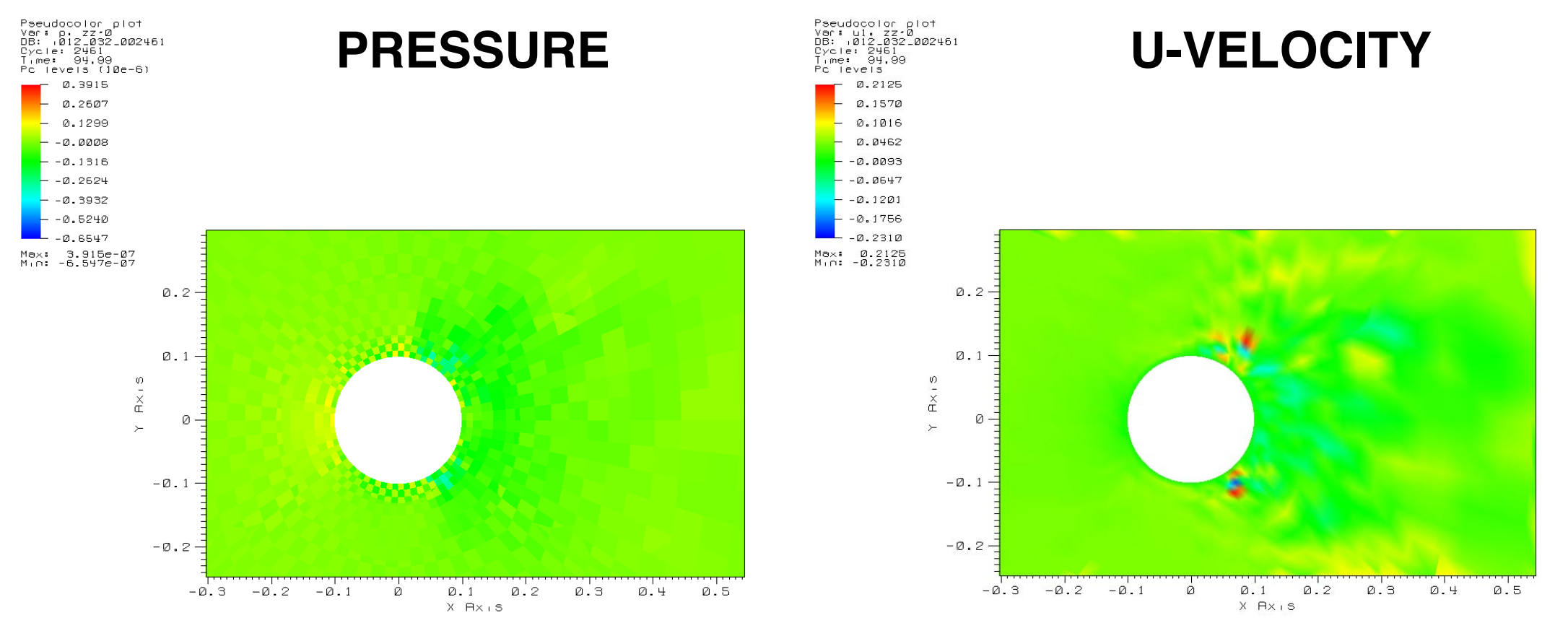




\section{Empty wind tunnel calculations to establish correct inflow and boundary conditions}

- Determine tunnel inlet length and conditions to capture correct inlet velocity profile at test section entrance

- Assumptions

- Tunnel walls are parallel and flat

- Cross-section matches test section entrance
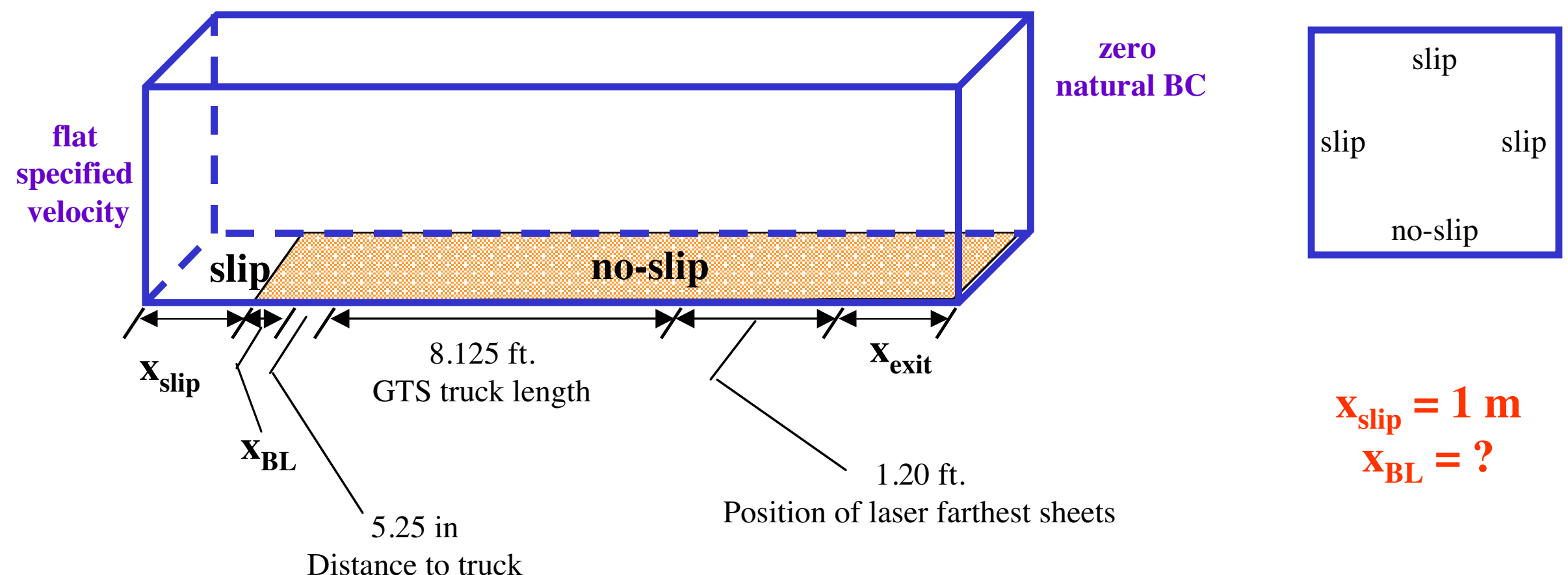

$$
\begin{gathered}
\mathrm{x}_{\text {slip }}=1 \mathrm{~m} \\
\mathrm{x}_{\mathrm{BL}}=?
\end{gathered}
$$

Distance to truck 


\section{Preliminary calculations for empty tunnel provide expected entrance length, $x_{B L} \sim 3.5 \mathrm{~m}$}

Time-averaged velocity profile

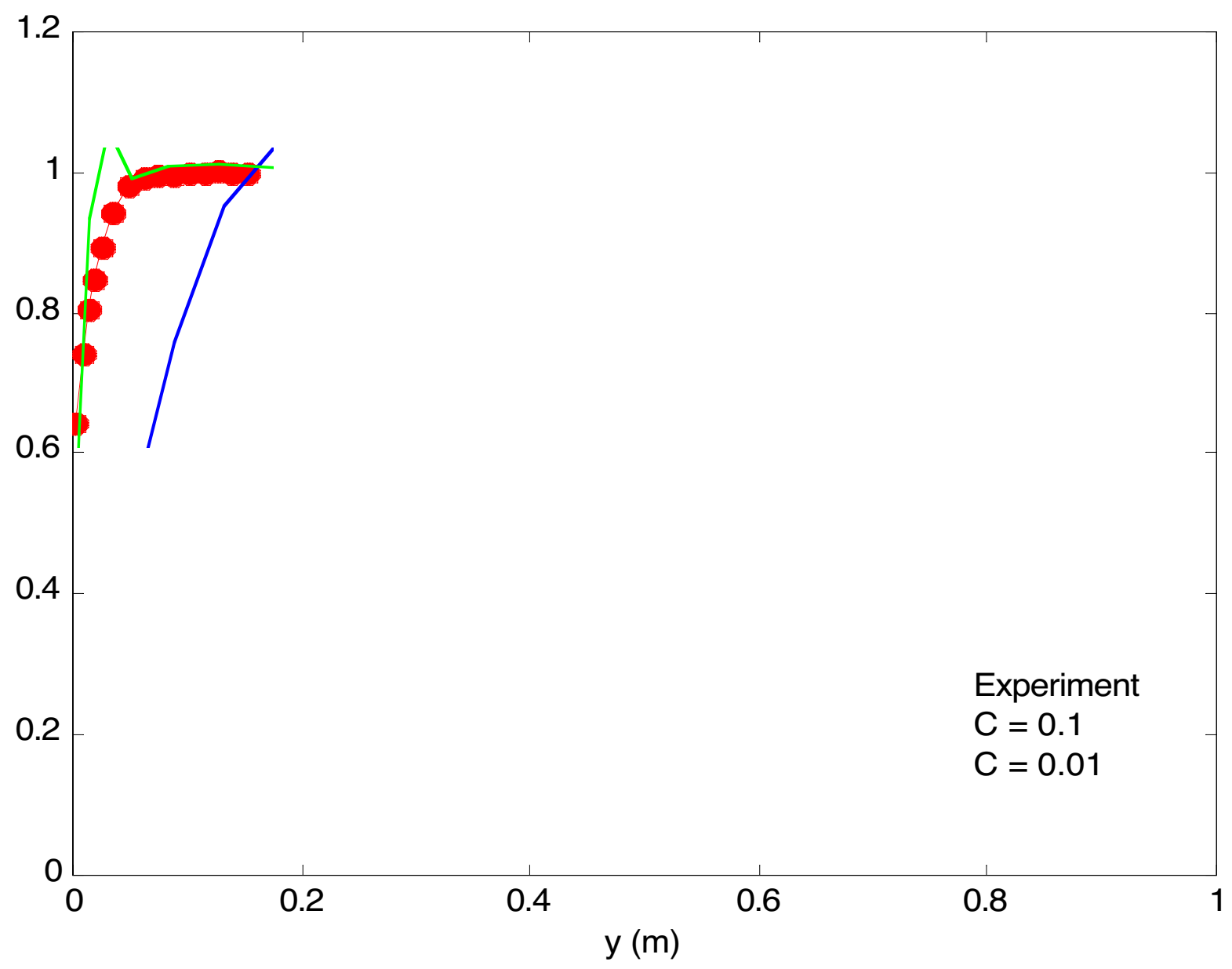




\section{Eddy viscosity is too high for stretched grids near the wall}

Stretched grids give to high an eddy viscosity

$$
\mathbf{C}_{\text {smag }}=0.1 \quad\left(\frac{v_{T}}{v}\right)_{\max } \cong 1,500
$$

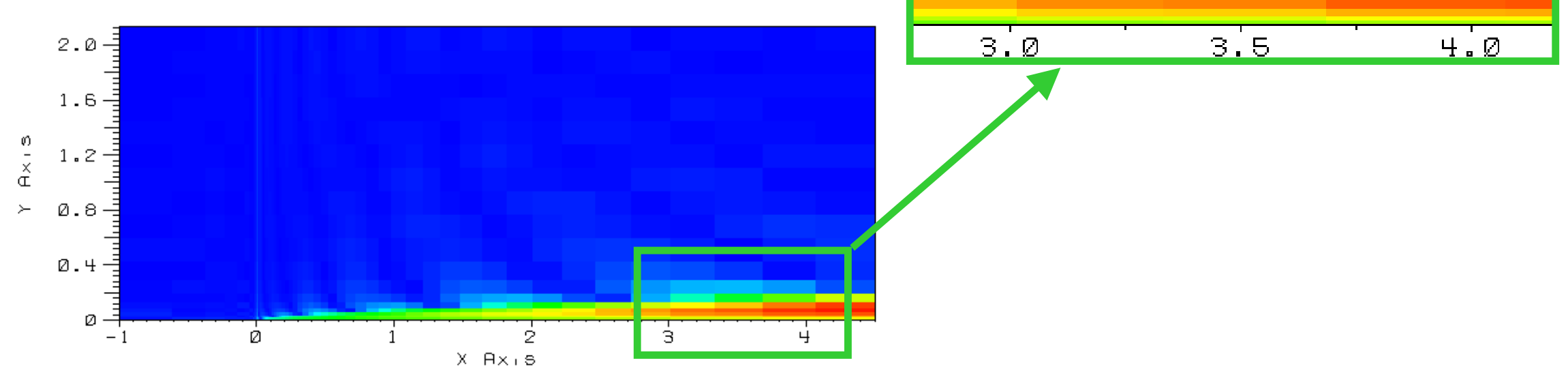

Lowering model coefficient lowers eddy viscosity

$$
\begin{aligned}
& \mathrm{C}_{\text {smag }}=0.01 \\
& \left(\frac{v_{T}}{v}\right)_{\max } \cong 50
\end{aligned}
$$

Grid refinement is essential

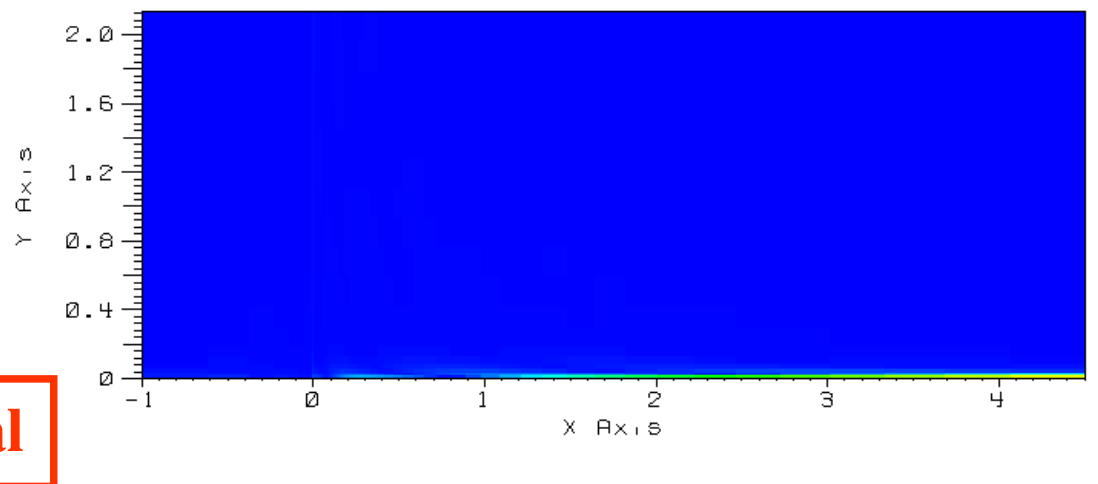




\section{Preliminary timings for empty tunnel calculations provide estimates for solutions speed requirements}

\section{Empty Tunnel Calculation}

Time step is controlled at the noslip leading edge

Current solution time with 16 processors

$\sim 10 \mathrm{sec} /$ cycle for 33,000elements

$\sim 300$ microsec/element/time step

Parallel computing is essential

Can refine stretched grid and maintain solution speed by adding processors 


\section{Evaluation of problem run times for GTS using empty tunnel results}

Assuming :

8m domain length

$\mathrm{Ma}=0.1$ or $\mathrm{U} \sim 30 \mathrm{~m} / \mathrm{s}$

Time step $\mathrm{O}\left(10^{-5}\right)$ with $\Delta=0.005 \mathrm{~m}$ - may not be reasonable

$10 \mathrm{sec} / \mathrm{cycle}$ with $2000 \mathrm{elem} / \mathrm{proc}$ - may not be reasonable

\section{Estimated problem run time :}

For fully explicit scheme $=>$ diffusion limited time step $O\left(10^{-5}\right) \Rightarrow 12$ days

May need factor of $\mathbf{1 0}$ speed-up in solution time.

Semi-implicit schemes are being considered.

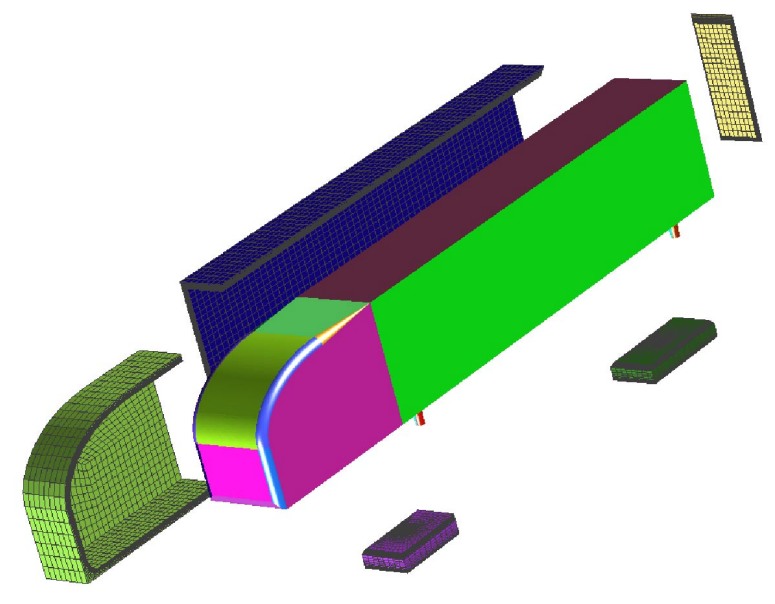




\section{Suggested alternate plan, if GTS requirements exceed solution speed and resources}

Focus on base pressure predictions

Area in need of advanced space/time-accurate modeling

Reduced problem size

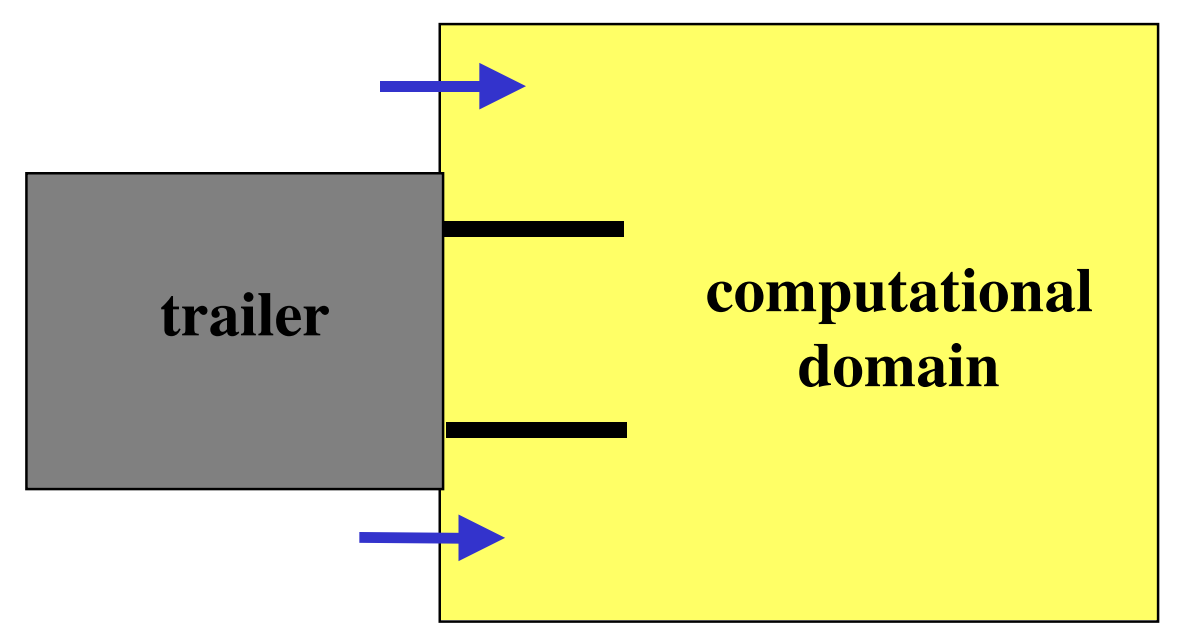




\section{Visualization}

\section{Rose McCallen, Tim Dunn, Roxana Greenman, Greg Laskowski, Dan Flowers, Jerry Owens}


New transient analysis capability

Transparent (see through) contour plots

Alpha composite of $\mathbf{3}$ movies

Moving plane with transparent contours

Movies 